\title{
Cognitive and biological individual differences in problematic alcohol use
}

Citation for published version (APA):

van den Wildenberg, E. (2007). Cognitive and biological individual differences in problematic alcohol use. [Doctoral Thesis, Maastricht University]. Datawyse / Universitaire Pers Maastricht. https://doi.org/10.26481/dis.20070614ew

Document status and date:

Published: 01/01/2007

DOI:

10.26481/dis.20070614ew

Document Version:

Publisher's PDF, also known as Version of record

\section{Please check the document version of this publication:}

- A submitted manuscript is the version of the article upon submission and before peer-review. There can be important differences between the submitted version and the official published version of record.

People interested in the research are advised to contact the author for the final version of the publication, or visit the DOI to the publisher's website.

- The final author version and the galley proof are versions of the publication after peer review.

- The final published version features the final layout of the paper including the volume, issue and page numbers.

Link to publication

\footnotetext{
General rights rights.

- You may freely distribute the URL identifying the publication in the public portal. please follow below link for the End User Agreement:

www.umlib.nl/taverne-license

Take down policy

If you believe that this document breaches copyright please contact us at:

repository@maastrichtuniversity.nl

providing details and we will investigate your claim.
}

Copyright and moral rights for the publications made accessible in the public portal are retained by the authors and/or other copyright owners and it is a condition of accessing publications that users recognise and abide by the legal requirements associated with these

- Users may download and print one copy of any publication from the public portal for the purpose of private study or research.

- You may not further distribute the material or use it for any profit-making activity or commercial gain

If the publication is distributed under the terms of Article $25 \mathrm{fa}$ of the Dutch Copyright Act, indicated by the "Taverne" license above, 
Cognitive and Biological Individual Differences in Problematic Alcohol Use 
Colophon

Cover design: Björn Nelissen

Production: Datawyse, Universitaire Pers Maastricht

(C) Esther van den Wildenberg, Maastricht 2007

ISBN 978-90-5278-634-6

Part of the studies presented in this dissertation was funded by ZonMw grant 310-00-065 (Programma Verslaving) 


\title{
Cognitive and Biological Individual Differences in Problematic Alcohol Use
}

\author{
PROEFSCHRIFT \\ ter verkrijging van de graad van doctor aan de Universiteit Maastricht, \\ op gezag van de Rector Magnificus, prof. mr. G.P.M.F. Mols, \\ volgens het besluit van het College van Decanen, \\ in het openbaar te verdedigen \\ op donderdag 14 juni 2007 om 14.00 uur
}

door

Esther van den Wildenberg

geboren te Oosterhout

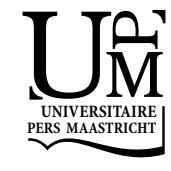




\section{Promotores}

Prof. dr. R.W. Wiers

Prof. dr. A.T.M. Jansen

\section{Beoordelingscommissie}

Prof. dr. R.A. Knibbe (voorzitter)

Prof. dr. W. van den Brink (Academisch Medisch Centrum, Amsterdam)

Prof. dr. J.J. van Os

Prof. dr. M.L. Peters

Prof. dr. A.N.M. Schoffelmeer (VU Medisch Centrum, Amsterdam) 


\section{CONTENTS}

Chapter 1 General Introduction $\quad 7$

Chapter 2 Is the strength of implicit alcohol associations correlated with alcohol-induced heart-rate acceleration?

Chapter 3 Do implicit alcohol arousal and approach associations correlate with attentional bias in alcohol-dependent patients?

Chapter 4 Predicting treatment response of alcohol-dependent patients receiving naltrexone with implicit and explicit alcohol-related cognitions

Chapter 5 A functional polymorphism of the mu-opioid receptor gene (OPRM1) influences cue-induced craving for alcohol in male heavy drinkers

Chapter 6 Polymorphisms of the dopamine D4 receptor gene (DRD4 VNTR) and cannabinoid CB1 receptor gene (CNRT) are not strongly related to cue-reactivity after alcohol exposure

$\begin{array}{ll}\text { Chapter } 7 \text { General Discussion } & 147\end{array}$

$\begin{array}{ll}\text { References } & 160\end{array}$

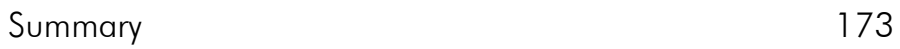

$\begin{array}{ll}\text { Samenvatting } & 179\end{array}$

Dankwoord 188

$\begin{array}{ll}\text { Curriculum Vitae } & 191\end{array}$

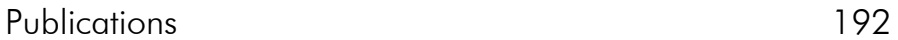



CHAPTER 1

GENERAL INTRODUCTION 


\section{INTRODUCTION}

The first chapter of this thesis provides a general background on several cognitive and biological factors that can predispose an individual to develop alcohol-related problems. First, I give a brief description of the problem alcoholism. Next, several biological and cognitive factors that might add to alcoholism liability are explored and finally the research questions and outline of this thesis will be presented.

Alcohol abuse and dependence are among the most prevalent psychiatric disorders. In 1996, the Netherlands Mental Health Survey and Incidence Study (NEMESIS; Biil et al., 1998) determined the prevalence of several psychiatric disorders in the Dutch population, based on the DSM-III-R criteria. A lifetime prevalence of $11.7 \%$ was found for alcohol abuse (with $19.3 \%$ for men and $3.9 \%$ for women) and a lifetime prevalence of $5.5 \%$ for alcohol dependence $(9 \%$ for men, $1.9 \%$ for women). The 12-month prevalence of alcohol abuse and dependence was $4.6 \%$ and $3.7 \%$ respectively, percentages very similar to those found in the large $\mathrm{Na}$ tional Epidemiologic Survey on Alcohol and Related Conditions (NESARC) conducted in the United States in 2001-2002 (Grant et al., 2004), which was based on the DSM-IV. Young men are overrepresented regarding the diagnosis of alcohol-related disorders.

According to the DSM-IV (American Psychiatric Association, 1994), a person suffers from alcohol abuse when a maladaptive pattern of alcohol use causes significant restrictions or suffering as becomes apparent from at least one of the following criteria: 1) a failure to succeed in fulfilling obligations related to work, school or home, 2) recurrent use in physically hazardous situations, 3) recurrent conflicts with the law, or 4) continued use despite recurrent social or interpersonal problems. Alcohol dependence is characterized by three or more of the following: 1) tolerance, 2) withdrawal, 3) more or longer use than intended, 4) persistent wish or few successful attempts to cut down drinking, 5) a large amount of time is spent on obtaining, using or recovering from the drug, 6) important social or work related activities are given up in order to use, 7) continued use despite persistent physical or psychological problems. There has been debate on the definition of what constitutes an addiction and how it should be diagnosed (e.g. de Bruijn et al., 2006; Schuckit et al., 2003; Wiers, 2004; Addiction, Supplement September 2006). Since the DSM III-R, the physiological components tolerance and withdrawal were removed as obligatory criteria for a diagnosis of dependence. This makes it difficult to make the distinction between a bad habit in which a substance is involved and an addiction (Wiers, 2004). A large prospective study showed that presence of a physiological component of dependence (tolerance and especially 
withdrawal) was related to more severe alcohol use and problems at baseline and a more severe clinical course over 5 years (Schuckit et al., 2003), emphasizing the importance of a physiological component in the diagnosis of alcohol or substance dependence. The DSM-IV also leaves no room for 'addictions' such as pathological gambling or excessive internet use, since no psycho-active substance is involved. However, since a diagnosis of dependence can, according to the DSM-IV, also be based on behavioral criteria only (and not physiological criteria per se), the necessity for addiction to be related to a psychoactive substance disappears (Wiers, 2004). Also, craving, the irresistible urge to use the substance, is at present no criterion for abuse or dependence, while it has been shown to add to the diagnostic stability (De Bruijn et al., 2006). The chronic loss of control over drinking which is characteristic to alcohol use disorders can lead to various problems such as: memory loss, increased risk of traffic accidents, increased risk of having unsafe sex, depression, thiamine deficiency (which can result in Korsakoff's syndrome) liver, pancreas, heart and neurological diseases and eventually death. Individual differences exist in the vulnerability for developing an alcohol problem. Several biological as well as cognitive factors have been identified that seem important in the development of alcohol-related disorders. Some of these factors, which are relevant to this thesis, will be described below.

\section{BIOLOGICAL FACTORS}

Different biological factors can have an influence on how a person responds to alcohol and other drugs. Here, distinction will be made between physiological responses and genetic aspects, which are, obviously, also related to each other.

\section{Physiological responses}

Individuals with a positive family history of alcoholism (FHP), are at an increased risk of developing alcohol dependence compared with individuals with a negative family history (FHN; McGue, 1999). This is partly due to their differential physiological response to alcohol. Several studies have found evidence for an attentuated response in FHPs: they report less subjective feelings of intoxication ( $\mathrm{O}^{\prime}$ Malley \& Maisto, 1985), display lower cortisol (Schuckit et al., 1987a) and prolactine reactions (Schuckit et al., 1987b), and less body sway (Schuckit, 1985). Thus, they seem less sensitive to the effects of alcohol. Other studies suggest that FHPs show a stronger response to alcohol, as becomes apparent from a stronger alcoholinduced heart rate increase (Conrod et al., 1997) and a stronger release of betaendorphin upon alcohol consumption (Gianoulakis et al., 1996a, 1996b). In men 
with a multi-generational $\mathrm{FH}$ the alcohol-induced heart rate increase was even strongly correlated with beta-endorphin release (Peterson et al., 1996).

Newlin and Thomson (1990) have integrated these seemingly paradoxical findings of attenuated and enhanced responding in FHPs in their differentiator model. This model assumes that FHPs and FHNs respond differently to alcohol both during the ascending and during the descending limb of the blood alcohol curve. During the ascending limb, FHPs show faster sensitization and during the descending limb, they show a faster tolerance. Thus, on the one hand, the immediate, stimulating, rewarding effects of alcohol that are experienced by FHPs during the ascending limb, will become stronger (sensitized) after repeated alcohol intake (e.g. reflected by enhanced psychomotor stimulation). On the other hand, they are more tolerant to the late, negative, sedative effects usually experienced during the descending limb (e.g. worse evaluation of their subjective intoxication). Due to this innate tolerance for the late, sedating effects of alcohol, the individual does not experience negative consequences of his or her drinking behavior. This can result in the absence of feedback mechanisms that normally inhibit overconsumption, thereby increasing the chance of an alcohol-related disorder (Schuckit, 1994). Contrary to what is commonly thought, having a low level of reaction is a strong predictor of a future alcohol problem (Schuckit \& Smith, 1996).

\section{Incentive sensitization}

In line with the differentiator model by Newlin and Thomson (1990), Robinson and Berridge $(1993 ; 2001$; 2003) have proposed an influential theory for the development of addictions in general: Incentive Sensitization Theory. Their focus is on the increasingly rewarding and stimulating effects of psycho-active substances directly after administration (ascending limb). The theory tries to explain why the use of addictive substances leads to compulsive use in some individuals, but not in others.

The theory consists of four main assumptions. First, addictive substances produce structural changes or neuro-adaptations in the brain (on the molecular, cellular and neural system level). Second, the brain areas that change are part of the mesocorticolimbic dopamine system, which is usually involved in natural motivation and reward (e.g. evolutionary important processes that yield a survival benefit, such as searching for food, water and partners). Third, the drug-induced neuroadaptations render the reward system hypersensitive for drugs and drug-related stimuli. Thus, the effect of the drug becomes stronger after repeated use. Fourth, the authors link two psychological processes to the development of neural sensitization. They suggest it is the "wanting" component that sensitizes, and not the hedonic, euphoric component of drug use, called "liking". As a consequence, drugs 
and drug-related stimuli obtain an incentive salience and attention is automatically drawn to them (attentional bias). The reward system can be activated without conscious awareness which can give the addict a feeling of compulsive drug seeking behavior or a strong craving for the drug, although the subjective component or "liking" has diminished. According to Everitt and Robins (2005) three phases can be distinguished in a developing addiction: voluntary actions, habitual use and compulsive use. They hypothesize that these transitions are caused by a transition from prefrontal to more striatal control over responding. Additionally, as neural sensitization has developed for one drug, often cross-sensitization occurs for other drugs and for stress. Mainly cross-sensitization for stress can increase chances of relapse since stress increases the sensitivity to drugs and vice versa (Piazza et al., 1991).

\section{Genetics}

As described above, FHPs respond differently to the effects of alcohol than FHNs. They run a higher risk of developing alcohol abuse or dependence due to genetic factors which are inherited. Research with twin and adoption studies has indicated that $50-60 \%$ of the variance in the vulnerability for alcohol dependence is associated with genetic factors (McGue, 1999). The heritability of other addictive disorders ranges from .39 for hallucinogens (also among the least addictive drugs) to approximately .70 for opiates and cocaine (also among the most addictive drugs; D. Goldman et al., 2005). Obviously, complex and heterogeneous disorders such as alcohol or substance dependence are polygenetic. Genes can have an influence on a variety of processes such as alcohol metabolism, preference, tolerance and sensitivity. The final phenotype results from interactions between these multiple genes and environmental factors. Cloninger (1987) describes two types of alcoholism which are differentially influenced by genes and environment. Type I alcoholism is characterized by a relatively late age of onset, affects both men and women, who usually display relatively low levels of antisocial behavior. This type of alcoholism is more affected by environmental influences such as divorce and less by genetic factors (less than 40\%, McGue, 1999). Type II alcoholism is characterized by a relatively early age of onset, affects mainly men, who generally show relatively high levels of antisocial behavior. Heritabilities as high as $90 \%$ have been reported for this type of alcoholism (McGue, 1999). There exist drug-specific and more general genetic factors that influence alcohol and substance dependence liability (Goldman \& Bergen, 1998). An example of a drug-specific gene is the ALDH2 gene of which the inactive variant prevents alcohol from being metabolized in the liver. The unpleasant flushing reaction which follows serves as a protective factor against alcoholism, as seen in Japanese. A more general genetic factor is for ex- 
ample the serotonin transporter gene, which has been associated with anxietyrelated personality traits (Schinka et al., 2004). Genetic variations in the dopamine (DRD2, DRD4), opioid (OPRM1), GABA (GABRA6, GABRB2) and cannabinoid (CNR1) systems have been associated with alcoholism liability as well.

How can we identify the multiple genes that influence the risk for alcoholism? Broadly, three strategies exist: association studies, linkage analysis and animal models. In an association study, cases (those having the disorder) are compared with ethnically matched controls (who do not have the disorder) with respect to the frequency of an allele of a particular candidate gene which is expected to be related to the disorder. In linkage analysis, within-family associations are identified between so-called genetic markers and the phenotype of interest. A genetic marker is a DNA sequence with an identifiable location on the chromosome which inheritance pattern can be tracked throughout the family. It signals the near location of a disease susceptibility locus, since parts of the chromosome which are closely together tend to be cotransmitted. Finally, animal models offer the opportunity to demonstrate the existence of heritable effects on behavioral phenotypes by wellcontrolled breeding patterns and rearing circumstances. Transgene and knockout mice can be studied to investigate altered gene expression after DNA manipulation.

Often, association studies fail to replicate previous findings of a relationship between a specific allele of a candidate gene and the phenotype of interest, such as alcohol or substance dependence (e.g. Arias et al., 2006). This is not surprising since a complex disorder such as alcohol dependence is very heterogenic and influenced by many genes. Gottesman and Gould (2003) re-introduced the concept of 'intermediate phenotypes' or endophenotypes, which they first introduced more than 30 years ago. Endophenotypes are subcomponents of the broader phenotype (e.g. alcoholism) that might bridge the gap between the heterogeneous disease and the more distal genotype. Endophenotypes represent processes (e.g. neuropsychological, biochemical, neurophysiological, neuroendocrine, cognitive etc.) underlying the complex disease that can be measured in the lab in a controlled fashion. Studying endophenotypes might lead to a more efficient identification of genes, because the gene might act more directly on this endophenotype (Dick \& Foroud, 2003). Several examples of endophenotypes that seem to distinguish between individuals who are more and less prone to developing alcohol or drug-related disorders are: the P300 amplitude reduction (e.g. Yoon et al., 2006), level of response (e.g. Schuckit \& Smith, 1996), sweet preference (e.g. KampovPolevoy et al., 1999), changes in heart-rate (e.g. Conrod et al., 2001), changes in beta-endorphin (Gianoulakis et al., 1996a, 1996b; Froehlich et al., 2000) and the stress response (e.g. Finn \& Justus, 1997). Recently, cue-elicited craving (e.g 
Hutchison et al., 2002a, 2002b), implicit memory associations (e.g. Stacy, 1997) and attentional bias (Franken, 2003) have been suggested as potential new endophenotypes. Once specific genes and endophenotypes have been identified, this can have implications for patient-treatment matching with respect to different types of medication (see below).

Not only biological factors such as $\mathrm{FH}$, heart-rate increase, the betaendorphin response, incentive sensitization, tolerance and specific and more general genetic factors can have an influence on drinking behavior, also cognitive factors can predispose an individual to develop alcohol-related problems.

\section{COGNITIVE FACTORS}

\section{Explicit alcohol expectancies}

M. S. Goldman's expectancy theory of alcohol states that the nervous system stores traces of it's own activation patterns. The template consists of a particular situation and the affective component that accompanied the situation (Goldman et al., 1999). Expectancies are already present before the onset of drinking alcohol (Dunn \& Goldman, 1996) and are shaped during life by genetic as well as environmental factors such as information about alcohol present in the media and other people that drink alcohol such as family and friends. Expectancies that people form about alcohol can be of several types: 1) people can drink because of positive reinforcement; that is, they expect to have more fun and sex after drinking alcohol, 2) they can drink because of negative reinforcement, to achieve tension reduction, 3) and they can have negative expectancies, for example when people get into a negative mood or become aggressive after drinking (Goldman et al., 1999; Goldman \& Darkes, 2004). According to Goldman, there is a final common pathway that leads to alcohol use and abuse through the expectancies or learned memory patterns a person has build up. These affective components that are stored in memory can be placed on two different dimensions; a positivenegative dimension (valence) and an arousal-sedation dimension (activation).

When people are explicitly asked to write down what they think alcohol makes them become, it turns out that light and heavy drinkers have different semantic networks, that is, different pathways that are activated after thinking about or drinking alcohol. Heavy drinkers first associate drinking with concepts related to positive arousal, such as funny and energetic (positive reinforcement) and light drinkers first relate the drinking of alcohol to sedation-related concepts such as sleepy, mellow and relaxed (Rather et al., 1992).

With the help of multidimensional scaling (MDS) these alcohol-expectancy words that people generate have been placed in a two-dimensional space (Rather 
\& Goldman, 1994). On the horizontal axis the words are arranged from left to right on the negative-positive dimension and on the vertical axis the words range from arousal, at the top, to sedation at the bottom. These two basic dimensions underlie the dimensions found for affective processing (Goldman et al., 1999). The hypothetical starting point of activation of the expectancy network for light drinkers is 'woozy' and for heavy drinkers it is 'energetic'. From there spreading of activation takes place towards other alcohol outcome expectancies. Since heavy drinkers also have a more tightly configured network (Rather \& Goldman, 1994), there is a very fast spreading of activation of concepts such as happy, talkative and horny, which will consequently lead to more drinking. In contrary, light drinkers first activate concepts related to the sedating effects of alcohol, such as woozy, relaxed and sleepy, which will in turn lead to more moderate alcohol use. Since the two groups of drinkers have had different types of experiences with alcohol, the stored information predicts different outcomes for them. Alcohol expectancies predict up to $50 \%$ of the explained variance of concurrent alcohol use (Goldman et al., 1999; Wiers et al., 1997). Furthermore, it has been shown that expectancies predict future drinking in adolescents after as much as 9 years (Stacy et al., 1991). However, after controlling for variables such as demographics, attitudes towards alcohol and previous alcohol use the amount of variance accounted for by expectancies drops below 10\% in most prospective studies (see Jones et al., 2001 for a review). Alcohol outcome expectancies have mainly been measured with explicit or direct measures such as expectancy questionnaires, which rely on self-report. In the past few years, however, so-called indirect or implicit measures have been developed that assess more automatic associations with alcohol.

\section{Implicit cognitions}

Implicit cognition means that "traces of past experiences affect some performance, even though the influential earlier experience is not remembered in the usual sense, that is, it is unavailable to self-report or introspection" (Greenwald \& Banaji, 1995, p. 4-5). In other words, behavior is partly guided by automatic processes that can exert their influence outside of conscious control. This automatic processing may partly explain why addictive behaviors remain persistent despite the disadvantages of the drug problem the addict is aware of at an explicit level (Wiers \& Stacy, 2006). Fazio and Olson (2003) introduced the MODE model (Motivation and Opportunity as DEterminants) in which they describe that an individual should have the motivation and be in the opportunity to inhibit the automatic response or action tendency. They suggest that processes are mostly mixed en not purely spontaneous or deliberate. It is the measure which is implicit (indirect) or explicit (direct) and not the attitude which is being measured. The implicit measure is supposed to 
capture the to-be-measured construct in a relatively unintentional or uncontrolled way. As such they might best tap into processes important to real-life behaviors such as addictive behaviors (De Houwer, 2006).

Several advantages of implicit measures over explicit measures should be mentioned: 1) implicit measures are less sensitive to socially desirable answering (Fazio \& Olson, 2003), 2) they might be able to measure processes the participant cannot introspect upon (Wilson et al., 2000), 3) they can explain an additional part of the variance, above the variance explained by explicit measures (Stacy, 1997), and 4) there is evidence that implicit and explicit memory are mediated by different neurobiological systems (Schott et al., 2005; Squire et al., 1993).

According to Goldman et al. (1999), alcohol expectancies are explicit at first, but become implicit after more experience. He states that in fact explicit and implicit cognition are qualitatively the same and that explicit and implicit measurements tap the same underlying construct. According to this theory, the use of implicit measures should be more accurate since people are not influenced by social desirability or the inability to express what they want to express. Stacy (1997), however, claims that explicit and implicit expectancies are two qualitatively different constructs that should be measured differently. According to the dual attitude model of Wilson, Lindsey and Schooler (2000), the implicit attitude is the default response that will be automatically or unconsciously given (for example under timepressure) and the (new) explicit attitude will take more conscious effort and capacity to be retrieved from memory. Stacy makes a clear difference between measuring explicit alcohol outcome expectancies and implicit memory associations. He also found that these two constructs had independent predictive effects on the use of alcohol and marihuana (Stacy, 1997). The fact that usually very low correlations between explicit and implicit measures are found (Hofmann et al., 2005) also can be interpreted to imply that these two are indeed different constructs as Stacy suggests.

In the past few years, repeated findings of dissociations between more explicit (controlled) versus more implicit (automatic) processes in social cognition and behavior have led to the development of several dual-process models. The model of Strack and Deutsch (2004) describes an Impulsive system and a Reflective system. The impulsive system is fast, bottom-up, requires little cognitive capacity, is based on automatic appraisal and behavioral schemata are activated by automatic spreading of activation in an associative network. It is not flexible in the sense that associations between the different elements in the long-term storage network change slowly. Conversely, the reflective system is based on short term storage, makes use of symbolic representations, operates slowly, is flexible, requires a high amount of cognitive capacity, can be easily disturbed and depends on intentions. It 
is involved in decision making and uses information from the impulsive system that is accessible. The systems both predict unique variance in behavior.

In the case of drug use, people are usually ambivalent, thus, there is a conflict between the impulsive and the reflective system (Deutsch \& Strack, 2006). Sensitization of the impulsive system (by repeated administration of the drug), states of deprivation or exposure to drug-related cues all increase the chance of activation of behavioral drug schemata in the impulsive system. This contributes to a heightened attention for the drug and (positive) thoughts about drug use which puts a person at risk for subsequent (compulsive) use. The reflective system can exert control over the impulsive system by for example the inhibition of motor responses or the allocation of attention. An example of a process belonging to the reflective system is explicit outcome expectancies of the effects of a drug (Goldman et al., 1999). Processes such as habits (Tiffany, 1990) and incentive sensitization (Robinson \& Berridge, 2003) are more related to the impulsive system.

Bechara and collegues (2006) propose a similar model to the functional model of Strack and Deutsch (2004), and they make suggestions for the underlying neural structures. In their model, the impulsive system is amygdala-striatumdependent and is involved in the reward or punishment of immediate actions. It responds to primary inducers (innate or learned stimuli that produce pleasurable or unpleasurable states), has been implied in incentive learning and chooses based on short term outcomes. The striatum has been found to be of importance at the implicit level. The individual responds with 'knowledge without awareness'. The reflective system is orbitofrontal-dependent and is involved in reward or punishment of future actions. It responds to secondary inducers (thoughts and memories of the primary inducers). Behavioral actions are based on long term outcomes. Three important brain regions have been found to be of importance to the reflective system. First, the anterior cingulate, which plays an important role in motor impulse control, thus 'willful' decisions based on 'knowledge with awareness'. Second, the orbitofrontal and dorsolateral prefrontal cortex, which are involved in perceptual impulse control. Third, the ventromedial prefrontal cortex, which has been implied in control over decision making. According to Bechara there are two possible causes of a loss of control or willpower which is characteristic of compulsive drug use: either a dysfunctional reflective system (with disturbed top-down processing of decision making and impulse control), or a hyperactive impulsive system (bottomup) which can gain control over cognition and behavior by 'hijacking' the reflective system. Short-term outcomes then prevail over long-term outcomes. A hyperactive amygdala-ventral striatum system results in an exaggerated processing of reward because structures such as the nucleus accumbens (NAc) become sensitized after 
repeated drug use (Robinson \& Berridge). Impulsive individuals are at a greater risk for addiction.

Broadly two types of measures have been developed that assess cognitive biases which can influence more automatic information processing in addictive behaviors: tests that measure memory associations and tests that measure attentional bias for or attentional distraction by drug-related stimuli. Implicit memory associations can be measured with tasks such as word association tasks (e.g. Stacy, 1997) or the Implicit Association Test (IAT, Greenwald et al., 1998; See Table 2.1 for an overview of several IATs that were used in this thesis). The IAT is a computerized categorization task in which stimuli belonging to either a target (e.g. alcohol vs soda) or an attribute category (e.g. arousal vs sedation) are classified by means of a left and right response key. The task consists of several phases and the IAT-effect is the subtraction of the two combination phases (both target and attribute stimuli are categorized at the same time), thereby providing a measure of the relative speed with which one combination (e.g. alcohol + arousal) is processed faster than the other combination (e.g. alcohol + sedation). The idea underlying the IAT is that concepts that are more strongly related in memory and share the same response key, are processed faster than concepts which are less strongly associated in memory and share the same response key (Greenwald et al., 1998).

Adaptations of the IAT have been applied to alcohol use (e.g. De Houwer ef al., 2004; Jajodia \& Earlywine, 2003; Palfai \& Ostafin, 2003; Wiers et al., 2002; 2005), cigarette smoking (Huijding et al., 2005; Robinson et al., 2005; Sherman et al., 2003; Swanson et al., 2001), cannabis smoking (Field et al., 2004), cocaine (Wiers et al., 2006) and gambling (Zack et al., 2005). General findings were that heavier drinkers associated alcohol more strongly with arousal on the ArousalIAT than light drinkers (Wiers et al., 2002), that approach associations on the Motivation-IAT correlated with binge drinking (e.g. Palfai \& Ostafin, 2003), that smokers revealed an implicit negative attitude towards smoking (e.g. Huijding et al., 2005), that non-cannabis smokers revealed a stronger implicit negative attitude towards cannabis smoking than the smokers (Field et al., 2004), that cocaine users had implicit positive and sedation associations (Wiers et al., 2006) and that problem gamblers showed a stronger association between alcohol and gambling wins than gambling losses (Zack et al., 2005). Similarly as with prospective studies on explicit expectancies, very few prospective studies with indirect measures have been conducted to predict addictive behaviors. Some studies have found predictive effects of word association tests above covariates such as previous use (Kelly et al., 2005) and explicit expectancies (Stacy, 1997). Implicit alcohol associations measured with (adaptations of) the IAT have been shown to predict alcohol use in stu- 
dents after 1 month (Wiers et al., 2002) and binge drinking in adolescents after 1 year (Thush \& Wiers, in press).

The second type of tests measures attentional bias for or attentional distraction by alcohol or drug-related stimuli and can be assessed with tasks such as the emotional Stroop task (see Cox et al., 2006 for a review). During this task, the participant is asked to respond only to the color a word is printed in. Some words are neutral (e.g. 'bus') and some words are alcohol-related (e.g. 'bar'). Emotionally relevant words grab the attention thereby slowing down the color-naming response. The difference between response latencies to the alcohol and neutral words is an indication of the attentional distraction an individual experiences by alcohol-related words while performing the task. In a small study by Cox et al. (2002) an increase in attentional distraction by alcohol-related stimuli in alcoholdependent patients in treatment predicted a worse treatment outcome. Also, smokers who showed more attentional bias were significantly more likely to lapse in the short-term (Waters et al., 2003). Similar findings were found for a large sample of heroin-dependent patients receiving treatment (Marissen et al., 2006). Clearly, it is hypothesized that tests such as the IAT and the emotional Stroop tap into components of the impulsive system rather than the reflective system. Nevertheless, they may also tap into different subprocesses, which has been suggested to explain the low correlations generally found between implicit measures (Bosson et al., 2000). An alternative explanation could be measurement error (Cunningham et al., 2001).

\section{RELATIONSHIP BETWEEN COGNITIVE AND BIOLOGICAL FACTORS}

How do the biological and cognitive factors described above relate to each other? The sensitized dopamine response in the brain reward system which is seen during the ascending limb after repeated alcohol use (and is even stronger in FHPs) might possibly be translated into alcohol arousal outcome expectancies at the explicit level and memory associations with arousal and approach at the implicit level (IAT). The same sensitized brain system attributes an incentive salience to alcohol and alcohol-related stimuli which can induce compulsive drug seeking behavior, craving and an attentional bias for alcohol-related stimuli (emotional Stroop). Genetically, individuals with specific polymorphisms (or genetic variations) of certain receptor genes (e.g. mu-opioid receptor gene (OPRM1), dopamine D4 receptor gene (DRD4) and the cannabinoid CB1 receptor gene (CNR1) amongst others) might show a differential response to alcohol due to a different brain receptor expression or a different interaction of alcohol with the receptor. This could in turn 
also be translated into different alcohol expectancies or responses in subjective arousal or craving at the cognitive level. FHPs for example, have been found to report stronger explicit positive alcohol expectancies than FHNs, especially for a high dose (Wiers et al., 2000). Similarly, FHPs tended to show stronger implicit arousal associations on the IAT than FHNs (Wiers, in press). A study by Erblich ef al. (2003) showed that FHPs reported a lower baseline stimulation score on a questionnaire and a stronger subsequent alcohol-induced increase in subjective stimulation compared with FHNs. This might point to a state of underarousal in FHPs which could be compensated with alcohol (or other drugs). These findings are similar to the low baseline levels of beta-endorphin and the stronger subsequent alcohol-induced increase of these endogenous opioids that are found in FHPs (Gianloulakis et al., 1996a, 1996b). The underlying biological mechanisms seem to be reflected and measurable at the cognitive level.

\section{GENES AND MEDICATION}

The presence of different genetic polymorphisms might have implications for patient-matching with respect to pharmacotherapeutic treatments. To name one example, naltrexone (Revia $\AA$ ) is a mu-opioid receptor antagonist which blocks the opioid receptors thereby reducing the subjective 'high' after alcohol consumption (Volpicelli et al., 1995b). It reduces craving for alcohol, prolongs periods of abstinence, decreases the number of drinking days and the drinks per drinking day (O'Malley et al., 1996; Volpicelli et al., 1992; Volpicelli et al., 1995a). It has been hypothesized that individuals with strong explicit, positive expectancies would benefit more from naltrexone treatment due to their possibly strong biological response to alcohol that results in these positive alcohol expectancies (Wiers et al., 2000). Montererosso et al. (2001) demonstrated that FHPs benefitted more from naltrexone treatment than FHNs, probably due to the blocking of their heritable and more sensitive beta-endorphin response. Additionally, patients carrying a copy of the $G$ allele of the mu-opioid receptor gene (OPRM1) relapsed significantly less often than patients homozygous for the A allele while being treated with naltrexone (Oslin et al., 2003). The $G$ allele has been associated with a differential mu-opioid receptor which has a potency to bind beta-endorphin three times more tightly (Bond et al., 1998). Thus, blocking the beta-endorphin response with naltrexone seems more efficacious in patients with a $G$ allele. Further, it has been suggested that individuals carrying a $G$ allele more often report a FHP (Ray \& Hutchison, 2004). Also, alcohol-dependent patients with a stronger sweet preference respond better to naltrexone (Kampov-Polevoy et al., 2004) and sweet preference has been associated with a FHP (Kampov-Polevoy et al., 2003). Thus, there seem to be as- 
sociations between a FHP, the $G$ allele of the mu-opioid receptor gene, an enhanced response to beta-endorphin, stronger sweet preference and a better response to naltrexone. This has implications for treatment-matching. Naltrexone could be the medication of first choice for patients carrying a copy of the $G$ allele. Possibly, individuals with other polymorphisms benefit more from treatment with other types of medication that target other receptor and neurotransmitter systems in the brain.

\section{OUTLINE OF THIS THESIS}

Chapter 2 investigates the relationship between implicit alcohol arousal and approach associations (measured with the Implicit Association Test) and attentional bias for alcohol-related stimuli (measured with an emotional Stroop task) on the one hand and alcohol-induced heart rate acceleration on the other hand, in 48 male heavy drinkers. It has been hypothesized that both the automatic cognitive biases and alcohol-induced heart-rate increase reflect processes related to incentive sensitization, therefore, we were interested if a positive relationship between these measures would exist.

Chapter 3 describes a study in which 67 alcohol-dependent patients performed the arousal-IAT, the approach-avoidance IAT and the emotional Stroop. It was hypothesized that the three measures would show a positive association. Furthermore, FHPs were expected to show stronger explicit arousal expectancies and implicit associations with arousal and approach than FHNs. In addition, a control group, consisting of 21 addiction clinic employees, was tested, to investigate whether the implicit cognitions would be specific for alcohol-dependent patients.

Chapter 4 describes a study in which treatment outcome (relapse or not) of 19 alcohol-dependent patients receiving naltrexone for 3 months was predicted by several explicit measures (craving, expectancies, motivation), implicit measures (arousal-IAT, approach-avoidance IAT, emotional Stroop) and several moderating variables (e.g. FH). It was hypothesized that FHPs would be more successful than $\mathrm{FHNs}$ at remaining abstinent due to the naltrexone treatment. Further, more exploratively, we were interested if changes in explicit and implicit variables would occur during the first six weeks of treatment in the subset of patients who did not dropout during this period.

Chapter 5 reports on a cue-reactivity study where 109 male heavy drinkers were exposed to water and their favorite alcoholic beverage. Subjective craving, subjective arousal and salivary reactivity were assessed. Furthermore, they were genotyped for the A1 $18 \mathrm{G}$ polymorphism of the mu-opioid receptor gene (OPRM1). It was hypothesized that $G$ allele carriers would respond with stronger cue reactivity 
than the individuals homozygous for the A allele. In addition, it was expected that the $G$ allele participants would more often report a FHP for alcoholism and more alcohol and drug use.

Chapter 6 describes a sub sample of 88 heavy drinkers of the cue-reactivity study described in Chapter 5 who were additionally genotyped on the variable number of tandem repeats (VNTR) of the dopamine D4 receptor (DRD4) and the rs2023239 polymorphism of the cannabinoid CB1 receptor (CNR1). It was hypothesized that individuals carrying the long allele of the DRD4 VNTR ( $>7$ repeats) and the individuals carrying a copy of the $C$ allele of the CNR 1 polymorphism would report more cue reactivity and more alcohol and drug use than the DRD4 short allele or CNR 1 TT allele participants, respectively.

Chapter 7 provides an overview and general discussion of the main findings in this thesis. Further, some clinical implications will be described, methodological issues will be raised and suggestions for further research will be provided. 



\section{CHAPTER 2}

\section{IS THE STRENGTH OF IMPLICIT ALCOHOL ASSOCIATIONS CORRELATED WITH ALCOHOL-INDUCED HEART-RATE ACCELERATION?}

Published as: Van den Wildenberg, E., Beckers, M., Van Lambaart F., Conrod P., \& Wiers, R. W. (2006). Is the Strength of Implicit Alcohol Associations Correlated with Alcohol-Induced Heart-Rate Acceleration? Alcoholism: Clinical and Experimental Research, 30, 1336-1348. 


\section{ABSTRACT}

Background: Heart-rate (HR) acceleration during the ascending limb of the blood alcohol curve has proven to be a reliable measure of the sensitivity to the activating effects of alcohol. In this study we investigated the correlation between an ethanolinduced cardiac change and the strength of implicit alcohol-related arousal and approach-associations and attentional bias for alcohol-related stimuli, in heavy drinkers. These three types of implicit alcohol-related cognitions have been proposed to reflect the strength of incentive sensitization that is experienced after repeated alcohol use.

Methods: 48 heavy drinking men performed a modified version of the Implicit Association Test (IAT; Greenwald et al., 1998) to measure their implicit alcohol arousal and approach-avoidance associations. A modified version of the emotional Stroop was used to measure attentional bias for alcohol-related stimuli (blocked and unblocked). Next, a high dose of alcohol $(1.0 \mathrm{ml} / \mathrm{kg}$ body weight of 95\% USP alcohol) was administered in a short period of time. Resting baseline Heart Rate (HR), Blood Alcohol Concentrations (BACs), mood and craving for alcohol were assessed before alcohol administration and for 2 hours post alcohol consumption.

Results: Contrary to our hypothesis, a negative association was found between implicit arousal-associations and alcohol-induced HR Change. This indicates that strong arousal-associations were correlated with a decrease in alcohol-induced HR. Approach-associations and attentional bias were not correlated with alcoholinduced HR Change, but both correlated positively with each other.

Conclusions: Alcohol-arousal associations and other implicit cognitions (attentional bias, approach associations) are not positively related to individual differences in the sensitivity to alcohol's activating effects, at least, not in the present sample consisting primarily of family history negative heavy drinkers.

Keywords: Implicit Alcohol Cognitions, Implicit Arousal associations, Implicit Approach associations, Attentional Bias, Alcohol-Induced HR Change. 


\section{INTRODUCTION}

Alcohol misuse and alcohol dependence are two of the most prevalent diagnoses of psychiatric disorders in the Netherlands. Almost 20\% of Dutch men develop lifetime alcohol abuse and 9.0\% develop alcohol dependence (Bijl et al., 1998). Approximately $90 \%$ of the Dutch population drinks alcohol, but obviously, the majority of this group does not become a problem drinker (Bijl et al., 1998; Van Dijck and Knibbe, 2005). The focus of this study is on possible mechanisms that are of importance in the development of alcohol problems. Not everybody is equally at risk. People show individual differences in their sensitivity to the effects of alcohol (Newlin and Thomson, 1991). These have been identified on several levels such as genetics, physiological responses, subjective responses and cognitions (see below).

One hypothesized marker of risk for alcohol problems is how a person responds to alcohol. Newlin and Thomson (1991) proposed a biphasic model with different risk factors. When blood alcohol concentrations (BACs) rise, individual differences in experiencing stimulating and positive effects are important. During the falling limb of the BAC curve negative and sedating effects become more prominent (Martin et al., 1993). First, a low level of reaction, or a reduced responsiveness during the declining limb of the blood alcohol curve has been found to be an important mediator in the risk of developing alcoholism (Schuckit and Smith, 1996). Because of tolerance during the falling limb, less impairment and less aversive consequences of drinking are experienced. It has been hypothesized that certain individuals, particularly those with a family history of alcoholism, are less likely to regulate their drinking pattern and more likely to develop alcohol problems because they experience fewer negative subjective and behavioral consequences of alcohol intoxication (Schuckit and Smith, 1996). In accordance with Newlin's (1991) model, there is also evidence suggesting that risk for alcoholism is associated with heightened subjective, physiological and biochemical responses to alcohol when blood alcohol levels are rising (Conrod et al., 1997; 2001; Erblich et al., 2003; Martin et al., 1993; Newlin and Thomson, 1991). The focus of the present article is on this stimulant reaction during the ascending blood alcohol curve. Individuals with a family history of alcoholism $(\mathrm{FH}+)$, especially men with a multigenerational family history of alcoholism and sensation seekers (Brunelle, et al., 2005), display a strong alcohol-induced Heart Rate (HR) acceleration after a high dose of alcohol during the rising limb of the blood alcohol curve (Conrod et al., 1997; Peterson et al., 1996). Alcohol-induced HR acceleration has proven to be a reliable and valid measure of the sensitivity to the psychomotor stimulant effects of alcohol (Conrod et al., 1997; 2001). 
According to the influential Incentive Sensitization Theory of Robinson and Berridge $(1993 ; 2000 ; 2003)$ the mesocorticolimbic reward system in the brain becomes sensitized after repeated alcohol use. Alcohol as well as alcohol-related cues become salient to the individual and can (unconsciously) activate the dopamine reward system, leading to psychomotor stimulation. This can be experienced as craving or "wanting". The individual displays a heightened state of attention for alcohol-related stimuli (an attentional bias) and shows more goal directed behavior to obtain the drug. So far the process of sensitized "wanting" had mainly been studied in animals. A second hypothesized system, "liking", is understood as mediating the pleasurable effects of the drug and is not subject to sensitization. It has been suggested that alcohol-induced HR acceleration in humans reflects individual differences in the sensitivity of the mesocortical reward system to alcohol and, thus, may reflect the degree to which an individual is susceptible to alcohol sensitization, or "wanting" with repeated administrations (Conrod et al., 1997; 2001). Given earlier work of Conrod et al. (1997; 2001) we hypothesized that alcohol-induced $H R$ increase could be a measure of sensitization in humans.

On a cognitive level, several mechanisms underlying alcohol use and misuse have been investigated. Alcohol expectancies can predict up to $50 \%$ of the variance in drinking behavior and also predict the development of problematic alcohol use (see Goldman et al., 1999b and Jones et al., 2001 for reviews). Based on multi-dimensional scaling, (most) expectancies can be placed in a two-dimensional space consisting of a valence dimension (positive-negative) and an arousal dimension (arousal-sedation). Heavy drinkers display positive arousal expectancies (e.g. "Drinking alcohol makes me energetic"), while light drinkers show sedation expectancies (e.g. "Drinking alcohol makes me sleepy"; Goldman et al., 1999b). Expectancies can be influenced by various distal influences such as genetics and family history, metabolism, personality factors and social environment (Goldman et al., 1999a). Individuals with special variants of e.g. the ALDH2 gene (McCarthy et al., 2001), D2 gene (Young et al., 2004) or that have a positive family history for alcoholism have alcohol expectancies (Wiers et al., 2000) that differ from those without a family history of alcoholism.

Expectancies have mainly been studied with explicit measures, that is, in a direct way by means of questionnaires. The past few years, however, new tools have been developed to assess automatic associations with alcohol. These so called implicit or indirect measures have certain advantages over explicit measures. First, they are less sensitive to socially desirable answers (Fazio and Olson, 2003). Second, they might be able to measure ongoing processes the participant cannot introspect upon (Wilson et al., 2000). Another reason to not solely rely upon explicit measures is the finding that implicit measures can explain an additional part of the 
variance in alcohol consumption above the variance explained by explicit measures (Stacy, 1997; Wiers et al., 2002). Fourth, explicit and implicit measures might tap into different underlying constructs (Stacy, 1997; Wiers et al., 2002). This is reflected in the fact that explicit and implicit measures usually show low correlations (Wiers et al., 2002; 2005). Furthermore, different brain structures seem to be involved in explicit and implicit processing: Explicit outcome expectancies seem to be mediated by cortical regions such as the prefrontal cortex, while implicit processes are primarily located in subcortical structures (Berridge, 2001; cf. Phelps et al., 2000).

In a study by Wiers and colleagues (2002) alcohol associations of heavy drinkers were assessed indirectly by means of an adapted version of the Implicit Association Test (IAT; Greenwald et al., 1998). The IAT is a categorization task that measures the relative strength of two target concepts (e.g. alcohol versus soda) with two attribute categories (e.g. positive versus negative). Stimuli that appear on the screen have to be categorized by means of two response keys (e.g. alcohol + negative words are assigned to the left response key and soda + positive words to the right response key). In a second combination phase the position of the target switches and is again combined with the attribute category (e.g. soda + negative to the left, alcohol + positive to the right). The IAT-effect is the difference in reaction times between both combination blocks (see Wiers et al., 2002; 2005 for a more detailed description). The assumption underlying the IAT is that the stronger the association between two concepts is represented in memory, the faster the reaction time will be (Greenwald et al., 1998; 2003). Results indicated that light and heavy drinkers both displayed a strong negative association with alcohol. On the arousal-sedation dimension, however, only the heavy drinkers demonstrated strong associations between alcohol and arousal. This seems to be an indication that the main difference between heavy and light drinkers is on the arousal dimension, heavy drinkers showing strong arousal-associations. These findings have been replicated in heavy drinkers (Wiers et al., 2005) and in alcoholic patients (De Houwer et al., 2004).

Palfai and Ostafin (2003) used a modified version of the IAT to measure implicit approach and avoidance tendencies in heavy drinkers and their relationship with drinking behavior. They found that strong approach-associations were positively correlated with binge drinking. Also, approach-associations were positively associated with the subjective anticipatory urge to drink alcohol and with arousal reactivity in a cue reactivity paradigm where the participant was exposed to his or her favorite drink. These findings of implicit arousal and approach-associations have been theoretically linked to the development of incentive sensitization (Wiers et al., 2002). Arousal as well as approach associations have both been found to 
correlate with subjective arousal and craving in a cue-reactivity paradigm (Krank et al., 2005; Palfai and Ostafin, 2003).

In the present study an adapted version of the IAT was used (Houben and Wiers, in press). Instead of measuring associations in a bipolar way (e.g. arousal versus sedation), which leads to a 'relative' IAT effect score, we measured the attribute categories in a unipolar fashion (e.g. arousal versus neutral, sedation versus neutral; cf. Jajodia and Earleywine, 2003). Since the effects of alcohol are biphasic, with activating effects when BACs are rising, and sedating effects when BACs are falling, it may be advantageous to assess both associations separately.

Other implicit measures such as the emotional Stroop task have been developed to measure attentional bias for alcohol-related stimuli (MacLeod, 1991; Williams et al., 1996). In the emotional Stroop task participants respond to the color the word is printed in while ignoring the contents of the word. Emotionally relevant stimuli grab the attention, which interferes with the color naming response. This leads to slower reaction times. In an eye-movement study with a pictorial dot-probe task for smokers (Mogg et al., 2003) it was found that mainly attentional disengagement from the emotionally relevant stimulus was difficult. To alcohol dependent patients, alcohol-related words can be seen as emotionally relevant stimuli. Therefore their response tends to be slower to alcohol-related words compared to neutral words (Johnsen et al., 1994; Sharma et al., 2001; Stormark et al., 2000). Attentional bias for alcohol-related stimuli in heavy drinkers has only been found after some form of priming the participant: a sip of their favorite drink (Jones and Schulze, 2000), priming with an alcohol beverage word (Kramer and Goldman, 2003), exposure to the sight and smell of an alcoholic beverage (Cox et al., 2003) or exposure to alcohol advertisements (Cox et al., 1999). In alcoholics, however, an emotional Stroop effect has been found repeatedly without priming (for reviews: Cox et al., 2006; Bruce and Jones, 2006). Attentional bias for alcohol-related stimuli has also been linked to the development of incentive salience (Franken, 2003). Hence, an attentional bias for alcohol, implicit arousal (Wiers et al., 2002) and approach-associations (Palfai and Ostafin, 2003) have all been theoretically linked to the development of sensitized "wanting", likely reflecting the cognitive representation of incentive motivation for alcohol consumption.

In conclusion, the present study investigates the relationships between biological and cognitive measures of psychomotor stimulation in heavy drinkers. Alcoholinduced $H R$ increase seems to reflect the sensitivity to psychomotor stimulation during the ascending BAC curve (Conrod et al., 1997, 2001). We hypothesized that alcohol arousal and approach-associations (measured with the IAT) and attentional bias for alcohol-related stimuli (measured with the emotional Stroop) might reflect sensitized "wanting" in humans. Therefore, it was expected that these three 
cognitive constructs would show a positive correlation with HR increase after the rapid consumption of a high dose of alcohol. Furthermore, we expected the three implicit measures to correlate positively with each other since they might all tap into the underlying mechanism of incentive sensitization.

\title{
MATERIALS AND METHODS
}

\section{Participants}

Participants were 48 male heavy drinkers (mean age $=20.4$ years, $S D=3.5$ ) who were recruited via flyers at Maastricht University, several Colleges and pubs. They were subsequently screened by telephone on several inclusion and exclusion criteria. The main inclusion criterion was a mean alcohol consumption of 15 alcoholic standard consumptions per week and at least 1 binge episode in the past 2 weeks. Age range was between 18 and 45 years. 18 is the minimum age required for legal drinking in the Netherlands and 45 was used as upper limit to exclude individuals with possible heart problems. Exclusion criteria were the regular use of drugs other than alcohol and cigarettes, any other native language than Dutch, medical conditions such as liver problems, diabetes or heart complaints, the use of medication for which alcohol consumption is contraindicated, dyslexia, colour blindness, psychiatric problems in the past and a positive family history of schizophrenia, depression, bipolar disorder or suicide. One participant indicated not being from Dutch origin, but was included in the analyses since he was a native Dutch speaker. After completion of the experiment participants received a monetary compensation of $€ 40$ (equivalent to $\$ 45$ ).

\section{Materials and Measures}

\begin{abstract}
Alcohol Use
Alcohol use was measured with a quantity-frequency questionnaire (Wiers et al., 1997) based on the Time Line Follow-Back method (Sobell and Sobell, 1990). Participants indicated how many drinks they had had on each day of the previous week and if it was more, less or equal to what they typically drank on that particular day of the week. From this information the average alcohol consumption per week could be calculated. Furthermore they filled out how many binges (defined as 5 drinks or more in a drinking episode) they had had in the past 2 weeks. Mean weekly consumption was 33.8 standard drinks (SD $=14.5)$. Mean number of binges in the past 2 weeks was $4.8(S D=2.17)$.
\end{abstract}




\section{Alcohol-Related Problems}

The Alcohol Use Disorders Identification Test (AUDIT; Saunders et al., 1993) was used to screen for the development of possible alcohol problems. The 10 items question consumption (first 3 items) and problems related to alcohol dependence (other 7 items). Mean AUDIT score of our sample was 13.6 (SD = 4.5). According to Fleming et al. (1991) a cut-off score of 11 should be used in college samples as an indication of alcohol abuse. The internal consistency of the AUDIT was 0.77 (Cronbach $\alpha$ ). Furthermore the 18-item version of the Rutgers Alcohol Problem Index (RAPI; White and Labouvie, 1989) was used, which correlates 0.99 with the 23-item version (White and Labouvie, 2000). Participants indicated on a 5-point Likert scale how often several alcohol-related problems had occurred. Mean score was 19.7 (SD = 8.5) and the internal consistency was 0.73. The AUDIT and RAPI questionnaires correlated highly, $r=0.74$.

Two variables were composed to represent general measures of alcohol use and problems. The variable Alcohol Use consisted of the sum of the z-transformed scores of the mean alcohol consumption per week and the first three AUDIT items. The variable Alcohol Problems was calculated by adding the z-transformed scores of the remaining 7 AUDIT items and the RAPI sum score.

\section{Explicit Alcohol-Related Cognitions}

Expectancies about the arousing and sedative effects of alcohol were assessed using 10-cm Visual Analogue Scales (VAS). The questionnaire consisted of 11 Arousal and 13 Sedation VAS items. These 24 expectancy items were derived from two separate measures: The Biphasic Alcohol Effects Scale (BAES; Martin et al., 1993) and the arousal and sedation IAT (as has been done in previously by Houben and Wiers, in press; Wiers et al., 2002; 2005). Of this total of 28 items, 4 appeared in both measures and were thus excluded, leaving a final 24-item VAS Expectancy questionnaire. All 24 items were presented twice, once participants were questioned about their expectancies immediately after drinking a high dose of alcohol (e.g. "Directly after drinking a high dose of alcohol I become energetic") and the second time they indicated their expectancies 1.5 hours after drinking a high dose of alcohol (e.g. "1.5 hours after drinking a high dose of alcohol I become energetic"). The scale ranged from 'Absolutely disagree' $(0 \mathrm{~mm})$ to 'Absolutely agree' (100 mm). Both VAS questionnaires were filled out before alcohol administration. 


\section{Implicit Association Test}

All IAT's were programmed in ERTS 3.18 (Beringer, 1996). The target and attribute categories both consisted of 6 exemplars each. The target categories were labeled 'alcohol' and 'soda'. The five unipolar attribute categories were labeled 'pleasant' (positive), 'unpleasant' (negative), 'active' (arousal), 'quiet' (sedation) and 'materials' (as irrelevant control block), all versus the category 'neutral'. The bipolar block carried the labels 'to approach' versus 'to avoid'. The stimuli used in the five unipolar IATs were all rated by different participants $(N=68)$ in a pilot study, on valence, arousal and familiarity and were subsequently selected. The positive, negative and neutral substantives used in the two valence IATs were matched on familiarity, word length and number of syllables and were on average neutral with respect to arousal (e.g. 'peace', 'depression' vs. 'circle'). For the arousal and sedation blocks adjectives were used, also in the opposing category 'neutral' (e.g. 'talkative', 'relaxed' vs. 'constant'). These were also matched on familiarity, word length and number of syllables and were on average neutral with respect to valence. All four IATs (positive, negative, arousal and sedation) had a fixed 'neutral' category it was paired with. The stimuli that were used can be found in the Appendix. The presentation of the four unipolar attribute dimensions was partially counterbalanced according to a Latin Square design, resulting in four possible orders. The bipolar approach-avoidance IAT was always on the fifth position, because it was of a more exploratory nature. Stimuli of the approach-IAT were only matched on word length. Matching on valence seems impossible since approach words always tend to be rated as positive and avoidance words as negative. The presentation of the irrelevant control IAT 'materials' always came last. These words were also matched with the neutral category on valence, arousal and familiarity.

The standard IAT consists of 5 phases (see Table 2.1). In the first phase the target categories 'alcohol' and 'soda' were practiced (e.g. 'beer', 'juice'). This phase was only presented once, as the first phase of the first IAT. IATs 2 to 6 were made up of 4 phases. In the target phase, all 6 alcohol and 6 soda stimuli were presented twice, resulting in a total of 24 trials. The second phase was the introduction of the attribute categories (e.g. 'pleasant' versus 'neutral'). Again, all 6 pleasant stimuli and all 6 neutral stimuli were presented twice, leading to 24 trials. In the third IAT phase, the first combination phase (C), the target categories as well as the attribute categories were presented. Of all four categories each stimulus was presented twice, with a total of 48 trials. In the fourth phase the attribute categories were reversed (e.g. 'neutral' vs 'pleasant') with a total of 24 trials and the final phase was the reversed combination phase (R) in which the targets and reversed attributes were again combined (48 trials). Order of the two combination blocks was counterbalanced, with half the participants starting with the easier Combina- 


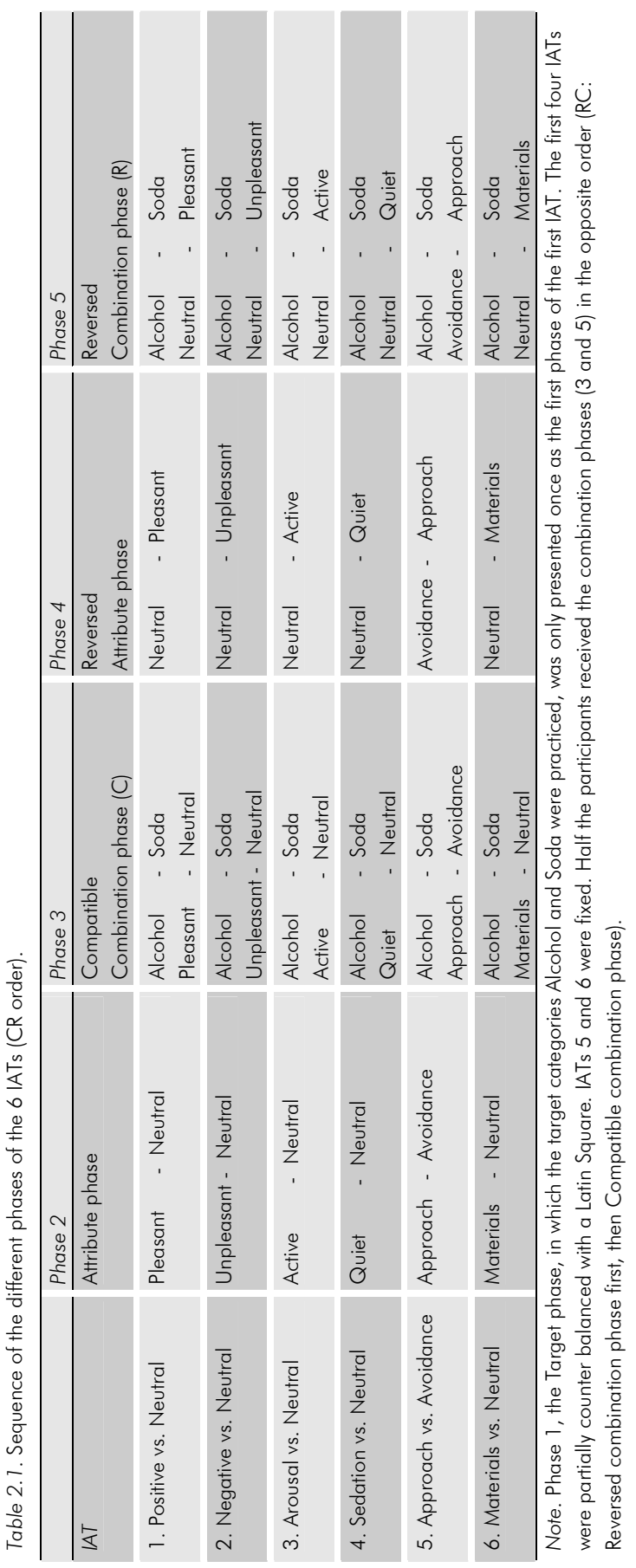


tion phase (CR) and the other half starting with the more difficult Reversed Combination phase (RC). During performance of all IAT blocks, the category labels were presented in the left and right upper corners of the computer screen and stayed there during the entire task. Stimulus words were presented in the middle of the screen in black against a light grey background. The stimulus word remained on the screen until a response was generated. The intertrial interval was 250 milliseconds. In case of an incorrect response the feedback 'wrong' was presented in red letters under the stimulus word. When a participant was too fast ( $<300 \mathrm{~ms}$ ) or too slow (>3000 ms) the messages 'too fast' or 'too slow' appeared on the screen. Reaction times and errors of all blocks were recorded. Reliabilities of the IAT blocks were calculated by correlating the practice trials (first 16 trials) with the test trials (last 32 trials) of the combination blocks. Subsequently, this correlation was entered in the Spearman Brown formula leading to Cronbach's $\alpha$ of the test. The internal consistencies were as follows: Approach-Avoidance: -0.01 , Materials: 0.40 , Positive: 0.62, Sedation: 0.64, Negative: 0.72 and 0.76 for the Arousal IAT. The unipolar IAT has been found to show somewhat lower reliabilities compared with the bipolar IAT (Houben and Wiers, in press; Wiers et al., 2005), which usually displays as of $>0.65$. Compared with other indirect measures, however, the IAT generally displays reasonably good internal consistencies (Bosson et al., 2000).

\section{Emotional Stroop task}

The Stroop task was presented on a computer and consisted of four blocks. The subject was required to respond with one of four different response keys, representing the four colors that were used. Colors were indicated on the response keys. Before each trial a fixation cross appeared for $500 \mathrm{~ms}$, to maintain the attention in the middle of the screen. In the first block 40 practice trials were offered. These were ten different non semantic strings such as@@@@@ and $\$ \$ \$ \$ \$$. Every stimulus was practiced once in each of the four colors: red, yellow, green and blue. The second block was a quasi random or mixed presentation of three stimulus word categories: neutral, alcohol-related and color. The presentation of a stimulus was never followed by a stimulus word from the same category. Neutral words were semantically related, belonging to the category 'traffic'. Four specific words were used: "car," "bus," "bike" and "plane", and four general words: "bridge," "asphalt," "railroad" and "station". The alcohol-related category also consisted of 8 stimulus words: "wine," "beer," "vodka" and "gin" being specific, and "pub," "drink," "bar" and "liquor store" as general words. Stimuli from the neutral and alcohol-related categories were matched on number of syllables. Each stimulus word of these two categories was presented once in every color, leading to a total of 32 trials per category. The four color words were used as a third category. Each 
color word was presented twice in every color with a total of 32 trials. The total number of trials in the mixed block was 96. After a short break of 1 minute the third block was presented in which the 8 neutral stimuli were all presented in a blocked fashion. Each word was presented once in every color. The fourth block was the blocked presentation of the alcohol words. Again, all 8 alcohol stimulus words were presented once in every color, leading to a total of 32 trials. In case of an incorrect response the feedback 'wrong' appeared on the screen. If the participant was too slow (>3000 ms) the message 'faster' was presented. Errors and reaction times of all four blocks were recorded.

\section{Mood}

The Profile of Mood States (POMS; McNair et al., 1971) and Biphasic Alcohol Effects Scale (BAES; Martin et al., 1993) were used to measure changes in mood. The short version of the POMS consisted of 5 subscales: Tension, Anger, Vigor, Fatigue and Depression. Participants indicated on a 5-point Likert scale how strong certain mood states were present at that particular moment. The BAES measured alcohol-induced mood changes and consisted of 2 subscales, Stimulation and Sedation, both containing $710-\mathrm{cm}$ VAS items.

\section{Craving}

On a 10-cm VAS, craving for alcohol was measured, once before and 8 times after alcohol administration. Participants were asked to respond to "At this moment I feel...", with a response format ranging from 'Absolutely no urge to drink' (0 mm) to 'An almost irresistible urge to drink' (100 mm). The use of a single VAS to measure craving has proven to be useful (Kozlowski et al., 1996). Cronbach's a over the 9 time measurements was 0.95 .

\section{Heart Rate}

HR was recorded with the Polar S810. The Polar watch recorded the HR every 5 seconds from two sensors on the chest band that were located bilaterally on the participant's chest. Of the 10 minute baseline recording, the most artefact-free 60sec period was selected and the average was chosen to reflect sober resting HR. After alcohol consumption HR was sampled every 5 seconds for 2 consecutive hours. Mean scores per 15 minutes were obtained by averaging the most artefactfree 3-min. period per 15 minutes (Conrod et al., 1997). In total 1 sober resting $\mathrm{HR}$ and 8 post alcohol resting HR averages were obtained. 


\section{Blood Alcohol Concentrations}

BACs were determined by using a Lion Alcolmeter SD-400. Participants were asked to breathe strongly and constantly for several seconds until the breathalyzer signalled that the recording was correct. BACs were recorded before drinking and every 15 minutes after alcohol consumption. The variable Latency to peak BAC was calculated from these measurements.

\section{Procedure}

Participants entered the lab either at 15.00 or at 17.00 in the afternoon. They had been asked to refrain from alcohol for 24 hours before the start of the study. Furthermore they were asked not to eat or drink any coffee 2 hours before the experiment started. They signed the informed consent form and then performed the IAT and Stroop task in a balanced order. Before each task they were sip primed for 30 seconds, with their favourite drink. They were asked to take a good look at the drink, smell it and then take one sip. After performing the implicit measures participants filled out several questionnaires. Participants then went to another lab where the second part of the experiment was conducted. Their weight was measured for correct alcohol dosage administration. When the Polar watch and chest band had been placed in the correct position, the participant was seated and the 10 minute baseline HR recording was started. After baseline recording, each participant received $1.0 \mathrm{ml} / \mathrm{kg}$ body weight of $95 \%$ USP alcohol. Alcohol was administered in the form of 5 'shots' of frozen $40 \%$ vodka, based on the procedure of Conrod et al. (1997). At the rate of 1 shot per minute, the total amount of alcohol was consumed within 5 minutes. Immediately after the final shot had been consumed the Polar watch started recording again, for 2 consecutive hours which the participant spent in silence, reading. Every 15 minutes BACs were recorded as well as mood (BAES) and craving for alcohol. The POMS was only administered twice after alcohol administration (after 30 and 90 minutes). After 2 hours the HR recording was stopped and the participants received a hot meal. They stayed in the lab until their BACs had dropped to the 0.05 level after which they were brought home with a taxi. The study was approved of by the Ethics Committee of the Department of Psychology of Maastricht University.

\section{RESULTS}

The ultimate goal of this study was to investigate the associations between alcoholinduced changes in $\mathrm{HR}$, subjective mood and craving on the one hand and measures of explicit and implicit cognition on the other hand. First, we will describe the 


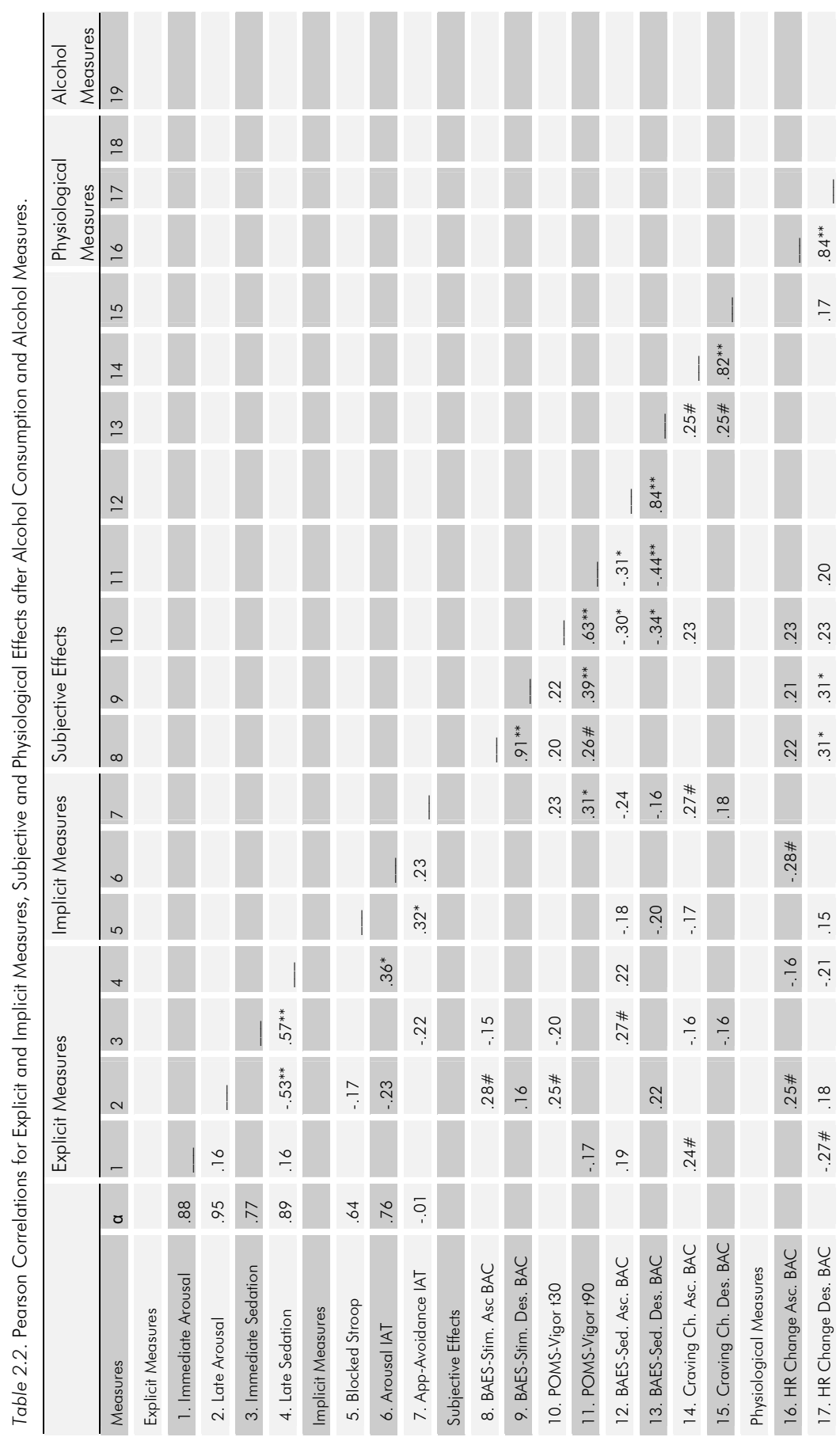




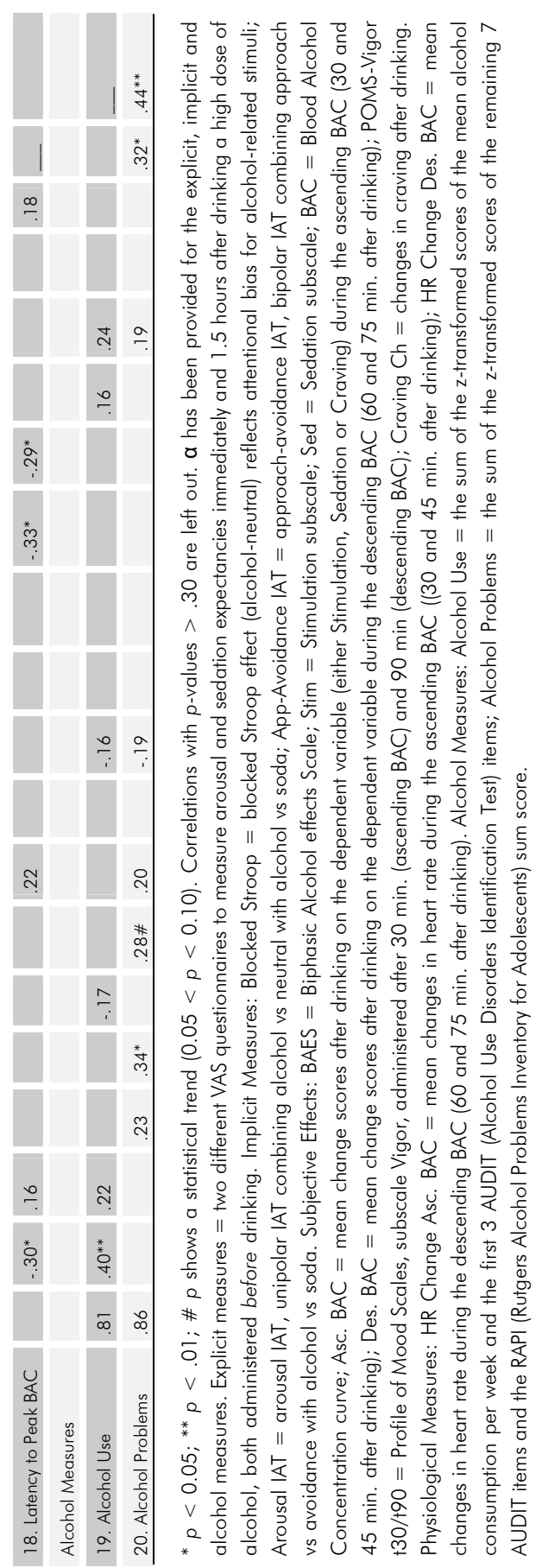


results of the explicit expectancies and their associations with other measures of interest. Second, the different implicit cognition tests will be presented and with which measures these were correlated. This will also include the main hypothesis of whether or not the implicit associations were associated with alcohol-induced HR change. Third, the physiological and subjective effects after rapid alcohol consumption will be described and their associations with other variables. Correlations between the different variables can be found in Table 2.2.

\section{Explicit Alcohol-Related Cognitions}

Mean immediate and late arousal expectancies were respectively 61.21 (SD = 13.27) and 52.26 (SD = 18.82), which indicated the heavy drinkers expected above average arousal from drinking a high dose of alcohol (the VAS ranged from 0-100 mm). The difference between immediate and late arousal expectancies was significant $(t(47)=2.93, p=0.005)$, indicating that participants expected to be less aroused 1.5 hours after drinking than immediately after drinking. Mean immediate and late sedation expectancies were respectively 34.31 (SD $=11.55$ ) and 45.48 (SD = 17.01), indicating that participants expected below average sedation from drinking a high dose of alcohol. A t-test revealed that the difference between immediate and late expectancies was significant $(t(47)=-5.49, p=<0.001)$. Thus, participants expected to become more sedated 1.5 hours after drinking a high dose of alcohol. All 4 scales showed good internal consistencies (See Table 2.2).

Correlations involving Explicit Immediate Arousal and Late Sedation Expectancies

As can be seen in Table 2.2, the immediate arousal expectancies correlated significantly with Alcohol Use $(r=0.40, p=0.005)$ and Latency to peak BAC ( $r=$ $0.30, p=0.04)$. This indicates that heavy drinkers with strong arousal expectancies immediately after drinking a high dose of alcohol, consume large amounts of alcohol (even within this sample of heavy drinkers), and reach their peak BAC relatively fast. The late sedation expectancies showed a significant correlation with Alcohol Problems ( $r=0.34, p=0.017)$, indicating that participants with strong sedation expectancies 1.5 hours after drinking, experienced more alcohol-related problems compared to participants with less strong late sedation expectancies. 


\title{
Implicit Alcohol-Related Cognitions
}

\author{
Implicit Association Test
}

Results of the Implicit Association Test are presented in Figure 2.1. Overall percentage of errors over all 6 blocks was $4.9 \%$. For matters of interpretation, the conventional IAT-effects in milliseconds (Greenwald et al., 1998) are graphically represented. Analyses in the text will describe the new D600 algorithm as advised by Greenwald et al. (2003). According to the new D600 algorithm, practice trials are also included in the analysis and the data are standardized at the level of the participant. It will be indicated when differences were found between the conventional measure in milliseconds and the D600 measure.

IAT-effects are always difference scores, where the reaction times from the C (Combination) phase are subtracted from the reaction times of the $R$ (Reversed combination) phase (R-C). If responses are faster in the compatible $C$ phase than the incompatible $R$ phase, the IAT-effect will be positive. Participants performed all 6 IAT blocks in one of the two variants of the IAT, CR or RC, where the CR version usually yields larger effects (cf. Greenwald et al., 1998; Wiers et al., 2005). For some affective dimensions results differed between the two versions (e.g. Positive). Therefore, the IAT data are presented per version if differences were found as well as aggregated over versions.

One-sample t-tests indicated significant IAT-effects for the Negative, Arousal and Materials (control) blocks in both the CR and RC condition. The largest IATeffect was found for the Negative block, indicating much stronger associations between alcohol and negative compared with alcohol and neutral, $t(47)=13.69$, $p<0.001$ for the group as a whole. The same pattern was found for Arousal, $t(47)=7.70, p<0.001$ : Participants showed a stronger association between alcohol and arousal than between alcohol and neutral. The IAT-effect of the Materials control block was significant in both groups using the D600 measure, $t(47)=$ 5.64, $p<0.001$, but only reached borderline significance in the RC condition using the conventional algorithm $(t(23)=2.00, p=0.06)$. The IAT-effect of the Positive block was found to be significant in the $C R$ condition only, $t(23)=6.55, p$ $<0.001$. No significant IAT-effects were found for the bipolar ApproachAvoidance block and the unipolar Sedation block for any of the groups or the two algorithms. 


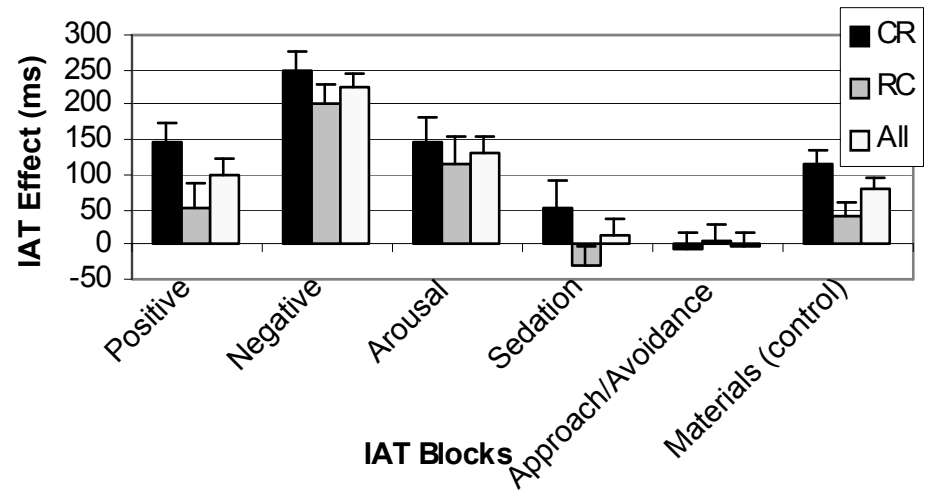

Figure 2.1. IAT-effects for the unipolar (versus neutral) blocks: Positive, Negative, Arousal, Sedation and Materials (control) and the bipolar Approach-Avoidance block. An IAT-effect is the difference in reaction times between the compatible combination phase (C) and the reversed combination phase ( $R$ ). If the response is faster in the compatible $C$ phase (e.g. alcohol + active) than the incompatible R phase (alcohol + neutral) the IAT-effect will be positive (R-C). Reaction times are represented according to the conventional algorithm in ms. (Greenwald et al., 1998). Half the participants performed all IATs in CR order (starting with the easier compatible $C$ phase, followed by the more difficult reversed $R$ phase). The other half performed the RC order. Usually IAT-effects are stronger in the CR order. When both orders were combined (all participants) significant IAT effects were found for the Negative, Arousal and Materials block.

\section{Emotional Stroop Task}

Only correct responses were used in the Stroop analyses, both for the unblocked (mixed) and blocked version. Overall error rate for all the trials was $4.5 \%$. Mean reaction times for the three word categories in the mixed block were $651.2 \mathrm{~ms}$ (SD $=141)$ for the neutral words, $662.0 \mathrm{~ms}(S D=156)$ for the alcohol words, and $702.8 \mathrm{~ms}(S D=182)$ for the colour words. All Stroop analyses given below were run on the log transformed data in order to achieve a better normal distribution. Paired t-tests revealed that the difference between neutral and alcohol words on the one hand, and colour words on the other hand, were significant ( $p s<0.005$ ). However, the emotional Stroop effect, the difference in reaction times between alcohol and neutral words was non-significant $(t(47)=1.23, p=0.22)$. To further investigate the influence of a preceding colour word on the reaction time of the target word (neutral or alcohol; cf. Waters et al., 2003) the data of the mixed block were reanalysed (see Table 2.3 for the raw reaction times). The difference in 
reaction times between neutral and alcohol words preceded by a colour word was non-significant $(p>0.50)$. Since there was no meaningful variability in attentional bias, as measured with the mixed Stroop, this measure will not be included in further analyses.

Analysis of the blocked presentation of the neutral and alcohol-related words did reveal a significant emotional Stroop effect. Mean reaction times for the words in the neutral and alcohol block were respectively $606.4 \mathrm{~ms}(S D=127)$ and $634.3 \mathrm{~ms}(S D=146.7)$. A paired t-test showed that the difference between these two conditions was significant $(t(47)=2.45, p=0.018)$.

Table 2.3. Mean reaction times (and SDs) of the neutral, alcohol and colour words as a function of the word preceding the target word on the previous trial.

\begin{tabular}{l|c|c|c}
\hline & \multicolumn{3}{l}{ Target Word } \\
\cline { 2 - 4 } Word Before & Neutral & Alcohol & Colour \\
\hline Neutral & $X$ & $612.1(248)$ & $729.8(348)$ \\
\hline Alcohol & $626.0(200)$ & $X$ & $716.8(268)$ \\
\hline Colour & $656.2(160)$ & $677.2(268)$ & $X$ \\
\hline
\end{tabular}

Note. A target word was never preceded by a stimulus from the same word category $(X)$.

\section{Correlations between Implicit, Explicit and Alcohol Measures}

As expected, a positive correlation between the Arousal IAT and Alcohol Problems ( $r=0.28, p=0.029$, one-tailed) was found, as has been found in previous research (Houben and Wiers, in press; Wiers et al., 2002). Neither of the 2 IATs of main interest correlated significantly with Alcohol Use. This might be due to the restricted range of drinking behaviour in this sample of heavy drinkers. The hypothesized correlation between alcohol Arousal and alcohol Approach IAT-effects (both theoretically related to incentive sensitization) was found to be in the expected direction, but was not significant $(r=0.23, p=0.12)$. The Arousal and Approach IATs did not correlate significantly with the other 4 IAT measures. The Approach IAT tended to be correlated with changes in craving experienced during the ascending BAC ( $r=0.27, p=0.06)$. The blocked Stroop was found to be positively associated with approach-associations on the IAT $(r=0.32, p=0.028)$, as hypothesized. Attentional bias was unrelated to Alcohol Use and Problems. Consistent with earlier findings (Wiers et al., 2005), implicit arousal associations were positively correlated with late sedation expectancies as indicated on the VAS questionnaire $(r=0.36, p=0.01)$. 


\section{Correlations between Implicit Measures and Alcohol-Induced HR Change}

Heart rate acceleration was defined as the change in post-alcohol HR compared to pre-alcohol (post alcohol cue) HR Baseline (HRB). Since HRB (pretest) tended to correlate positively with the Arousal IAT score $(r=0.24, p=0.10)$ ANOVA of change is preferred over ANCOVA in which the absolute HR post test scores would be covaried with HRB (Van Breukelen, 2006). HR Change during the ascending BAC curve was calculated by averaging the mean HR Changes after 30 and 45 minutes (correlation of 0.81). Contrary to our hypothesis, none of the three implicit measures correlated positively with alcohol-induced HR change. If anything, arousal associations were inversely related to alcohol-induced HR change during the ascending BAC ( $r=-0.28, p=0.053)$. After partialling out the effect of Alcohol Problems, the correlation was found to be $-0.31(p=0.034)$. Within the group of participants with strong arousal associations (based on a median split) the Arousal IAT correlated -0.43 ( $p=0.042$ ) with HR Change (controlled for Alcohol Problems). Thus, unexpectedly, individuals with stronger implicit arousalassociations tended to show a HR decrease after alcohol consumption.

As expected of a sample of individuals without family histories of alcoholism, mean HR hardly increased in response to alcohol consumption (a mean maximum increase of $1.6 \mathrm{Bpm}$, see Figure 2.2A, all participants; Consistent with Conrod et al., 1997). However, due to the heavier drinking nature of this sample, we did expect to find some HR responders. Based on mean HR \pm 1 SD 11 high HR responders were identified and 8 low HR responders. An independent t-test revealed that these two groups differed significantly with respect to their arousal associations, $t(17)=2.14, p=0.047$. High HR responders had an average arousal IATeffect of $108 \mathrm{~ms}$, whereas low HR responders had an IAT-effect which was twice as big $(215 \mathrm{~ms})$. Hence, these three analyses ((partial) correlation, median split and extremes) all indicate that instead of the expected positive association between the Arousal IAT and alcohol-induced HR increase, the reverse pattern was found.

A possible reason for the limited range of $\mathrm{HR}$ response was that a subgroup of participants had a relatively high $\mathrm{HRB}$, leaving little room for increase. A median split of HRB (65 Bpm) was used to divide the participants. In accordance with this (post-hoc) hypothesis low HRB participants showed a HR increase, significantly different from 0 at all measurement moments ( $p s<0.02$ ) with highest values during the ascending limb of the BAC curve (see Figure 2.2A). In contrast, high HRB participants showed a HR decrease, significantly different from 0 on all measurements except 15 and 75 minutes ( $p s<0.05$ ). This may suggest that there was a ceiling effect on alcohol-induced HR increase. 
2A) HR Change

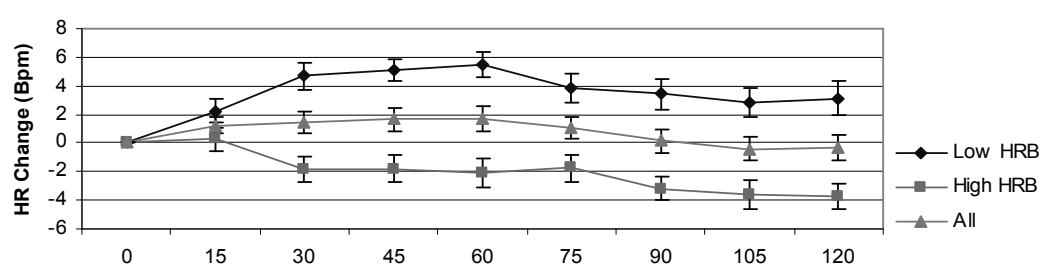

2B) BAC

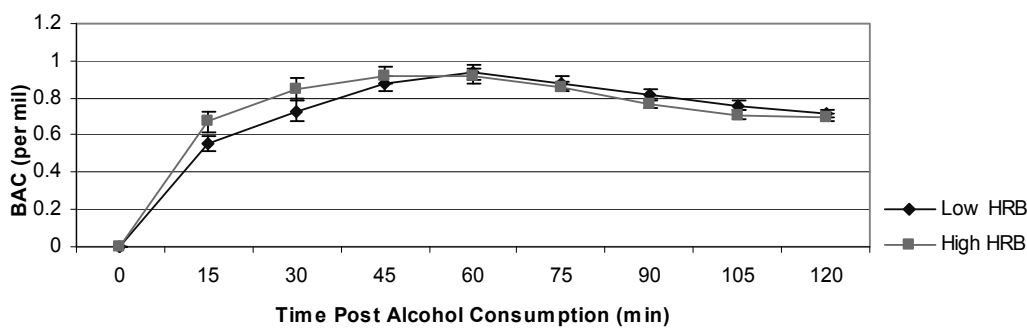

Figure 2.2. After alcohol administration, changes in Heart Rate (HR, 2A) and Blood Alcohol Concentrations (BACs, 2B) were measured for 2 consecutive hours. Based on a median split of Heart Rate Baseline (HRB) participants were divided into two groups (Low and High HRB). Different patterns in HR Change emerged for both groups: participants with a Low HRB showed an alcohol-induced HR increase, while participants with a high HRB showed a decrease in HR. No significant differences were found between these groups with regard to their BACs.

\section{Physiological Effects and Subjective Mood Effects after Alcohol Con- sumption}

As expected BACs rapidly increased after alcohol intake (Figure 2.2B) reaching a maximum of approximately 0.9 per mil after 45-60 minutes. HR Change was not significantly correlated with BACs during the ascending limb ( $p s>0.40$ ).

Changes in subjective mood were calculated by averaging the mean Stimulation as well as Sedation scores on the BAES 30 and 45 minutes after drinking (for the ascending BAC curve), corrected for baseline mood. The average of 60 and $75 \mathrm{~min}$ represented the mood changes during the descending limb. Furthermore, the scores of the Vigor subscale of the POMS 30 and 90 min after drinking were used. All mood changes were subjected to correlation analysis with the physiologi- 
cal changes in HR and Latency to peak BAC. During the ascending limb, Vigor scores tended to correlate positively with HR Change, but non-significantly ( $r=$ $0.23, p=0.12$ ). No significant correlation was found between the Stimulation subscale of the BAES and HR Change. This could be due to the unexpected finding that, compared with baseline stimulation scores, there was, on average, no evidence of an increase in subjective stimulation during the rising BAC curve (ps > 0.60). This, in turn, could be related to the fact that only a minority of the participants displayed an alcohol-induced HR increase during the ascending limb. The Sedation scores of the BAES correlated negatively with Latency to peak BAC ( $r=$ $0.33, p=0.024)$, indicating that participants who reached their peak BACs fast, experienced more subjective sedation. During the descending limb, the BAES Stimulation scores correlated significantly with HR Change $(r=0.35, p=0.016)$. Participants with a HR increase showed stronger subjective stimulation. This effect was not found for the Vigor subscale of the POMS.

\section{DISCUSSION}

This study investigated the associations of implicit arousal-associations, approachassociations and attentional bias on the one hand, with alcohol-induced changes in heart-rate, craving and subjective mood on the other hand. The main findings can be summarized as follows: First, it should be noted that not all participants showed an alcohol-induced HR increase. Participants with a low HR Baseline (HRB) showed the expected increase in HR, while participants with a high HRB showed a decrease in HR. Second, the three implicit measures of main interest did not show the expected positive association with alcohol-induced HR Change. If anything, implicit arousal-associations correlated negatively with alcohol-induced HR Change. Third, participants who reported strong explicit immediate arousal expectancies (VAS) reported higher alcohol consumption (even within this sample of heavy drinkers) and reached their peak BAC faster. Late explicit sedation expectancies were found to be positively associated with the amount of alcohol-related problems. No associations were found between explicit cognitions and HR Change. Fourth, implicit approach-associations, measured with the IAT, were positively associated with attentional bias for alcohol, as measured with a blocked alcohol Stroop. However, due to the lack of reliability of the approach-IAT, no firm conclusions can be drawn from the findings involving this measure (notwithstanding the fact that reliabilities of implicit measures are usually much lower compared with explicit measures). The arousal-IAT was not correlated with attentional bias on the blocked Stroop. 
These findings have several implications. First, alcohol-induced HR acceleration during the ascending limb of the BAC curve appears to be a good indicator of the sensitivity to the stimulating effects of alcohol in men with a multi-generational $\mathrm{FH}$ of alcoholism (Conrod et al., 1997; Peterson et al., 1996) and sensation seeking individuals (Brunelle, et al., 2005). The present sample mainly consisted of men with a negative family history ( $\mathrm{FH}$-; only 2 participants had a first degree relative with alcohol dependence) and report very similar HR responses to alcohol compared with $\mathrm{FH}$ - individuals in previous studies (Brunelle et al., 2004; Conrod et al., 1997; Fromme et al., 2004). In our study, the lack of HR increase to alcohol appeared be moderated by HRB: individuals with a low HRB showed the expected alcohol-induced HR increase, while individuals with a high HRB showed an alcohol-induced decrease in HR. Hence, especially in FH-, HRB is important to consider in an alcohol administration procedure. This study further showed that the observed strong $\mathrm{HR}$ increase in $\mathrm{FH}+$ individuals (Conrod et al., 1997) is rarely observed in heavy drinking $\mathrm{FH}$ - individuals, using the exact same protocol (only 12/48 participants showed a HR increase of more than $5 \mathrm{Bpm}$ during the ascending limb). This finding suggests that alcohol-induced HR increase might not be a good general measure of a sensitized psychomotor stimulant reaction to alcohol (Robinson and Berridge, 2003), at least not in $\mathrm{FH}$ - individuals.

Rajan et al. (1998) found that HR Variability (HRV) was a more sensitive measure than mean $H R$ to register cue-reactivity in alcoholics. HRV is variation in HR influenced by both sympathetic and parasympathetic neural activity. Low HRV is indicative of defective inhibition mechanisms in the central nervous system, which could lead to dysfunctional behavior, such as alcohol problems (Ingjaldsson et al., 2003). Possibly, HRV is a more sensitive measure to reflect the psychomotor stimulant reaction than mean $\mathrm{HR}$ that was used in this study.

Second, we did not find the hypothesized positive correlation between implicit arousal-associations and HR increase after drinking. This hypothesis was based on the idea that both the implicit arousal-associations and the alcohol-induced HR increase could reflect a sensitized psychomotor stimulant reaction to alcohol, in heavy drinkers. Unexpectedly, the effect of implicit arousal-associations on alcoholinduced HR Change was even negative. Why would individuals with strong arousal-associations show a decrease in HR after drinking a high dose of alcohol? There was a tendency for participants scoring high on arousal associations to show a higher HRB to start with. Possibly, the alcohol arousal-IAT does not measure the temporal effect we suspected the IAT to measure: arousal after drinking alcohol. Note that the IAT measures associations between alcohol and arousal, which could in principle represent both relationships: from drinking alcohol to experiencing arousal and from being aroused to starting to drink alcohol (cf. Wiers and Stacy, 
2006). These findings suggest that the arousal-IAT scores might reflect arousal prior to drinking, possibly anticipatory appetitive arousal (cf. Krank et al., 2005; Palfai and Ostafin, 2003), which may be dampened by drinking in these individuals. As has been found in previous research, the Arousal-IAT correlated positively with alcohol problems.

The positive association between HRB and the arousal-IAT could also be explained as the result of a cue-reactivity effect. Alcohol cues in the IAT and the sipprime the participants received prior to the start of the IAT, might have caused an increase in resting HR before the alcohol administration procedure was started. Obviously, participants already experiencing an increase in their resting HRB would be less likely to show a strong HR increase after alcohol consumption. This might explain why we found the negative association between the arousal-IAT and alcohol-induced HR increase: a sip-prime and performing the arousal-IAT led (in some individuals) to an increase in HRB, which resulted in a less strong HR increase or a HR decrease after alcohol consumption. In this sense the negative correlation could be an artifact of the priming procedure and IAT measurement before alcohol administration (see limitations below).

Third, we found that strong explicit immediate arousal expectancies (VAS), measured before alcohol administration, were associated with a faster peak BAC and a higher alcohol consumption. Thus, an alternative interpretation is that, at least in $\mathrm{FH}$ - individuals, it might not be HR Change, but the speed with which the peak $B A C$ is reached, that reflects the amount of arousal an individual expects from alcohol. Note that in this sample, these HR and BACs were not correlated. After the alcohol administration, however, measures of subjective arousal and sedation (POMS and BAES) indicated that participants who reached their peak BAC fastest, experienced more sedative (and not stimulating!) effects. Subjective stimulation was positively associated with HR increase during the falling BAC curve and non-significantly (but in the expected direction) during the ascending curve. No associations were found between the implicit measures (arousal, approach associations and attentional bias), alcohol consumption and the Latency to peak BAC, indicating that implicit and explicit measures might show a dissociation. Some research has shown as well that explicit measures show stronger relationships with drinking measures compared with implicit measures (e.g. Kramer and Goldman, 2003). This is an additional indication that both measures tap into different underlying processes.

Fourth, the sip-primed emotional Stroop tasks (mixed and blocked) led to different results. The mixed Stroop, containing neutral, alcohol-related and color words, did not reveal a significant emotional Stroop effect (the heavy drinkers did not show interference by alcohol words relative to neutral words). This was proba- 
bly due to carry-over effects (strong interference) of the color words (cf. Waters et al., 2003). Since the color words were processed most slowly, this indicates that they grabbed the attention more than the alcohol-related words did. From our findings, it is recommended that in using a mixed Stroop, color words are not included. The blocked Stroop did reveal a significant emotional Stroop effect which correlated positively with the strength of the implicit approach associations. The same association has been found in smokers (Mogg et al., 2005). This is the first study that found a positive association between attentional bias and approach associations in heavy drinkers. Both indirect measures were not significantly correlated with implicit arousal associations, alcohol-induced HR Change, Latency to peak BAC, Alcohol Use or Problems. Thus, both attentional bias and approach associations were not related to drinking outcome measures and physiological measures, at least not in this sample. The lack of association with alcohol use and problems could be due to restriction of range, since the sample consisted of heavy drinkers only.

Several limitations to the study should be mentioned. First, the sample size of 48 participants was relatively small. Second, since the sample consisted of only heavy drinkers, effects that were there, might not have been found, due to a restriction of range and therefore too little variation. We decided not to include light drinkers in this study because the development of arousal associations and incentive sensitization is not expected in this sample. Furthermore, it was judged unethical to ask light drinkers to consume 5 shots of vodka within 5 minutes time. A third limitation might be the lack of $\mathrm{FH}+$ men. Only 2 participants indicated having a first degree family member with alcohol dependence. The effects of a strong alcohol-induced HR acceleration found by other researchers (Conrod et al., 1997; 2001 ; Peterson et al., 1996) were specific for $\mathrm{FH}+$ individuals, predisposing them to the development of an alcohol problem. Stronger effects might have been found if more $\mathrm{FH}+$ participants had been included, but this was not feasible. Possibly in $\mathrm{FH}$ - men, latency to reaching the peak BAC is important. A fourth limitation is the lack of a baseline measurement of HR prior to the start of the experiment, before the sip-prime and the performance of the implicit measures. These might have induced some form of cue-reactivity leading to an increase in HRB. If HRB would have been measured at the start of the experiment, this speculation could have been tested. This relates to the final limitation: another association might have been found for the Arousal IAT and HR Change if the implicit measures would not have been performed before alcohol administration but on a separate day. Possibly, alcohol-related stimuli that were processed during performance of the IAT and emotional Stroop task, led to a cue-reactivity effect which caused resting HRB to 
increase before the alcohol administration procedure started, leaving less room for a further HR increase after alcohol consumption.

This study was the first to combine three types of indirect measures (arousal, approach associations and attentional bias) with the psychophysiological response to a high dose of alcohol. The hypothesized positive association between implicit arousal associations and alcohol-induced HR Change could not be confirmed. Taken into account the small sample size and the usually small effect sizes in this type of research, the lack of a positive association could be due to a lack of power, but finding a negative correlation between arousal associations and HR Change suggests that there is a small chance that implicit arousal associations would in fact be positively correlated to alcohol-induced HR Change in $\mathrm{FH}$ - heavy drinking males. Taken together, one can conclude that in heavy drinkers, implicit arousalassociations, HRB, explicit arousal and sedation expectancies and a short latency to peak BAC all seem to be intertwined with each other, predisposing an individual to the development of a possible alcohol problem. The strength of explicit arousal expectancies heavy drinkers have of the immediate effects of a high dose of alcohol seems to be influenced more by the speed at which the peak BAC is reached than by the amount of $\mathrm{HR}$ acceleration. Further research, including $\mathrm{FH}+$ participants, is needed to unravel the complex associations between explicit and implicit alcohol cognitions on the one hand and how they relate to psychophysiological responses to alcohol on the other hand.

\section{AUTHOR NOTE}

Esther van den Wildenberg, Margreet Beckers, Femke van Lambaart and Reinout Wiers, Department of Experimental Psychology, Maastricht University, Maastricht, the Netherlands. Patricia Conrod, National Addiction Centre, Institute of Psychiatry, King's College London, UK.

This research was supported in part by a 'VIDI' grant from the Dutch National Science Foundation (N.W.O.) to RWW (452.02.005).

\section{ACKNOWLEDGEMENTS}

The authors wish to thank Jan de Houwer and Gerty Lensvelt-Mulders for their useful suggestions concerning the statistical analyses and Katrijn Houben for programming the computer tasks. 


\section{Appendix: IAT stimuli}

\section{Target stimuli:}

Alcohol: wine, beer, whisky, port, vodka and rum.

Soda:, Cassis, Sinas (both lemonades), Spa (sparkling water), Coke, tonic, and juice.

\section{Valence Attribute stimuli:}

Pleasant: love, sunshine, warmth, peace, hug, rainbow Neutral: paper, circle, ballpoint, factory, truck, magnet Unpleasant: sorrow, war, depression, pain, fight, disease. Neutral: letter, square, page, machine, scissors, window

\section{Arousal/Sedation Attribute stimuli:}

Active: talkative, jovial, restless, alert, unrestrained, rambunctious

Neutral: constant, wide, brown, digital, recent, historic

Quiet: silent, listless, sleepy, passive, relaxed, calm.

Neutral: oval, compact, related, central, extensive, steep

Irrelevant control block materials:

Materials: rubber, plastic, aluminium, linen, metal, marble Neutral: usual, curved, joined, always, completely, angular

Approach-avoidance Attribute stimuli:

Approach: Toward, to touch, to grasp, to grab, to approach, ahead Avoidance: From, to run away, to escape, to leave, to flee, departure 



\section{CHAPTER 3}

\section{DO IMPLICIT ALCOHOL AROUSAL AND APPROACH ASSOCIATIONS CORRELATE WITH ATTENTIONAL BIAS IN ALCOHOL- DEPENDENT PATIENTS?}

Submitted as: Van den Wildenberg, E., Coolen, W., Wiideveld, A. G. M., Meulders, W., \& Wiers, R. W. Do Implicit Alcohol Arousal and Approach Associations Correlate with Attentional Bias in Alcohol-Dependent Patients? 


\section{ABSTRACT}

Aims: An attentional bias for alcohol-related stimuli and automatic alcohol-related memory associations with both arousal and approach have all been hypothesized to reflect a sensitized appetitive response in heavy drinkers. This study was designed to investigate the associations between the three constructs in alcohol-dependent patients and controls.

Methods: Sixty-seven alcohol-dependent patients and 21 controls performed six short Implicit Association Tests and two emotional Stroop tasks. After performing the implicit tests, craving, alcohol expectancies and alcohol-related problems were assessed.

Results: Unexpectedly, the groups did not differ on the unipolar arousal-IAT. Only the bipolar approach-avoidance IAT could differentiate between the groups, with patients being less avoidant than controls. A significant attentional bias on the Stroop was found only for patients with a relatively short-term alcohol problem. Their blocked Stroop-effect correlated .40 with the arousal-IAT. Explicit measures, such as expectancies and craving, were found to be more strongly associated with alcohol measures than the implicit measures.

Conclusions: The attentional bias finding in the short-term alcoholic patients could be interpreted as support for the incentive habit theory of addiction rather than the incentive sensitization theory.

Keywords: Alcohol Stroop; Alcohol-IAT; Incentive-Sensitization; Incentive-Habit; Alcoholism. 


\section{INTRODUCTION}

Addiction or substance dependence is a form of psychopathology in which other than rational processes play an important role. The addict generally knows that the (mis) use of the drug has many negative consequences such as health problems, divorce and financial problems and that this destructive behavior should be brought to a halt. In spite of this knowledge the addicted individual continues to compulsively look for the drug in order to use it. Several automatic or implicit processes are involved in the transition from 'normal' to compulsive use that exert their influence on behavior. Implicit Cognition will be used as an "umbrella term" for attentional bias and implicit memory associations (Wiers \& Stacy, 2006).

One important automatic process that might be involved in the development and maintenance of addictive behaviors and other forms of psychopathology (e.g. anxiety disorders) is attentional bias (Cox, Fadardi, \& Pothos, 2006; Harvey, Watkins, Mansell, \& Shafran, 2004). The individual's attention is drawn to stimuli related to his/her current concerns (Klinger \& Cox, 2004; see below) which can guide subsequent behavior. Attentional bias can be measured with various reaction time tasks. A widely used task to assess attentional bias is the addiction-Stroop task (for a review see Cox et al., 2006). In this task the participant is required to respond to the color of the word while ignoring the contents. Emotionally relevant words grab the attention, which interferes with the color naming response, leading to slower reaction times. In many studies, alcohol-dependent patients have been found to respond more slowly to alcohol-related words compared with neutral words (Cox et al., 2006).

A second automatic process thought to be involved in addiction is the development of implicit memory associations. These associations are formed by past drinking experiences which can be influenced by the neurochemical reactions to alcohol (also genetically determined; Wiers \& Stacy, 2006). One way of measuring implicit alcohol-related memory associations is by means of the Implicit Association Test (IAT; Greenwald, McGhee, \& Schwartz, 1998). The IAT is a computerized categorization task consisting of 5 phases in which the participant is asked to categorize words either belonging to a target category (e.g. 'Alcohol' versus 'Soda') or an attribute category (e.g. 'Arousal' versus 'Sedation') by means of two response keys. In one combination phase the target 'Alcohol' shares the same response key as the attribute 'Arousal'. In the reversed combination phase, the position of the attribute switches thereby placing 'Alcohol' and 'Sedation' under the same response key (see Table 3.1). The difference in reaction times between these two combination phases is called the IAT-effect (see Wiers, van de Luitgaarden, van den Wildenberg, \& Smulders, 2005; Wiers, van Woerden, Smulders, \& de Jong, 


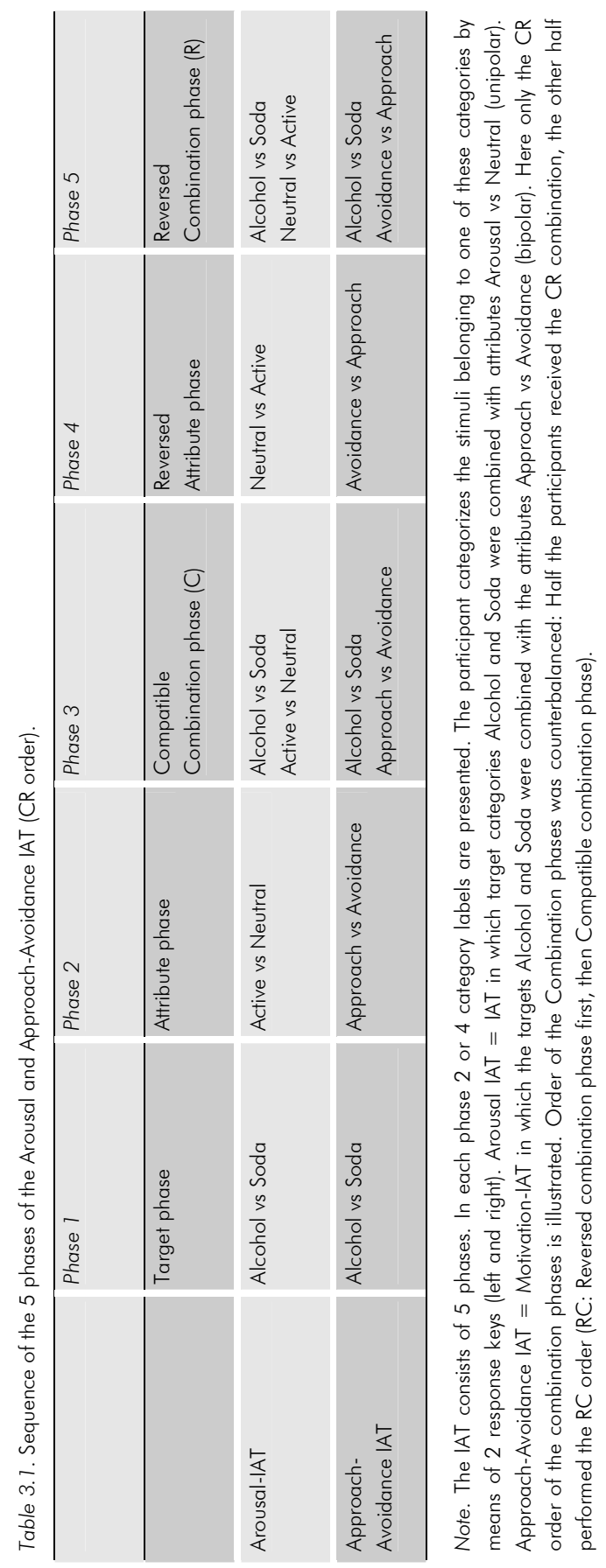


2002 for a more detailed description). The logic underlying the IAT is that reaction times are relatively fast when two concepts that are strongly related in memory, share the same response key (Greenwald et al., 1998). IAT research in the addiction field has shown that heavy drinkers associate alcohol more strongly with arousal than with sedation on the Arousal-IAT, which was not the case for light drinkers (Wiers et al., 2002). Associations between alcohol and arousal have also been found in alcohol-dependent patients (De Houwer, Crombez, Koster, \& De Beul, 2004). One issue in using the IAT to assess alcohol-associations is that both the target category (i.e. alcohol versus soda) and the attributes (i.e. arousal versus sedation) are bipolar. Therefore, the alcohol-arousal associations found in heavy drinkers could (partly) represent soda-sedation associations. This possibility was recently investigated by using unipolar versions of the IAT (Houben \& Wiers, 2006). It was found that heavy drinkers have associations with arousal and sedation, but that the arousal-associations were stronger. On the Valence-IAT (positive versus negative) both light and heavy drinkers associated alcohol more strongly with negative valence than with positive valence, both on a bipolar IAT (Wiers et al., 2002) and on unipolar IATs (Houben \& Wiers, 2006; Van den Wildenberg, Beckers, van Lambaart, Conrod, \& Wiers, 2006). Palfai and Ostafin (2003) showed with their Motivation-IAT that alcohol-approach associations were positively related to binge drinking and to craving for alcohol and subjective arousal in a cue exposure experiment.

There are several reasons, both biological and cognitive, why we expect that measures of attentional bias and implicit memory associations might be related to each other. First, it has been hypothesized that attentional bias for alcohol-related stimuli and alcohol approach and arousal -associations all reflect sensitization of the brain reward system after repeated alcohol use (Franken, 2003; Palfai \& Ostafin, 2003; Wiers et al., 2002, respectively). According to the Incentive-Sensitization Theory of Robinson and Berridge $(1993 ; 2003)$ drugs cause structural changes in brain areas that are normally involved in natural reward and motivation (e.g. in evolutionary advantageous processes such as searching for food). These neuroadaptations make the system hypersensitive to drugs and drug-related stimuli: the (initial) effect of the drug becomes stronger after repeated use, due to a heightened dopamine release in the nucleus accumbens. This psychomotor stimulant reaction might be translated into implicit arousal and approach associations with the drug. Incentive sensitization has also been linked to attentional bias for the drug (Franken, 2003; Robinson \& Berridge, 2003). The sensitized brain reward system mediates the process of 'incentive salience' or "wanting" of the drug and not the pleasurable component of drug use, called "liking". Because the system can oper- 
ate in absence of conscious awareness, the addict may not understand why the urge to use is so strong while the "liking" component has diminished.

Besides this hypothesized neurobiological background for the relationship between implicit alcohol associations and attentional bias, several cognitive frameworks have been proposed to integrate memory and attentional processes. The general cognitive theory by Logan (2002) proposes that cognition is a single phenomenon and that attention and memory share one underlying process, but are tested differently. In other words, they may represent two sides of the same coin. According to the theory of current concerns (Klinger \& Cox, 2004), substance use, over time, becomes a nonconscious, automatized goal. A current concern is directed at pursuing this goal. Throughout the goal-pursuit of substance use, the current concern remains active and will consciously or unconsciously bias cognitive processing towards this goal. In competition for the focus of attention with other current concerns, the addiction-related concern will 'win' because of the disproportionate value it has obtained.

The goal of the present study was to investigate the associations between implicit alcohol arousal and approach associations (IAT) with attentional bias for alcohol-related stimuli (emotional Stroop), within a group of alcohol-dependent patients. It was hypothesized that arousal-associations, approach-associations and $A B$ correlated positively in the patient group. In addition, a control group of addiction clinic employees (familiar with alcohol-related words and stimuli; Ryan, 2002) was tested to investigate if these implicit cognitions only appeared in alcoholdependent patients. We expected that the patients would display a stronger $A B$ on the Stroop and stronger arousal and approach associations on the IAT compared with controls.

In addition, we were interested in the relationships between implicit and explicit measures. We hypothesized that alcohol measures (e.g. age of onset, alcohol use and problems), explicit alcohol arousal expectancies and self-report craving would correlate positively with each other and with the three implicit measures. Finally, we wanted to investigate whether alcohol-dependent patients with and without a family history of alcoholism would differ with regard to their implicit cognitions and explicit arousal expectancies. This was based on the finding of Wiers and colleagues (2000) that family history positives (FHPs) reported stronger positive alcohol expectancies for a high dose compared with family history negatives (FHNs). This could be due to their different genetic make-up resulting in a stronger response to the rewarding effects of alcohol (e.g. Gianoulakis, Krishnan, \& Thavundayil, 1996). In a non-clinical sample (data from Wiers et al., 2005, but not presented due to space limitations) we indeed found stronger implicit alcohol arousal associations in FHPs than in FHNs $(p<.05$, one-tailed). 


\section{MATERIALS AND METHODS}

\section{Participants}

Sixty-seven alcohol-dependent patients were recruited (50 men) with a mean age of 45.30 years $(S D=9.31)$. Furthermore, a group of 21 addiction clinic employees (7 men) with a mean age of 37.29 (SD = 12.36) were tested. Recruitment took place at different alcohol-rehabilitation clinics in the region (Limburg) belonging to either the "Mondriaan Zorggroep" (N=41) or the "Verslavingszorgcircuit Noord en Midden Limburg" ( $N=26$ ). Patients were included when they met the criteria for alcohol dependence according to the DSM IV and they were required to be sober at the day of testing. Patients were excluded when they were dependent on another drug than alcohol or cigarettes and when they were suffering from another severe psychiatric disorder (e.g. schizophrenia), as became clear during the intake session by their physician.

\section{Procedure}

The study was approved of by the Ethics Committee of the Academic Hospital in Maastricht. Participants were tested at the clinic where they were first informed orally as well as in writing, about what would happen during the test session of approximately $90 \mathrm{~min}$. After the participants signed the informed consent, they were asked to perform the IAT and Stroop tasks (in a counter-balanced order). Subsequently, they were interviewed about their alcohol consumption (EuropASI) and Family History of alcoholism. Finally, they were asked to fill out the questionnaires on alcohol expectancies, craving and alcohol problems.

\section{Measures}

\section{Alcohol Measures}

Patients were asked what they usually drank before treatment on an average day. Answers such as "20 half a liters of beer" were afterwards recoded into standard alcoholic drinks according to the coding table of the Composite International Diagnostic Interview (CIDI; Robins et al., 1989). Furthermore, they were asked how many days they were abstinent at the time of testing. They were interviewed about their alcohol and drug use with Section III of the EuropASI (Kokkevi \& Hartgers, 1995) in which they were asked for the age of onset at which 'more than 5 glasses per occasion at least 3 times per week' were consumed. This was used as an indication of the start of problematic drinking behavior. Furthermore, it was calculated how many years in total their drinking had been problematic. In addition to the 
EuropASI interview, controls filled out a quantity-frequency questionnaire (based upon Sobell \& Sobell, 1992) from which weekly alcohol consumption was calculated. A final alcohol measure was the 10-item Alcohol Use Disorder Identification Test (AUDIT, Saunders, Aasland, Babor, de la Fuente, \& Grant, 1993), measuring alcohol use (items 1-3) and problems (items 4-10). Mean AUDIT score of the patients was $23.16(S D=8.10)$ versus $4.38(S D=2.40)$ for the controls. A cut-off of 10 is used as an indication of alcohol problems. Alcohol consumption per day before treatment ranged between 4 to 134 standard drinks with an average of 24.66 per day ( $S D=21.38$ ). Days of abstinence before testing ranged from 0 to 1460 , with an average of 104.52 days (SD $=233.83$ ). Controls were light drinkers with a weekly consumption ranging between 0 and 28 standard drinks with an average of 7.05 (SD $=7.47)$. The mean number of years they were working in the addiction health care was 7.81 years $(S D=6.98)$.

\section{Family History of Alcoholism}

$\mathrm{FH}$ was determined after interviewing the participants about all relatives that might have (had) a possible alcohol problem (based on the Familiy History Tree by Mann, Sobell, Sobell, \& Pavan, 1985). A participant was categorized as FHP when at least one first-degree family member suffered from alcohol dependence. 64\% were classified as $\operatorname{FHN}(N=43), 33 \%$ as $\operatorname{FHP}(N=22)$, and of 2 patients data was missing. The controls were predominantly FHNs ( $N=18 ; 86 \%)$.

\section{Explicit Alcohol Expectancies}

Explicit Alcohol Expectancies were assessed with the Dutch Alcohol Expectancy Questionnaire (Wiers, Hoogeveen, Sergeant, \& Gunning, 1997). Only the items of positive and negative expectancies for a high dose of alcohol were used. The High Dose Positive subscale consisted of 11 items (e.g. "After drinking a lot of alcohol I feel self-assured"). This positive expectancy subscale contained a smaller subscale of 5 items that reflected Tension Reduction expectancies for a high dose (e.g. "I enjoy drinking when I'm bored"). The High Dose Negative subscale consisted of 9 items (e.g. "After drinking a lot of alcohol I feel down"). All items were answered on a 5-point Likert scale ranging from "I don't agree" to "I absolutely agree".

\section{Craving}

Craving was measured with the Dutch version of the Obsessive Compulsive Drinking Scale (Schippers et al., 1997) on which participants indicated their (obsessive) thoughts and feelings about alcohol in the past 7 days. Mean OCDS score for the patient group was $15.18(S D=11.84)$ versus $4.44(S D=5.33)$ for the controls. A 
cut-off of 7 is used to distinguish between social drinkers and dependent individuals (Anton, 2000).

\section{Emotional Stroop task}

The Stroop task consisted of four blocks and was presented on a laptop. In the first block 40 practice trials were given, consisting of ten different non-semantic strings such as \#\#\#\#. Every stimulus was practiced once in each of the four colors: red, yellow, green and blue. The participant was required to respond with one of four differently colored response keys, on a separate response box. The second block was a quasi random presentation of three stimulus word categories: neutral, alcohol-related and color (total of 96 trials). The neutral category consisted of 8 semantically related words belonging to the category 'traffic' (e.g. 'car'). The alcohol-related category also consisted of 8 stimulus words (e.g. 'pub'). Stimuli from the neutral and alcohol-related categories were matched on number of syllables. Each stimulus word of both categories was presented once in every color. Further, the four color words were added to the random block. Each of the four color words was presented twice in every color. The presentation of a stimulus word in the random block was never followed by a stimulus from the same word category. A oneminute break was given after the random block which was followed by the third and fourth block in which the neutral respectively alcohol-related stimuli were presented in a blocked fashion. In each block all 8 stimuli were presented once in every color. Each stimulus remained on the screen until the participant responded, with a maximum of 3 seconds. If the participant was too slow (>3000 ms) the message 'faster' was presented. In case of an incorrect response the feedback 'wrong' appeared on the screen. The intertrial-interval was 2 seconds.

\section{Implicit Association Test}

Six short IATs were presented. Modified versions of the IAT were used in which the associations of alcohol and soda with Positive, Negative (valence dimension), Arousal and Sedation attributes (arousal dimension) were tested in a unipolar way, versus the contrast attribute category 'Neutral' (cf. Houben \& Wiers, in press; Van den Wildenberg et al., 2006). These 4 IATs were partly counterbalanced with a Latin Square. Approach-avoidance attributes were measured in a bipolar fashion and were always presented as the fifth IAT. To control for possible artifacts of the IAT measurement, a unipolar control-IAT 'Materials' was included as the sixth IAT.

Labels used for the target categories were 'Alcohol' and 'Soda'. Labels used for the 5 unipolar attribute categories were: 'Pleasant' (positive), 'Unpleasant' (negative), 'Active' (arousal), 'Quiet' (sedation) and 'Materials' (control), all versus 
the contrast category 'Neutral'. The bipolar IAT carried the labels 'To Approach' versus 'To Avoid'.

Both target and attribute categories consisted of 6 stimuli each. The two Valence IATs used nouns that were matched for word length, familiarity and number of syllables and were neutral, on average, with respect to arousal. In the Arousal and Sedation IATs adjectives were used, also matched for word length, familiarity and number of syllables and were neutral, on average, with respect to valence. Stimuli in the Approach-Avoidance IAT were matched on word length. Stimuli in the control-IAT were matched on familiarity, valence and arousal. All stimuli can be found in the Appendix.

The first IAT consisted of 5 phases (see Table 3.1). In phase 1, the target categories 'Alcohol' and 'Soda' were practiced. This phase was practiced once, as the first phase of the first IAT. The next IATs consisted of 4 phases. In phase 2 the attribute categories (e.g. 'Active' vs 'Neutral') were introduced. In phase 3, the first combination phase $(C)$, the target and attribute categories were combined. In phase 4 the attribute categories were reversed (e.g. 'Neutral' vs 'Active') and in the final phase, the reversed combination phase $(R)$, the targets and reversed attributes were combined again. In the single phases (1, 2 and 4) either the 6 target or 6 attribute stimuli were presented twice leading to a total of 24 trials per phase. In the two combination phases ( 3 and 5 ) all target and attribute stimuli were presented twice as well leading to a total of 48 trials. Order of the two combination phases was counterbalanced, with half the participants always starting with the easier Combination phase (CR) and the other half starting with the Reversed Combination phase (RC).

Stimulus words were presented in black in the middle of the screen against a light grey background. Each stimulus word remained on the screen until a response was generated, with a maximum of $3 \mathrm{sec}$. When a participant was too fast ( $<300$ $\mathrm{ms}$ ) or too slow (>3000 ms) the messages 'too fast' or 'too slow' appeared on the screen. In case of an incorrect response the feedback 'wrong' was presented in red letters under the stimulus word. The intertrial interval was 250 milliseconds. All IAT's were programmed in ERTS 3.18 (Beringer, 1996). Reliabilities of the IATs were calculated by the Spearman-Brown corrected correlation between the practice and test trials of the combination phases. The internal consistencies were as follows: Positive: .86, Negative: .85, Arousal: .71, Sedation: .81, ApproachAvoidance: .57 and Control: .64 . 


\section{RESULTS}

First, findings regarding the implicit measures and explicit expectancies comparing alcohol-dependent patients and controls will be presented. Second, correlations between the three implicit measures within the patient sample are described, covering the main hypothesis. Third, correlations between the explicit, implicit and alcohol measures are presented. Fourth, an explorative analysis on attentional bias and the length of the alcohol problem is expounded. Finally, FH effects were examined.

\section{Implicit Alcohol-related Cognitions}

\section{Emotional Stroop task}

Overall error rate for all the trials was $2.62 \%$, with no significant differences between the groups $(t(86)=-1.03, p=.31)$. In the analyses only correct logtransformed response times were used. A main effect of condition was found, such that patients responded more slowly to all word categories of both Stroop tasks compared with controls $(F(81)=2.49, p<.05$; Figure 3.1). For controls as well as patients both emotional Stroop-effects (alcohol-neutral words) were nonsignificant (Table 3.2). Both random and blocked Stroop-effects (alcohol-neutral words) in the patient group were in the expected direction, with the alcohol words being processed somewhat slower, but non-significantly. The classic color Stroopeffect (incongruent - congruent color words) was found to be significant in both groups, indicating that the incongruent color words were being processed more slowly than the congruent words.

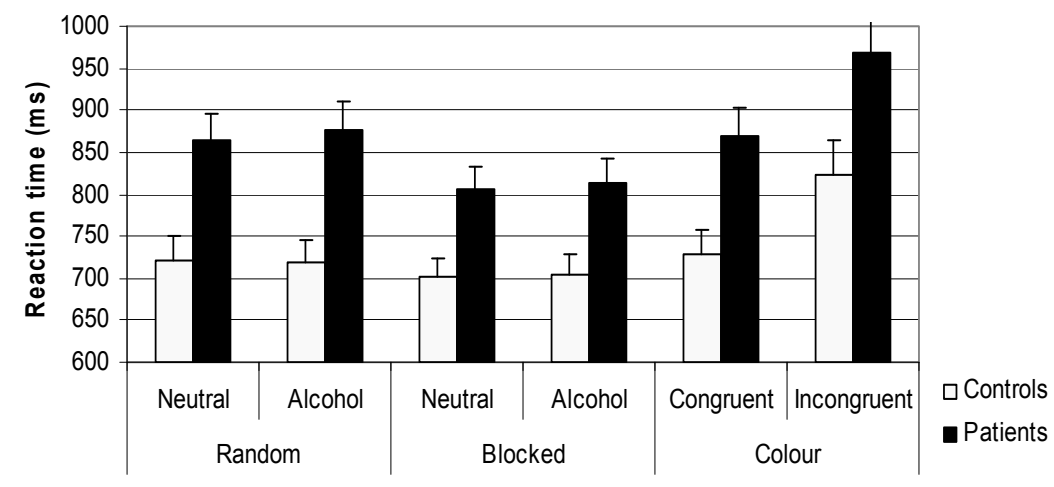

Word categories per Stroop

Figure 3. 1. Reaction times per word category for the Random Stroop (neutral, alcohol, color) and Blocked Stroop (neutral, alcohol) are presented, both for the controls and alcohol-dependent patients. For each bar the standard error is provided. 
Implicit Association Test

The conventional IAT-effects in milliseconds (Greenwald et al., 1998) are presented in Table 3.2. IAT-effects are difference scores: if responses are faster in the compatible $C$ phase than the incompatible $R$ phase, the IAT-effect is positive. Analyses used the new D600 algorithm (Greenwald, Nosek, \& Banaji, 2003). The largest effect sizes were found for the Negative, Arousal and Control IATs. The percentage of errors over all 6 IATs was significantly higher for the patients (7.5\%) compared with the controls $(5.0 \% ;+(68.34)=3.20, p<.01)$.

\section{Multivariate Effect of Emotional Stroop and IAT}

To prevent Type I errors due to multiple testing, a 2 (Group) x 2 (Gender) MANOVA was run on the two Stroops, the Arousal and the Approach-Avoidance IAT, with age as covariate. We expected these 4 measures to form one underlying dimension on which the groups would differ. The interaction between group $x$ gender was non-significant $(p>45)$ and was therefore deleted from the model. The final MANOVA showed a borderline significant multivariate effect of group, $F(4$, $82)=2.46, p=.052$. Non-significant effects were found for gender $(p=.09)$ and the covariate age $(p>35)$. The relative contribution of the 4 implicit measures to the effect of group was determined with discriminant analysis with structure coefficients (Huberty \& Morris, 1989): Approach-Avoidance IAT $=.86$, Blocked Stroop $=.35$, Random Stroop $=.24$, Arousal-IAT $=-.07$. Thus, the ApproachAvoidance IAT contributed the most to the multivariate difference between patients and controls and the Arousal-IAT the least. A 'control' MANOVA was run on the measures for which no differences between the groups were expected (classicStroop, positive, negative, sedation and control IAT). No significant main or interaction effects were found for group, age or gender ( $p s>$.15).

\section{Explicit Alcohol Expectancies}

Independent t-tests revealed that patients reported significantly stronger positive $(t(84)=5.18, p<0.001)$ and tension reduction expectancies $(+(54.73)=8.76, p$ $<0.001$ ) compared with controls. No differences between the groups were found on negative expectancies $(t(84)=1.08, p=0.28)$. 


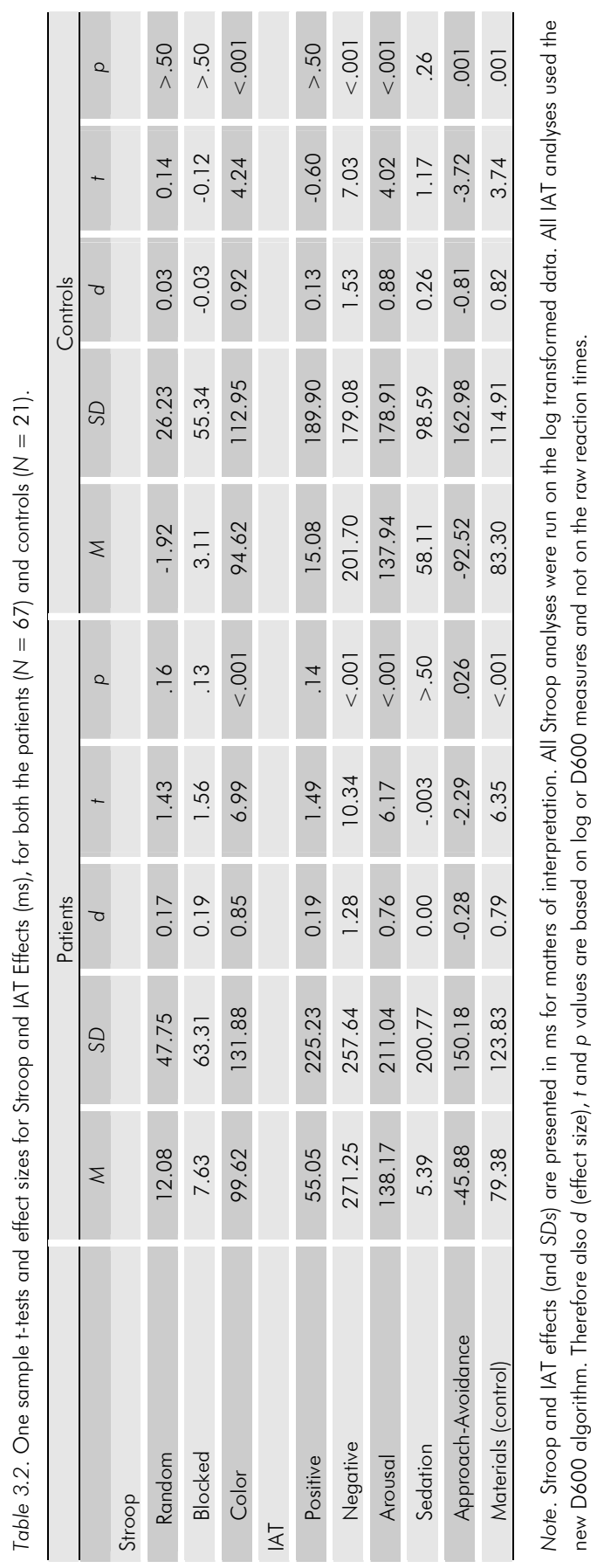




\section{Correlation Analyses within the Patient-group}

Correlations between Implicit Measures

All correlations between implicit, explicit and alcohol measures with $p$-values below 0.30 are presented in Table 3.3. Our main focus was on the correlations between the implicit arousal and approach associations and attentional bias. The correlations with the arousal and approach IAT were controlled for the materials-IAT in order to reduce measurement artifacts (the pattern of results was similar to the correlations without the control-IAT). The hypothesized positive correlation between arousal and approach associations was not found $(r=.04, p>.50)$, nor was there an association between the approach-IAT and the Stroop tasks ( $p s>.20$ ). There was a significant positive correlation of .26 $(p<.05)$ between the arousalIAT and the blocked Stroop-effect.

\section{Correlations between Explicit, Implicit and Alcohol Measures}

The explicit measures (expectancies and craving) hardly correlated with the implicit measures (Table 3.3). A significant negative correlation of $-.29(p<.05)$ was found between the blocked Stroop and negative expectancies for a high dose, indicating that patients with a strong attentional bias had fewer negative alcohol outcome expectancies. Furthermore, the approach-IAT correlated positively with alcohol problems measured with the AUDIT $(r=.30, p<.05)$. None of the implicit measures was associated with craving on the OCDS.

Craving was associated with higher alcohol consumption $(r=.35, p<.01)$, more alcohol-related problems (AUDIT, $r=.29, p<.05$ ), and stronger explicit positive expectancies for a high dose $(r=.48, p<.01)$. Also, an early age of onset of the alcohol problem was associated with more alcohol use $(r=-.48, p<$ $.01)$ and problems $(r=-.26, p<.05)$, more craving $(r=-.31, p<.05)$ and stronger explicit positive $(r=-.28, p<.05)$ as well as negative expectancies $(r=-$ $.26, p<.05)$ for a high dose. 


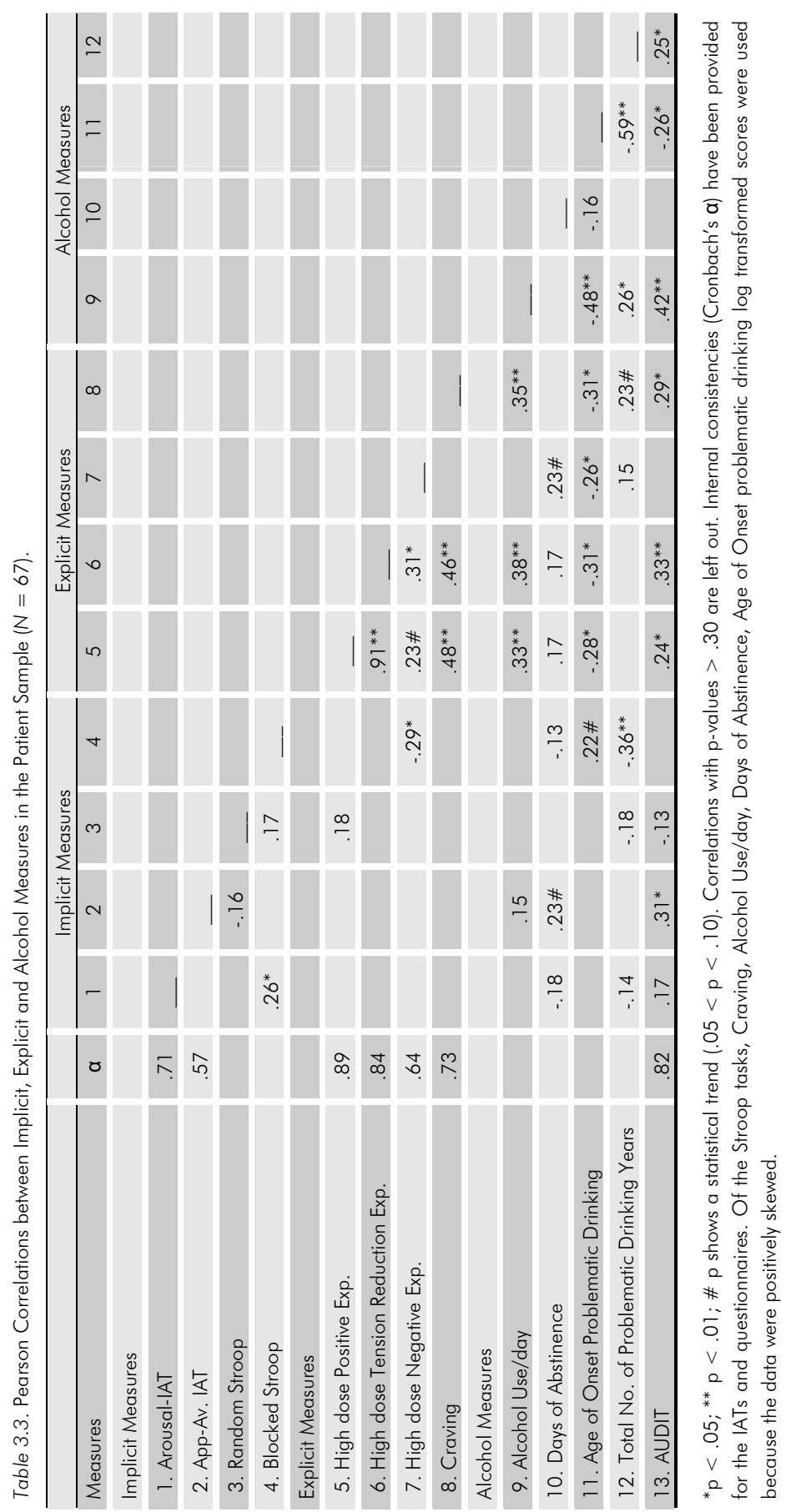




\section{Attentional Bias}

A negative correlation of $-.36(p<.01)$ was found between $A B$ on the blocked Stroop and the years of problem-drinking (Table 3.3). Thus, as the duration of the alcohol problem was longer, $A B$ decreased. We decided, on an exploratory basis, to perform a median split upon the total number of problematic drinking years (16). A 3 (Group: controls, ST and LT alcoholics) x 2 (Gender) MANOVA with both Stroops as dependent measures, revealed a significant main effect of problematic drinking years $(p<.05)$. Only short-term (ST) alcoholics revealed a significant $A B$, both on the random $(+(33)=2.07, p<.05 .047)$ and blocked $(t(33)=3.37, p<$ .01) Stroop (Figure 3.2). The blocked Stroop-effect of the ST alcoholics differed significantly from the controls $(t(53)=2.20, p<.05)$ and from the LT alcoholics $(t(65)=3.25, p<.01)$. In this subgroup of ST-patients, the correlation between the arousal-IAT and the blocked Stroop was .40 ( $p<.05)$. No significant correlation between the arousal-IAT and blocked Stroop was found for the long-term (LT) patients $(r=.13, p=.50)$.

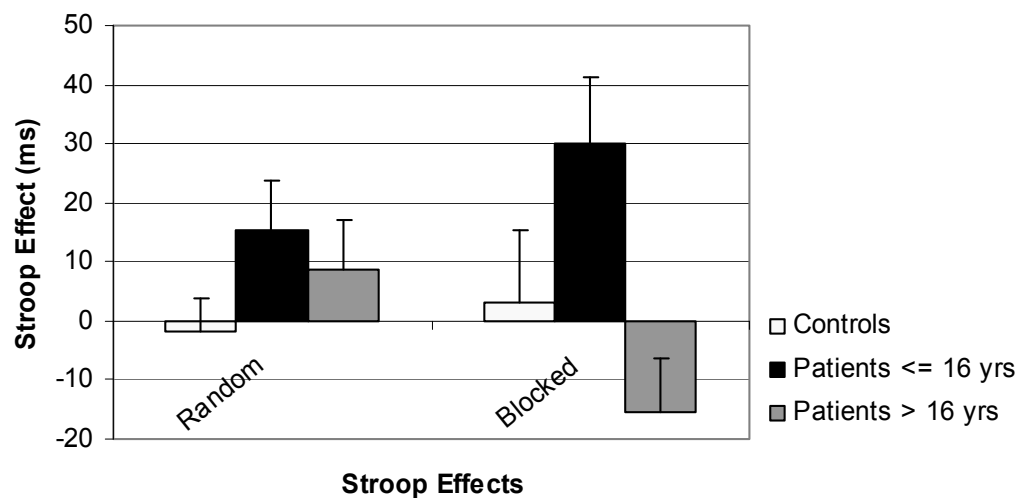

Figure 3.2. Stroop-effects are presented for the Random and Blocked Stroop. Based on a median split upon the total number of problematic drinking years, the subgroup of patients with the relatively short duration of the problem ( $\leq 16$ years) shows a significant attentional bias on both Stroop tasks. The controls and patients with a longer duration of the problem ( $>16$ years) show no significant attentional bias on either of the tasks. For each bar the standard error is provided.

\section{Influence of $\mathrm{FH}$}

No significant differences were found on any of the implicit, explicit or alcohol measures between alcohol-dependent patients with and without a $\mathrm{FH}$ of alcoholism (ps > .12). 


\section{DISCUSSION}

A first finding of this study was that alcoholic patients and controls familiar with alcohol problems differed at borderline significance on the four tests of implicit processes in addiction assessed here. This multivariate effect was primarily due to patients showing weaker associations between alcohol and avoidance than controls. The other measures (alcohol Stroops and arousal-IAT) did not differentiate well between the two groups. Main objective of this study was to investigate the relationship between implicit alcohol-related memory associations and attentional bias in alcoholic patients. Overall, no strong associations were found. Only the blocked Stroop and arousal IAT correlated significantly. There was an indication that attentional bias was stronger in relatively short-term alcoholics than in longterm alcoholics. In this subgroup of patients, also the strongest correlation between the blocked Stroop and the arousal IAT was found. On the IAT, both patients and controls displayed mainly negative and arousal associations. Finally, while the explicit measures (craving and expectancies) correlated significantly with several alcohol measures (and were able to differentiate between the groups), this was hardly the case for the implicit measures. Also, correlations between implicit and explicit measures were virtually absent.

These findings have several implications. First, the lack of strong associations between the implicit measures could be due to several reasons. On the one hand, methodological limitations due to the counterbalancing of the implicit measures (done to prevent learning effects from affecting the same IATs) could have increased the amount of error in this correlational design. Also, the design of the Stroop might not have been fully optimal (see Cox et al., 2006). More robust effects might have been found if a voice key were used instead of response keys (Cox et al., 2006). Other measures such as the visual probe task might have been more sensitive in measuring attentional bias. The Stroop-effect could be described as an interference effect. It might be an ambiguous measure of attentional bias since no information about the components of attention (initial shift versus maintenance) is obtained (Field, Mogg, \& Bradley, 2006). On the other hand, it is possible that the three measures do not tap into the same underlying mechanism we expected it to do. Only the positive correlation between arousal-associations and attentional bias is suggestive of a partly similar underlying process which is measured with the arousal-IAT and the emotional Stroop. This association was the strongest in the STpatients.

However, the finding that both patients and controls performed similarly on the arousal-IAT (both IAT-effects were $138 \mathrm{~ms}$ ) makes it questionable whether the arousal-IAT used here measures the process of incentive-sensitization we suspected 
it to do. The controls were light drinkers, not expected to be sensitized due to the effects of long-lasting alcohol use. It should be noted that this study made use of a unipolar arousal-IAT, instead of the bipolar version that was used in the Wiers et al. (2002) study where a difference between light and heavy drinkers was found on the arousal-IAT. Additionally, significant IAT-effects were found in both groups on the control-IAT ('materials'). This is another indication that the unipolar IAT might not measure what we expect it to measure. Another explanation for the lack of a discriminative effect of the arousal-IAT used here, might be the type of word stimuli that was used. In the study by Wiers et al. (2002) only 'positive arousal' words were used such as "cheerful". In the present study the arousal words were neutral with respect to valence, therefore both positive arousal words (e.g. "talkative") as well as negative arousal words (e.g. "restless") were used. Possibly, it is mainly the positive arousal words that reflect the arousal-associations we want to measure. Contrary to earlier findings with the unipolar arousal-IAT in students (Houben \& Wiers, 2006; Van den Wildenberg, Beckers, van Lambaart, Conrod, \& Wiers, 2006), in this study, no association was found between the arousal-IAT and alcohol problems. Therefore, taken together, this version of the IAT might lack construct validity.

Second, the finding that the only IAT that correlated with alcohol-problems and was able to differentiate between the groups was the bipolar approachavoidance IAT, might be indicative of a better construct validity of the bipolar IAT compared with the unipolar IAT. Due to the relativity of the bipolar approachavoidance IAT, this finding can be explained in several ways. Possibly, the controls were more avoidant because they work as addiction clinic employees and have seen the devastating effects of alcohol abuse. Another explanation could be that the patients are less avoidant because they have stronger approach-associations which 'compensate' their avoidance-associations. Possibly, patients were ambivalent in their approach-avoidance associations. This could have been influenced by the context they were in at the time of testing: all patients were receiving treatment and were tested at the clinic. This might have induced stronger alcohol-avoidance associations. Future IAT research is recommended to further investigate the validity of the unipolar versus the bipolar IAT.

Third, the finding that only ST-patients displayed a significant attentional bias for alcohol could be interpreted in favor of the incentive-habit theory (Di Chiara, 2000), rather than the incentive-sensitization theory (Robinson \& Berridge, 1993) of addiction. According to the incentive-sensitization theory, attentional bias should increase as the length of the alcohol problem increases and the brain becomes more and more sensitized (thus predicting the strongest attentional bias in the LTpatients). Conversely, the incentive-habit theory would predict a decrease in attentional bias on the long-term (after the initial incentive sensitization 'phase'), due to 
structural changes in the brain which, in the end, result in a diminished incentive value of the drug. The brain automatically reacts to conditioned drug-cues, independently of the incentive value obtained in the initial phase of the addiction. Indeed, no significant attentional bias was found for the subgroup of LT-patients. Also, in the group of LT-patients, no significant association between the arousal IAT and the blocked Stroop was found. To our knowledge, this is the first study with alcohol-dependent patients that provided some support for the incentive-habit theory. In a study with smokers, it was found that light smokers showed a stronger attentional bias for smoking-related cues compared with heavy smokers (Mogg, Field, \& Bradley, 2005).

Fourth, the stronger findings with the blocked Stroop than the random Stroop might be explained by presence of rumination or carry-over effects in the random Stroop (Waters, Sayette, \& Wertz, 2003). The response to the stimuli can be influenced by the word preceding the target word. Since the color words were the slowest to be processed, this may have affected the response to the alcohol and neutral words. The random Stroop usually yields smaller effects than the blocked Stroop (Cox et al., 2006). The finding that the alcohol Stroop effects are relatively slow and even evident on the next trial, have been interpreted as evidence that relatively slow components in attention (disengagement) are particularly important in addictive behaviours (Cox et al., 2006; Field et al., 2006).

Fifth, as has been reported more often, very few and low correlations were found between the implicit and explicit measures (Hofmann, Gawronski, Gschwendner, Le, \& Schmitt, 2005). This is again an indication that explicit and implicit measures, at least partly, tap into different underlying constructs. The explicit measures in this study showed stronger correlations with the alcohol measures than the implicit measures did. This might either suggest methodological flaws in the use of for example the unipolar IAT or it might be an indication that the explicit measures do in fact show stronger associations with drinking measures and drinking behavior. It is also evident from these findings and from other alcohol-IAT findings, that implicit measures should be viewed as a potentially useful additional tool to, rather than as a replacement of, explicit measures.

Besides the above mentioned limitations to the study with respect to some of the methodological issues regarding the IAT and Stroop tasks, it should be mentioned that an additional limitation to the study is the suboptimal control group. Due to differences with regard to sample size, age and gender both groups were not matched well, leading to a loss of power and comparison difficulties.

Taken together, the expected pattern of findings with the implicit measures receives mixed support based on the incentive-sensitization theory (no differences between patients and controls on implicit arousal-associations and $A B$ ), but pa- 
tients did show less strong automatic avoidance reactions. Only in the ST alcoholic patients, a significant alcohol-related $A B$ was found which correlated with arousalassociations. This could be interpreted as support for the incentive-habit theory of addiction.

\section{ACKNOWLEDGEMENTS}

The authors would like to acknowledge all the physicians and addiction clinic employees for the recruitment of the patients at the different clinics. Furthermore, we would like to thank the research assistants Femke van Lambaart and Tim Satijn for collecting part of the data. This research was partly funded by ZONMW (grant 31000065); RWW is funded by a 'VIDI' grant from the Dutch National Science Foundation (N.W.O., grant 45202005). 


\section{Appendix: IAT stimuli}

\section{Target stimuli}

Alcohol: wine, beer, whisky, port, vodka and rum.

Soda:, Cassis, Sinas (both lemonades), Spa (sparkling water), Coke, tonic, and juice.

\section{Valence Attribute stimuli:}

Pleasant: love, sunshine, warmth, peace, hug, rainbow Neutral: paper, circle, ballpoint, factory, truck, magnet Unpleasant: sorrow, war, depression, pain, fight, disease. Neutral: letter, square, page, machine, scissors, window

Arousal/Sedation Attribute stimuli:

Active: talkative, jovial, restless, alert, unrestrained, rambunctious Neutral: constant, wide, brown, digital, recent, historic Quiet: silent, listless, sleepy, passive, relaxed, calm.

Neutral: oval, compact, related, central, extensive, steep

Irrelevant control IAT materials:

Materials: rubber, plastic, aluminium, linen, metal, marble Neutral: usual, curved, joined, always, completely, angular

Approach-avoidance Attribute stimuli:

Approach: Toward, to touch, to grasp, to grab, to approach, ahead Avoidance: From, to run away, to escape, to leave, to flee, departure 



\section{CHAPTER 4}

\section{PREDICTING TREATMENT RESPONSE OF ALCOHOL-DEPENDENT PATIENTS RECEIVING NALTREXONE WITH IMPLICIT AND EXPLICIT ALCOHOL-RELATED COGNITIONS}

Submitted as: Van den Wildenberg, E., Wiideveld, A. G. M., Verhaegh, H., Meulders, W., \& Wiers, R. W. Predicting Treatment Response of AlcoholDependent Patients Receiving Naltrexone, with Implicit and Explicit Alcohol-Related Cognitions. 


\section{ABSTRACT}

Aims: Many alcohol-dependent patients relapse during or after treatment. Goal of this study was to predict treatment outcome using implicit and explicit measures. Methods: Nineteen alcohol-dependent patients were treated with the mu-opioid antagonist naltrexone for three months in the period 2003-2004. Three outcome variables were of main interest: relapse (t-test), time to relapse (Cox regression) and percentage of drinking days (correlation). They were predicted with family history of alcoholism (FH), explicit measures (craving, alcohol expectancies and motivation) and implicit measures (the emotional alcohol Stroop and alcohol arousal and approach associations, measured with the Implicit Association Test).

Results: Patients who relapsed tended to show stronger implicit alcohol avoidance associations on the IAT, were less distracted by alcohol-related words on the Stroop and were predominantly Family History Positives (FHPs) compared with the patients who remained abstinent. Craving at treatment admission did not predict relapse. More exploratively it was investigated whether changes occurred in the explicit and implicit measures over the course of the first six weeks of treatment.

Conclusions: Although the study suffered from recruitment difficulties, even in this small sample differences were found between patients who relapsed and those who did not. Implicit measures may have an additional value in the prediction of relapse in alcohol-dependent patients.

Key words: Alcohol dependence; naltrexone; treatment outcome; predictors; family history of alcoholism; implicit alcohol associations; attentional bias; craving; alcohol expectancies; motivation 


\section{INTRODUCTION}

Alcoholism continues to affect a substantial part of the population each year. Many patients seek treatment for their alcohol problem at some point in their lives. Some patients are successful at remaining abstinent, but approximately half of the alcohol or other drug-dependent patients returns to active substance use within a year after treatment has ended (McLellan et al., 2000). It would be beneficial if we were able to predict who would be at a greater risk to relapse. This way, treatment strategies such as relapse prevention (Marlatt and Gordon, 1985) and pharmacotherapy can be directed at these particular individuals who might need it the most.

It is well established that individuals with alcohol problems are more easily distracted by alcohol-related stimuli than individuals who drink less (Cox et al., 2006; Field et al., 2006). There is evidence that in alcohol misuse and dependence, alcohol-related stimuli capture the attention of the heavy drinker, i.e. it is especially hard to disengage attention from the alcohol stimulus. This might subsequently induce craving and heighten the risk of relapse. One measure to assess attentional distraction by alcohol-related stimuli is the emotional Stoop task (for a review, see Cox et al., 2006). In this color-naming task the participant is required to ignore the contents of the word while responding only to the color the word is printed in. For example if the word "blue" is printed in yellow, one responds to yellow. In case of an emotionally relevant stimulus word (e.g. "beer" for an alcohol abuser), the default word reading response interferes more strongly with the less common colornaming response which results in slower reaction times (for color in all subjects, for alcohol stimuli only in heavy drinkers or alcoholics). A study by Cox et al. (2002) used the emotional Stroop task to predict treatment outcome (relapse or dropout) in 23 alcohol-dependent patients. The task included stimuli that were either neutral (string of symbols), alcohol-related (personalized, favorite brand names), or words related to a personal, current concern (Klinger and Cox, 2004). The results showed that of the 14 treatment completers, the 9 alcohol abusers who relapsed displayed a significant increase in interference by the alcohol-related words four weeks later at the end of treatment. This was not the case for controls and for the five patients who had remained abstinent. Additionally, the nine patients who dropped out of treatment showed higher interference scores at treatment admission for the stimuli of current concerns (e.g. problems related to relationships, finances or health), compared with the controls and treatment completers. These findings suggest that two important processes play a role. First, patients who become more preoccupied with alcohol during treatment are at a higher risk to relapse. Second, patients who start treatment while concerned about other important aspects in their lives seem to be less motivated to focus on their alcohol problem and therefore end treatment 
early. In a smoking cessation study, similar results were found; more attentional distraction by smoking-related words was associated with a lapse in the short-term (Waters et al., 2003). More recently, attentional bias measured with the emotional Stroop was found to predict relapse in a sample of heroin-dependent patients (Marissen et al., 2006).

Besides attentional bias, two other relatively automatic processes have been proposed that might play a role in problematic drinking behavior: implicit alcohol arousal and approach associations. These are more general automatically activated memory associations. Both types of associations can be measured with the Implicit Association Test (IAT; Greenwald et al., 1998). The IAT is a computerized categorization task consisting of 5 phases (see Table 4.1). In phase one, the participant categorizes stimuli belonging to a target category (e.g. alcohol versus soda) with a left and right response key. In the second phase, stimuli of the attribute phase (e.g. arousal versus sedation) are categorized. The third phase is the first combination phase in which target and attribute categories are presented together (e.g. press left for alcohol + arousal words and press right for soda + sedation words). In the fourth phase the position of the target words switches and in the fifth phase, the reversed combination phase, the targets and attributes are again combined (e.g. press left for soda + arousal and left for alcohol + sedation). The difference between both combination phases is called the IAT-effect. The idea underlying the IAT is that when two concepts that are more strongly related in memory share the same response key, reaction times will be faster (Greenwald et al., 1998). Several studies have found that implicit alcohol arousal associations correlated positively with alcohol use or problems (Houben and Wiers, 2006; Van den Wildenberg et al., 2006; Wiers et al., 2002). Furthermore, the heavier drinkers associated alcohol more strongly with arousal than the lighter drinkers did (Wiers et al., 2002). Also, implicit alcohol approach associations have been found to be associated with binge drinking and subjective arousal reactivity after cue exposure to an alcoholic beverage (Ostafin and Palfai, 2006; Palfai and Ostafin, 2003). In a small sample of alcohol-dependent patients in treatment $(n=23)$ implicit arousal and approach associations were able to predict relapse and dropout (Wiers, Stassen, and Lemmens, in preparation). 


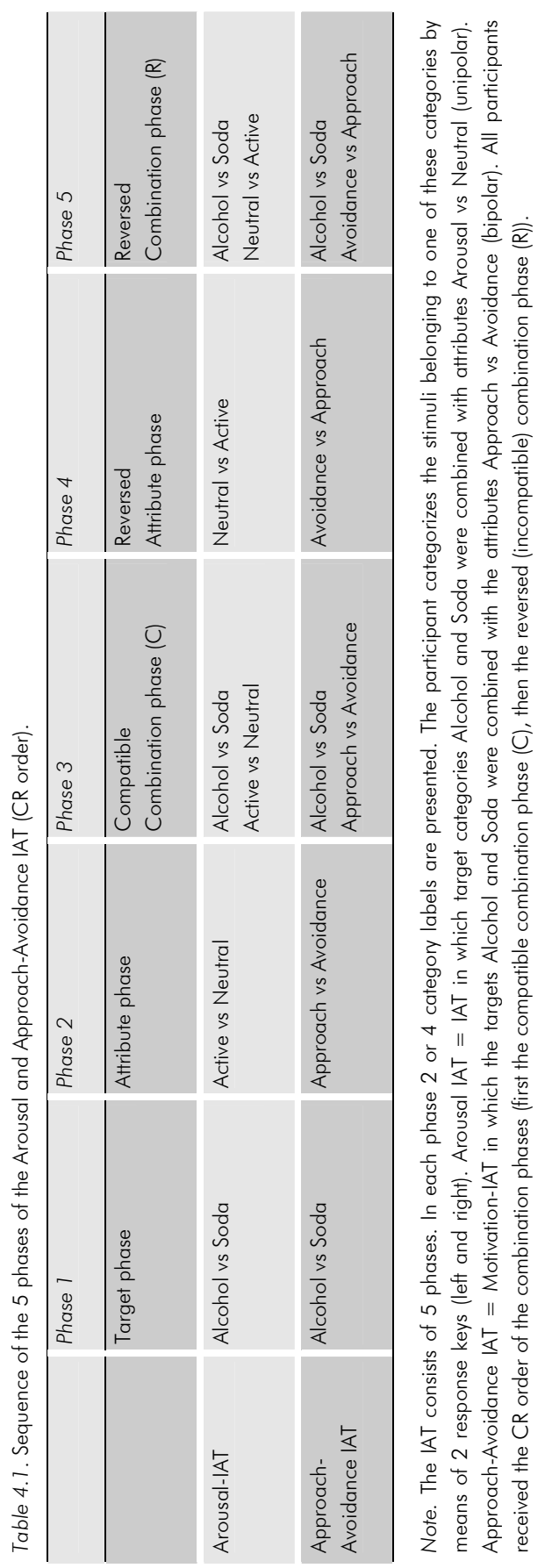


Implicit measures have shown to be able to predict additional variance in drinking behavior, above the variance explained by explicit measures such as explicit outcome expectancies (e.g. Stacy, 1997; Wiers et al., 2002). In the present study, we wanted to predict treatment outcome of 19 alcohol-dependent patients with three explicit measures (craving, alcohol expectancies and motivation) and three implicit measures (attentional bias, arousal and approach associations). All patients were receiving the opioid receptor antagonist naltrexone. Naltrexone has proven to reduce craving (O'Brien et al., 1996) and the subjective high after alcohol consumption (Volpicelli et al., 1995) by blocking the mu-opioid receptor, one of the important receptors involved in the effects of alcohol and drugs. The alcohol-induced beta-endorphin response has been found to be strongly heritable (Froehlich et al., 2000). Studies by Gianoulakis and colleagues (1996a; 1996b) showed that plasma levels of individuals with a positive family history of alcoholism (FHP) displayed lower basal activity of beta-endorphin and a stronger betaendorphin release in response to alcohol, compared with family history negatives (FHN). This suggests an enhanced sensitivity to the effects of alcohol in the FHPs. Cognitively, this could be represented as stronger alcohol arousal expectancies (Wiers et al., 1998; 2000) or as stronger automatic appetitive responses (arousal and approach associations; Wiers, in press). Therefore, it might be expected that mainly individuals with a FHP benefit from treatment with naltrexone. A clinical trial by Monterosso et al. (2001) supported this hypothesis by showing that patients who reported more craving at treatment admission and a stronger familial loading of alcohol problems reported less heavy drinking days during naltrexone treatment.

Our hypotheses in the present study were that at treatment admission FHPs would show stronger implicit alcohol arousal and approach associations (both measured with the IAT) and more attentional bias for alcohol-related words (measured with the emotional Stroop) than FHNs. Furthermore, it was expected that they would report more craving for alcohol and stronger explicit arousal expectancies and at this point. No differences between the groups were expected with regard to motivation to quit drinking. Additionally, we were interested in the variables that would predict relapse, time to relapse and percentage of drinking days during the naltrexone treatment. It was hypothesized that FHPs would benefit more from naltrexone, resulting in lower relapse rates compared with FHNs. We further explored whether changes in the cognitive implicit and explicit motivational variables over the first 6 weeks were related to treatment success. If a decrease in implicit arousal and approach associations, attentional bias, explicit arousal expectancies and craving would be found over time we expected this to be positively related to treatment outcome (abstinence). 


\section{METHODS}

\section{Participants}

Nineteen alcohol-dependent patients were recruited at different alcoholrehabilitation clinics in Limburg belonging to either the "Mondriaan Zorggroep" or the "Verslavingszorgcircuit Noord -en Midden Limburg" where they were receiving 'standard' care (e.g. weighing the pros and cons of drinking behavior, cognitive behavioral therapy and social skills training). Patients were included when they met the DSM-IV criteria for alcohol dependence, were 18 to 65 years old and were recently detoxified (a minimum of 4 days). Exclusion criteria included having such a medical history that participation was not possible (as judged by their physician), dependence to another drug than alcohol according to the DSM-IV (excluding nicotine), suffering from another axis I disorder requiring treatment (excluding the use of antidepressants), cannabis use of more than 5 days per week, the use of opiates during the past 30 days, the use of other medication against addiction (disulfiram or acamprosate), laboratory evidence of significant hepatocellular failure (ASAT and ALAT more than 2 times the norm), participation in another clinical trial during the past 30 days and for women pregnancy or breast feeding. The sample was predominantly male (79\%), from Dutch origin (95\%), had a partner (53\%) and was unemployed (74\%). Their mean age was 44.63 years (SD $=8.66$ ). Before treatment the average alcohol consumption per day was 21.37 standard alcoholic drinks (SD = 13.60). The sample was a subset of participants described in another study on different implicit measures in alcoholism. Only in the present study, treatment outcome is predicted.

\section{Materials and Measures}

\section{Alcohol Measures}

Patients were interviewed about their daily alcohol consumption before treatment with the Composite International Diagnostic Interview (CIDI; Robins et al., 1989). Further, they were asked how many days they were abstinent at the time of testing. Additionally, they were interviewed about their alcohol and drug use with Section III of the EuropASI (Kokkevi and Hartgers, 1995), in which it was assessed what their age of onset was at which 'more than 5 glasses per occasion at least 3 times per week' were consumed. This was used as an indication of the start of problematic drinking behavior. Based on the age of onset of problematic drinking and total number of years of abstinence, it was calculated how many years in total they had been drinking problematically. Another alcohol measure was the 10-item Alcohol 
Use Disorder Identification Test (AUDIT, Saunders, Aasland, Babor, de la Fuente, and Grant, 1993), measuring alcohol use (items 1-3) and problems (items 4-10). Mean AUDIT score was 24.61 (SD = 6.30). A cut-off of 10 is used as an indication of alcohol problems. A final alcohol measure was an alcohol diary, kept during the naltrexone treatment, in which all participants were asked to fill out how much they were craving for alcohol every day (scale 0-10), what were specific situations that induced this urge to drink and if they had been drinking, how much this was. Based on the alcohol diary, variables such as time to relapse, number of drinking days and drinks per drinking day could be calculated.

\section{Family History for Alcoholism}

$\mathrm{FH}$ was determined after interviewing the participants about all relatives that might have (had) a possible alcohol problem (based on the Familiy History Tree by Mann, Sobell, Sobell, and Pavan, 1985). A participant was categorized as FHP when at least one first-degree family member suffered from alcohol dependence. Of the 19 participants, 9 were categorized as FHN, and 10 were FHP.

\section{Explicit Measures}

Craving. Craving was measured with the Dutch version of the Obsessive Compulsive Drinking Scale (OCDS; Schippers et al., 1997) on which participants indicated their (obsessive) thoughts and feelings about alcohol in the past 7 days. Mean OCDS score (based on the 14-item version) was 15.94 (SD = 12.29). A cut-off of 7 is used to distinguish between social drinkers and dependent individuals (Anton, 2000). Reliability of the OCDS was found to be .94 .

Explicit Alcohol Expectancies. Explicit Alcohol Expectancies were assessed with a questionnaire consisting of 14 unipolar visual analogue scales (VAS) ranging from 0-110 mm (cf. Wiers et al., 2002; 2005). The participant was asked how much they agreed or disagreed with the statement "Drinking alcohol makes me ....". Seven items were arousal expectancies (e.g. "energetic"). The other 7 VAS items consisted of sedation expectancies (e.g. "relaxed"). Reliabilities were good with .88 for the arousal subscale and .91 for the sedation subscale.

Motivation. To assess motivation to change drinking behavior, the Alcohol Intentions Questionnaire (AIQ; Blanchard, 1999) was administered. Two items were used: desire to quit drinking and expected success for change. Both items were answered on an 11 -point Likert scale, ranging from 0 (no desire to quit or lowest success expectation) to 10 (greatest desire to quit or greatest success expectation). 


\section{Implicit Measures}

Emotional Stroop task. The Stroop task consisted of four blocks and was presented on a laptop (for a description of the exact task see Van den Wildenberg et al., 2006). The first block consisted of practice trials and the second block was a mixed Stroop presentation. Here, only blocks 3 and 4 were of interest (to obtain a blocked Stroop measure). The third block was a 'neutral' block, consisting of 8 semantically related neutral words belonging to the category 'traffic' (e.g. 'car'). The fourth block, or 'alcohol' block, consisted of 8 alcohol-related stimulus words (e.g. 'pub'). Stimuli from the neutral and alcohol-related categories were matched on the number of syllables. In each block all 8 stimuli were presented once in every color (red, green, yellow, blue). The participant was required to respond with one of the four colored response keys on a separate response box. Each stimulus remained on the screen until the participant responded, with a maximum of $3 \mathrm{sec}$ onds. If the participant was too slow (>3000 ms) the message 'faster' was presented. In case of an incorrect response the feedback 'wrong' appeared on the screen. The intertrial-interval was 2 seconds. The emotional Stroop effect was obtained by subtracting the neutral words from the alcohol-related words (alcoholneutral). Thus, a positive Stroop effect indicates distraction by alcohol-related words.

Implicit Association Test. Six short IATs were presented (for further details see Van den Wildenberg et al., 2006). Of particular interest were the arousal-IAT and approach-avoidance IAT. A modified version of the arousal-IAT was used in which the associations of alcohol and soda with arousal were tested in a unipolar way, versus the contrast attribute category 'neutral' (cf. Houben and Wiers, 2006). The Arousal-IAT was partly counterbalanced within a Latin Square with 3 other IATs (Positive, Negative and Sedation, which are not of main interest here). The approach-avoidance attributes were measured in a bipolar fashion and were always presented as the fifth IAT. Target categories of both IATs were labeled 'Alcohol' and 'Soda'. The label used for the arousal attribute category was 'Active'. The bipolar IAT carried the labels 'To approach' versus 'To avoid'. Both target and attribute categories consisted of 6 stimuli each. In the Arousal-IAT adjectives were used, matched for word length, familiarity and number of syllables. Furthermore, they were, on average, neutral with respect to valence. Stimuli in the ApproachAvoidance IAT were matched on word length. All target and attribute stimuli words are shown in the appendix.

During performance of the IATs, the category labels were presented in the left and right upper corners of the computer screen and remained there during the entire task. Stimulus words were presented in black in the middle of the screen 
against a light grey background. Each stimulus word remained on the screen until a response was generated, with a maximum of $3 \mathrm{sec}$. When a participant was too fast ( $<300 \mathrm{~ms}$ ) or too slow ( $>3 \mathrm{~s}$ ) the messages 'too fast' or 'too slow' appeared on the screen. In case of an incorrect response the feedback 'wrong' was presented in red letters under the stimulus word. The intertrial interval was 250 milliseconds. All IAT's were programmed in ERTS 3.18 (Beringer, 1996).

The IAT consisted of 5 phases (see Table 4.1). In phase 1, the target categories 'Alcohol' and 'Soda' were practiced. The target phase was practiced only once, as the first phase of the first IAT. The next IATs consisted of 4 phases. In the second phase the attribute categories (e.g. 'Active' vs 'Neutral') were introduced. In the third phase, the first combination phase $(\mathrm{C})$, the target and attribute categories were combined. In the fourth phase the attribute categories were reversed (e.g. 'Neutral' vs 'Active') and in the final phase, the reversed combination phase (R), the targets and reversed attributes were combined again. In the single phases (1, 2 and 4) either the 6 target or 6 attribute stimuli were presented twice leading to a total of 24 trials. In the two combination phases ( 3 and 5) all target and attribute stimuli were presented twice as well leading to a total of 48 trials. Order of the two combination phases was not counterbalanced. Since we were interested in the prediction of treatment outcome with measures of individual differences, this variable was kept constant (cf. Asendorpf et al., 2002). All participants started with the Combination phase (CR order), which usually yields the strongest IAT-effects. The IAT-effect is the subtraction of the $C$ phase from the $R$ phase $(R-C)$, thus, a positive Arousal IAT effect is indicative of stronger associations of alcohol with arousal than with neutral words (unipolar measurement). A positive Approach-avoidance IAT effect is indicative of stronger associations of alcohol with approach than with avoidance words (bipolar measurement). Reliabilities of the IATs were calculated by the Spearman-Brown corrected correlation between the practice and test trials of the combination blocks. The internal consistencies were .63 for the Arousal-IAT and .84 for the Approach-Avoidance IAT.

\section{Procedure}

The study was approved of by the Medical Ethics Committees of Venlo and Maastricht. All participants provided written informed consent, after they were informed about the study orally (by their physician) as well as in writing. During the first visit the patients were asked to perform the emotional Stroop and IATs (counterbalanced). Subsequently, they were interviewed about their alcohol consumption, alcohol-related problems (CIDI and EuropASI) and FH for alcoholism. Finally, they were asked to fill out the craving (OCDS), expectancy (VAS) and motivation (AIQ) questionnaires. On the subsequent day, each patient started the naltrexone treat- 
ment. All patients received an oral dosage of $50 \mathrm{mg}$ naltrexone (ReVia ${ }^{\circledR}$, Dupont Pharma) per day, for a period of 12 weeks. In order to enhance motivation and compliance, all patients received additional support with the so-called BRENDA technique describing 6 steps: Biopsychosocial evaluation, Report, Empathy, Needs, Direct Advice and Assessment (Volpicelli et al., 2001). During the 12 weeks of treatment, 5 visits were planned (1, 3, 6, 9 and 12 weeks after inclusion). Topics such as general well-being, medication compliance, the alcohol diary and high risk situations that had been encountered were discussed during the 30 minute visits. To investigate possible changes during treatment, a second measurement of implicit arousal and approach associations, attentional bias, explicit expectancies, craving and motivation took place at the fourth visit (6 weeks after inclusion).

Medication came in pill boxes containing as many capsules as there were days in between the visits with an additional 6 to prevent non-compliance in case a visit was rescheduled. At each visit the number of capsules brought back was counted. This way, a fairly reliable indication of treatment compliance can be obtained (Farmer, 1999). The 12 week naltrexone treatment period was followed by a 12 week medication-free period.

\section{Outcome variables}

Due to dropout during the 6 months of the study, data of only 11 patients were available for the follow-up. Therefore, it was decided to perform analyses only on the 12-week naltrexone treatment period. Three main outcome variables were used in the analyses: 1) Relapse (defined as the presence of at least one heavy drinking day, with heavy drinking day defined as 6 or more drinks per occasion for men and 5 or more for women); 2) Time to relapse (defined as number of days from the start of the naltrexone treatment till the first heavy drinking day); and 3) Percentage of drinking days (defined as the number of days on which at least one alcoholic drink was consumed divided by the number of days for which data was available). Our goal was to predict Relapse, Time to relapse and Percentage of drinking days with explicit measures (craving, alcohol expectancies and motivation) and implicit measures (arousal-IAT, approach-IAT and emotional Stroop). FH, age, days of abstinence before treatment and duration of the alcohol problem were used as covariates because all were, to a certain extent, correlated with relapse (see Table 4.2). 


\section{Statistical Analysis}

Due to a lack of power, a full logistic regression, including covariates, on the dichotomous variable relapse was not possible. Therefore, separate t-tests were performed on the main continuous variables (predictors) of interest, testing the difference between relapsers and non-relapsers (Hosmer and Lemeshow, 1989). Chisquare tests were used to assess the effects of $\mathrm{FH}$ and gender on relapse. Time to relapse was analyzed with Cox regression (backward stepwise, Likelihood Ratio) and percentage of drinking days was subjected to correlational analysis (multiple regression was not possible due to the small sample size and the relatively large number of variables that was associated with this outcome variable). The Cox regression analysis was controlled for the covariates $\mathrm{FH}$, age, days of abstinence before treatment and duration of the alcohol problem. Only in case of a significant contribution of the covariate, it was kept in the analysis. First each variable (covariate, implicit or explicit measure) was entered separately. Variables with $p$-values < .50 were all entered in a 2 -step backward regression analysis. Covariates were entered at step 1 and the remaining variables at step 2 . In order to prevent masking effects, the final backward model which resulted from this analysis was subsequently extended by entering each of the variables that was removed from the model during the backward regression separately at step 2 . All variables with a $p$ value $<.30$ when added separately were entered simultaneously in the final backward regression model. Next, all variables with p-values $>.30$ were removed, resulting in the final model. Correlations between all measures (3 outcome variables, covariates, explicit and implicit measures) are presented in Table 4.2.

\section{RESULTS}

First, the results of FHPs versus FHNs at treatment admission will be reported. Next, variables that predicted the 3 outcome measures (relapse, time to relapse and percentage of drinking days) are described. Finally, more exploratively, it is investtigated whether changes in implicit and explicit cognitions occurred over the course of the first 6 weeks of treatment.

\section{Family History effects}

At treatment admission, no differences were found between FHPs and FHNs with regard to their implicit arousal associations, implicit approach associations, attentional bias or explicit arousal expectancies ( $p s>.40$ ). Analysis of the OCDS craving scores revealed that, before naltrexone treatment, FHPs were craving significantly more than FHNs, $t(16)=-2.25, p<.05$. Furthermore, on the AIQ, FHP's 
tended to have less desire to quit, $+(6.79)=2.16, p=.069$ and they had a lower success expectation of achieving their desired goal, $t(16)=3.00, p<.01$. Additionally, FHPs tended to report more alcohol-related problems as revealed by a higher AUDIT score, $t(16)=-2.09, p=.053$, and they were less days abstinent before treatment (41 days for FHNs versus 21 for FHPs), $+(15.81)=2.96, p<$ 01 .

\section{Treatment outcome measures}

\section{Medication Compliance, Adverse Events and Dropout}

Compliance was defined as the intake of at least $80 \%$ of the naltrexone capsules (Pettinati et al., 2000) during participation in the trial and was based on pill count as registered in the case report form. One patient (who relapsed but did not dropout) was non-compliant. Of three other patients who relapsed or dropped out, compliance could not be determined. Several adverse events were reported, mainly during the first weeks of the treatment: nausea (42\%), sleep problems (26\%), headache $(26 \%)$, dizziness $(21 \%)$, fatigue $(26 \%)$, decreased appetite (16\%), sweating (16\%), dry mouth (11\%), irritability (11\%), rash (5\%), decreased libido (5\%), weight gain (5\%), constipation (5\%) and tingling feelings (5\%). Total dropout rate was $32 \%$ during the first 3 months of naltrexone treatment ( 6 out of 19) and $42 \%$ after 6 months (8 out of 19). Interestingly, patients who dropped out tended to report a higher expectation of success in achieving their drinking goal than patients who remained in treatment, $r(18)=.46, p=.057$, which could suggest some form of overestimation or socially desirable answering in this subgroup.

\section{Relapse}

As can be seen in Table 4.3 several significant and marginally significant differences were found between patients who relapsed and who maintained abstinent during treatment. A strong association was found between a FHP and the risk of relapse: of the 7 patients who relapsed, 6 were FHP, versus 4 out of 12 in the nonrelapse group $(p<.05)$. Furthermore, the patients who relapsed tended to be older $(p=.09)$, tended to have a longer total duration of the alcohol problem ( $p$ 


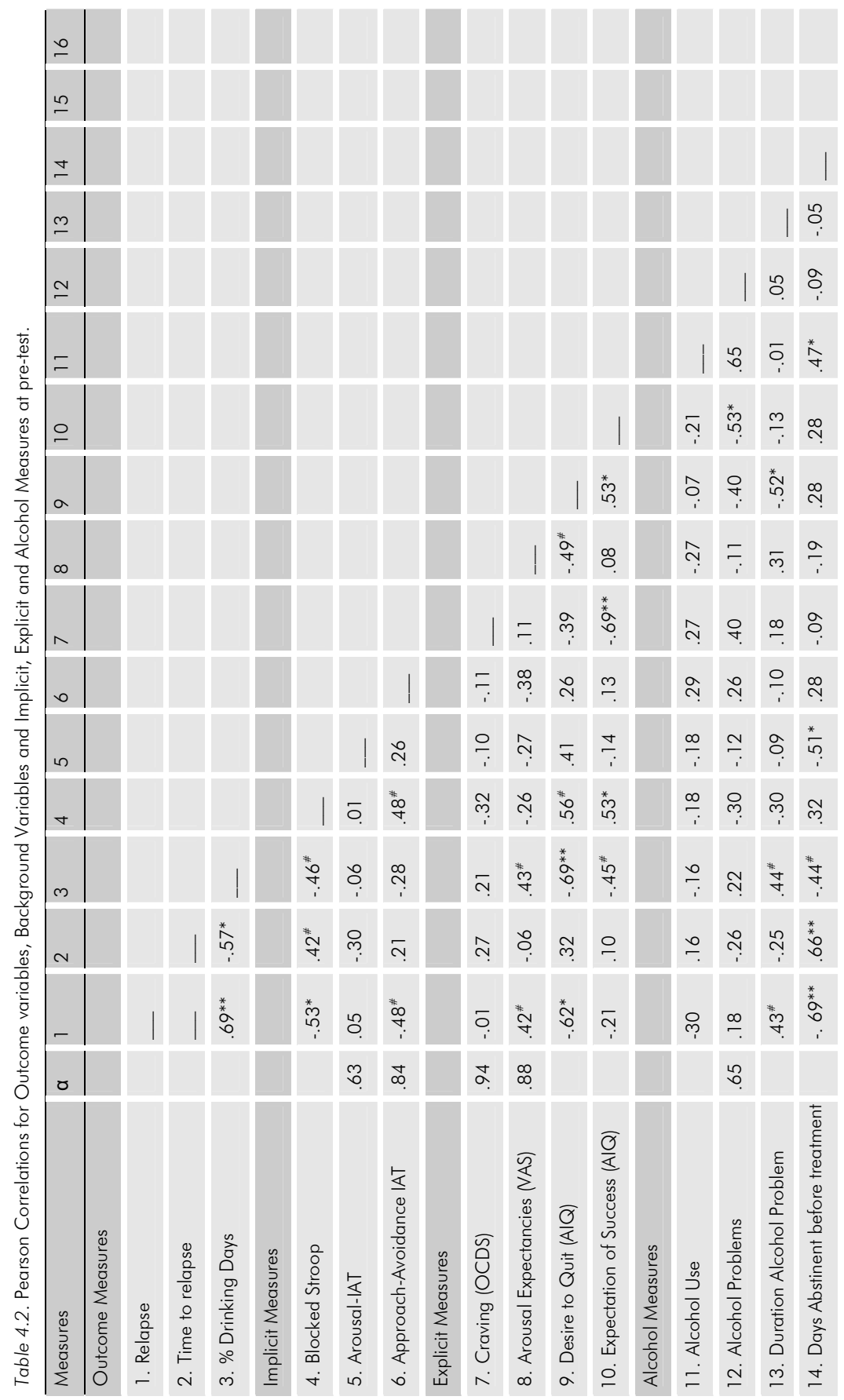




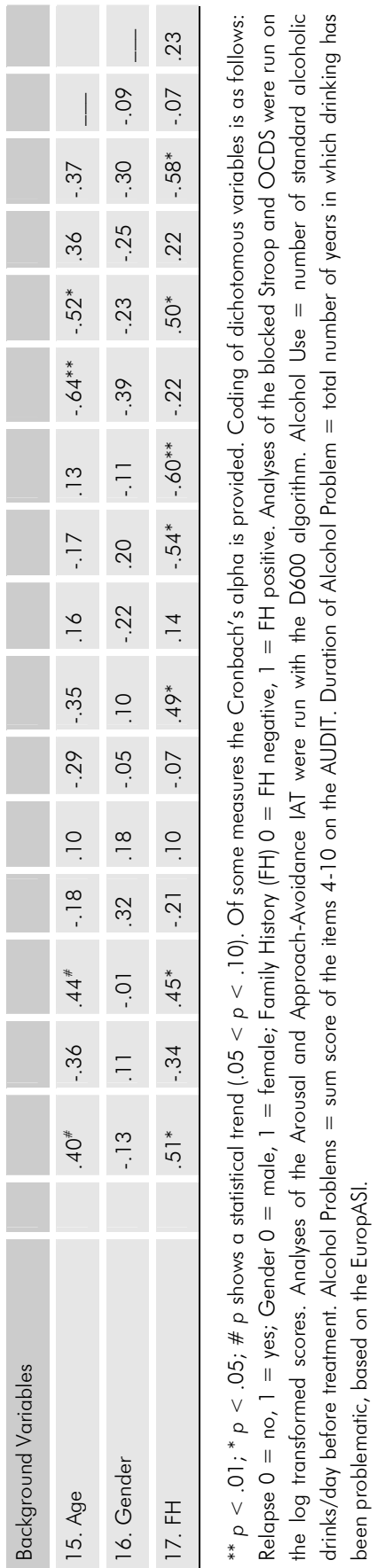




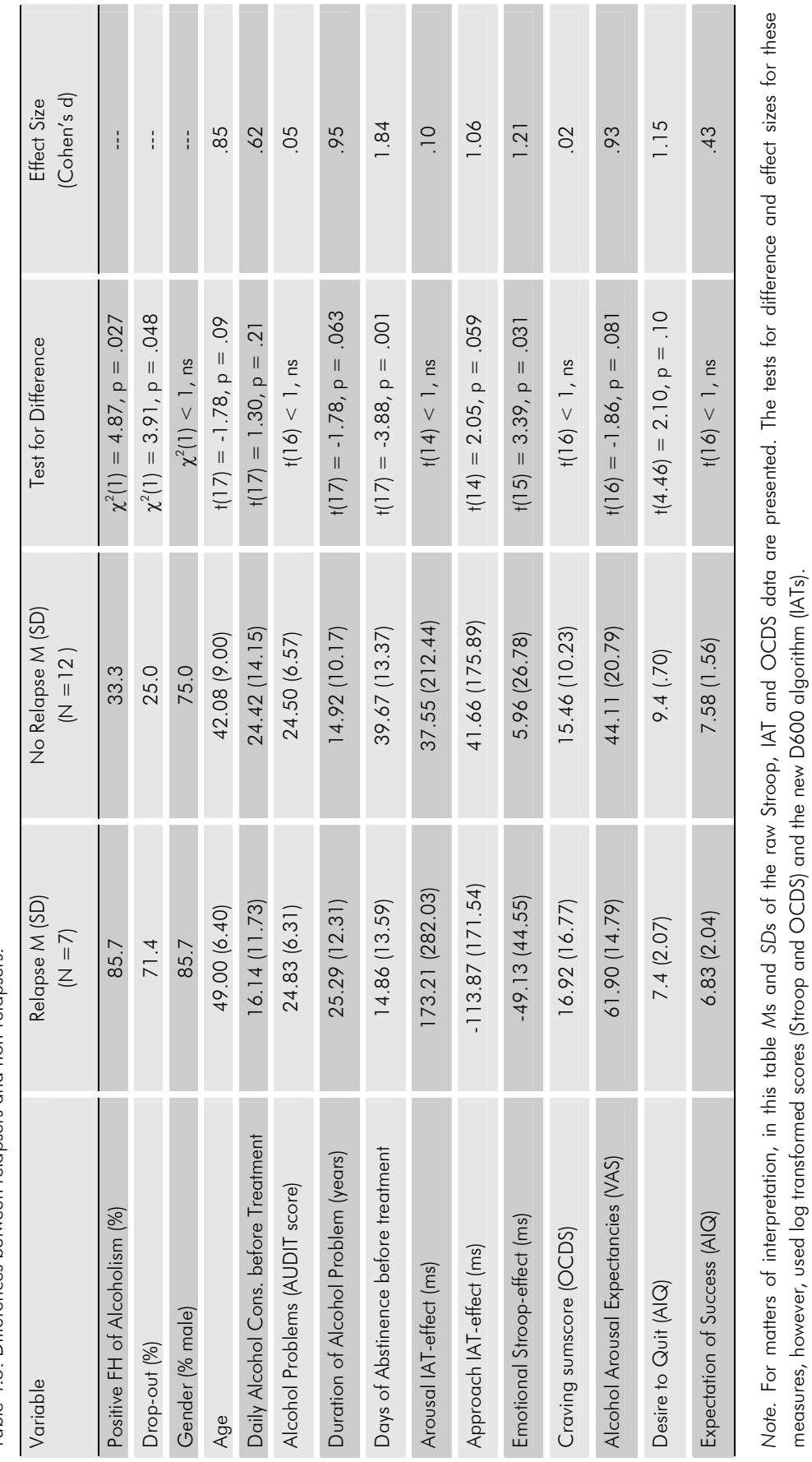


$=.06$ ), stronger explicit arousal expectancies $(p=.08)$ and less desire to quit ( $p=$ .10). They were significantly less days abstinent before treatment than the nonrelapsers $(p=.001)$. Unexpectedly, approach-associations and attentional bias predicted relapse in the reverse way as would be expected: patients who relapsed tended to show stronger alcohol avoidance-associations (a negative IAT-effect, $p=$ .059) and displayed less attentional distraction (a negative emotional Stroop-effect, $p<.05$ ) than patients who were successful at remaining abstinent (Table 4.3). As can be seen in Table 4.2 approach associations and attentional bias were positively correlated $(r=.48, p=.06)$

\section{Time to Relapse}

The backward Cox regression model which was used to predict time to relapse started with the covariates age, $\mathrm{FH}$, duration of the alcohol problem and days of abstinence at step 1 and the explicit arousal expectancies, desire to quit, expectation of success, emotional Stroop and approach-IAT at step 2. The backward procedure resulted in 2 variables predicting time to relapse: duration of the alcohol problem and days of abstinence before treatment. Addition of the remaining variables to exclude masking effects led to a marginally significant effect of the approach-IAT, but overall, this model did not fit better than the first. The final model is shown in Table 4.4. Thus, as duration of the alcohol problem was longer and a patient had been less days abstinent before treatment, the time to relapse became shorter. None of the other variables added significantly in the prediction of time to relapse.

Table 4.4. Cox regression model predicting Time to Relapse

\begin{tabular}{|c|c|c|c|c|c|c|c|}
\hline & \multirow[b]{2}{*}{ B } & \multirow[b]{2}{*}{ SE } & \multirow[b]{2}{*}{ Wald } & \multirow[b]{2}{*}{$p$-value } & \multirow[b]{2}{*}{ Hazard Ratio } & \multicolumn{2}{|c|}{$95 \% \mathrm{Cl}$ for $\operatorname{Exp}(\mathrm{B})$} \\
\hline & & & & & & Lower & Upper \\
\hline Duration Alcohol Problem & .11 & .056 & 3.69 & .055 & 1.11 & 1.00 & 1.24 \\
\hline Days Abstinent & -.13 & .052 & 6.64 & .010 & .88 & .79 & .97 \\
\hline
\end{tabular}

\section{Percentage of Drinking Days}

As can be seen in Table 4.2, percentage of drinking days was related to a shorter time to relapse, less desire to quit and a FHP. A higher percentage of drinking days was furthermore marginally associated with being older, less attentional distraction on the emotional Stroop, stronger explicit arousal expectancies, a lower expectation of success, a longer duration of the alcohol problem and less days of abstinence before treatment. 


\section{Explorative treatment analyses}

After 6 weeks of treatment, 12 patients were still participating in the study, 6 FHNs and 6 FHPs. Of these patients a second measurement of the IATs, Stroop, arousal expectancies, craving and motivation was obtained. Statistical analyses were not possible due to a lack of power, but for exploratory purposes, to generate hypotheses, the data over the course of the first 6 weeks of treatment of the 12 patients is graphically presented (see Figures 4.1A-G). Because all FHNs had remained abstinent, the data were divided into 3 groups: FHNs who remained abstinent $(N=6)$, FHPs who remained abstinent $(N=3)$ and FHPs who relapsed $(N=3)$.

1A) Arousal IAT

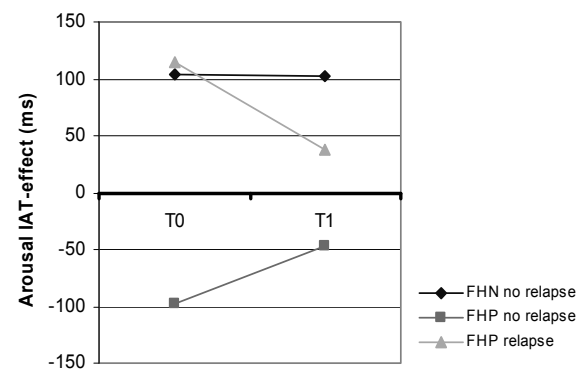

1B) Approach-Avoidance IAT

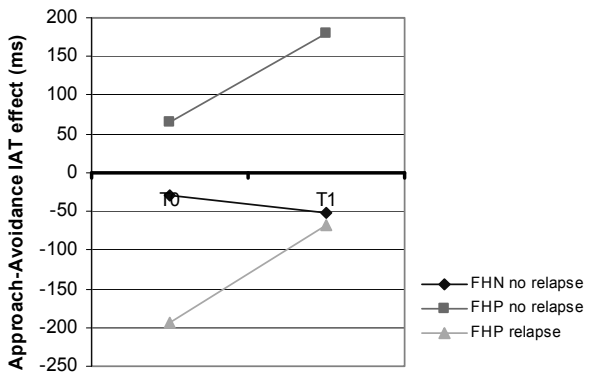

1C) emotional Stroop

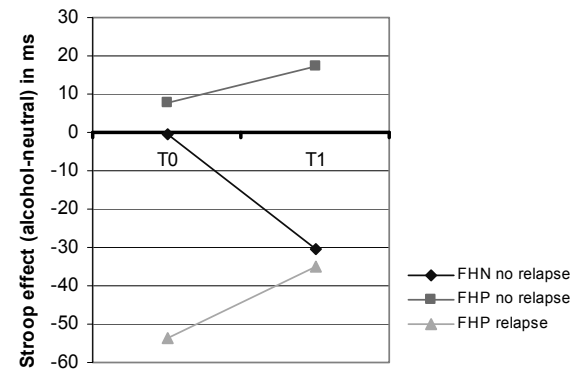

Figure 4.1 (A-C). Outcomes on the three implicit measures are depicted for the $3 \mathrm{FH}$ groups, pre-treatment (TO) and after 6 weeks of treatment (T1). Exploratively, the data seem to suggest that, of all patients, FHPs who relapsed had the strongest implicit avoidance associations (1B) and were the least distracted by alcohol-related words on the emotional Stroop (1C). 
When we look at the graphs, certain patterns can be observed. Figure 4.1E seems to suggest that patients who remained abstinent revealed a decrease in craving over the first 6 weeks of treatment, whereas the patients who relapsed even showed a slight increase. Inspection of the medication compliance data revealed that of the FHPs who had relapsed, 1 had been compliant, 1 had not been compliant and of 1, compliance could not be determined due to a lack of returned pill boxes. The FHPs and FHNs who had been successful at remaining abstinent had all been compliant to naltrexone and all showed a decrease in craving. It is therefore possible that lack of medication compliance influenced relapse.

1D) Arousal Expectancies

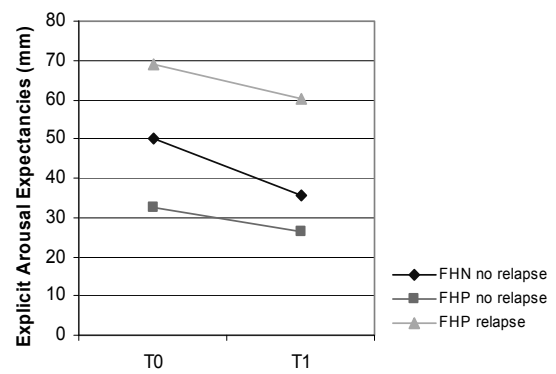

1F) Desire to Quit

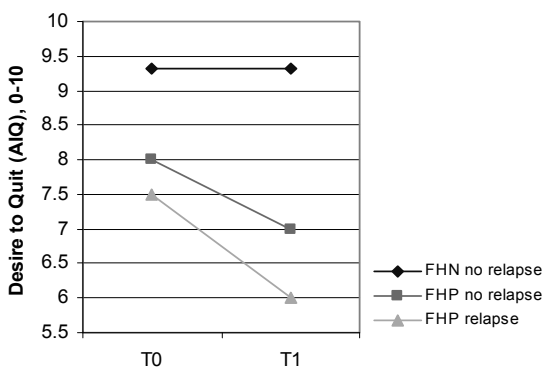

1E) Craving

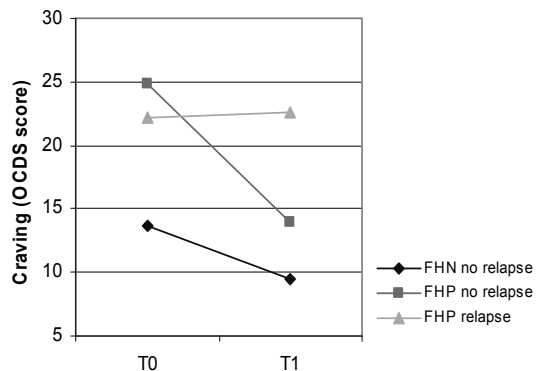

1G) Expectation of Success

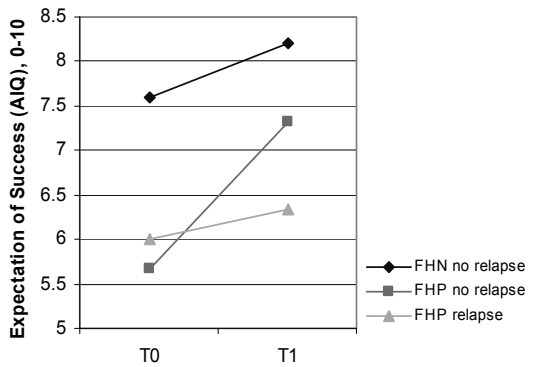

Figure 4.1 (D-G). Outcomes on the four explicit measures are depicted for the $3 \mathrm{FH}$ groups, pre-treatment (TO) and after 6 weeks of treatment (T1). Exploratively, the data seem to suggest that, of all patients, FHPs who relapsed reported the strongest explicit arousal expectancies (1D), no decrease in craving (1E), the least motivation to quit drinking (1F) and the lowest success expectations (1G). 
Figures $4.1 \mathrm{~B}$ and $4.1 \mathrm{C}$ suggest that, of all patients, the FHPs who relapsed had the strongest avoidance associations and were the least distracted by alcoholrelated stimuli. Furthermore, this group seemed to report the strongest explicit arousal expectancies (Figure 1D), the least desire to quit (Figure 4.1F) and the smallest expectation of success in achieving this goal (Figure 4.1G). It even seemed that the $\mathrm{FHNs}$ were, on average, more motivated to quit drinking, with a higher expectation of success in achieving this goal, than FHPs.

\section{DISCUSSION}

This study was aimed at predicting treatment response in alcohol-dependent patients receiving naltrexone treatment. Three dependent variables of main interest were: relapse, time to relapse and percentage of drinking days. Despite the fact that the patients were treated with naltrexone, it was predominantly FHPs who relapsed during treatment (6 out of 7 were FHPs). Monteresso et al. (2001) found that as familial loading of alcohol problems increased, patients benefited more from naltrexone treatment, reflected by less heavy drinking days. This would be expected based on the finding that the more sensitive beta-endorphin response in FHPs is strongly heritable (Froehlich et al., 2000) and naltrexone blocks this response from having its stimulating effect. The present findings appear to be at odds with this notion, but it should be emphasized that the patient sample here was very small. Still, also in the Monterosso study there were FHPs who relapsed while receiving naltrexone. Apparently, some FHPs respond better to naltrexone than others. A large placebo-controlled clinical trial with 627 alcohol-dependent veterans did not find evidence for a better treatment outcome on naltrexone in FHPs compared with FHNs (Krystal et al., 2001). In fact, in that study, no evidence for the effectiveness of naltrexone was found at all compared to placebo. It should be noted that this sample consisted of severely affected patients with long histories of alcoholism which might therefore not be suitable for generalizations to a population consisting of less severe alcoholics for whom naltrexone possibly might be more effective. Also in the present study, patients who relapsed (majority FHPs) tended to be somewhat older (mean age of 49) than the non-relapsers (mean age of 42) and were suffering from long alcoholism histories (approximately 25 years versus 15 years in the no relapse group). When comparing the 3 FHPs who relapsed with the $3 \mathrm{FHPs}$ who did not relapse, the relapsers also tended to be older (51 versus 37 years, $p=.052$ ). It is possible that naltrexone is more effective in younger patients with less severe histories of alcoholism. There is preliminary evidence that naltrexone reduces craving as well as alcohol consumption in adoles- 
cent alcoholics (e.g. Deas et al., 2005). The study sample of Monterosso was 46 years on average $(S D=11.5)$. It would be interesting to further investigate whether the younger patients within the group of FHPs benefited most from naltrexone compared with the older FHP patients. This would imply that it is recommended to start naltrexone treatment in FHPs as early as possible in FHP alcoholics.

Most clinical trials compare relapse rates between patients receiving naltrexone versus placebo. However, it might be interesting to further explore the differences between the successful and less successful patients within the naltrexone group. Several meta-analyses have shown that naltrexone is overall more effective than placebo (e.g. Bouza et al., 2004; Roozen et al., 2005), thus, the next step should be identifying predictors of a good response to naltrexone. Oslin and colleagues (2001) showed that alcohol-dependent patients with a variant or polymorphism of the mu-opioid receptor gene (OPRM1), responded better to naltrexone (which blocks this receptor) than patients carrying the regular allele combination. Heavy drinkers with this polymorphism have been shown to report more craving after exposure to an alcoholic beverage (Van den Wildenberg et al., 2007), which might be dampened by naltrexone. $\mathrm{FH}$ as an indication of genetic risk and a predictor of a good treatment response to naltrexone might be too broad; resulting in finding a lack of effectiveness of naltrexone in all FHPs. Genotyping patients at specific genetic polymorphisms might enhance optimal matching of patient and medication since different types of medication attach to different brain receptors which function or expression can be influenced by different genes.

Besides the finding that the majority of the patients who relapsed were FHPs, another remarkable finding of the present study was that the relapsers tended to show stronger implicit alcohol avoidance associations on the IAT and were less attentionally distracted by alcohol-related words than the patients who remained abstinent. This pattern was still visible within the FHP group after 6 weeks of treatment. Thus, it does not seem that naltrexone affected these automatic processes. One would expect relapse to be associated with alcohol approach associations and an attentional bias for alcohol-related stimuli. How could this reversed pattern be explained? In the anxiety literature, it has often been found that stimuli with a, for the individual, threatening content result in slower reaction times on the emotional Stroop (e.g. Williams et al., 1996). This corresponds to what is usually found in addicted individuals when they perform the addiction Stroop. However, a study on processing bias for spider-related stimuli in children found faster responses to spider words than to neutral words (Morren et al., 2003). It was suggested that the children had found a way to avoid the threatening stimuli (cf. Mogg and Bradley, 1998). Possibly, the alcohol-dependent patients in our sample who relapsed were overall more anxious to relapse and felt threatened by the exposure to alcohol- 
related stimuli. This seems to be reflected in the approach-avoidance IAT: patients who relapsed displayed a strong alcohol-avoidance effect. By responding faster to the alcohol-related emotional Stroop words, avoidance of the threatening stimuli would be successfully obtained because the word disappears from the screen as soon as a response is generated. Note that all participants first performed a mixed version of the Stroop (not reported here) in which the same neutral and alcoholrelated words were used. The previous practice of responding to alcohol-related words might have speeded up their response to these words. It remains speculative, but possibly, these avoidant patients also try to avoid exposure to alcohol-related stimuli in real life, which might prevent them from having the opportunity to practice with high-risk situations, rendering them more vulnerable to relapse in case they encounter one.

It should be mentioned that, contrary to our hypothesis, the arousal-IAT did not predict either relapse, time to relapse or percentage of drinking days. Also, the explorative analyses over time did not reveal any indication of a treatment effect. Despite disadvantages of a bipolar IAT such as relative measurement (e.g. associations between alcohol and arousal can be due to associations between soda and sedation), a bipolar IAT might be preferred over unipolar measurement. It is for example still not entirely clear whether the attribute category 'neutral' is the most optimal contrast category in the unipolar IAT. Furthermore, often higher reliabilities are found for bipolar IATs compared with unipolar IATs (Houben, 2007), as is shown in this study as well.

One of the strongest predictors of relapse and time to relapse was the number of days a patient had been abstinent before treatment, a finding which is not unexpected since previous behavior is often one of the strongest predictors of future behavior. Days of abstinence was strongly related to $\mathrm{FH}$, with the $\mathrm{FHNs}$ being twice as long abstinent than the FHPs.

A concept such as craving, which would usually be expected to be strongly related to relapse (e.g. Addolorato et al., 2005) did, at treatment admission, not contribute significantly to differentiating between relapsers and non-relapsers or to the prediction of time to relapse or percentage of drinking days, whereas the more automatic cognitive processes such as avoidance associations and attentional distraction showed a stronger predictive value. An association between craving at treatment admission and relapse was fully absent (see Table 4.2). The more explorative analyses over time suggested that the FHPs and FHNs who had remained abstinent, reported a reduction in craving six weeks later, whereas the FHPs who relapsed showed a slight increase. The lack of a reduction in craving and a possible subsequent relapse might be related to the lack of compliance to the medication. Medication compliance has proven to be important in the effectiveness of 
naltrexone (Pettinati et al., 2000). Non-compliant patients show relapse rates that are comparable to those of patients receiving placebo (approximately $40 \%$ ).

Besides the significant effects of a FHP, less days of abstinence, less attentional bias and the borderline significant effect of avoidance associations, relapse tended to be associated with the presence of stronger explicit arousal associations. Based on a finding in a large sample of alcoholics, that FHPs showed stronger positive expectancies for a high dose of alcohol than FHNs, Wiers and colleagues (2000) predicted better response to naltrexone for these individuals. This prediction was not confirmed in the present (small) sample. Individuals with the strongest explicit arousal expectancies relapsed more often. Treatment did not seem to have changed these explicit cognitions, although a slight decrease is visible in all patients (4.1D).

Finally, the Alcohol Intentions Questionnaire revealed that relapse tended to be associated with a lower desire to quit drinking at treatment admission. Stronger effects were found when comparing FHPs and FHNs with respect to desire to quit drinking and expectation of success in achieving this goal. FHPs tended to have a lower desire to quit drinking in the first place and furthermore, they expected to be less successful at doing this. This distinction between FHPs and FHNs remained visible over the course of treatment. Thus, the finding that the majority of patients who relapsed were FHPs seems to be related to motivational factors. Figures $1 \mathrm{~F}$ and $1 G$ reveal a pattern where FHPs who relapsed reported the least desire to quit drinking and the lowest success expectation compared with the other two groups who did not relapse. Although the sample was very small, this could be an interesting finding because FHPs who seek treatment might in general be less motivated to change their drinking patterns at the start of treatment. If motivation and selfefficacy are effectively targeted in this particular group, the chance of relapse might be decreased.

Several limitations to the study should be mentioned. First, the eventual sample size was very small and second, there was a lack of a control group (all patients were receiving naltrexone). Both were due to problems with regard to patient recruitment. The trial started out as a randomized, double-blind, placebo-controlled clinical trial, but we experienced severe problems with patient recruitment (one problem was that the rumor spread that patients who participated ran the risk of receiving placebo, while naltrexone is a medication already approved of, and thus, could be received if one did not participate in the trial). In reaction to these problems, it was decided to drop the placebo condition. As such, the trial became an open label trial in which all participants knew they were receiving naltrexone. This limits the generalizations that can be done with regard to the outcomes of the study. 
However, little or no research has so far been conducted on predicting treatment response with implicit measures. Despite a relatively small study sample of only 23 patients, Cox et al. (2002) still found interesting results in predicting relapse and dropout. Also, the present sample of 19 patients revealed differences between patients who relapsed and those who did not. In addition, the large smoking study of Waters et al. (2003) showed promising results in predicting relapse with an attentional bias measure, as did the study from Marissen et al. (2006) in heroin-dependent patients. Implicit measures appear to tap relatively automatic processes which can influence behavior, but which may not be readily assessed with a questionnaire. It is recommended to assess these automatic associations or processes in the clinic to enhance the prediction of treatment outcome, thereby offering a possibility to decrease the chance of relapse in those individuals who are at increased risk. Further research is needed to investigate how these automatic associations influence treatment success and how they might be changed. It remains speculative, but it would be interesting to investigate whether patients with alcohol-avoidance associations and a lack of attentional distraction by alcoholrelated words would benefit from cue exposure treatment to offer them the opportunity to practice with high-risk situations they might be anxious of, which might place them at an increased risk for relapse. Additionally, increasing motivation to quit drinking and improving feelings of self-efficacy that this goal can be achieved might be particularly beneficial in FHPs. The AIQ could be a valuable tool to administer at the start of treatment, although one might run the risk of receiving socially desirable answers. The same advantage and disadvantage holds for the assessment of explicit positive alcohol arousal expectancies. Aiming at reducing these during treatment could be potentially beneficial. Further research is warranted to find good predictors of the treatment response to naltrexone. Research in relation to the mu-opioid receptor polymorphism seems promising.

\section{ACKNOWLEDGEMENTS}

The authors would like to acknowledge all the physicians and addiction clinic employees for the recruitment of the patients at the different clinics. Furthermore, we would like to thank the research assistants Femke van Lambaart and Tim Satijn for collecting part of the data. This research was funded by ZONMW (grant 31000065); RWW is funded by a 'VIDI' grant from the Dutch National Science Foundation (N.W.O., grant 45202005). 
Appendix: IAT stimuli

\section{Target stimuli}

Alcohol: wine, beer, whisky, port, vodka and rum.

Soda: Cassis, Sinas (both lemonades), Spa (sparkling water), Coke, tonic, and juice.

Arousal Attribute stimuli:

Active: talkative, jovial, restless, alert, unrestrained, rambunctious

Neutral: constant, wide, brown, digital, recent, historic

Approach-avoidance Attribute stimuli:

Approach: Toward, to touch, to grasp, to grab, to approach, ahead

Avoidance: From, to run away, to escape, to leave, to flee, departure 



\section{CHAPTER 5}

\section{A FUNCTIONAL POLYMORPHISM OF THE MU-OPIOID RECEPTOR GENE (OPRMT) INFLUENCES CUE-INDUCED CRAVING FOR ALCOHOL IN MALE HEAVY DRINKERS}

Published as: Van den Wildenberg, E., Wiers, R. W., Dessers, J., Janssen R. G. J. H., Lambrichs, E. H., Smeets, H. J. M., \& Van Breukelen, G. J. P. (2007). A Functional Polymorphism of the Mu-Opioid Receptor Gene (OPRM1) Influences CueInduced Craving for Alcohol in Male Heavy Drinkers. Alcoholism: Clinical and Experimental Research, 31, 1-10. 


\section{ABSTRACT}

Background: The mu-opioid receptor gene (OPRM1) codes for the mu-opioid receptor which binds beta-endorphin. The A1 18G polymorphism in this gene affects beta-endorphin binding such that the Asp40 variant ( $G$ allele) binds betaendorphin three times more tightly than the more common Asn40 variant (A allele). This study investigated the influence of the A1 18G polymorphism on cue reactivity after exposure to an alcoholic beverage in male, heavy drinkers.

Methods: Participants were either homozygous for the A allele $(n=84)$ or carrying at least one copy of the $G$ allele $(n=24)$. All participants took part in a cue reactivity paradigm where they were exposed to water and beer in 3-minute trials. Dependent variables of main interest were subjective craving for alcohol, subjective arousal and saliva production.

Results: $G$ allele carriers reported significantly more craving for alcohol than the $A$ allele participants (as indicated by the within-subject difference in craving after beer versus after water exposure). No differences were found for subjective arousal and saliva. Both groups did not differ in family history of alcoholism. Participants with the $G$ allele reported a significantly higher lifetime prevalence of drug use than participants homozygous for the A allele.

Conclusions: A stronger urge to drink alcohol after exposure to an alcoholic beverage might contribute to a heightened risk for developing alcohol-related problems in individuals with a copy of the $G$ allele. The $G$ allele might also predispose to drug use in general.

Key words: Alcohol; Cue Reactivity; Craving; OPRM1; Polymorphism 


\section{INTRODUCTION}

Alcoholism continues to be one of the most prevalent psychiatric disorders world wide (NIAAA, 2005). Research during the past decades has shown that a large genetic component is involved in the development of alcohol use disorders (McGue, 1999). Children of alcoholics have a two to nine fold increased risk of alcohol-related problems, partly because of their genetic predisposition (McGue, 1999). One system that is very important in (alcohol) addiction is the endogenous opioid system (Herz, 1997).

Alcohol consumption increases the levels of endogenous opiates such as betaendorphin in the mesolimbic reward system of the brain. This in turn, leads to the release of dopamine in the nucleus accumbens, one of the main nuclei involved in the rewarding and reinforcing effects of addictive substances (Herz, 1997; Robinson and Berridge, 2003). Lower basal activity of beta-endorphin has been found in individuals with a positive family history (FHP) compared with individuals without this genetic liability (FHN) which suggests a state of 'underarousal' in FHPs (Dai et al., 2002; Gianoulakis et al., 1996a). After drinking a relatively high dose of alcohol in a short period of time, the plasma level of beta-endorphin rises in FHPs, but not in FHNs (Gianoulakis et al., 1996a; 1996b), which may indicate enhanced sensitivity to effects of alcohol in FHPs. Froehlich et al. (2000) demonstrated in a twin study that, compared with other hormonal responses to alcohol consumption, the response of beta-endorphin was the only response found to be strongly heritable. Thus, one might conclude that the beta-endorphin response could function as a biomarker or endophenotype (Gottesman and Gould, 2003) of the sensitivity to the rewarding effects of alcohol. Several randomized clinical trials with alcoholdependent patients have shown that the opioid antagonist naltrexone reduces craving for alcohol, the number of drinking days and drinks per drinking day and increases the time to relapse and the number of days of abstinence (for a review see Bouza et al., 2004). Monterosso et al. (2001) found that a FHP predicted a better response to naltrexone (more abstinence and less craving) compared with a FHN, emphasizing the importance of a genetic contribution in the beta-endorphin response.

Bond et al. (1998) investigated the role of a genetic contribution to the sensitivity of the beta-endorphin system by looking at the single nucleotide polymorphism (SNP) at position 118 (A1 18G) in the coding region of the mu-opioid receptor gene (OPRM1). This SNP codes for a change in amino acids from asparagine (Asn) into aspartic acid (Asp) at position 40, which results in a 3 times stronger binding of beta-endorphin to the mu-opioid receptor. Because of alcohol's stimu- 
latory effect on beta-endorphin, it might be expected that individuals carrying at least one copy of the $G$ allele would also display a stronger sensitivity to the effects of alcohol. However, findings of association studies regarding the importance of the OPRM 1 gene in alcohol and substance dependence have been mixed (e.g. Bart et al., 2005; Bergen et al., 1997; Hoehe et al., 2000). A recent metaanalysis, involving more than 8000 participants, concluded that the A118G polymorphism does not contribute to a heightened risk for substance dependence (Arias et al., 2006). Other research suggests that the $G$ allele is associated with reduced mu-opioid receptor expression, indicating a functional loss (Zhang et al., 2005).

Ray and Hutchison (2004) conducted a study in which they compared the response to intravenous alcohol administration between healthy individuals carrying two A alleles and individuals carrying at least one copy of the $G$ allele. They found that individuals with the $G$ allele were more sensitive to the effects of alcohol compared with individuals homozygous for the A allele. Participants with the less common $G$ allele experienced stronger subjective feelings of stimulation, happiness, sedation and intoxication after the alcohol administration. Furthermore, three times as many FHPs were found in the $G$ allele group than in the A allele group (but their sample was relatively small, $23 \mathrm{~A}$ versus $15 \mathrm{G}$ allele participants). After controlling for $\mathrm{FH}$, the results mentioned above remained unchanged. Retrospective DNA analysis of a subset of participants from 3 separate clinical trials with alcoholdependent patients showed that patients treated with naltrexone carrying a copy of the $G$ allele had significantly lower relapse rates and a longer time to return to heavy drinking compared with patients homozygous for the A allele (Oslin et al., 2003). Taken together, these findings suggest that the A1 $18 \mathrm{G}$ polymorphism might play an important role in the sensitivity of the response to alcohol and might moderate the response to opioid antagonists such as naltrexone.

Not only does the actual intake of alcohol or drugs lead to a release of betaendorphin and dopamine in the nucleus accumbens, but so does exposure to alcohol or drug related cues due to learned responses to these stimuli (Robinson and Berridge, 2003). In humans, this can be tested in cue reactivity paradigms, where the participant is exposed to his or her favorite alcoholic beverage. Rohsenow et al. (1994) demonstrated that in alcohol-dependent patients receiving cue-exposure treatment, strong saliva reactivity during exposure to the alcoholic beverage predicted a higher number of drinking days at follow-up. Other studies found that naltrexone reduced the urge to drink after cue exposure, both in heavy drinkers (Palfai et al., 1999) and in alcohol-dependent men (Rohsenow et al., 2000). A randomized cue exposure study (placebo vs naltrexone) in non-treatment seeking heavy and dependent drinkers by McGeary et al. (2005) investigated the difference 
in reactivity between individuals with and without a $118 \mathrm{G}$ allele. They did not find the suspected differences between both groups with regard to urge ratings for alcohol. Contrary to their hypothesis, participants with a $G$ allele reported higher cue-elicited urge ratings on naltrexone compared with placebo. In participants homozygous for the common A allele, no differences in craving between the placebo or naltrexone condition were found. Taken together, preclinical studies suggest an important role of the $G$ allele in the sensitivity to alcohol. With regard to association and cue reactivity studies the findings have been mixed. Thus far, not much research with the A118G SNP has been done in healthy, heavy drinking individuals. Therefore, the aim of this study was to investigate the relationship between the A1 18G polymorphism and cue reactivity in heavy drinking individuals.

This study was designed to investigate the hypothesis that individuals with a copy of the $G$ allele display stronger cue-reactivity when exposed to an alcoholic beverage compared with participants homozygous for the A allele. That is, the effect of genotype was expected to be larger in the alcohol trial compared with the water trial and the beverage effect (alcohol-water) was expected to be larger for the $G$ allele participants compared with the A allele participants. This was hypothesized for subjective craving, subjective mood (experienced arousal) and salivary reactivity. Furthermore, based on the findings of Ray and Hutchison (2004), it was expected that individuals with a copy of the $G$ allele would more often report a positive $\mathrm{FH}$ of alcoholism than participants homozygous for the A allele. Finally, given mixed evidence, we investigated whether participants with a $G$ allele would report more drug use in general.

\section{MATERIALS AND METHODS}

\section{Participants}

Participants were 109 male heavy drinkers who were recruited via flyers at several faculties of the University of Maastricht and a higher professional education school. After they indicated by email they were interested in participating, they were screened in a telephonic interview. Inclusion criteria were as follows: age range between 18-28 years; a minimum of 20 standard alcoholic consumptions per week; at least one binge episode (6 or more drinks) in the past two weeks and the participant as well as his parents were required to be from Dutch origin to prevent population admixture. The exclusion criteria were as follows: dyslexia and color blindness (related to another study in which the participants took part). After the experiment each participant received a monetary compensation of $€ 15$. 


\section{Procedure}

The study was approved of by the Medical Ethics Committee of the Academic Hospital in Maastricht. Written informed consent was given before the start of the experiment.

Participants were seated in front of individual tables, separated from each other by screens, in such a manner that they could not see each other. A maximum of 5 participants could be tested this way at the same time. The participants were instructed that it was very important to perform all the actions pointed out by the experimentor in unison. This was practiced during the baseline measurements. In front of the participants were two inverted opaque, tinfoil containers under which the beverages were hidden. The container on the left side always contained an empty glass and a bottle of commercially labeled spring water ("Spa Blauw"). The container on the right side always contained an empty beer glass and a bottle of "Brand" beer (all participants had previously indicated beer was their favorite alcoholic drink). Before the cue-exposure trials started, baseline measures of subjective mood, subjective craving and saliva were taken. Based on the cue exposure paradigm by Monti et al. (1987) and Rohsenow et al. (2000) all participants first received a 3-min relaxation period. After this the cue reactivity trials started. These were always in a fixed order (water, alcohol) to prevent transfer effects (Monti et al., 1987). Participants were asked to place the three dental rolls in their mouth and then they were asked to lift the left container, take the empty glass and fill it up half with water from the bottle. After this the 3-min exposure trial started. Participants were asked to bring the glass up, take a good look at it and sniff it for 5 seconds, with a total of 15 times in $3 \mathrm{~min}$. After 3 min they were asked to put the glass down and cover it with the container. Then, they removed the cotton rolls, put them back in the little plastic bag and sealed it well to prevent saliva loss. They were handed a plastic cup to take a sip of water to prevent a dry mouth due to the cotton rolls. Next, the participants completed the Affect Grid (to measure mood), the Visual Analogue Scales (VAS) of craving for water and alcohol and the Desire for Alcohol Questionnaire (DAQ). After all participants had completed the questionnaires the same procedure was repeated for the alcoholic beverage under the container on the right side.

In order to determine genotype, buccal cells were collected by omni swab. Participants returned a second day for another part of the experiment where they filled out the Drug use questionnaire. 


\section{Materials and Measures}

\section{Pretest Measures}

Alcohol Use. Alcohol use was measured with a self-report questionnaire (Wiers et al., 1997) based on the reliable and validated Time-Line Follow-Back method (Sobell \& Sobell, 1990). Participants filled out their alcohol consumption of each day of the week prior to the experiment and indicated whether this was more, less or equal to what they would typically drink on that day of the week. Furthermore, the number of binges ( 6 drinks or more on one occasion) of the past two weeks was reported. A standard drink contains $10 \mathrm{~g}$ alcohol/unit.

Alcohol-related Problems. Two questionnaires were used to measure alcoholrelated problems. The 18-item Rutgers Alcohol Problem Index (RAPI; White \& Labouvie, 1989) measures how often several social and health-related problems occur. The 18-item version we used correlates 99 with the 23-item version (White and Labouvie, 2000). Scores were multiplied by 23 and divided by 18 in order to make them comparable to the 23-item version. Furthermore, the 10-item Alcohol Use Disorder Identification Test (AUDIT; Saunders et al., 1993) was used which measures alcohol consumption (item 1-3) and alcohol-related problems (item 410).

Drug Use. The use of ten other drugs was also assessed: Cigarettes, Cannabis, Amphetamines/Speed, XTC/MDMA, Cocaine, Hallucinogenic drugs (e.g. magic mushrooms, smartdrugs, LSD), Heroin, Medicines/Pills (e.g. sedatives, benzodiazepines), other Opiates/Analgesics (e.g. methadone) and Inhalants (e.g. laughing gas, poppers, glue). It was asked if they had ever used the drug and, if yes, at which age they first used it. Based upon the question if the participant had ever used any of the 10 different drugs, a sum score was calculated ranging from 0 (never used any of the drugs) to 10 (ever used all of the 10 drugs). Next, it was asked how often they had used the drug during the past week, month and year. Three additional sum scores of the 10 types of drugs were calculated: the higher the score, the more often they had used the drug during the past week, month and year.

Family History of Alcoholism. Participants filled out how many first-degree and second-degree relatives they had and answered the following question for each of these relatives: "Does/did this relative have a serious alcohol problem for which he or she should be/have been treated?". If the answer was 'yes', follow-up questions regarding this person's alcohol problem were asked (based on the Familiy History 
Tree by Mann, Sobell, Sobell, \& Pavan, 1985). A participant was categorized as FHP when at least one first-degree family member suffered from alcohol dependence.

\section{Cue Reactivity Measures}

Craving. Craving for water and craving for alcohol were assessed by means of two $100 \mathrm{~mm}$ VAS with the anchors "absolutely no urge" and "an irresistible urge". Furthermore, a Dutch translation (Franken et al., 2003) of the DAQ (Love et al., 1998) was used in order to obtain a more specific assessment of cue-elicited craving for alcohol. The DAQ is a 14-item questionnaire that measures the desire to drink alcohol at the moment of testing itself. The scale consists of four subscales: (1) Strong Desires and intentions to drink; (2) Negative Reinforcement; (3) Control over drinking and (4) Mild Desires to drink. As response format, 14 VAS ranging from $0 \mathrm{~mm}$ (strongly disagree) to $70 \mathrm{~mm}$ (strongly agree) were used instead of 7 point Likert scales, to prevent memory for the marked position after repeated administrations. As outcome, the mean score across all 14 items was used. Cronbach's a was found to be .84 (on the baseline DAQ).

Mood. The affect grid (Russell, Weiss, \& Mendelsohn, 1989) was used to assess mood. The grid is a $9 \times 9$ square consisting of a valence dimension ranging from 'unpleasant feelings' (left side of the grid) to 'pleasant feelings' (right side) and an arousal dimension ranging from 'high arousal' (top) to 'sleepiness' (bottom). The participant placed an $\mathrm{X}$ in one of the 81 possible positions. Corners and sides from the grid contained affect descriptors to elucidate which mood state belongs to which part of the grid. Based on the position of the $X$, one valence and one arousal score were computed, both ranging from 1 to 9 . The affect grid was found to have adequate reliability and validity (Russell et al., 1989).

Salivation. Measurement of saliva reactivity was based on a standard measure, the Strongin, Hinsie and Peck technique (in White, 1977), in which three cotton rolls are placed in the mouth (one sublingually and two buccally). Before and after the 3-min cue exposure trial, weight of the cotton rolls was determined. The weight difference indicated the amount of saliva that was produced during cue exposure. The cotton rolls were put in small-sized plastic bags that were sealed to prevent loss of saliva. 


\section{Genotyping}

Genomic DNA was isolated from from buccal cells (2 Omniswabs per sample, Whatman, The Netherlands) with the QIAamp DNA Mini Kit (QIAgen, The Netherlands). For both procedures the manufacturers protocols were followed. DNA concentration and purity were measured with a Nanodrop spectrophotometer.

Determination of the OPRM1 A1 18G (rs 1799971) genotype was performed by sequencing. First, a Polymerase Chain Reaction (PCR) generating a 293 bp fragment was carried out with primers: 5'- GAAAAGTCTCGGTGCTCCTG -3' (upper) and 5' -GGAGTAGAGGGCCATGATCG -3' (lower). PCR was performed in 96-well microtiterplates on a Biometra T1 Thermocycler (Westburg, Leusden, The Netherlands). We used approximately $10-50 \mathrm{ng}$ of genomic DNA in a $25 \mu \mathrm{l}$ reaction mixture containing 1x PCR buffer (Invitrogen, Breda, The Netherlands), $0.2 \mathrm{mM}$ dNTPs, $0.1 \mu \mathrm{M}$ of each primer, $1.5 \mathrm{mM} \mathrm{MgCl}_{2}$, and $1 \mathrm{U}$ Taq DNA polymerase (Invitrogen, Breda, The Netherlands). Cycling conditions were: initial 5 min. denaturation at $95^{\circ} \mathrm{C}$; 35 cycles of denaturation at $94^{\circ} \mathrm{C}$ for $30 \mathrm{sec}$., annealing at $60^{\circ} \mathrm{C}$ for $1 \mathrm{~min}$. and extension at $72^{\circ} \mathrm{C}$ for $1 \mathrm{~min}$. followed by a final extension for $7 \mathrm{~min}$. at $72^{\circ} \mathrm{C}$. PCR products were purified using Multiscreen-PCR Plates according to the manufacturers protocol (Millipore, The Netherlands). Subsequently, $1 \mu \mathrm{l}$ of purified PCR product was cycle-sequenced using $2 \mu \mathrm{l}$ BigDye Terminator v3.1 Ready Reaction Mix, $3 \mu$ l BigDye Terminator v3.1 Sequencing Buffer (both Applied Biosystems, The Netherlands) and $1 \mu \mathrm{l}$ of PCR primer (upper or lower, $3.2 \mathrm{pmol}$ ) in a total volume of $20 \mu \mathrm{l}$ in a Biometra T1 Thermocycler. Cycling conditions are according to the manufacturers protocol. Extension products were purified by ethanol precipitation following the manufacturers procedure and size-resolved on an ABI3100 Genetic Analyzer, using $36 \mathrm{~cm}$ capillaries filled with POP6 polymer. The sequence traces were analysed using Sequencing Analysis software version 3.7 (Applied Biosystems, The Netherlands). Two researchers independently scored genotypes, and a third expert judged discordant results before entering them into the database.

\section{Outcome Analyses}

The study design was a within-subject (Beverage: Water vs Alcohol) by betweensubject (Genotype: AA vs AG/GG) factorial design with between-subject covariates age, alcohol use and baseline of the outcome at hand, and was analyzed accordingly, using repeated measures ANOVA (SPSS version 12.0.1). For each of the six outcome variables ( 3 for subjective craving, 2 for mood and 1 for saliva) the initial model included interactions of genotype with each covariate. Each covariate was first centered by subtracting the total sample mean from it, to prevent possible col- 
linearity and to allow interpretation of the within-subject effect as beverage effect (alcohol-water) for the average person in the sample, and interpretation of genotype by beverage interaction as interaction for persons scoring average on covariates.

As our main interest was in effects of beverage and genotype, we concentrated on the within-subject ANOVA part, which comes down to an ANCOVA with the beverage effect (outcome difference between alcohol and water) as dependent variable, and genotype and covariates as independent variables. Genotype by covariate interactions were deleted from this model if not significant, using $\alpha=.01$ to correct for multiple testing. If the final model for the within-subject part did not contain any beverage by genotype by covariate interaction anymore and the beverage by genotype interaction was not significant, (using $\alpha=.05$ ), then the between-subject ANOVA part was interpreted. The latter comes down to an ANCOVA with the outcome average of both conditions (exposure trials) as dependent variable. If a beverage by genotype (by covariate) interaction was found, then a separate ANCOVA was run per condition (water, alcohol). In both cases; that is, ANOVA of the average of both conditions, and ANOVA per condition, nonsignificant genotype by covariate interaction was removed from the model before testing main effects'.

\section{RESULTS}

\section{Genotyping}

Genotyping resulted in 84 participants homozygous for the major A allele, 23 participants with the $A G$ combination and 1 participant homozygous for the $G$ allele. One participant was genotyped as AAG. Because the presence of three alleles indicates a possible duplication of part of chromosome 6 or sample contamination this subject was excluded from the analyses. So, allele frequency was .88 for the A allele and . 12 for the $G$ allele. In the analyses the data of the $A G$ and $G G$ participants were combined.

\footnotetext{
${ }^{1}$ Since the genotype groups differed with regard to drug use and the participants were tested in small groups up to 5 people, all analyses were repeated with the centered covariates Drug use, Block (to test the lineair effect of testing in groups) and the square of the variable Block (quadratic effect). None of these analyses revealed any significant drug or block effects. Therefore, these covariates were left out of the final analyses.
} 


\section{Alcohol Use and Problems}

As can be seen in Table 5.1 no differences were found between participants with a copy of the $G$ allele and participants homozygous for the A allele regarding alcohol use and problems (RAPI and AUDIT scores). On average, both groups consumed almost 40 alcoholic drinks per week ${ }^{2}$. RAPI scores fell in the range of a clinical sample for adolescents (White \& Labouvie, 1989). The internal consistency (Cronbach's $\alpha$ ) of the RAPI was .76. For the AUDIT, a cut-off of 11 is used in college samples as an indication of problem drinking (Fleming et al., 1991). With an average AUDIT score of 14.30 (SD = 3.85) this group can clearly be described as problem drinkers. Internal consistency of the AUDIT was .71. Both $\alpha^{\prime}$ s suffer from restriction of range due to the selection of heavy drinkers only.

Table 5. 1. Pre-test differences between the A and G allele group.

\begin{tabular}{|c|c|c|c|}
\hline Variable & $\begin{array}{c}\text { AA Allele (SD) } \\
(N=84)\end{array}$ & $\begin{array}{c}\text { AG/GG Allele (SD) } \\
(N=24)\end{array}$ & Test for Difference \\
\hline Age & $21.44(2.10)$ & $20.58(2.21)$ & $t(106)=1.74, p=.084$ \\
\hline Weekly Alcohol Consumption & $39.27(11.61)$ & $38.58(11.86)$ & $t(106)<1 \mathrm{~ns}$ \\
\hline Number of binges in past two weeks & $5.49(2.21)$ & $5.48(1.68)$ & $t(106)<1 \mathrm{~ns}$ \\
\hline Alcohol Problems (RAPI score) & $24.45(9.53)$ & $23.61(9.38)$ & $t(105)<1 \mathrm{~ns}$ \\
\hline Alcohol Problems (AUDIT score) & $14.30(4.02)$ & $14.30(3.24)$ & $t(105)<1$ ns \\
\hline Family History of Alcoholism (\%) & 7.1 & 8.7 & $\chi^{2}(1)<1 \mathrm{~ns}$ \\
\hline Baseline Craving (DAQ) & $19.54(9.13)$ & $18.49(7.81)$ & $t(106)<1 \mathrm{~ns}$ \\
\hline Baseline Craving Alcohol (VAS) & $30.48(25.66)$ & $35.25(25.41)$ & $t(106)<1 \mathrm{~ns}$ \\
\hline Baseline Craving Water (VAS) & $55.00(23.56)$ & $56.33(24.29)$ & $t(106)<1 \mathrm{~ns}$ \\
\hline Baseline Arousal & $4.44(1.29)$ & $4.75(1.32)$ & $t(106)=-1.03, p=.31$ \\
\hline Baseline Valence & $6.26(1.40)$ & $6.67(1.17)$ & $t(106)=-1.29, p=.20$ \\
\hline Baseline Saliva & $1.96(1.01)$ & $1.85(1.09)$ & $t(106)<1 \mathrm{~ns}$ \\
\hline
\end{tabular}

Note. For the RAPI and AUDIT scores and FH, data of 1 participant was missing.

\section{Drug Use}

The Drug Use scores of both genotype groups are presented in Table 5.2. An independent t-test on the 'Drug use ever' sum score revealed that participants with a

\footnotetext{
${ }^{2}$ Two outliers were found with a weekly alcohol consumption around 90 standard drinks. These values were recoded into the subsequent highest score plus one, resulting in 68.50 drinks. Analyses with and without these 2 outliers did not differ.
} 
copy of the $G$ allele had significantly more often ever used any of the drugs ( $p=$ .036). Separate Chi-square tests for each of the nine drugs (none of the participants had ever used heroin) revealed that the $G$ allele group significantly more often reported having ever used cannabis $(p=.006)$ and amphetamines $(p<$ $.001)$. After Bonferroni correction for multiple testing ( $\alpha=.05 / 9=.0056$ ), the results for amphetamines were still significant, and for cannabis borderline significant. The use of XTC and cocaine ever were in the expected direction but not significant ( $p=.14$ resp., $p=.13$ ). No significant differences were found for the other drugs ( $p s>$.18). Trends were found for $G$ allele participants reporting more overall drug use during the past year $(p=.052)$, past month $(p=.064)$ and past week $(p=.11)$.

Table 5.2. Drug Use differences between the A and $G$ allele group.

\begin{tabular}{|c|c|c|c|}
\hline & $\begin{array}{l}\text { AA Allele (SD) } \\
\qquad(N=84)\end{array}$ & $\begin{array}{c}\text { AG/GG Allele (SD) } \\
(N=24)\end{array}$ & Test for Difference \\
\hline Drug Use ever & $2.24(1.50)$ & $3.05(1.84)$ & $t(103)=-2.13, p=.036$ \\
\hline Cigarettes & 63.9 & 59.1 & $\chi^{2}(1)<1 \mathrm{~ns}$ \\
\hline Cannabis & 75 & 100 & $\chi^{2}(1)=7.45, p=.006$ \\
\hline Amphetamines/Speed & 3.6 & 29.2 & $\chi^{2}(1)=14.56, p<.001$ \\
\hline XTC/MDMA & 19 & 33.3 & $\chi^{2}(1)=2.20, p=.14$ \\
\hline Cocaine & 9.5 & 20.8 & $\chi^{2}(1)=2.26, p=.13$ \\
\hline Hallucinogetic Drugs & 22.6 & 33.3 & $\chi^{2}(1)=1.14, p=.29$ \\
\hline Heroin & 0 & 0 & --- \\
\hline Pills (e.g. Sedatives) & 7.1 & 0 & $\chi^{2}(1)=1.82, p=.18$ \\
\hline Opiates & 2.4 & 0 & $\chi^{2}(1)<1 \mathrm{~ns}$ \\
\hline Inhalants & 21.4 & 33.3 & $\chi^{2}(1)=1.45, p=.23$ \\
\hline Drug Use past year & $2.96(2.67)$ & $4.32(3.59)$ & $t(103)=-1.96, p=.052$ \\
\hline Drug Use past month & $1.73(1.74)$ & $2.86(2.59)$ & $t(26.24)=-1.93, p=.064$ \\
\hline Drug Use past week & $1.29(1.48)$ & $2.14(2.25)$ & $t(25.98)=-1.67, p=.11$ \\
\hline
\end{tabular}

Note. For the Drug Use scores, data of three participants on smoking was missing. They were not included in the sum score analyses. Drug Use sum scores could range between 0 (never used any of the drugs) and 10 (ever used all of the drugs). Of the 10 separate drugs percentages of lifetime use are presented per genotype group. 


\section{Family History Effects}

A chi-square test showed that no differences were found between the $A$ and $G$ allele groups with respect to family history of alcoholism, $\chi^{2}(1)=0.06, p>.50$ (see Table 5.1). Only eight (of 107) participants indicated having at least one first degree family member who most probably suffered from alcohol dependence, 6 of whom were homozygous for the A allele and 2 of whom carried a $G$ allele. Owing to the small number of FHPs and the non-significant difference between the genotype groups, the outcome analyses below were not corrected for $\mathrm{FH}$. Ray and Hutchison (2004) used a more lenient criterion to assess a FHP of alcohol problems, namely: 'at least one family member with alcohol abuse.' Also if this criterion was adopted there were not significantly more FHPs in the G allele group compared with the A allele group (39\% versus $30 \%$ respectively, $\chi^{2}(1)=0.73, p=$ 0.27 , one-sided).

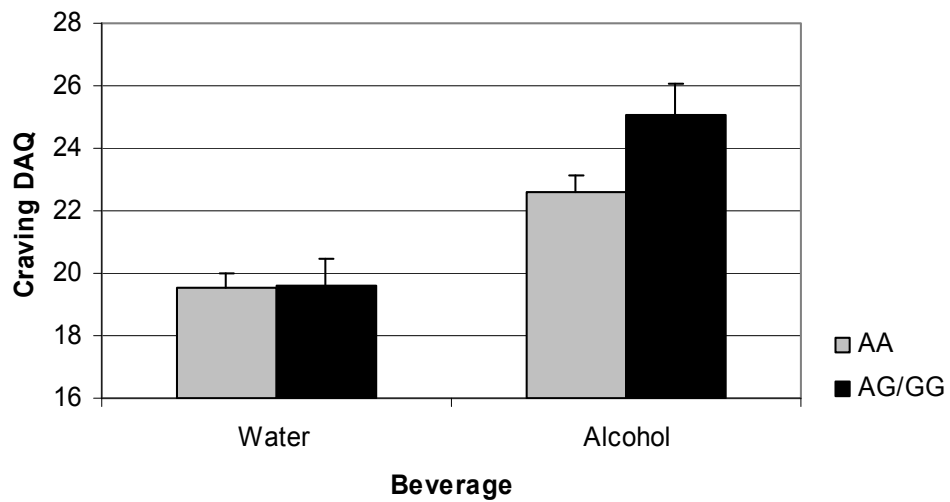

Figure 5.1. Mean scores and SE for the DAQ Craving measure during the water and alcohol exposure for both genotype groups. Participants with the $G$ allele reported significantly higher levels of craving during the alcohol trial compared with the A allele participants $(p<.05)$.

\section{Cue Reactivity Measures}

\section{Subjective Craving}

$D A Q$. The final model of craving on the DAQ did not contain any three-way interactions between beverage $\times$ genotype $x$ covariate (see Table 5.3). The withinsubjects analysis revealed a significant interaction effect of Beverage $x$ Genotype, 
such that participants with a $G$ allele were craving significantly more for alcohol after the beer exposure compared with participants homozygous for the A allele $(F(1,101)=6.10, p=.015$; see Figure 5.1). There was also a significant interaction effect of Beverage $\times$ Age, indicating that younger participants were craving more for alcohol (a larger beverage effect) than older participants $(F(1,101)=$ $6.37, p=.013)$. Since a significant Beverage $\times$ Genotype interaction was found, the between-subjects effects were analyzed per condition (water and alcohol). The effect of genotype was significant in the alcohol trial only, with $G$ allele participants reporting higher levels of craving $(F(103)=4.36, p=.039)$. The betweensubjects effects of age were also analyzed per condition, leading to a significant effect in the alcohol trial only, indicating that younger participants craved significantly more for alcohol after the beer exposure $(F(103)=4.96, p=.028)$.

Table 5.3. Within -and Between-Subjects effects for the DAQ (in $\mathrm{mm}$ ).

\begin{tabular}{|c|c|c|c|c|c|c|}
\hline \multirow[b]{2}{*}{ Predictor } & \multicolumn{3}{|c|}{ Within-Subjects Effects } & \multicolumn{3}{|c|}{ Between-Subjects Effects } \\
\hline & $\begin{array}{l}\text { Beverage effect } \\
\text { (alcohol-water) }\end{array}$ & SE & $p$ & Per Trial & SE & $p$ \\
\hline $\begin{array}{l}\text { Grand Mean } \\
\text { (intercept) }\end{array}$ & 4.24 & .47 & $<.001$ & $\begin{array}{l}\text { Water }=19.59 \\
\text { Alcohol }=23.85\end{array}$ & $\begin{array}{l}.48 \\
.57\end{array}$ & $\begin{array}{l}<.001 \\
<.001\end{array}$ \\
\hline Age & -0.47 & .19 & .013 & $\begin{array}{l}\text { Water }=-0.04 \\
\text { Alcohol }=-0.51\end{array}$ & $\begin{array}{l}.19 \\
.23\end{array}$ & $\begin{array}{l}>.50 \\
.028\end{array}$ \\
\hline Genotype & 2.36 & .95 & .015 & $\begin{array}{l}\text { Water }=0.10 \\
\text { Alcohol }=2.42\end{array}$ & $\begin{array}{l}.98 \\
1.16\end{array}$ & $\begin{array}{l}>.50 \\
.039\end{array}$ \\
\hline
\end{tabular}

Note. The Within-subjects table part, with the beverage effect (alcohol - water) as outcome, shows the significant genotype effect on the beverage effect, i.e. the genotype $\mathrm{x}$ beverage interaction. In the Between-subjects table part, effects are given separately for the alcohol and water trial. The genotype effect is significant in the alcohol trial only. The analysis was controlled for the (centered) covariates alcohol use, age, and baseline craving. Genotype effects are always AG - AA, thus, a positive Genotype effect indicates a larger effect size for the $G$ allele group. The effect of Age (in years) is the change in beverage effect if age increases with 1 year. Due to two missings on the water trial, the genotype effect on the within-subjects beverage effect is not exactly equal to the difference between the genotype effect in the alcohol trial and that in the water trial.

Actually, an additional interaction between Beverage $x$ Genotype $x$ Alcohol Use was found $(F(1,100)=4.26, p=.042)$. Given the fact that $\alpha$ was adjusted to .01 (multiple testing) this finding should be interpreted as a statistical trend and interpreted with caution and was therefore left out of the final model. To visualize this interaction, the beverage effect (alcohol - water) on the DAQ was plotted against genotype by alcohol use (median split at 38 drinks/week), adjusted for the other two covariates age and baseline craving (Figure 5.2). As can be seen, par- 
ticipants with a copy of the $G$ allele who were relatively 'light' (or moderate) heavy drinkers ( $M=29.73$ drinks/week) showed the strongest beverage effect on craving. Simple effects analysis regarding the genotype effect per level of alcohol use revealed that the $G$ allele participants showed a significantly larger beverage effect than A allele participants when alcohol use was relatively moderate $(p=.005)$. No effect of genotype was found for the heavier drinkers $(p=.41)$.

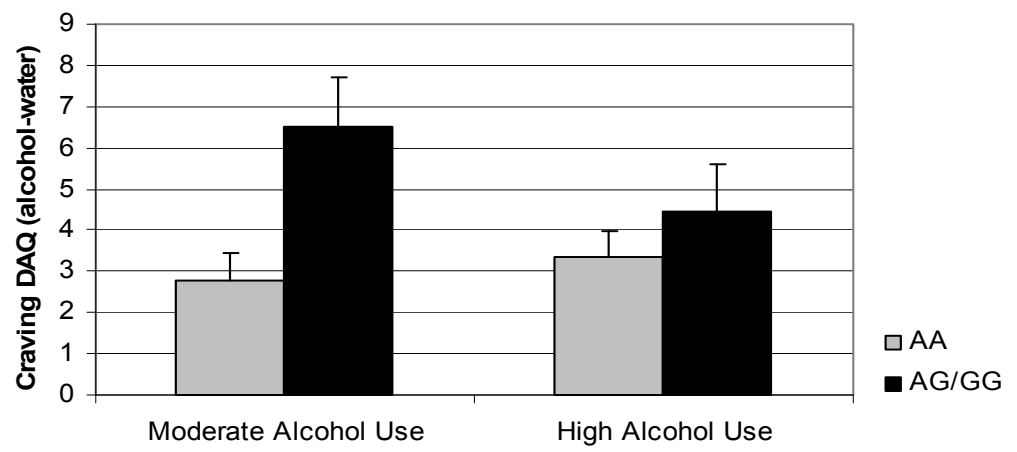

Figure 5.2. Mean scores and SE for the Beverage effect (alcohol-water) on the DAQ. A borderline significant interaction was found for Beverage $\times$ Genotype $\times$ Alcohol Use $(p=0.042, \alpha=.01)$, such that the effect of genotype on the beverage effect (= DAQ difference alcohol-water) was significant when alcohol use was relatively moderate $(p=.005)$, but not when alcohol use was high $(p=.41)$. Moderate alcohol use ranged between 10.5 - 38 drinks/week, and high alcohol use between 38.5 68.5 drinks/week. The covariates age, baseline and alcohol use, were included in their original quantitative form in all statistical analyses. Note that this covariate was dichotomized here (using a median-split) for the purpose of illustrating the interaction found.

VAS Craving Alcohol. Craving for alcohol, measured with the single VAS revealed a significant interaction effect of Beverage $\times$ Genotype $\times$ Baseline Craving $(F(1,102)$ $=8.76, p=.004$ ), such that the beverage effect (alcohol-water) was larger for $G$ allele than $A$ allele participants when baseline craving was low. The pattern was reversed when baseline craving was high (Figure 5.3, based on a median split for baseline craving). Simple effects analysis revealed that the genotype effect on the beverage effect was significant when baseline craving was low $(p=.038)$ and non-significant when baseline craving was high $(p=.21)$. Because of the significant interaction of Beverage $\times$ Genotype $\times$ Baseline craving, the between-subjects effects were analyzed per condition. On the water trial, no significant main or interaction effect was found for genotype. On the alcohol trial the interaction between Genotype x Baseline was almost significant, such that the $G$ allele participants low on baseline reported more craving for alcohol than the A allele partici- 
pants low on baseline craving $(F(1,102)=3.46, p=.066)$. The fact that the latter interaction was only borderline significant is probably due to a Type II error because of the small sample size of the $G$ allele group.

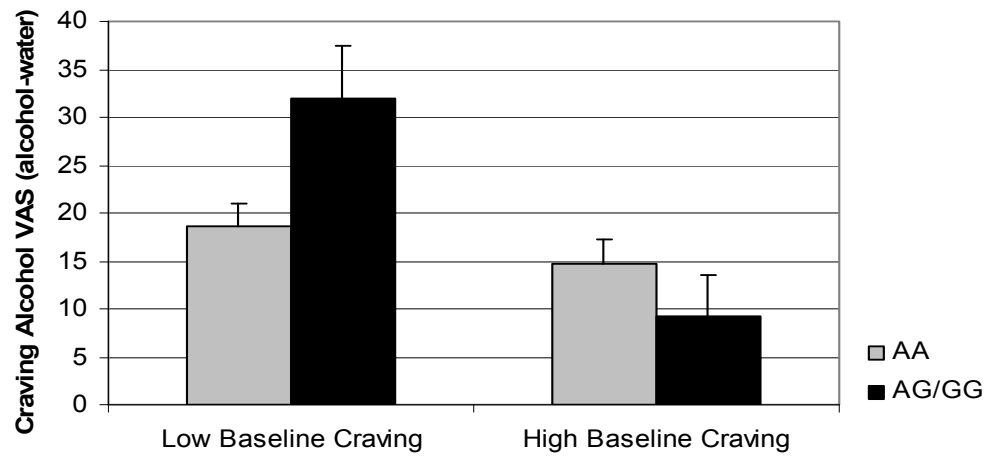

Figure 5.3. Mean scores and SE for the Beverage effect (alcohol-water) on the VAS Alcohol Craving. Analyses revealed a significant Beverage $\times$ Genotype $\times$ Baseline Craving interaction $(p<.01)$, such that participants with the $G$ allele were experiencing more craving for alcohol (a larger beverage effect) than A allele participants when baseline craving was low $(p=.038)$. The groups did not significantly differ when baseline craving was high $(p=.21)$. The covariates age, baseline and alcohol use, were included in their original quantitative form in all statistical analyses. Note that this covariate was dichotomized here (using a median-split) for the purpose of illustrating the interaction found.

VAS Craving Water. On the craving for water VAS, only a main effect of Beverage was found, such that all participants were craving significantly less for water after the alcohol exposure compared with the water exposure $(F(1,103)=17.60, p<$ $.001)$. No significant main or interaction effects were found for Genotype or any of the covariates.

\section{Mood}

Arousal. The final model for the arousal score on the mood grid did not contain any three-way interactions between beverage $x$ genotype $x$ covariate. The within subjects analysis revealed a main effect for Beverage $(F(1,103)=31.36, p<$ $.001)$. Participants experienced more arousal after the alcohol exposure compared with the water exposure. No significant main or interaction effects were found for Genotype. A trend towards a Beverage $x$ Genotype $\times$ Baseline Arousal interaction was found $(F(1,102)=6.58, p=.012 ; \alpha=.01$ to correct for multiple testing). Therefore, this interaction was removed from the final model and interpretation should be with caution. The interaction plot (see Figure 5.4, based on a median 
split for baseline arousal) shows that participants with the $G$ allele experienced a stronger Beverage effect compared with A allele participants when baseline arousal was low. The pattern was reversed when baseline arousal was high. Simple effects

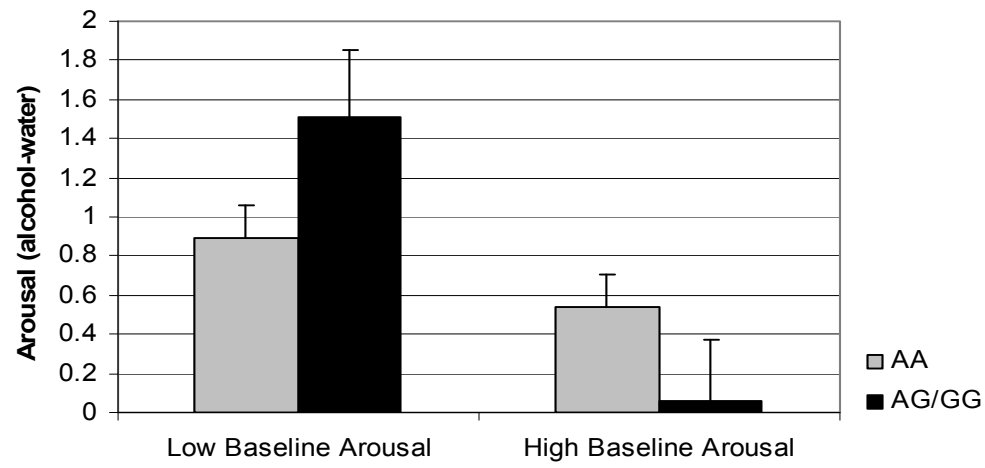

Figure 5.4. Mean scores and SE for the Beverage effect (alcohol-water) on the Arousal score on the mood grid. Analyses revealed a borderline significant Beverage $\times$ Genotype $\times$ Baseline Arousal interaction ( $p=.012, \alpha=.01)$. Simple effects showed that the effect of genotype on the beverage effect (Arousal difference alcohol-water) was non-significant, both when baseline arousal was low ( $p$ $=.13)$ and high $(p=.055)$. The covariates age, baseline and alcohol use, were included in their original quantitative form in all statistical analyses. Note that this covariate was dichotomized here (using a median-split) for the purpose of illustrating the interaction found.

analyses resulted in non-significant genotype effects on the beverage effect, both when baseline arousal was low $(p=.13)$ and high $(p=.055)$.

Valence. For the Valence dimension on the mood grid, a significant main effect for Beverage was found, such that all participants felt more positive after exposure to alcohol $(F(1,103)=19.68, p<.001)$. No significant main or interaction effects were found for Genotype or any of the covariates.

\section{Salivation}

A significant main effect of Beverage was found, indicating that participants salivated more after alcohol exposure $(F(1,103)=23.45, p<.001)$. A significant interaction between Beverage $x$ Alcohol Use revealed that the beverage effect increased with alcohol use $(F(1,103)=7.38, p=.008)$. No main or interaction effects were found for Genotype or other covariates. 


\section{Additional Analyses}

Our unequal sample size (i.e. 84 persons with $A A$ versus 24 with AG/GG genotypes) reduces statistical power and makes ANOVA more sensitive to violations of homogeneity of variance, as compared with equal sample sizes given a total $N=$ 108. To check the loss of power, we computed the smallest possible effect size that could be detected with a power of $80 \%$ or $90 \%$, respectively assuming a two-tailed $\alpha$ of $5 \%$. This smallest detectable effect size (Cohen's d) was 0.65 or 0.75 , respectively, which are both in-between medium and large effect sizes. As a protection against overlooking smaller effects, we reported the standard error of each effect in Table 5.3, allowing computation of the $95 \%$ confidence interval for each effect. Heterogeneity of variance between both groups was checked with the Levene test, for each outcome, for the difference score (alcohol - water) as well as the average of both trials. In addition, we compared the results of the t-test assuming equal variances with those of the t-test allowing unequal variances. We found no significant violations of homogeneity of variance and no contradiction between the two ttests with respect to the presence or absence of an effect of genotype.

\section{DISCUSSION}

The present study was designed to investigate whether male, nontreatment seeking, heavy drinking participants with at least one copy of the $G$ allele of the mu-opioid receptor polymorphism A118G responded differently to the exposure of alcohol cues compared to participants homozygous for the more common A allele. The main findings of the study were as follows: First, participants with a copy of the $G$ allele reported significantly more craving for alcohol on the DAQ than A allele participants (a larger beverage effect as well as more craving during the alcohol trial only). Second, a tentative interaction with alcohol use showed that mainly the moderate heavy drinkers with the $G$ allele were experiencing the strongest craving after exposure. This interaction should be considered as a statistical trend after controlling for multiple testing. Third, on the single VAS measuring craving for alcohol, an interaction with baseline craving was found, such that participants with a $G$ allele low on baseline craving showed significantly more craving for alcohol after exposure than A allele participants low on baseline craving. A similar interaction pattern for arousal on the mood grid showed a statistical trend. $G$ allele participants low on baseline arousal tended to show a larger beverage effect than $A$ allele participants low on baseline arousal, however, this simple effect was nonsignificant. Fourth, no differences were found between the two genotype groups with regard to a positive $\mathrm{FH}$ of alcoholism. Fifth, participants with a $\mathrm{G}$ allele re- 
ported having ever used drugs significantly more often, especially cannabis and amphetamines.

These findings have several implications: First, individuals with a copy of the $G$ allele seem to be more sensitive to cue-induced subjective craving for alcohol than individuals homozygous for the A allele. In the alcohol administration study by Ray and Hutchison (2004) stronger feelings of intoxication, sedation, happiness and stimulation were found in the $G$ allele group. Craving was not assessed, but the present study shows that even exposure to an alcoholic beverage increases the amount of craving for alcohol in individuals with this genetic vulnerability. Especially the $G$ allele participants still in the relatively moderate range of heavy drinking might be at risk since they showed the highest cue-induced difference scores in craving. For craving on the VAS and subjective arousal on the mood grid similar patterns were found: $G$ allele participants low on baseline craving showed a stronger beverage effect compared with A allele participants. The beverage effect scores for both genotype groups low on baseline arousal did not differ significantly but seem to point in the same direction as the craving scores. This suggests that $G$ allele individuals who are under aroused might be more inclined to use alcohol because of their stronger subjective experience after exposure. Physiologically, no indication was found for a stronger cue reactivity effect in the $G$ allele group. The $G$ allele participants did not salivate more than the participants in the A group. The finding that the heavier drinkers salivated more after alcohol exposure than the lighter drinkers is in accordance with previous research with alcohol dependent patients, in which it was found that the patients salivated more to alcohol cues than did the nonalcoholic controls (Monti et al., 1987).

Second, very few FHPs were found in the present sample. Only 6 participants in the A group (7.1\%) and 2 participants in the $G$ group (8.7\%) reported a positive family history. This was unexpected in comparison with the study of Ray and Hutchison (2004) where 25\% of the A allele participants reported having a family history of alcohol problems, versus almost $70 \%$ of the $G$ allele group participants. What could account for this difference? First, in the present study FH was operationalized as 'at least one first degree family member with alcohol dependence.' Ray and Hutchison used a more lenient criterion for FHP: 'at least one family member with alcohol abuse' (either a first or second degree relative). When applied to the present study, the more lenient criterion 'at least one family member with alcohol abuse,' did suggest a difference in FHPs between the A and $G$ allele group, but this was not significant (30\% versus $39 \%$ respectively). A second difference between the studies is that Ray and Hutchison used a prospective genotyping design (i.e. first genotyping, then selecting participants). But assuming that their selection procedure was the same for both allele groups, this cannot explain the 
difference between their and our study with respect to the association between family history and the presence of the $G$ allele. Third, both studies used student samples, which are likely to yield lower levels of FHP than community samples, because FHPs tend to drop out of school more often (Sher, 1991). One possibility could be that FHPs are less successful in the Dutch than in the American school system. Alternatively, the finding of the smaller study by Ray and Hutchison could be due to chance, although their p-value makes a type I error somewhat unlikely. It should be noted that most other studies in this area did not report family history status of the participants (e.g. Bergen et al., 1997). We are aware of one other study that reported Type 1 (late onset, often characterized by a FHN) and Type 2 (early onset, often characterized by a FHP) alcoholism, but they did not find a significant difference between the two groups with respect to the presence of the $G$ allele (Bart et al., 2005). Based on these three studies, we conclude that at present it is unclear whether the presence of the $G$ allele in the mu-opioid receptor gene is reliably related to a family history of alcoholism. More research is needed to settle this issue.

Third, we found the expected association between the $G$ allele and lifetime drug use. The $G$ allele group reported having used cannabis and amphetamines significantly more often than the A allele group. There was a trend for $G$ allele participants to have used drugs more often in the past year. These findings are in accordance with the study of Bond et al. (1998) who showed that the presence of the $G$ allele results in a more active binding of beta-endorphin to the mu-opioid receptor. However, the findings seem in contrast with the meta-analysis of Arias and colleagues (2006) who did not find an association between the $G$ allele and risk for substance dependence. A possible explanation for these differing findings could be that alcohol and substance dependence are very heterogeneous disorders, influenced by multiple genetic and environmental factors. Our study sample consisted of heavy drinking, nontreatment-seeking individuals who vary in their level of alcohol or drug (mis)use. The presence of the $G$ allele might predispose them to crave more for alcohol and experiment more with other drugs due to the rewarding effects they experience after the activation of their beta-endorphin system. Transition from habitual to compulsive use (Everitt and Robbins, 2005), however, might be in interaction with other genes and environmental factors. Also, instead of looking at a heterogeneous disorder as outcome, such as alcohol dependence, as was done in the meta-analysis by Arias and colleagues, one can also investigate subcomponents of the disorder, so-called 'endophenotypes' (Gottesman \& Gould, 2003). An endophenotype is associated with the underlying biological mechanism of the disorder, but is a more specifically defined clinical manifestation, which can be measured in a controlled fashion. Cue-elicited craving might 
prove to be a potentially useful endophenotype which could bridge part of the gap between the complex disease alcoholism and its distal, underlying genetics. Presence of the $G$ allele might contribute to a stronger cue-elicited urge to drink, but might not be related to the heterogeneous disorder overall.

Even though presence of a $G$ allele may not be a strong predictor of the development of substance dependence (Arias et al., 2006), the A118G polymorphism is an important moderator with regard to pharmacotherapeutic treatment, as has been found for smokers (Lerman et al., 2004) as well as alcoholics (Oslin et al., 2003) and possibly, in the future, for other substance use disorders. Naltrexone reduces craving and the present study showed that individuals with a copy of the $G$ allele experienced stronger craving for alcohol after exposure to an alcoholic beverage, something that will undoubtedly happen to an alcoholic patient in real life settings. If craving can be more successfully reduced in this particular subgroup of patients, the risk of relapse might be diminished as well. In the pooled dataset of Oslin and colleagues changes in craving were not reported, therefore, no conclusions can be drawn about a possible stronger decrease in craving in the $G$ allele patient group receiving naltrexone.

Some limitations of the present study should be acknowledged. First, our sample consisted of problem drinking students, most of whom met criteria for a likely diagnosis of an alcohol-problem. This is an interesting sample (sometimes labeled "non-institutionalized alcoholics") but it precludes generalizations to alcoholics in clinics. Related, FHPs are likely to be underrepresented in student samples. This could be one of the reasons why we did not find an association between FHP and the presence of the $G$ allele, although it should be stressed that on the basis of the present evidence it cannot be concluded that this relationship exists. Second, like most studies in this area (e.g. Smolka et al., 1999), we used a retrospective genetic design, i.e. participants were genotyped after they were included into the study on the basis of their excessive drinking. As expected, this strategy resulted in a much larger A allele group as compared with the $G$ allele group. An alternative for future studies could be to use a prospective genotyping design (i.e., first genotype, then select equally large groups of $A$ and $G$ allele groups by random sampling from each group). The advantage of equal sample sizes is that statistical tests of differences between both groups have more power and are more robust against heterogeneity of variance. The only study we are aware of in this area which used prospective genotyping in order to create equal groups is the study by Ray and Hutchison (2004). However, it should be noticed that they screened fewer people (76) than we included in our unselected sample (108) and ended up with a relatively small sample size. 
Future research should not only focus on participants who are already alcohol or drug dependent. It is important to identify risk genes that might predispose to heavy drinking in the first place and that might play a role in the transition from habitual to compulsive use. Since alcohol and drug dependence are polygenetic disorders, interactions between several risk genes should be investigated in order to get a better insight in their potentially enhancing effects. Identifying relevant endophenotypes, such as cue-elicited craving, could help in decomposing the complex, heterogeneous, polygenetic disease such as alcohol dependence into smaller, narrowly defined subcomponents which might be more specifically related to certain genes. This study suggests that heavy drinking individuals with a copy of the $G$ allele are more prone to experiencing cue-induced craving after exposure to an alcoholic beverage. Furthermore, they reported a higher lifetime prevalence of drug use. Future research may apply this knowledge in studies of targeted prevention and treatment of at-risk individuals.

\section{ACKNOWLEDGEMENTS}

The authors would like to acknowledge Paul Curfs for facilitating the collaboration between Experimental Psychology and the Genome Centre and Tim Schoenmakers and Marten van de Braak for collecting part of the data. This research was supported in part by a ' $\mathrm{VIDI}$ ' grant from the Dutch National Science Foundation (N.W.O.) to RWW (452.02.005). 
OPRMI POLYMORPHISM AND ALCOHOL CUE-REACTIVITY 



\section{CHAPTER 6}

\section{POLYMORPHISMS OF THE DOPAMINE D4 RECEPTOR GENE (DRD4 VNTR) AND CANNABINOID CBI RECEPTOR GENE (CNR7) ARE NOT STRONGLY RELATED TO CUE-REACTIVITY AFTER ALCOHOL EXPOSURE}

Van den Wildenberg, E., Janssen, R. G. J. H., Hutchison, K. E., van Breukelen, G. J. P., \& Wiers, R. W. (in press). Polymorphisms of the Dopamine D4 Receptor Gene (DRD4 VNTR) and Cannabinoid CB1 Receptor Gene are not Strongly Related to Cue Reactivity after Alcohol Exposure. Addiction Biology. 


\section{ABSTRACT}

Aims: Polymorphisms in the D4 dopamine receptor gene (DRD4) and the CB1 cannabinoid receptor gene (CNRT) have been associated with a differential response to alcohol after consumption. The goal of the present study was to investigate whether heavy drinkers with these polymorphisms would respond with enhanced cue reactivity after alcohol exposure.

Methods: Eighty-eight male heavy drinkers were genotyped for the DRD4 VNTR and the CNR1 rs2023239 polymorphism. Participants carrying one or two 7 (or longer) repeat alleles of the variable number of tandem repeats (VNTR) polymorphism in the DRD4 gene were classified as DRD4 L. Participants homozygous for the short allele $(<7)$ were classified as DRD4 S. Based on the presence of at least one copy of the minor C allele of the CNR1 rs2023239 polymorphism, participants were classified as belonging to either the TT homozygotes or the $\mathrm{C}$ allele carriers. Participants were exposed to water and beer in 3-minute trials. Dependent variables of main interest were subjective craving for alcohol, subjective arousal and salivary reactivity.

Results: Overall, no strong evidence was found for stronger cue reactivity (= outcome difference between beer and water trial) in the DRD4 L and CNR1 C allele groups. The DRD4 VNTR polymorphism tended to moderate salivary reactivity such that DRD4 L participants showed a larger beverage effect than the DRD4 S participants. Unexpectedly, the DRD4 L participants reported, on average, less craving for alcohol and more subjective arousal during cue exposure, compared with the DRD4 S participants. As weekly alcohol consumption increased, the CNRI C allele group tended to report more craving for alcohol during the alcohol exposure than the $T$ allele group.

Conclusions: The DRD4 and CNR1 polymorphisms do not appear to strongly moderate cue reactivity after alcohol cue exposure, in male heavy drinkers.

Key words: Alcohol, Cue Reactivity, Craving, DRD4, CNR1 


\section{INTRODUCTION}

Alcoholism continues to be one of the most prevalent psychiatric disorders. It is characterized by changes in the brain reward centre which lead to compulsive drug seeking and taking and loss of control over drinking. More or less 50-60\% in the variability for alcoholism vulnerability is determined by genes (Goldman et al., 2005). When investigating the association between a specific gene and the phenotype alcoholism, often no relationship is found (e.g. Arias et al., 2006). Given the fact that alcoholism is a heterogeneous disease which is influenced by multiple genes and environmental factors, however, this is not completely unexpected. Instead of searching for a single gene which might be important to the development of alcoholism, one can also try to identify an endophenotype and relate this to a genetic polymorphism of interest. An endophenotype is an intermediate phenotype which can be measured in a controlled fashion in the lab and which is supposed to reflect the biological underpinnings of the broader phenotype (Gottesman and Gould, 2003). Examples of endophenotypes that might distinguish between individuals who are more and less prone to developing alcohol or drug-related disorders are: the P300 amplitude reduction (e.g. Yoon et al., 2006), level of response (e.g. Schuckit and Smith, 1996), sweet preference (e.g. Kampov-Polevoy et al., 1999), changes in heart-rate (e.g. Conrod et al., 2001) and the stress response (e.g. Finn and Justus, 1997). Another possible endophenotype candidate might be cue-elicited craving (e.g. Hutchison et al., 2002a; 2002b). This study investigated the association of the D4 dopamine receptor variable number of tandem repeats polymorphism (DRD4 VNTR) and a CBI cannabinoid receptor gene polymorphism (CNR1) with cue-elicited craving, subjective arousal and saliva production after alcohol exposure. The importance of the dopamine and the cannabinoid systems in the development of alcohol and drug related disorders will be explained below.

According to Wise and Bozarth (1987) all drugs (stimulants as well as sedatives) result in an initial release of dopamine in the mesolimbic system, mainly in the nucleus accumbens. This initial activating brain response is called a psychomotor stimulant response. Robinson and Berridge (1993; 2003) report evidence that after repeated administration of the drug, this psychomotor stimulant reaction becomes sensitized, or stronger, due to adaptive changes in the brain. A release of dopamine can be generated by exposure to alcohol or drug cues and by actual drug administration (e.g. a priming dose). The dopamine release can be experienced as a craving for or "wanting" of the drug. Drug-related stimuli obtain an incentive salience; they 'pop out' because of their enhanced motivational value. Thus, the dopamine response plays an important role in the development of incen- 
tive sensitization. Several dopamine receptors have been identified, such as the D1 and D5 subtypes of the D1 family and the D2, D3 and D4 receptor subtypes of the D2 family. Both the D1 and D2 dopamine receptor families have been associated with reward (Berridge and Robinson, 1998).

Because of the important involvement of the dopamine system in reward, genetic variations, or polymorphisms, in the genes for these receptors might influence gene expression and protein levels or change the function of the protein. Thereby, they could have an impact on craving and the vulnerability for developing alcohol and drug-related disorders. Dopamine receptors are guanine (G)-protein coupled receptors. They exert their biological effects through the coupling to G-proteins which starts a signal transduction cascade in the cell. Via G-proteins, the D2 family receptors inhibit the enzyme adenylyl cyclase, which normally synthesizes cyclic AMP. The molecule cAMP is a second messenger that is responsible for the inhibition and activation of further intracellular biochemical reactions (Stoof and Kebabian, 1981). The dopamine receptor subtype we will focus on is the D4 receptor. The D4 receptor is widely distributed throughout the brain and can be found in limbic structures, which are important in incentive sensitization, such as the nucleus accumbens shell, hippocampus, amygdala and hypothalamus as well as in the frontal cortex (Schoots and Van Tol., 2003; Van Tol et al., 1991). The D4 receptor gene (DRD4) has an exon 3 polymorphism, consisting of a highly variable number of tandem repeats (VNTR). The VNTR consists of a 48 base pair (bp) which can be repeated 2 up to 11 times, in tandem (Van Tol et al., 1992). Each 48 base pair codes for a sequence of 16 amino acids in the third cytoplasmic (intracellular) loop of the receptor, a region thought to be important for G-protein coupling. The most frequently occurring variants of the DRD4 are the two (D4.2), the four (D4.4) and the seven (D4.7) repeats (Schoots and Van Tol, 2003; Van Tol et al., 1992). The different repeat sequences have been found to modulate gene expression and could differentially affect RNA stability and translational efficiency (Schoots and Van Tol., 2003). The seven repeat sequence has been found to show a blunted intracellular response to dopamine in comparison with the two and four repeat variants (Asghari et al., 1995). This blunted response to dopamine might lead to a less effective inhibition of the enzyme adenylyl cyclase which will result in higher cAMP levels. Self (1998) showed that individuals with the seven repeat allele showed elevated CAMP levels, which might enhance the dopamine signals after a priming dose or exposure to the alcohol or drug-related stimulus. Thus, individuals with the long allele might be more prone to experience craving due to their enhanced dopamine response.

Presence of the long allele ( $\geq 7$ repeats; DRD4 L) has been found to moderate urge to drink after alcohol consumption (Hutchison et al., 2002b). In a smoking 
study, the long allele was found to induce urge to smoke, subjective arousal and attention for smoking cues after smoking cigarettes (Hutchison et al., 2002a). A recent study by Shao et al. (2006) showed that in a sample of Chinese heroin addicts, cue-elicited urge to use heroin, was modulated by the presence of at least a copy of the five repeat (or longer). The seven-repeat allele has also been associated with different behavioural disorders such as ADHD and novelty seeking (Faraone et al., 2001), disorders often associated with alcohol and drug use. Previous research has shown that D2/D4 antagonists such as olanzapine and haloperidol can reduce urge to smoke (Hutchison et al., 2004), urge to drink (Hutchison et al., 2001) as well as alcohol consumption (Modell et al., 1993). Subsequent studies investigating the potentially moderating role of the DRD4 polymorphism on the effect of the D2/D4 antagonist olanzapine revealed that olanzapine reduced cueelicited alcohol craving in heavy drinkers with the DRD $4 \mathrm{~L}$ allele (Hutchison et al., 2003). In a sample of alcohol-dependent patients, the presence of at least one copy of the long allele resulted in reduced cue-induced craving and alcohol consumption (Hutchison et al., 2006). Patients with the short allele (DRD4 S) did not benefit from olanzapine treatment. This might have possible treatment implications for individuals with or without specific D4 polymorphisms.

The dopamine system is not the only neurotransmitter system which is modulated by prolonged alcohol use. The glutamatergic, GABAergic, noradrenergic, opioidergic, serotonergic and cholinergic systems have all been found to play a role in the different aspects related to the effects of alcohol (Basavarajappa and Hungund, 2002). A more recent discovery of another system involved in alcohol and drug use is the endocannabinoid signalling system. Two types of cannabinoid (CB) receptors have so far been identified, the $C B 1$ and $C B 2$. The CB1 receptor is the most prevalent G-protein-coupled receptor and is prevalent in many parts of the brain, including the brain reward system, such as the ventral tegmental area (VTA), nucleus accumbens (NAc), amygdala, hippocampus, basal ganglia, cerebellum and the (prefrontal) cortex (Basavarajappa and Hungund, 2002). The cannabinoid receptor is the main target for the psychoactive component of marijuana $\left(\Delta^{9}-\mathrm{THC}\right)$. Two endogenous cannabinoids exist that can activate the CB receptors: anandamide (AEA) and 2-arachidonylglycerol (2-AG). These endogenous cannabinoids are released from the post synaptic cell upon dopamine release in for example the VTA (Maldonado et al., 2006). Through a retrograde control mechanism these neuromodulators activate the pre-synaptic $C B 1$ receptors which in turn modulate (inhibit) the release of excitatory (glutamate) or inhibitory (GABA) neurotransmitters in the VTA and NAc. Through their inhibitory effect on the release of glutamate and GABA, the endocannabinoids have a modulating effect on DA activity in the VTA. Drugs such as alcohol, cannabis, nicotine and opioids increase 
the firing rate of DA neurons, resulting in an increase of endocannabinoids in the VTA. Activation of CB1 and DA receptors contributes to drug-seeking behaviour. Prolonged alcohol or drug use leads to heightened levels of endocannabinoids which over stimulate the $\mathrm{CB} 1$ receptors resulting in down-regulation of $\mathrm{CB} 1$ receptor function and its signal transduction (Basavarajappa et al., 1998; Hungund and Basavarajappa, 2004).

Genetic variations in the CB1 receptor gene (CNR1) have been associated with the severity of alcohol dependence (Schmidt et al., 2002) and with polysubstance abuse (Comings et al., 1997; Zhang et al., 2004). Zhang et al. identified 3 additional exons in the CNRT gene located $5^{\prime}$ of the coding exon 4. The CNRT SNP rs2023239 is a T to C substitution at the $3^{\prime}$ end of intron 2 close to the secondary promoter and alternate transcription initiation site close to exon 3. This SNP seems to result in an increased mRNA expression (thus increased CBI receptor expression) in several brain areas, due to a different transcription of the DNA. Unpublished data from Hutchison and colleagues showed that the rs2023239 polymorphism of the CNR1 was associated with a greater craving for cannabis, more alcohol, cannabis and tobacco use, stronger subjective positive reactions to alcohol (both after oral administration, see Haughey et al., 2005; and after infusion), and with a stronger positive affective response to smoking. A neuroimaging study with heavy drinkers revealed that individuals with the $C$ allele demonstrated more activation in the bilateral NAc and bilateral VTA than individuals with the TT genotype, both during an alcohol cue and (even enhanced) after a priming dose of alcohol. Furthermore, it seems that the CNRI SNP moderates the effect of the DA antagonist olanzapine, both in alcohol dependent and non-dependent drinkers. Individuals carrying a copy of the rare C allele reported a decrease in craving and alcohol consumption while on olanzapine compared with placebo. A recent association study failed to replicate the findings of Zhang et al. of an association between the rs2023239 SNP and alcohol or substance dependence (Herman et al., 2006). Also, a small study by Pierucci-Lagha and colleagues (2006) failed to find stronger subjective effects after alcohol consumption in $\mathrm{C}$ allele heavy drinkers. In fact, the $C$ allele participants showed reduced subjective effects compared with the homozygous $T$ allele participants. However, no strong conclusions can be drawn from this study since the sample size was relatively small.

The involvement of the endocannabinoid system in addictive behaviours has implications for pharmacotherapeutic treatment. Animal research has shown that CB1 agonists increase motivation for alcohol intake (Gallate et al, 1999) while CB1 antagonists result in a decreased alcohol intake (for a review see Le Foll and Goldberg, 2005). CB1 receptor knockout mice demonstrate a strong reduction in voluntary alcohol consumption and show a complete lack of alcohol-induced DA 
release in the nucleus accumbens (Hungund et al., 2003). Also in humans, preclinical and clinical studies with the $\mathrm{CB} 1$ receptor antagonist rimonabant suggest that blocking the $\mathrm{CB} 1$ receptor might be a potentially efficacious new drug treatment (Le Foll and Goldberg, 2005).

The present study was aimed at investigating whether participants carrying at least one 7 repeat allele or longer (DRD4 L) of the DRD4 VNTR showed more cue reactivity (subjective craving, subjective arousal and salivary reactivity) after alcohol cue exposure compared with the short allele participants (DRD4 S). With respect to the CNR1 rs2023239 polymorphism, more cue reactivity was expected for the participants carrying at least one copy of the minor $C$ allele compared with those homozygous for the common $\mathrm{T}$ allele. Furthermore, we expected that the DRD4 L and CNRT C allele group would report significantly more alcohol and drug use than the DRD4 S and CNRT T allele groups, respectively. Finally, given mixed findings of the relationship between the DRD4 polymorphism and novelty seeking (Kluger et al., 2002), we investigated whether there were any differences between the long and short allele groups with respect to a related concept, reward sensitivity, as measured with the Behavioural Activation System (BAS) scales (Carver and White, 1994).

\section{MATERIALS AND METHODS}

\section{Participants}

Participants were 88 male heavy drinkers who had previously participated in the cue-reactivity study, described below, in relation to the A1 18G polymorphism of the mu-opioid receptor gene (OPRM1; results described elsewhere). Of the 109 participants who took part in the original study, 88 provided a second written informed consent for additional DNA analyses of the DRD4 and CNR1 polymorphisms. Participants were paid $€ 15$ for taking part in the cue exposure experiment. They did not receive an additional monetary reward for their informed consent for the additional DNA analyses. The study was approved of by the Medical Ethics Committee of the Academic Hospital in Maastricht. The A1 18G polymorphism of the OPRM1 gene was not confounded with the polymorphisms of the DRD4 VNTR $(r=-.06)$ or CNRT SNP $(r=.01)$.

\section{Materials and Measures}

\section{Pre-test Measures}

Alcohol Use. Alcohol use was measured with a self-report questionnaire (Wiers ef al., 1997) based on the Time-Line Follow-Back method (Sobell and Sobell, 1990). 
Alcohol consumption for each day of the week prior to the experiment was filled out and the participants indicated whether this was more, less or equal to what they would normally drink on that day of the week. A standard drink contains $10 \mathrm{~g}$ alcohol. Furthermore, the number of binges ( 6 drinks or more on one occasion) of the past two weeks was reported.

Alcohol-related Problems. The 10-item Alcohol Use Disorder Identification Test (AUDIT; Saunders et al., 1993) was used to measure alcohol-related problems. The questionnaire is divided into two subscales: items 1-3 measure alcohol consumption and item 4-10 alcohol-related problems.

Drug Use. The participants were asked to report the lifetime use of ten other drugs: Cigarettes, Cannabis, Amphetamines/Speed, XTC/MDMA, Cocaine, Hallucinogenic drugs (e.g. magic mushrooms, smartdrugs, LSD), Heroin, Medicines/Pills (e.g. sedatives, benzodiazepines), other Opiates/Analgesics (e.g. methadone) and Inhalants (e.g. laughing gas, poppers). Based upon the question if the participant had ever used any of the 10 different drugs, a sum score was calculated ranging from 0 (never used any of the drugs) to 10 (ever used all of the 10 drugs).

BISBAS Scales. In order to measure sensitivity to reward and punishment, the Dutch translation (Franken, 2002) of the behavioural activation/approach system (BAS) and behavioural inhibition system (BIS) scales was administered (Carver and White, 1994). The BIS/BAS questionnaire consists of 24 items divided into 4 subscales. The BIS scale (7 items) measures reactions to the anticipation of punishment (e.g. "I worry about making mistakes"). Furthermore, 3 BAS subscales are included. The BAS Reward Responsiveness scale measures the positive reaction to the occurrence or anticipation of reward (e.g. "When I get something I want, I feel excited and energized"). The BAS Drive items reflect the pursuit of desired goals (e.g. "I go out of my way to get things I want"). The BAS Fun Seeking scale consists of items which focus on the willingness to impulsively approach a potentially rewarding event (e.g. "I crave excitement and new situations").

\section{Cue Reactivity Measures}

Craving for Alcohol. Craving for alcohol was assessed by means of a $100 \mathrm{~mm}$ Visual Analogue Scale (VAS) ranging from "absolutely no urge" to "an irresistible urge" to drink. A second craving measure was the Dutch translation (Franken ef al., 2003) of the Desire for Alcohol Questionnaire (DAQ; Love et al., 1998). The 14 items assess the desire to drink alcohol at the moment of testing itself. The DAQ is composed of four subscales: (1) Strong Desires and intentions to drink; (2) 
Negative Reinforcement; (3) Control over drinking and (4) Mild Desires to drink. As response format, $70 \mathrm{~mm}$ VAS were used with the anchors "strongly disagree" and "strongly agree". The mean score across all 14 items was used as outcome measure.

Arousal. The affect grid (Russell et al., 1989) was used to assess subjective arousal. The mood grid is a square of $9 \times 9$ consisting of two dimensions: a valence dimension ranging from 'unpleasant feelings' (left side of the grid) to 'pleasant feelings' (right side) and an arousal dimension which ranged from 'sleepiness' (bottom) to 'high arousal' (top). The participant was asked to place an $X$ in one of the 81 possible positions. Affect descriptors were placed at the corners and sides of the grid to elucidate which mood state belonged to which part of the grid. Based on the position of the $X$, two scores could be computed (valence and arousal), both ranging from 1 to 9 . Only the arousal score was used here.

Salivary Reactivity. The salivation measure was based on the Strongin, Hinsie and Peck technique (in White, 1977). Participants placed three cotton rolls in the mouth: one under the tongue and two between the inner cheek and lower gum, on each side of the mouth. Weight of the cotton rolls (in milligrams) was determined before and after the 3-min cue exposure trial. The amount of saliva that was produced during cue exposure was indicated by the weight difference. To prevent loss of saliva, the cotton rolls were put in sealed, small-sized plastic bags.

\section{Genotyping}

DRD4. The 48 bp VNTR in Exon 3 of the DRD4 was assayed using a modification (Anchordoquy et al, 2003) of the method of Sander et al. (1997). The primer sequences (Lichter et al.,1993) were: forward, 5'-AGGACCCTCATGGCCTTG-3' (fluorescently labelled), and reverse, 5'-GCGACTACGTGGTCTACTCG-3'. This method results in PCR product of (in bp): 379, 427, 475 (4 repeats), 523, 571, 619 (7 repeats), 667, 715, 763, and 811 . The genotyping success rate was 99\%. One sample failed. The allele and genotype frequencies for the remaining 87 participants are presented in Table 6.1. Allele frequencies demonstrated HardyWeinberg equilibrium and are consistent with other studies (e.g. Lerman et al., 1998). Participants were classified as DRD4 S (both alleles $<7$ repeats; S/S) or DRD4 $\mathrm{L}$ (homozygous or heterozygous for an allele $\geq 7$ repeats; $\mathrm{L} / \mathrm{L}$ or $\mathrm{S} / \mathrm{L}$ ). This was based on other studies grouping short and long allele participants (e.g. Lerman et al., 1998) and based on molecular studies suggesting a different D4 receptor function for the seven repeat allele (Asghari et al., 1995). One participant was homozygous for the 7 repeat allele. 
CHAPTER 6

Table 6.1. Allele and Genotype Frequencies for the DRD4 VNTR polymorphism ( $n=87)$.

\begin{tabular}{|c|c|c|}
\hline Allele/Genotype & Number & Frequency (\%) \\
\hline \multicolumn{3}{|l|}{ Allele } \\
\hline 2 & 15 & 8.62 \\
\hline 3 & 12 & 6.90 \\
\hline 4 & 119 & 68.39 \\
\hline 5 & 3 & 1.72 \\
\hline 6 & 0 & 0.00 \\
\hline 7 & 23 & 13.22 \\
\hline 8 & 2 & 1.15 \\
\hline Total & 174 & 100.00 \\
\hline \multicolumn{3}{|l|}{ Genotype } \\
\hline $2 / 2$ & 1 & 1.15 \\
\hline $2 / 4$ & 9 & 10.34 \\
\hline $3 / 4$ & 7 & 8.05 \\
\hline $3 / 5$ & 1 & 1.15 \\
\hline $2 / 7$ & 4 & 4.60 \\
\hline $3 / 7$ & 3 & 3.45 \\
\hline $3 / 8$ & 1 & 1.15 \\
\hline $4 / 4$ & 43 & 49.43 \\
\hline $4 / 5$ & 2 & 2.30 \\
\hline $4 / 7$ & 14 & 16.09 \\
\hline $4 / 8$ & 1 & 1.15 \\
\hline $7 / 7$ & 1 & 1.15 \\
\hline Total & 87 & 100.01 \\
\hline \multicolumn{3}{|c|}{ Genotype Classification } \\
\hline Short (< 7 Repeats) & 63 & 72.41 \\
\hline Long ( $\geq 7$ Repeats) & 24 & 27.59 \\
\hline Total & 87 & 100.00 \\
\hline
\end{tabular}


CNR1. Genomic DNA was isolated from from buccal cells (2 Omniswabs per sample, Whatman, The Netherlands) with the QIAamp DNA Mini Kit (QIAgen, The Netherlands). For both procedures the manufacturers protocols were followed. DNA concentration and purity were measured with a Nanodrop spectrophotometer. Determination of the CNR1 rs2023239 SNP genotype was performed by Taqman ${ }^{\circledR}$ SNP genotyping assays. PCR primers and Minor Groove Binding (MGB) allele specific probes were ordered from Applied Biosystems (assay ID: C_1 1600616). Amplification and detection were carried out in duplicate in $384 \mathrm{w}$ plates on an ABI7900HT Real-time PCR system according to the manufacturers procedures, followed by automatic genotype analysis using the Sequence Detection Software $\vee 2.3$ (Applied Biosystems, The Netherlands). Three participants were genotyped with the CC combination (3.41\%), 24 were heterozygous (CT; 27.27\%) and 61 were homozygous for the common T allele (TT; 69.32\%). Allele frequencies demonstrated Hardy-Weinberg equilibrium. Allele frequency was .17 for the $C$ allele and .83 for the T allele. In the analyses the CC and CT individuals were grouped.

\section{Procedure}

A maximum of 5 participants was tested simultaneously, at individual tables, separated from each other by screens. The beverages were hidden under two inverted, opaque, tinfoil containers that were placed in front of the participant. The container on the left side always contained an empty glass and a bottle of commercially labelled spring water ("Spa Blauw"). The container on the right side always contained an empty beer glass and a bottle of "Brand" beer (all participants had previously indicated that beer was their favourite alcoholic beverage). Before the start of the cue-exposure trials, baseline measures of subjective craving, subjective arousal and saliva production were taken. The participants were instructed that it was important to perform all the actions pointed out by the experimentor simultaneously.

The cue exposure started with a 3-min relaxation period, based on Monti et al. (1987) and Rohsenow et al. (2000). Next, the water and alcohol exposure trials followed. To prevent transfer effects the water exposure always came first and the alcohol exposure second (Monti et al., 1987). Participants were asked to place the three cotton rolls in their mouth in the way previously described to them: one sublingually and two buccally. Subsequently, they were asked to lift the container on the left, take the empty glass and fill it up half with water from the bottle. After this, the 3 minutes of exposure started. Participants were asked to raise the glass, to carefully look at it and sniff the contents for 5 seconds. This procedure was re- 
peated 15 times in total. After 3 min they were asked to put down the glass and cover it with the container. Next, they removed the dental rolls, put them back in the plastic bag and sealed it well to prevent loss of saliva. To prevent a dry mouth from the cotton rolls they were given a cup of water to take a sip. Then, the participants filled out the Affect Grid to measure subjective arousal, and the VAS and $D A Q$ to measure craving for alcohol. After the questionnaires had been returned to the experimentor, the same procedure was repeated for the alcoholic beverage under the right container. After the cue exposure, the participants received the BISBAS questionnaire.

The final part of the experiment consisted of the collection of buccal cells with several omni swabs in order to determine the genotype. The Drug use questionnaire was filled out on the next day, when they returned for another part of the experiment.

\section{Outcome Analyses}

The study design was a 2 (Beverage: Water vs Alcohol) $\times 2$ (Genotype: S vs L (DRD4) or TT vs CT/CC (CNR7)) factorial design with between-subject covariates alcohol use and baseline of the outcome at hand, and was analyzed accordingly, using repeated measures ANOVA (SPSS version 12.0.1). Age was not entered in the analyses as a covariate since no differences were found between the groups. As can be seen in Table 6.2, the power for testing a possible (though not hypothesized) DRD4 x CNR1 interaction was too low (only 7 participants were classified as having the DRD4 L and CNR1 C allele). The correlation between both factors was .01 , therefore, no indication of confounding between the two genotypes was found. For each of the 4 outcome variables ( 2 for subjective craving, 1 for arousal and 1 for saliva) the initial model included interactions of genotype with each covariate. Each covariate was first centered by subtracting the total sample mean from it, to prevent possible collinearity and to allow interpretation of the withinsubject effect as beverage effect (alcohol-water) for the average person in the sample, and interpretation of genotype $x$ beverage interaction as interaction for persons scoring average on covariates.

Table 6.2. Crosstabs for the DRD4 and CNR1 polymorphisms ( $n=87)$.

\begin{tabular}{l|l|l|l}
\hline & DRD4 & & \\
\cline { 2 - 4 } CNRT & SS & SL/LL & 61 \\
\hline TT & 44 & 17 & 26 \\
\hline $\mathrm{CT} / \mathrm{CC}$ & 19 & 7 & 87 \\
\hline Total & 63 & 24 & 6 \\
\hline
\end{tabular}


As our main interest was in effects of beverage and genotype, our main focus was on the within-subject ANOVA part. This comes down to an ANCOVA with the beverage effect (outcome difference between alcohol and water) as dependent variable, which is our measure of cue reactivity, and genotype and covariates as independent variables. Genotype x covariate interactions were deleted from this model if non-significant, using $\alpha=.01$ to correct for multiple testing. If the final model for the within-subject ANOVA part did not contain any beverage $x$ genotype $x$ covariate interaction and the beverage $x$ genotype interaction was not significant, using $\alpha=.05$, then the between-subject ANOVA part was considered. This comes down to an ANCOVA with the average of both conditions (water and alcohol) as dependent variable. If a beverage $x$ genotype ( $x$ covariate) interaction was found, then a separate ANCOVA was run per condition (water, alcohol). In both cases; that is, ANOVA of the average of both conditions, and ANOVA per condition, nonsignificant genotype $x$ covariate interaction was removed from the model before testing main effects ${ }^{3}$.

\section{RESULTS}

\section{Pre-test Measures}

\section{Alcohol and Drug Use}

As can be seen in Table 6.3, regarding weekly alcohol use, number of binges and alcohol-related problems no significant differences were found between the DRD4 $S$ and $L$ participants or between the $C$ and $T$ allele participants on the CNRT. Also, lifetime drug use did not reveal any differences between the genotype groups.

\section{BISBAS}

The DRD4 L participants displayed a higher BAS Reward Sensitivity compared with the DRD4 S participants, $t(85)=-2.44, p=.017$ (see Table 6.3). However, this effect was no longer significant after multiple testing. Including the BAS Reward score as a covariate in the analyses did not lead to different results. No differences between DRD4 S and DRD4 L participants were found on the other subscales.

\footnotetext{
${ }^{3}$ Since the participants were tested in groups with a maximum of 5 people, all analyses were repeated with the centered covariate Block (to test the lineair effect of testing in groups) and the square of the variable Block (quadratic effect). None of these analyses revealed any significant effects. Therefore, these covariates were left out of the final analyses.
} 


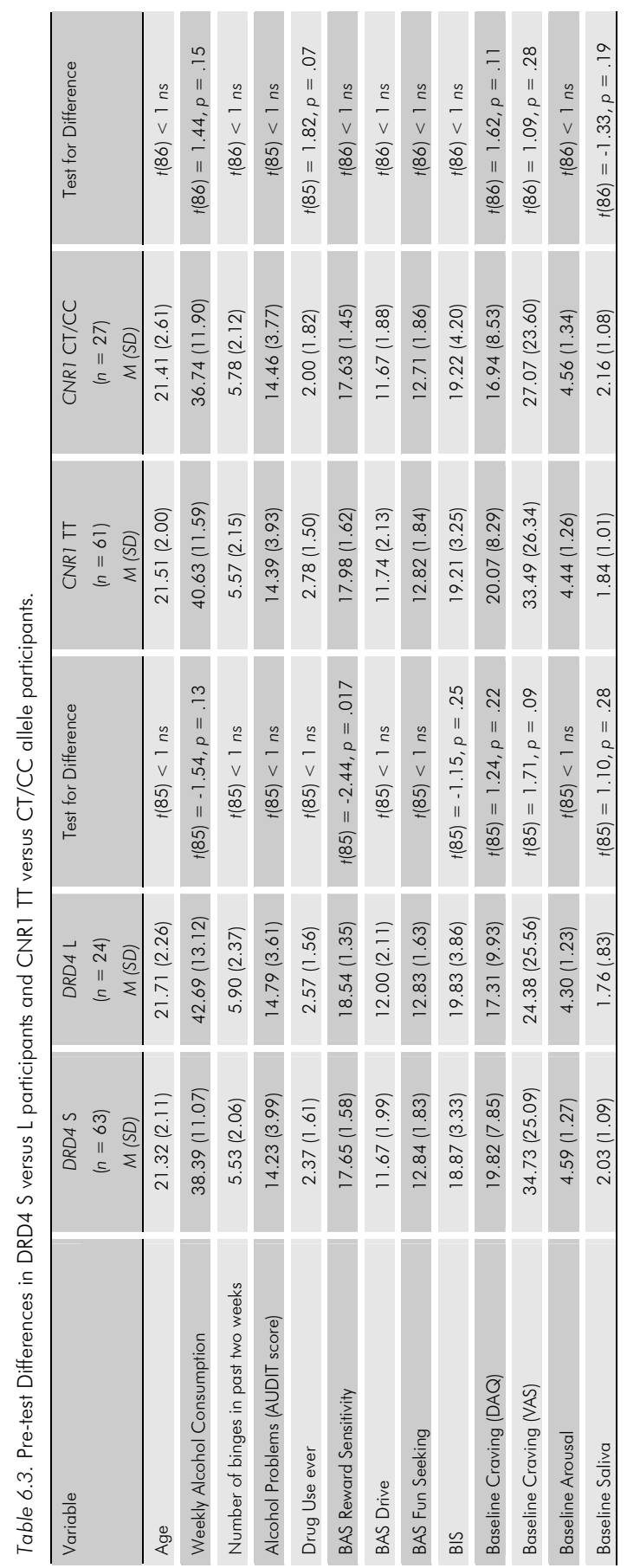




\section{DRD4 Cue Reactivity Effects}

\section{Subjective Craving}

VAS Craving. Within-subjects, a main effect of beverage was found, $F(1,83)=$ 68.50, $p<.001$, indicating that participants were craving more for alcohol after the beer exposure. No significant effects of genotype were found in the withinsubjects ANOVA, neither genotype by beverage, nor genotype by beverage by covariate. The between-subjects ANOVA showed a significant main effect of DRD4, such that on average over both trials, the DRD4 $\mathrm{S}$ group was craving more for alcohol than the DRD4 $\mathrm{L}$ group, $F(1,83)=8.23, p=.005$. Average craving of both exposure trials for the $S$ group was $43.91 \mathrm{~mm}$ ( $S E=1.37$ ), versus $36.21 \mathrm{~mm}$ (SE $=2.26)$ for the $L$ group.

$D A Q$. For craving on the DAQ, a main effect of beverage was found such that participants craved significantly more for alcohol after the beer exposure, $F(1,79)$ $=48.83, p<.001$. No effects of genotype were found.

\section{Arousal}

Within-subjects, a main effect of beverage was found, such that participants became more aroused after exposure to beer, $F(1,83)=29.00, p<.001$. No significant effects were found of genotype. The between-subjects ANOVA revealed a significant main effect of genotype such that the DRD $4 \mathrm{~L}$ participants were on average more aroused during cue exposure compared with the DRD4 S participants, $F(1,83)=6.20, p=.015$. The average arousal score for the $S$ group was 4.73 $(S E=.11)$ versus $5.26(S E=.18)$ for the $L$ group.

\section{Salivary Reactivity}

The within-subject analysis revealed a borderline significant Beverage $\times$ Genotype interaction, such that individuals with the DRD4 $\mathrm{L}$ allele tended to show a larger beverage effect than the DRD4 $\mathrm{S}$ individuals, $F(1,83)=3.41, p=.068$ (Figure 6.1). Separate between-subjects effects per condition (water or alcohol) did not reveal any significant effects of genotype. 


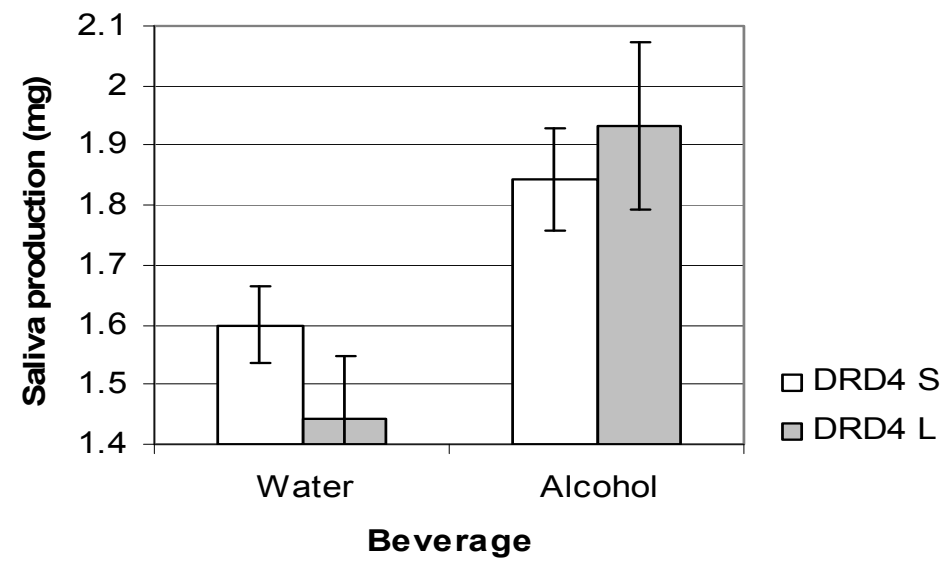

Figure 6.1. Mean scores and SE for Saliva production during the water and alcohol exposure for both DRD4 short (S) and long (L) allele groups. Participants with the DRD4 $\mathrm{L}$ allele reported a borderline significant larger beverage effect (alcohol-water) compared with the DRD4 S allele participants ( $p=$ .068). Separate between-subjects effects per condition (water or alcohol) did not reveal any significant effects of genotype.

\section{CNR1 Cue Reactivity Effects}

\section{Subjective Craving}

VAS Craving. No significant main or interaction effects were found of genotype.

$D A Q$. The within-subjects analysis on the $D A Q$ revealed a borderline significant three-way interaction between Beverage $\times$ CNR1 $\times$ Alcohol Use, $F(1,81)=5.75, p$ $=.019$ ( $\alpha=.01$, multiple testing), such that participants with a $C$ allele showed $a$ larger beverage effect on craving as weekly alcohol consumption increased. For the T allele participants, this pattern was reversed. In order to easily plot the interaction (see Figure 6.2), the covariate alcohol use was dichotomized as relatively low ("moderate" users) versus high, based on a median split (39 drinks/week).

Simple effects analysis revealed that the genotype effect on the beverage effect was significant only when alcohol use was relatively high, $F(1,40)=4.78, p=$ .041 ), with the $C$ allele participants showing a significantly larger beverage effect than the T allele participants. Separate between-subjects effects per condition (water and alcohol) revealed that the interaction between CNR $1 \times$ Alcohol Use was borderline significant in the alcohol trial only, $F(1,83)=3.79, p=.055$. Thus, as 
alcohol consumption increased, the $\mathrm{C}$ allele group tended to crave more for alcohol during the alcohol exposure than the TT group. No within or between subjects main effect was found for genotype.

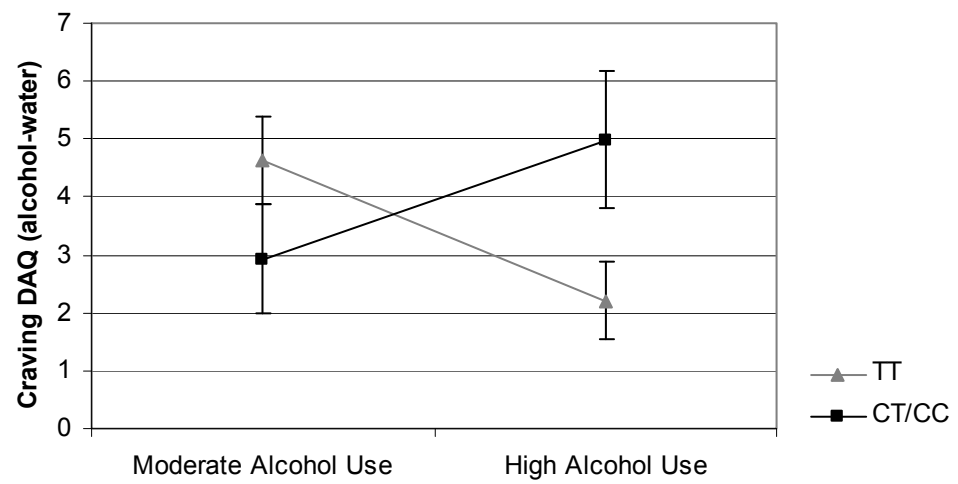

Figure 6.2. Mean scores and SE for the Beverage effect (alcohol-water) on the DAQ. A borderline significant interaction was found between Beverage $\times$ CNR $1 \times$ Alcohol Use $(p=.019, \alpha=$ $.01)$, such that individuals with a copy of the $C$ allele tended to show a larger beverage effect (alcohol-water) on craving as alcohol use increased. Simple effects analysis revealed that the effect of genotype was significant when alcohol use was high $(p=.041)$, but not when alcohol use was relatively moderate $(p=.23)$.

\section{Arousal}

No significant main or interaction effects were found of Genotype.

\section{Salivary Reactivity}

Within-subjects, a borderline significant three-way interaction between Beverage $x$ CNR1 x Baseline saliva revealed that $C$ allele participants relatively low on baseline saliva showed a stronger beverage effect than $T$ allele participants low on baseline, $F(1,83)=6.70, p=.011$ ( $\alpha=.01$, multiple testing). In order to easily plot the interaction (see Figure 6.3), the covariate baseline saliva was dichotomized as low versus high, based on a median split. 
Simple effects analysis revealed that the genotype effect on the beverage effect was significant only when baseline salivary production was relatively low, $F(1,41)$ $=7.62, p=.009$ ), with the $C$ allele participants showing a significantly larger beverage effect than the T allele participants. Separate between-subjects effects per condition (water and alcohol) revealed that the CNR1 $x$ Baseline saliva interaction was significant in the alcohol trial only, $F(1,83)=5.48, p=.022$. Thus, $C$ allele participants low on baseline saliva responded with significantly more salivary reactivity during the alcohol exposure compared with T allele participants low on baseline saliva production.

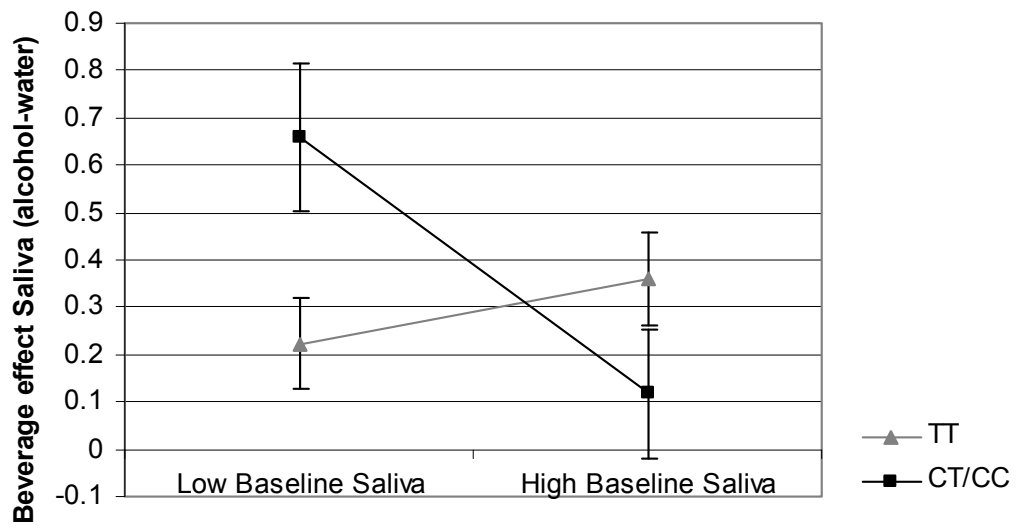

Figure 6.3. Mean scores and SE for the Beverage effect (alcohol-water) of Saliva. A borderline significant interaction was found between Beverage $\times$ CNR1 $\times$ Baseline Saliva $(p=.011, \alpha=.01)$. Simple effects analysis revealed that the effect of genotype on the beverage effect was significant when baseline saliva production was low ( $p=.009)$, and non-significant when baseline saliva production was high $(p=.25)$.

\section{Effect size}

In order to investigate whether the lack of strong genotype effects on the beverage effect (within-subjects) for the DRD4 VNTR as well as the CNRI polymorphism were due to a power problem, we calculated the $95 \%$ confidence intervals and Cohen's effect size $d$, for the genotype effect on the beverage effect for each outcome (Cohen, 1992). Also, confidence intervals and effect sizes for the genotype main effect (between-subjects) are shown. As can be seen in Table 6.4 most withinsubjects effect sizes were rather small. For the DRD4 genotype, a small to medium effect size was found for saliva $(d=.44)$. Thus, the effect of the DRD4 polymorphism on saliva reactivity would have been significant if 41 participants per geno- 
type subgroup (DRD4 S versus DRD4 L) had been tested (Cohen, 1992), assuming the present $d$ to be the true population $d$, of course. Our sample consisted of 63 DRD4 S allele participants versus 24 participants in the DRD $4 \mathrm{~L}$ group.

Table 6.4. 95\% Confidence Intervals (CI) and Effect sizes for both the DRD4 and CNR1 Genotype effects on the Within-Subjects Beverage effect (alcohol-water) and on the Between-Subjects Average effect ((water + alcohol)/2) of the 4 dependent variables.

\begin{tabular}{|c|c|c|c|c|c|c|}
\hline & \multicolumn{3}{|c|}{ DRD4 } & \multicolumn{3}{|c|}{ CNRI } \\
\hline & \multicolumn{2}{|c|}{$95 \% \mathrm{Cl}$} & \multirow[t]{2}{*}{ Effect Size (d) } & \multicolumn{2}{|c|}{$95 \% \mathrm{Cl}$} & \multirow[t]{2}{*}{ Effect Size (d) } \\
\hline & Lower & Upper & & Lower & Upper & \\
\hline \multicolumn{7}{|c|}{ 1. VAS Craving } \\
\hline WS & -4.76 & 10.93 & .19 & -2.89 & 11.77 & .28 \\
\hline BS & 2.36 & 13.05 & .69 & -3.29 & 7.19 & .17 \\
\hline \multicolumn{7}{|c|}{ 2. DAQ } \\
\hline WS & -1.49 & 2.38 & .11 & -2.16 & 1.53 & .08 \\
\hline BS & -1.19 & 3.00 & .21 & -0.42 & 3.58 & .36 \\
\hline \multicolumn{7}{|c|}{ 3. Arousal } \\
\hline WS & -0.40 & 0.73 & .14 & -0.64 & 0.44 & .09 \\
\hline BS & 0.11 & 0.97 & .60 & -0.14 & 0.70 & .31 \\
\hline \multicolumn{7}{|c|}{ 4. Saliva } \\
\hline WS & -0.02 & 0.51 & .44 & -0.33 & 0.19 & .12 \\
\hline BS & -0.23 & 0.29 & .06 & -0.13 & 0.36 & .22 \\
\hline
\end{tabular}

Note. Effect sizes (Cohen's d; as computed from Mean difference/SE * $\sqrt{ }(1 / \mathrm{N} 1+1 / \mathrm{N} 2)$ adjusted for covariates) are based on the same final model as $95 \% \mathrm{Cl}$, i.e. with covariates (alcohol use and baseline of the outcome at hand) and without three-way interactions.

\section{DISCUSSION}

The present study was designed to investigate whether non-treatment seeking, heavy drinking male individuals carrying a copy of the DRD4 L allele of the D4 receptor gene or a copy of the CNRI C allele of the CBI receptor gene would respond with more cue-reactivity after alcohol exposure, compared with participants homozygous for the major allele (DRD4 S or CNR1 T respectively). Overall, no strong evidence in support of the hypothesized associations was found. First, no significant effect of genotype was found on cue reactivity, i.e. on the beverage effect (alcohol-water), for either the DRD4 VNTR or the CNRI SNP on any of the dependent variables (subjective craving, subjective arousal and salivary reactivity). One borderline significant within-subjects main effect was found for the DRD4 genotype on saliva. The DRD4 $\mathrm{L}$ group tended to show a larger beverage effect than the DRD4 S group. Second, between-subjects, two significant main effects were found for the DRD4 genotype. Contrary to what would be expected, the 
DRD4 S group reported a higher average craving for alcohol on the VAS compared with the DRD4 $\mathrm{L}$ group. With respect to subjective arousal, the DRD4 L group reported feeling more aroused on average than the DRD4 S group. Third, after correcting for multiple testing, two potentially interesting borderline significant interactions were found for the CNR1 genotype. As weekly alcohol consumption increased, the $C$ allele participants showed a larger beverage effect on the DAQ (due to more craving during the alcohol exposure) than the $T$ allele participants. Furthermore, it seemed that $C$ allele participants low on baseline saliva production showed a larger beverage effect (due to a higher salivary reactivity after alcohol exposure) compared with the T allele participants low on baseline saliva. Fourth, contrary to what was expected, no differences were found between DRD4 S and DRD4 $\mathrm{L}$ participants or between $\mathrm{C}$ and $\mathrm{T}$ allele participants with respect to alcohol or drug use. Finally, the DRD4 L participants were significantly more sensitive to reward than the DRD4 S participants as shown by a higher score on the BAS Reward subscale. Perhaps the DRD4 L allele is more related to reward sensitivity than to the related concept of novelty seeking, but note that for this trait the initial positive associations were also not replicated (Kluger et al., 2002). Hence, replication of this positive finding is necessary.

The lack of strong effects of genotype might be due to several factors. In previous studies the participants were subjected to actual alcohol consumption or the smoking of a cigarette (e.g Hutchison et al., 2002a, 2002b). Possibly, in the present study, stronger differences between the genotype groups would have been found if the participants had received a "priming dose" of the alcoholic beverage instead of being exposed to the cues only. Also, stronger effects might be found in alcohol-dependent patients, because they usually respond with stronger cuereactivity after exposure to an alcoholic beverage. Another possible explanation could be a lack of power to find differences between the genotype subgroups due to a relatively small sample size. In order to investigate this possibility we calculated confidence intervals and effect sizes (Cohen's $d$ ) of all within and between-subjects effects of genotype. A small to medium effect size was found for the DRD4 VNTR on the beverage effect of saliva. If our DRD4 $\mathrm{L}$ group had been somewhat larger (41 instead of 24 participants) a significant difference between the two groups would have been found (assuming our sample effect size to equal the population effect size), with the DRD4 L allele group showing a larger beverage effect on salivary reactivity than the DRD4 $\mathrm{S}$ allele group. Thus, it seems that the DRD4 VNTR moderates salivary responding to alcohol cues. The lack of a significant beverage $x$ genotype effect on craving (either VAS or DAQ) for the DRD4 as well as the CNRT genotype cannot be explained by a lack of power as becomes clear from the very small effect sizes found for these 2 craving measures (ranging from .08 to .28, see 
Table 6.4). In order to find a significant difference in craving between the genotype subgroups these would have to consist of at least 100 participants per group (with $d=.28$ ) and even more with the smaller effect sizes. In case of such small effect sizes requiring many participants to find a difference, one might question the clinical relevance of this difference. It should be noted that also in the study by Hutchison et al. (2002b) no difference with regard to craving was found between the DRD4 $\mathrm{S}$ and $\mathrm{L}$ groups (only between craving after alcohol consumption versus a placebo in the DRD4 L group). A differential response in subjective craving for the DRD4 $\mathrm{S}$ and $\mathrm{L}$ group has mainly been found in individuals who were being treated with olanzapine (Hutchison et al., 2003; Hutchison et al., 2006).

Previous research by Hutchison et al. (2002b) showed that heavy drinkers with the DRD4 $\mathrm{L}$ allele reported being less aroused and less subjectively high than the DRD4 S participants across three alcoholic drinks. It has been suggested that the DRD4 L individuals might be more sensitive to "self-medicate" in order to enhance their dopamine levels due to their lower (baseline) levels of stimulation. The present study does not support this hypothesis. The DRD4 L participants showed, on average across both exposure trials, significantly higher levels of subjective arousal and lower levels of subjective craving compared with the DRD4 S participants. Both findings are unexpected based on the idea of understimulation playing an important role in the tendency for DRD4 L individuals to engage in drinking (Hutchison ef al., 2002b).

The marginally significant finding that participants with a $C$ allele reported more craving for alcohol during cue exposure to an alcoholic beverage as their weekly alcohol consumption increased could imply that especially the $C$ allele carriers, become increasingly sensitive to the effects of alcohol as they engage in heavier drinking patterns. An increased experience of craving after alcohol exposure might render them more sensitive to engage in further drinking. The lack of a similar finding for craving on the VAS could be due to the single item measurement. The DAQ consists of 14 items, which tap different subtle subcomponents of craving, something which might not be assessed with a single item. The CNRT interaction with saliva suggests that the $C$ allele carriers relatively low on baseline saliva production respond with more physiological cue-reactivity to alcohol cues than their TT counterparts relatively low on baseline saliva.

Although in the present study, overall, no strong differences were found between the genotype groups, with regard to alcohol use or cue-reactivity, the DRD4 VNTR and the CNRT SNP are still important polymorphisms with regard to pharmacological treatment. As the research by Hutchison et al. $(2003,2006)$ showed, the response to the DA antagonist olanzapine was moderated by the DRD4 VNTR, with a better response (less craving and less drinking) in the DRD4 L individuals. 
Since also the endocannabinoid system is involved in addictive behaviours, future research is needed to develop and test medications that interact with the endocannabinoid receptor and reduce the urge to use alcohol or drugs. Animal research and preliminary human studies with the $\mathrm{CB} 1$ receptor antagonist rimonabant are promising (Le Foll and Goldberg, 2005). Possibly, individuals with specific CB1 receptor polymorphisms such as the rs2023239 will benefit more from treatment with $\mathrm{CB} 1$ antagonists such as rimonabant than individuals not carrying this polymorphism. Future research will elucidate these topics. Additionally, more research is necessary to investigate the role of cue-elicited craving as a potential new endophenotype. In the quest for specific genes that are involved in the sensitivity for developing alcohol and drug related problems it is recommended to measure subcomponents of the heterogeneous disease such as alcohol (or drug) dependence. The chance of identifying relevant genes and polymorphisms is optimized this way, which is beneficial for a more targeted treatment and prevention.

\section{ACKNOWLEDGEMENTS}

The authors would like to acknowledge Ellen Lambrichs, Brad Pemberton and Andrew Smolen for the genotyping and Lotte Voorham for help in the data collection. This research was supported in part by a 'VIDI' grant from the Dutch National Science Foundation (N.W.O.) to RWW (452.02.005). 
DRD4 AND CNRI NOT STRONGLY RELATED TO ALCOHOL CUE-REACTIVITY 



\section{CHAPTER 7}

\section{GENERAL DISCUSSION}




\section{OVERVIEW AND DISCUSSION OF EMPIRICAL FINDINGS}

The goal of this thesis was to investigate several cognitive and biological individual differences that might be related to the vulnerability for developing alcohol-related problems. As a general context for discussing the outcomes of the studies in this thesis, first two currently influential neurobiological theories of the development of addiction are briefly described. Second, the concept of endophenotypes is discussed. Then, the results of the different studies are discussed within this general framework (except for the clinical study in Chapter 4, which is discussed in the section on clinical implications). To end, I discuss some clinical implications, methodological issues and suggestions for further research.

The first currently influential neurobiological theory of addiction was already introduced in the general introduction; Robinson and Berridge's incentive sensitization theory (1993). The essence of this theory is that when an addiction develops, the mesolimbic dopamine system becomes sensitized. This results in the attribution of incentive salience to addiction-related stimuli. A second view on addiction is a more developmental view, in which several stages in the process towards addiction can be distinguished with different neurotransmitters and brain structures that play a crucial role in each of these stages (e.g. Everitt \& Robbins, 2005; Kalivas \& Volkov, 2005). In the initial phase, acute psychomotor stimulant effects of the drug, associated with a strong dopamine release in the nucleus accumbens (shell), are important. Dopamine release is required for experiencing a drug high. Gradually, as the drug use becomes more habitual (transition to addiction stage), structural neuroadaptations take place that involve glutamate projections from the prefrontal cortex to the nucleus accumbens (core). According to this view, and contrary Robinson and Berridge, once an individual is addicted (end stage), mesolimbic dopamine release into the nucleus accumbens shell is no longer essential for the experience of craving. Varieties of this second theory have also been called "incentivehabit" theory (Di Chiara, 2000; Mogg et al., 2005). Stimuli signaling drug availability result in a hyperactive prefrontal cortex and excitatory glutamatergic drive to the nucleus accumbens core. This has been hypothesized to be a final common pathway for eliciting craving and compulsive drug seeking. This account can explain the excessive motivational salience attributed to drug-related stimuli when present and the reduced decision making ability and reduced incentive salience of natural rewards during basal conditions.

The second general theoretical issue relevant for the discussion of the findings in this thesis is the concept of endophenotypes. Endophenotypes are intermediate phenotypes that can bridge the gap between the more distal genotype and disease phenotype. According to Gottesman and Gould (2003), several criteria exist that 
can be useful for the identification of endophenotypes: 1) the endophenotype should be associated with the disease, 2 ) it is heritable, 3) it is state-independent (a 'trait'), 4) endophenotype and disease co-segregate within families and 5) in nonaffected family members, the endophenotype is more prevalent than within the general population. Gottesman and Gould differentiate between endophenotypes that have genetic underpinnings, and biological markers, that are not heritable but mark the disorder. Endophenotypes can help to identify candidate genes that are related to the disorder of interest.

Cue-elicited craving has been suggested to be a potentially promising endophenotype candidate (Hutchison et al., 2002a; 2002b). When we look at the data presented in this thesis, we find some support for this. The data from the cue exposure study presented in Chapter 5, show that heavy drinkers carrying a copy of the $G$ allele of the OPRM1 receptor gene polymorphism, report more craving for alcohol after exposure to their favorite alcoholic drink compared with the individuals homozygous for the major A allele. The $G$ allele participants also reported a significantly higher lifetime prevalence of drug use than AA participants. Perhaps this is related to stronger cue-induced craving for other drugs as well (this was not tested). Thus, the endophenotype can discriminate between two groups that differ in a polymorphism of a potentially relevant candidate gene involved in addictive behaviours.

In addition, a tentative interaction with alcohol use was found such that the relatively 'light' (moderately heavy) drinking $G$ allele carriers reported the strongest increase in craving. Possibly, the OPRM 1 gene is more involved in the first association of pleasure, the hedonic effect of alcohol use, which seems especially important in the initial phase of habitual drinking (Everitt \& Robbins, 2005). Other genes might play a role in making a person more liable for a faster sensitization of the brain reward system leading to compulsive drug seeking and taking behavior (Robinson and Berridge, 2003). Peciña and Berridge (2000) showed that activation of opioid neurotransmitter receptors within the medial caudal subregion of the nucleus accumbens shell caused an increase in positive affective reactions to sweet tastes. The nucleus accumbens shell may therefore be a possible location for the "liking" component Robinson and Berridge (1993) refer to. Ray and Hutchison found higher happiness scores for the $G$ allele participants after alcohol administration. Research from Kampov-Polevoy has shown that there is indeed an association between sweet preference and excessive alcohol intake, in animals as well as humans (Kampov-Polevoy et al., 1999; 2001).

The CNRI SNP interacted with alcohol use such that the heavier drinking C allele carriers reported more craving for alcohol (Chapter 6). This interaction with alcohol use was exactly the opposite of the interaction found for the A1 18G poly- 
morphism of the OPRM1 receptor gene (Chapter 5), as described above, where the $G$ allele carriers who were still relatively 'moderate' drinkers reported the largest increase in craving after alcohol exposure. It remains speculative, but the influence of the CNR1 polymorphism might become more pronounced as consumption moves from habitual to more compulsive use. At least this would explain why the heavier drinking $\mathrm{C}$ allele participants tended to crave more during alcohol exposure.

When we look at the cue exposure data in relation to the DRD4 VNTR we do not find strong evidence for a moderating effect of this polymorphism on cueelicited craving after alcohol exposure. Contrary to our hypotheses, we found that, on average over both exposure trials, the DRD $4 \mathrm{~L}$ participants reported less craving for alcohol than the DRD4 S participants.

In the naltrexone treatment study (Chapter 4), FHPs reported higher craving scores than FHNs at pretest (note that this was not cue-elicited craving, but overall craving experienced during the past week). Data from the larger sample of alcohol-dependent patients (Chapter 3) did not reveal any differences between FHNs and FHPs with regard to craving. Together, these findings suggest that cue-induced craving may be a better endophenotype than measures of craving without a cue.

Alcohol-induced heart-rate (HR) increase during the ascending limb of the blood alcohol curve has been suggested to be a psychophysiological index for psychomotor stimulation after alcohol consumption (Conrod et al., 2001). It has been shown to discriminate between FHPs and FHNs. Individuals who are at an increased risk show a stronger alcohol-induced HR increase than individuals at a lower risk (Conrod et al., 1997; Peterson et al., 1996). As such, alcohol-induced HR seems to meet at least some of the proposed criteria of an endophenotype. Implicit cognitions such as alcohol arousal (Wiers et al., 2002) and approach associations (Palfai \& Ostafin, 2003) and attentional bias (Franken, 2003; Robinson \& Berridge, 2001) have all been hypothesized to reflect incentive sensitization of the brain reward center and could possibly be more cognitive endophenotypes. In the alcohol challenge study with heavy drinkers (Chapter 2) it was investigated whether these three implicit cognitive processes were positively correlated with alcohol-induced heart-rate changes during the ascending blood alcohol curve. This hypothesis was not confirmed. Implicit arousal associations even tended to be negatively correlated with alcohol-induced HR change. It is possible that the results would have been different if more FHPs had participated in the study (since they show the strongest alcohol-induced HR increase). Alcohol-induced HR acceleration is perhaps not a reliable measure of psychomotor stimulation in $\mathrm{FHNs}$.

There is evidence that points in the direction that heavy drinking FHPs tend to show stronger implicit arousal associations than FHNs (Wiers, in press b). How- 
ever, the data from the patient sample in Chapter 3 suggest that that FHP alcoholics $(n=22)$ and FHN alcoholics $(n=43)$ do not differ in implicit arousal and approach associations or in attentional bias. If implicit alcohol associations and attentional bias indeed tap incentive sensitization as has been hypothesized, one would expect hat these measures show positive associations with each other, with craving, with positive expectancies of a high dose of alcohol and with measures that give an indication of the drinking problem (e.g. age of onset of heavy drinking, years of problematic drinking, alcohol use and alcohol problems). One would also expect alcohol-dependent patients to display stronger implicit alochol-related cognitions than controls, who do not suffer from a sensitized brain reward center.

Overall, significant findings were rather scarce and inconsistent. Once, a significant correlation was found between the arousal and approach IAT, in the sample of heavy drinkers (Chapter 2), and once between the arousal IAT and the blocked Stroop, in the alcohol-dependent patients (Chapter 3). However, fairly low correlations between implicit measures have been found more often (e.g. Bosson et al., 2000). This could be due to low reliabilities or the different measures could tap into different processes. In the patients, none of the implicit measures correlated significantly with craving or positive explicit expectancies for a high dose of alcohol. When investigating the relationship between implicit measures and alcohol measures, a borderline significant correlation between the arousal IAT and alcohol problems was found in the sample of heavy drinkers. In alcohol-dependent patients a positive correlation was found between the approach-avoidance IAT and alcohol problems. The patients did not differ much in implicit cognitions when compared to controls (Chapter 3). No difference was found on the arousal IAT. The patients only tended to show less strong avoidance associations than the controls.

A remarkable and unexpected finding in the patients was the negative correlation between years of problematic drinking and attentional bias on the blocked Stroop (Chapter 3). Explorative post-hoc analyses revealed that only the 'shortterm' alcoholic patients showed a significant attentional bias both on the blocked and random Stroop, while the Stroop effects for the 'long-term' alcoholics were not significant. For the short-term alcoholics the strongest association between the arousal IAT and the blocked Stroop was found (.40 instead of .26 for whole sample). It was suggested that incentive habit (Di Chiara, 2000; Everitt \& Robbins, 2005; cf. Mogg et al., 2005) processes rather than only incentive sensitization (Robinson \& Berridge, 1993; 2003) could explain these findings.

Taken together, no consistent pattern was found for the implicit measures to be strongly associated with each other, with explicit measures such as craving and positive expectancies or with alcohol measures. Also, no evidence was found for a difference between FHPs and FHNs on implicit cognitions. Neither were there con- 
vincing differences between patients and healthy controls on these measures. Thus, in these correlational studies (Chapter 2 and 3) little support was found for implicit measures to be good endophenotypes of alcohol-related problems. This could be partly due to methodological issues such as the counterbalanced presentation of the several IATs and Stroops, which may have introduced noise in the data. Another explanation is that once addicted, implicit measures are not a good endophenotype to distinguish between FHPs and FHNs. Note that a difference in implicit arousal associations was found between heavy drinking FHPs and FHNs who were not yet addicted (Wiers, in press b). Together, these findings appear to be more in line with incentive habit than with incentive sensitization.

In addition to (cue-elicited) craving, alcohol-induced HR increase and implicit cognitions, a fourth possible endophenotype could be explicit positive alcohol (arousal) expectancies. It has been found that FHP alcoholics report stronger positive alcohol expectancies for a high dose than FHNs (Wiers et al., 2000). Also, children of alcoholics hold more negative alcohol expectancies before initiation of drinking than children of non-alcoholics (due to aversive learning) and show a shift towards more positive expectancies after they start experimenting with alcohol (genes come into play; Wiers et al., 1998). Thus, as explicit alcohol expectancies seem to reflect underlying biological responses to alcohol (next to environmental influences such as social learning), one might expect that they are related to the physiological response after alcohol consumption, such as alcohol-induced HR change (Chapter 2). Arousal expectancies of the immediate effects of alcohol would be expected to be positively associated with HR increase during the ascending BAC, while late sedation expectancies would be expected to show an association with HR decrease during the descending BAC. Although these hypotheses could not be confirmed, it was found that participants with strong immediate arousal expectancies of a high dose reached the peak of their blood alcohol concentration $(B A C)$ the fastest, indicating that these expectancies indeed reflect personal experience. Furthermore, participants with strong explicit immediate arousal expectancies reported higher alcohol consumption (even within the sample of heavy drinkers) and participants who expected to become sedated 1.5 hours after drinking a high dose of alcohol reported more alcohol-related problems. In alcohol-dependent patients (Chapter 3), significant associations were found as well, between expectancies, craving and several alcohol measures (e.g. alcohol use, problems, age of onset). No differences were found between alcohol-dependent FHNs and FHPs on explicit positive and negative expectancies (Chapter 3). However, the patients reported significantly stronger positive and tension reduction expectancies than controls. Taken together, explicit measures remain a valuable tool in the assessment of cognitive factors that might contribute to excessive drinking 
behavior. Implicit or indirect measures could be an additional tool to rather than a replacement of explicit measures.

Subjective arousal as indicator of cue reactivity seemed to reveal a pattern of understimulation in the data of the $G$ allele carriers of the OPRM 1 receptor gene. The $G$ allele participants who reported low levels of baseline arousal showed a larger increase after alcohol exposure compared with the A allele participants who were low on baseline arousal. These data are in line with the findings of Gianoulakis et al. (1996a, 1996b) who found beta-endorphinergic underarousal in FHPs. This 'underarousal' might be compensated by using alcohol or drugs. The DRD4 L participants reported, on average over both exposure trials, more subjective arousal during cue exposure than the DRD4 S participants. The groups did not differ in baseline arousal scores. This would not be expected based on the suggestion done by Hutchison et al. (2002b) that DRD4 L individuals have lower baseline levels of stimulation which can be compensated by alcohol or drug use. The finding that DRD4 L participants did not only report an increase in arousal during the alcohol exposure, but also during the water exposure might be explained by a more general appetitive response in this subgroup. All participants knew they were participating in an alcohol experiment and were therefore probably expecting an alcoholic beverage. The water trial may have been a cue for the alcohol trial, leading to an overall increase in arousal in the DRD $4 \mathrm{~L}$ group.

Saliva production has been used more often as a psychophysiological index of cue reactivity after exposure to appetitive cues such as food (e.g. Nederkoorn \& Jansen, 2002) and alcohol (e.g. Monti et al., 1987). In this thesis, evidence was found as well that in response to the exposure to their favorite alcoholic beverage, the heavier drinking participants showed a stronger salivary reactivity (Chapter 5). The DRD4 VNTR polymorphism tended to moderate salivary reactivity such that DRD4 L participants showed a larger beverage effect (alcohol-water exposure) than the DRD4 S participants (Chapter 6). After power calculations it became evident that with a somewhat larger DRD4 L group, the difference between the DRD4 S and $\mathrm{L}$ groups would have been significant. Thus, it seems that DRD4 $\mathrm{L}$ individuals respond with a stronger salivary response after alcohol exposure. The CNR1 SNP interacted with baseline saliva, such that the $C$ allele participants low on baseline saliva production tended to show the largest saliva reactivity after alcohol exposure compared with the T allele participants low on baseline saliva (Chapter 6). These findings are similar to what was found for the A118G polymorphism (OPRM1 gene) with baseline measures of arousal and craving (VAS). Taken together, it seems as though some form of understimulation (in craving, arousal or saliva) leads to a stronger increase in these subjective and psychophysiological responses 
in individuals carrying a copy of one of the minor alleles ( $G$ or $C$ ) of either the OPRM1 or CB1 receptor gene, respectively.

The main findings of the studies presented in Chapters 2, 3, 5 and 6 were described above. Below the main findings of Chapter 4 will be reported, followed by several clinical implications.

\section{CLINICAL IMPLICATIONS}

In Chapter 4, we were interested whether implicit and explicit measures were able to predict treatment outcome in alcohol-dependent patients receiving 'standard care' in combination with naltrexone treatment. Although the sample ended up being rather small, which precludes generalizations to a larger sample of alcoholic patients in treatment, several potentially interesting differences emerged between patients who relapsed and who remained abstinent. Also between FHPs and FHNs several differences were found.

Naltrexone has been found to be especially effective in FHPs (Monterosso et al., 2001), probably due to naltrexone blocking their sensitive, heritable betaendorphinergic response. In our study, contrary to what was hypothesized, patients who relapsed during the first three months of treatment were predominantly FHPs. Further, FHPs who relapsed tended to be older than the FHPs who remained abstinent. It would be worthwhile investigating whether primarily FHPs in the relatively early phase of addiction would benefit more from naltrexone treatment and older FHP alcoholics with longer alcoholism histories more from treatment with acamprosate. Naltrexone blocks the alcohol-induced high (Volpicelli et al., 1995) and interacts with the opioid system, a system which might be especially important in the early phase of an addiction (Everitt \& Robbins, 2005). Acamprosate modulates activity of the glutamate system, which has been found to be of importance in the end-stage of addiction (e.g. Everitt \& Robbins, 2005; Kalivas \& Volkov, 2005). Also, combining naltrexone and acamprosate seems promising (e.g. Feeney et al., 2006; Kiefer et al., 2003).

The relapsers further tended to show stronger implicit alcohol avoidance associations on the IAT and were less distracted by alcohol-related words on the Stroop (even faster at responding to the alcohol words compared with neutral words) compared with the patients who remained abstinent. When the data was, more exploratively, investigated over the course of the first 6 weeks of treatment, this pattern seemed to remain stable. Thus, it seems as though these implicit cognitions did not change over treatment. It would be interesting to see whether these findings could be replicated in a larger sample, and if yes, if these associations would be related to anxiety of relapsing or anxiety of being exposed to alcohol cues. Perhaps 
cue exposure could be particularly helpful for these 'avoidant' individuals in practicing high-risk situations?

Craving at treatment admission did not predict relapse. It would be expected that patients with high levels of craving would benefit most from naltrexone (Monterosso et al., 2001). Over the course of treatment the data seemed to suggest that FHPs who relapsed reported constant levels of craving, while the FHPs who remained abstinent reported a decrease in craving. Lack of medication compliance in the subsample of FHPs who relapsed may have influenced treatment outcome, emphasizing the importance of medication compliance.

Finally, when comparing FHPs with FHNs, it became evident that these two groups differed at treatment admission. The FHPs were less days abstinent, reported more craving, less desire to quit drinking on the Alcohol Intentions Questionnaire $(A I Q)$ and a lower success expectation of achieving their drinking goal than the FHNs. Over the course of treatment, it seemed that FHPs remained, on average, less motivated to quit drinking than $\mathrm{FHNs}$ and they even tended to show a decrease in motivation over time. FHPs also seemed to have lower success expectations than $\mathrm{FHNs}$ over the course of treatment. It could be valuable to administer the $\mathrm{AIQ}$ at treatment admission as a measure of motivation to change drinking behavior. Especially FHPs might benefit from additional motivational interviewing techniques to increase their motivation to change their drinking behavior and their self-efficacy to do this.

\section{METHODOLOGICAL ISSUES AND SUGGESTIONS FOR FUTURE RESEARCH}

Limitations to the several studies were mentioned in the separate chapters. However, some issues remain to be mentioned. Part of the studies was correlational (Chapter 2, 3). This makes it difficult to draw conclusions with regard to whether or not the measures used in these studies might be called endophenotypes. An endophenotype implies some form of causal relationship with the disorder of interest, which cannot be tested in a correlational design. Prospective studies are warranted in which for example implicit measures are used to predict future drinking behavior (e.g. Thush et al., in press). To investigate potentially promising endophenotypes and their influence on future drinking behavior, it is recommended to compare high versus low risk groups in which the disorder has not manifested itself (e.g. children of alcoholics versus children of non-alcoholics). In comparing alcoholdependent patients versus healthy controls, differences can be a cause as well as a consequence of the alcohol problem. A similar problem arises when FHPs are 
compared with FHNs; if a difference between the groups is found, this could be due to a third (unknown) variable.

Future studies using indirect measures should not assess too many tasks in a row (e.g. practice effects reduce IAT effects) and should optimize the measures that are used. In the IAT for example, it is still not entirely clear whether the contrast category 'neutral' in the unipolar IAT is a good contrast category. Bipolar IATs usually yield higher reliabilities (Houben, 2007). It is worthwile to further investigate whether the lack of a target or attribute contrast category, as in the single-target IAT (e.g. only the target 'Alcohol' combined with bipolar attributes such as 'Arousal' vs 'Sedation'; Karpinski \& Steinman, 2006; Wigboldus et al., 2006) or single attribute IAT (e.g. targets 'Alcohol' an 'Soda' verus one attribute category such as 'Arousal'; Penke et al., in press), is advantageous because it circumvents the problem of relativity of the IAT. In a unipolar or bipolar IAT, associations between alcohol and arousal can also be due to associations between soda and neutral (unipolar) or soda and sedation (bipolar). A single target or single attribute IAT assesses only one association instead of two. A limitation of the IAT is that it measures associations. It says nothing about directionality. For example in the arousal IAT, it is unclear whether a faster response in the combination phase where alcohol and arousal words share the same response key has to do with the association 'When I drink alcohol, I become energetic' or 'When I'm energetic, I would like to drink alcohol'. Future research could focus on implicit measures that assess the time component as well (see Wiers, in press a).

It has been hypothesized that arousal associations (Wiers et al., 2002), approach associations (Palfai \& Ostafin, 2003) and attentional bias for or distraction by alcohol-related stimuli (Franken, 2003; Robinson \& Berridge, 2003) reflect the underlying, biological process of incentive sensitization. As such, these three implicit measures could be regarded as potential endophenotypes. However, in some of the studies presented here, only correlations were tested between the different implicit measures and between implicit measures on the one hand and explicit measures (craving, expectancies), alcohol measures (e.g. use, problems, and age of onset) and physiological measures (alcohol-induced heart-rate acceleration) on the other hand. Finding a positive association between the implicit measures (which only occurred twice) does not imply that these measures indeed tap the hypothesized process of incentive sensitization. A correlation only suggests that the tests share some common variance, but it remains unclear what that variance exactly is and by what it is caused. By simply correlating these measures we cannot test the theory that the implicit measures indeed tap incentive sensitization. Neither do we falsify the theory when an association between the measures is not found. Lack of strong and consistent relations between the implicit measures and drinking meas- 
ures (e.g. use, problems, and age of onset) seems to indicate, however, that at least in the present studies, the measures do not relate to drinking behavior. This could be due to restriction of range because the samples consisted of either heavy drinkers (Chapter 2) or alcohol-dependent patients (Chapter 3). A broader range in drinking behavior could have resulted in more variance. A similar explanation could hold for the lack of a positive association between the implicit measures and alcohol-induced heart-rate acceleration (Chapter 2). Since the sample did not contain many FHPs (who were expected to show the strongest alcohol-induced HR increase), a lack of enough variation in this measure might have resulted in a lack of strong correlations between HR and implicit measures. It is also possible that alcohol-induced HR increase reflects incentive sensitization only in FHPs and not in FHNs.

Future research that aims at investigating whether the indirect measures indeed tap into the process of incentive sensitization, as hypothesized, should focus more on a direct measurement of incentive sensitization or "wanting". Functional magnetic resonance imaging ( $\mathrm{fMRI}$ ) offers the possibility to this. There is evidence that while viewing alcohol pictures, increased activation in areas such as the nucleus accumbens is associated with subjective craving in alcohol-dependent patients, but not in social drinkers (e.g Myrick et al., 2004). Also, a lower availability of D2-like receptors in the ventral striatum of alcoholics has been associated with severity of alcohol craving and with a greater cue-induced activation of the medial prefrontal cortex and anterior cingulate (Heinz et al., 2004). A dopaminergic dysfunction in the ventral striatum may attribute incentive salience to alcohol-related stimuli, so that alcohol cues elicit craving and excessive activation of brain areas associated with attention and behavior control. These fMRI findings suggest that a subjective experience such as craving can be directly 'reduced' to brain activation in specific areas in the reward circuitry. Gottesman and Gould (2003) suggest that fMRI analysis of participants performing certain tasks in the scanner (e.g. cue exposure), may be a more sensitive endophenotype than neuropsychological measurement. If arousal and approach associations and attentional bias indeed tap the process of incentive sensitization, one might expect that activation in brain reward areas involved in incentive sensitization, during exposure to alcohol-related pictures is positively correlated with these implicit measures. It would also be interesting to assess these measures while the participant is receiving naltrexone. If naltrexone can change the strength of implicit alcohol associations (which does not seem to be the case when we look at the data over the course of naltrexone treatment presented in Chapter 4), this should result in less brain activation in the reward center which should correlate with smaller IAT and Stroop effects. 
Thus far, no consistent relation has been found between a FHP and presence of the $G$ allele of the OPRMI receptor gene. It is recommended that future clinical trials with naltrexone assess and report $\mathrm{FH}$ status and craving and have all participants genotyped for the A1 18G polymorphism. Genotyping can promote optimal patient-treatment matching. In addition, it would be interesting to further investigate whether naltrexone is more effective in younger FHPs or $G$ allele carriers. Further, in cooperation with Kampov-Polevoy it will be investigated whether the $G$ allele is associated with sweet preference. A sub sample of the heavy drinking participants who took part in the cue-reactivity study in relation to the A1 18G polymorphism (Chapter 5) agreed on filling out the Sweet Preference Questionnaire developed by Kampov-Polevoy. These data remain to be analyzed. Preliminary analyses relating the three receptor gene polymorphisms discussed in this thesis (OPRM1, DRD4, CNR1) to several implicit measures (e.g. memory associations on the IAT) suggest that associations between these polymorphisms and implicit measures exist.

Research regarding the identification of potential risk genes and their relation to pharmacotherapeutic treatment looks promising. In animals, the CB1 receptor antagonist rimonabant (SR141716) has shown to block the release of dopamine induced by several drugs as well as the rewarding effects of the active ingredient of cannabis ( $\Delta^{9}-\mathrm{THC}$; see Carai et al., 2005; Le Foll \& Goldberg, 2005, for reviews). Also, a reduction in alcohol consumption has been demonstrated. An ongoing clinical trial by the National Institute on Alcohol Abuse and Alcoholism (NIAAA) is investigating whether rimonabant will decrease alcohol consumption in heavy drinkers. It would be interesting to further investigate whether it is an effective medication for alcohol-dependent patients and if patients carrying a $\mathrm{C}$ allele of the CNR1 SNP (rs2023239) would benefit most. If this would indeed be the case, this finding could possibly extend to other drug-related disorders and eating disorders (e.g. obesity) as well (rimonabant has also been found to be effective in reducing food intake and body weight, Carai et al., 2005).

\section{IN CLOSING}

No evidence was found for implicit cognitions such as arousal and approach associations and attentional bias to be positively associated with alcohol-induced heartrate increase during the rising limb of the blood alcohol curve. Also, in general, no strong and consistent associations were found between the three implicit measures or between the implicit measures and alcohol measures such as use, problems and age of onset. In addition, few differences were found between FHP and FHN alcoholics or between alcoholic patients and healthy controls. Explicit measures such as 
alcohol expectancies and craving, rather than implicit measures, were related to alcohol measures, and were able to distinguish patients from controls. Possibly, implicit measures are better endophenotypes in the initial phase of a developing addiction. This would be more in line with incentive habit theories.

Genes are of great influence in predisposing individuals to engage in alcohol and drug abuse. Genetic variations or polymorphisms can moderate the response to alcohol and drugs, for example by altering receptor function, receptor expression and drug metabolism. Medication that targets specific receptors that are affected by these genetic polymorphisms might moderate treatment effectiveness. Given the finding that heavy drinking $G$ allele carriers experienced stronger craving after alcohol exposure than $A A$ individuals, naltrexone might possibly be especially effective in patients carrying a $G$ allele of the OPRM 1 receptor gene by decreasing craving. A copy of the $G$ allele of the $A 118 G$ polymorphism might render an individual sensitive to experiment more often with drugs in general. This could be inferred from the higher drug use scores that were found in this particular group. Naturally, genes always interact with other genes and environmental factors, thus presence of this polymorphism does not lead to the phenotype of alcohol or drug dependence through one final common pathway. Although no strong evidence was found for a moderating effect of the DRD4 VNTR and the CNRI SNP on cue reactivity, both polymorphisms remain interesting candidates for pharmacotherapeutic treatment. The identification of genes that are involved in substance use disorders will enhance our understanding of addictive behaviors, such that new medications can be developed and prevention and treatment can be targeted more effectively. 


\section{REFERENCES}

American Psychiatric Association (1994). Diagnostic and statistical manual of mental disorders (DSM-IV). Washington D.C.: American Psychiatric Association.

Anchordoquy, H. C., McGeary, C., Liu, L., Krauter, K. S., \& Smolen, A. (2003). Genotyping of three candidate genes following whole genome preamplification of DNA collected from buccal cells. Behavioural Genetics, 33, 73-78.

Anton, R. F. (2000). Obsessive-compulsive aspects of craving: development of the Obsessive Compulsive Drinking Scale. Addiction, 95, S211-217.

Arias, A., Feinn, R., \& Kranzler, H. R. (2006). Association of an Asn40Asp (A1 18G) polymorphism in the mu-opioid receptor gene with substance dependence: A meta-analysis. Drug and Alcohol Dependence, 83, 262-268.

Asendorpf, J. B., Banse, R., \& Mücke, D. (2002). Double dissociation between implicit and explicit personality self-concept: The case of shy behavior. Journal of Personality and Social Psychology, 83, 380393.

Asghari, V., Sanyal, S., Buchwaldt, S., Paterson, A., Jovanovic, V., \& Van Tol, H. H. (1995). Modulation of intracellular cyclic AMP levels by different human dopamine D4 receptor variants. Journal of Neurochemistry, 65, 1157-1165.

Bart, G., Kreek, M. J., Ott, J., LaForge, K. S., Proudnikov, D., Pollak, L., \& Heilig, M. (2005). Increased attributable risk related to a functional mu-opioid receptor gene polymorphism in association with alcohol dependence in central Sweden. Neuropsychopharmacology, 30, 417-422.

Basavarajappa, B. S., Cooper, T. B., \& Hungund, B. L. (1998). Chronic ethanol administration downregulates cannabinoid receptors in mouse brain synaptic plasma membrane. Brain Research, 793, 212-218.

Basavarajappa, B. S., \& Hungund, B. L. (2002). Neuromodulatory role of the endocannabinoid signaling system in alcoholism: an overview. Prostaglandins, Leukotrienes and Essential Fatty Acids, 66, $287-$ 299.

Bechara, A., Noel, X., \& Crone, E. A. (2006). Loss of Willpower: Abnormal Neural Mechanisms of Impulse Control and Decision Making in Addiction. In R. W. Wiers \& A. W. Stacy (Eds.), Handbook of implicit cognition and addiction (pp. 215-232). Thousand Oaks, CA, US: Sage Publications.

Bergen, A. W., Kokoszka, J., Peterson, R., Long, J. C., Virkkunen, M., Linnoila, M., \& Goldman, D. (1997) $\mathrm{Mu}$ opioid receptor gene variants: lack of association with alcohol dependence. Molecular Psychiatry, 2, 490-494.

Beringer, J. (1996). Experimental Run Time System (ERTS), Version 3. 18. Frankfurt, Germany: BeriSoft.

Berridge, K. C. (2001). Reward learning: Reinforcement, incentives, and expectations, in The psychology of learning and motivation: Advances in research and theory (Medin D ed), pp 223 278. Academic Press, San Diego.

Berridge, K. C., \& Robinson, T. E. (1998). What is the role of dopamine in reward: Hedonic impact, reward learning, or incentive salience? Brain Research Reviews, 28, 309-369.

Bijl, R. V., Ravelli, A., \& van Zessen, G. (1998). Prevalence of psychiatric disorder in the general population: Results of the Netherlands Mental Health Survey and Incidence Study (NEMESIS). Social Psychiatry and Psychiatric Epidemiology, 33, 587-595.

Blanchard, K. A. (1999). A moderational model of alcohol treatment outcome: The relationship between motivation and cognitive deficits. (substance abuse). Dissertation Abstracts International: Section B: The Sciences and Engineering, 60, 2934.

Bond, C., LaForge, K. S., Tian, M., Melia, D., Zhang, S., Borg, L., Gong, J., Schluger, J., Strong, J. A., Leal, S. M., Tischfield, J. A., Kreek, M. J., \& Yu, L. (1998). Single-nucleotide polymorphism in the hu- 
man mu opioid receptor gene alters beta-endorphin binding and activity: possible implications for opiate addiction. Proceedings of the National Academy of Sciences of the United States of America, 95, 9608-9613.

Bosson, J. K., Swann, W. B., \& Pennebaker, J. W. (2000). Stalking the perfect measure of implicit selfesteem: The blind men and the elephant revisited? Journal of Personality and Social Psychology, 79, $631-643$.

Bouza, C., Angeles, M., Munoz, A., \& Amate, J. M. (2004). Efficacy and safety of naltrexone and acamprosate in the treatment of alcohol dependence: a systematic review. Addiction, 99, 81 1-828.

Bruce, G., \& Jones, B. T. (2006). Methods, measures and findings of attentional bias in substance use, abuse and dependence, in Handbook of Implicit Cognition and Addiction (Wiers R. W., Stacy A. W. eds), pp 135-149. SAGE, Thousand Oaks, CA.

Brunelle, C., Assaad, J. M., Barrett, S. P., Avila, C., Conrod, P. J., Tremblay, R. E., \& Pihl, R. O. (2004). Heightened Heart Rate Response to Alcohol Intoxication Is Associated With a Reward-Seeking Personality Profile. Alcoholism: Clinical and Experimental Research, 28, 394-401.

Carai, M. A., Colombo, G., \& Gessa, G. L. (2005). Rimonabant: the first therapeutically relevant cannabinoid antagonist. Life Sciences, 77, 2339-2350.

Carver, C. S., \& White, T. L. (1994). Behavioral inhibition, behavioral activation, and affective responses to impending reward and punishment: The BIS/BAS Scales. Journal of Personality and Social Psychology, 67, 319-333.

Cloninger, C. R. (1987). Neurogenetic adaptive mechanisms in alcoholism. Science, 236, 410-416.

Cohen, J. (1992). A power primer. Psychological Bulletin, 112, 155-159.

Comings, D. E., Muhleman, D., Gade, R., Johnson, P., Verde, R., Saucier, G., \& MacMurray, J. (1997). Cannabinoid receptor gene (CNR1): association with i.v. drug use. Molecular Psychiatry, 2, 161-168.

Conrod, P. J., Peterson, J. B., \& Pihl, R. O. (2001). Reliability and validity of alcohol-induced heart rate increase as a measure of sensitivity to the stimulant properties of alcohol. Psychopharmacology, 157, 20-30.

Conrod, P. J., Peterson, J. B., Pihl, R. O., \& Mankowski, S. (1997). Biphasic effects of alcohol on heart rate are influenced by alcoholic family history and rate of alcohol ingestion. Alcoholism: Clinical and Experimental Research, 21, 140-149.

Cox, W. M., Brown, M. A., \& Rowlands, L. J. (2003). The effects of alcohol cue exposure on nondependent drinkers' attentional bias for alcohol-related stimuli. Alcohol and Alcoholism, 38, 45-49.

Cox, W. M., Fadardi, J. S., \& Pothos, E. M. (2006). The Addiction-Stroop Test: Theoretical Considerations and Procedural Recommendations. Psychological Bulletin, 132, 443-476.

Cox, W. M., Hogan, L. M., Kristian, M. R., \& Race, J. H. (2002). Alcohol attentional bias as a predictor of alcohol abusers' treatment outcome. Drug and Alcohol Dependence, 68, 237-243.

Cox, W. M., Yeates, G. N., \& Regan, C. N. (1999). Effects of alcohol cues on cognitive processing in heavy and light drinkers. Drug and Alcohol Dependence, 55, 58-58.

Cunningham, W. A., Preacher, K. J., \& Banaji, M. R. (2001). Implicit attitude measures: Consistency, stability, and convergent validity. Psychological Science, 12, 163-170.

Dai, X., Thavundayil, J., \& Gianoulakis, C. (2002). Differences in the responses of the pituitary betaendorphin and cardiovascular system to ethanol and stress as a function of family history. Alcoholism: Clinical and Experimental Research, 26, 1171-1180.

De Bruijn, C., van den Brink, W., de Graaf, R., \& Vollebergh, W. A. M. (2006). The three year course of alcohol use disorders in the general population: DSM-IV, ICD-10 and the Craving Withdrawal Model. Addiction, 101, 385-392.

De Houwer, J. (2006). What Are Implicit Measures and Why Are We Using Them? In R. W. Wiers \& A. W. Stacy (Eds.), Handbook of implicit cognition and addiction (pp. 11-28). Thousand Oaks, CA, US: Sage Publications. 


\section{REFERENCES}

De Houwer, J., Crombez, G., Koster, E. H. W., \& De Beul, N. (2004). Implicit alcohol-related cognitions in a clinical sample of heavy drinkers. Journal of Behavior Therapy and Experimental Psychiatry, 35, 275 286.

Deas, D., May, K., Randall, C., Johnson, N., \& Anton, R. (2005). Naltrexone treatment of adolescent alcoholics: An open-label pilot study. Journal of Child and Adolescent Psychopharmacology, 15, 723728.

Deutsch, R., \& Strack, F. (2006). Reflective and Impulsive Determinants of Addictive Behavior. In R. W. Wiers \& A. W. Stacy (Eds.), Handbook of implicit cognition and addiction (pp. 45-57). Thousand Oaks, CA, US: Sage Publications.

Di Chiara, G. (2000). Role of dopamine in the behavioural actions of nicotine related to addiction. European Journal of Pharmacology, 393, 295-314.

Dick, D. M., \& Foroud, T. (2003). Candidate genes for alcohol dependence: a review of genetic evidence from human studies. Alcoholism: Clinical and Experimental Research, 27, 868-879.

Dunn, M. E., \& Goldman, M. S. (1996). Emperical modeling of an alcohol expectancy memory network in elementary school children as a function of grade. Experimental and Clinical Psychopharmacology, 4, 209-217.

Erblich, J., \& Earleywine, M. (2003). Behavioral undercontrol and subjective stimulant and sedative effects of alcohol intoxication: Independent predictors of drinking habits? Alcoholism: Clinical and Experimental Research, 27, 44-50.

Erblich, J., Earleywine, M., Erblich, B., \& Bovbjerg, D. H. (2003). Biphasic stimulant and sedative effects of ethanol: Are children of alcoholics really different? Addictive Behaviors, 28, $1129-1139$.

Everitt, B. J., \& Robbins, T. W. (2005). Neural systems of reinforcement for drug addiction: from actions to habits to compulsion. Nature Neuroscience, 8, 1481-1489.

Faraone, S. V., Doyle, A. E., Mick, E., \& Biederman, J. (2001). Meta-analysis of the association between the 7-repeat allele of the dopamine $D(4)$ receptor gene and attention deficit hyperactivity disorder. The American Journal of Psychiatry, 158, 1052-1057.

Farmer, K. C. (1999). Methods for measuring and monitoring medication regimen adherence in clinical trials and clinical practice. Clinical Therapeutics., 21, 1074-90.

Fazio, R. H., \& Olson, M. A. (2003). Implicit measures in social cognition research: Their meaning and uses. Annual Review of Psychology, 54, 297-327.

Feeney, G. F. X., Connor, J. P., Young, R. M., Tucker, J., \& McPherson, A. (2006). Combined acamprosate and naltrexone, with cognitive behavioural therapy is superior to either medication alone for alcohol abstinence: A single centres' experience with pharmacotherapy. Alcohol and Alcoholism, 41, 321-327.

Field, M., Mogg, K., \& Bradley, B. P. (2004). Cognitive bias and drug craving in recreational cannabis users. Drug and Alcohol Dependence, 74, 105-111.

Field, M., Mogg, K., \& Bradley, B. P. (2006). Attention to drug-related cues in drug abuse and addiction: component processes. In: Wiers R. W., Stacy A.W., eds. Handbook of Implicit Cognition and Addiction. Thousand Oaks, CA: SAGE, pp. 151-163.

Finn, P. R., \& Justus, A. (1997). Physiological responses in sons of alcoholics. Alcohol Health and Research World, 21, 227-231.

Fleming, M. F., Barry, K. L., \& MacDonald, R. (1991). The Alcohol Use Disorders Identification Test (AUDIT) in a college sample. The International Journal of the Addictions, 26, $1173-1185$.

Franken, I. H. A. (2002). Behavioral approach system (BAS) sensitivity predicts alcohol craving. Journal of Personality and Individual Differences, 32, 349-355.

Franken, I. H. A. (2003). Drug craving and addiction: Intergrating psychological and neuropsychopharmacological approaches. Progress in Neuro-Psychopharmacology \& Biological Psychiatry, 27, 563-579. 
Franken, I. H. A., Rosso, M., \& van Honk, J. (2003). Selective memory for alcohol cues in alcoholics and its relation to craving. Cognitive Therapy and Research, 27, 481-488.

Froehlich, J. C., Zink, R. W., Li, T. K., \& Christian, J. C. (2000). Analysis of heritability of hormonal responses to alcohol in twins: Beta-endorphin as a potential biomarker of genetic risk for alcoholism. Alcoholism: Clinical and Experimental Research, 24, 265-277.

Fromme, K., de Wit, H., Hutchison, K. E., Ray, L., Corbin, W. R., Cook, T. A. R., Wall, T. L., \& Goldman, D. (2004). Biological and behavioral markers of alcohol sensitivity. Alcoholism: Clinical and Experimental Research, 28, 247-256.

Gallate, J. E., Saharov, T., Mallet, P. E., \& McGregor, I. S. (1999). Increased motivation for beer in rats following administration of a cannabinoid CB1 receptor agonist. European Journal of Pharmacology, 370, 233-240.

Gianoulakis, C., de-Waele, J.-P., \& Thavundayil, J. (1996a). Implication of the endogenous opioid system in excessive ethanol consumption. Alcohol, 13, 19-23.

Gianoulakis, C., Krishnan, B., \& Thavundayil, J. (1996b). Enhanced sensitivity of pituitary beta-endorphin to ethanol in subjects at high risk of alcoholism. Archives of General Psychiatry, 53, 250-257.

Goldman, D., \& Bergen, A. (1998). General and specific inheritance of substance abuse and alcoholism. Archives of General Psychiatry, 55, 964-965.

Goldman, M. S., \& Darkes, J. (2004). Alcohol expectancy multiaxial assessment: A memory network-based approach. Psychological Assessment, 16, 4-15.

Goldman, M. S., Darkes, J., \& Del Boca, F. K. (1999a). Expectancy mediation of biopsychosocial risk for alcohol use and alcoholism, in How expectancies shape experience (Kirsch I ed), pp 233-262. American Psychological Association, Washington, DC.

Goldman, M. S., Del Boca, F. K., \& Darkes, J. (1999b). Alcohol expectancy theory: The application of cognitive neuroscience. In H. T. Blane \& K. E. Leonard (Eds.), Psychological theories of drinking and alcoholism (2nd ed., pp. 203-246). New York: Guilford Press.

Goldman, D., Oroszi, G., \& Ducci, F. (2005). The genetics of addictions: uncovering the genes. Nature Reviews. Genetics, 6, 521-532.

Gottesman, I. I., \& Gould, T. D. (2003). The endophenotype concept in psychiatry: Etymology and strategic intentions. American Journal of Psychiatry, 160, 636-645.

Grant, B. F., Dawson, D. A., Stinson, F. S., Chou, S. P., Dufour, M. C., \& Pickering, R. P. (2004). The $12-$ month prevalence and trends in DSM-IV alcohol abuse and dependence: United States, 1991-1992 and 2001-2002. Drug and Alcohol Dependence, 74, 223-234.

Greenwald, A. G., \& Banaji, M. R. (1995). Implicit social cognition: Attitudes, self-esteem, and stereotypes. Psychological Review, 102, 4-27.

Greenwald, A. G., McGhee, D. E., \& Schwartz, J. L. K. (1998). Measuring individual differences in implicit cognition: The implicit association test. Journal of Personality and Social Psychology, 74, 1464-1480.

Greenwald, A. G., Nosek, B. A., \& Banaii, M. R. (2003). Understanding and using the implicit association test: I. An improved scoring algrithm. Journal of Personality and Social Psychology, 85, 197-216.

Harvey, A. G., Watkins, E., Mansell, W., \& Shafran, R. (2004). Cognitive Behavioural Processes across Psychological Disorders: A transdiagnostic approach to research and treatment. New York: Oxford University Press Inc.

Haughey, H. M., Finan, P., Villanueva, R., Ray, L. A., \& Hutchison, K. E. (2005). The cannabinoid receptor gene (CNRT) is associated with the acute subjective effects of alcohol in a sample of heavy drinkers. Alcoholism: Clinical and Experimental Research, 29-Suppl, 132A (abstract).

Heinz, A., Siessmeier, T., Wrase, J., Hermann, D., Klein, S., Grusser, S. M., Flor, H., Braus, D. F., Buchholz, H. G., Grunder, G., Schreckenberger, M., Smolka, M. N., Rosch, F., Mann, K., \& Bartenstein, P. (2004). Correlation between dopamine $D(2)$ receptors in the ventral striatum and central processing of alcohol cues and craving. Americam Journal on Psychiatry, 161, 1783-1789. 


\section{REFERENCES}

Herman, A. I., Kranzler, H. R., Cubells, J. F., Gelernter, J., Covault, J. (2006). Association study of the CNR 1 gene exon 3 alternative promoter region polymorphisms and substance dependence. American Journal of Medical Genetics. Part B Neuropsychiatric Genetics, 141, 499-503.

Herz, A. (1997). Endogenous opioid systems and alcohol addiction. Psychopharmacology, 129, 99-111.

Hoehe, M. R., Kopke, K., Wendel, B., Rohde, K., Flachmeier, C., Kidd, K. K., Berrettini, W. H., \& Church, G. M. (2000). Sequence variability and candidate gene analysis in complex disease: association of mu opioid receptor gene variation with substance dependence. Human Molecular Genetics, 9, 2895 2908.

Hofmann, W., Gawronski, B., Gschwendner, T., Le, H., \& Schmitt, M. (2005). A Meta-Analysis on the Correlation Between the Implicit Association Test and Explicit Self-Report Measures. Personality and Social Psychology Bulletin, 31, 1369-1385.

Hosmer, D, \& Lemeshow, S (1989). Applied logistic regression. New York: John Wiley \& Sons Ltd, pp. $34-$ 36.

Houben, K. (2007). Decoding the Alcohol-IAT: The Implicit Association Test as a Measure of Individual Differences in Implicit Preferences for Alcohol. Unpublished doctoral dissertation. Maastricht University, The Netherlands.

Houben K., \& Wiers R. W. (2006). Assessing Implicit Alcohol Associations with the Implicit Association Test: Fact or Artifact? Addictive Behaviors, 31, 1346-1362.

Huberty, C. J., \& Morris, J. D. (1989). Multivariate analysis versus multiple univariate analyses. Psychological Bulletin, 105, 302-308.

Huijding, J., de Jong, P. J., Wiers, R. W., \& Verkooijen, K. (2005). Implicit and explicit attitudes toward smoking in a smoking and a nonsmoking setting. Addictive Behaviors, 30, 949-961.

Hungund, B. L., \& Basavarajappa, B. S. (2004). Role of endocannabinoids and cannabinoid CB1 receptors in alcohol-related behaviors. Annals of the New York Academy of Sciences, 1025, 515-27.

Hungund, B. L., Szakall, I., Adam, A., Basavarajappa, B. S., \& Vadasz, C. (2003). Cannabinoid CB1 receptor knockout mice exhibit markedly reduced voluntary alcohol consumption and lack alcoholinduced dopamine release in the nucleus accumbens. Journal of Neurochemistry, 84, 698-704.

Hutchison, K. E., LaChance, H., Niaura, R., Bryan, A., \& Smolen, A. (2002a). The DRD4 VNTR polymorphism influences reactivity to smoking cues. Journal of Abnormal Psychology, 11 1, 134-143.

Hutchison, K. E., McGeary, J., Smolen, A., Bryan, A., \& Swift, R. M. (2002b). The DRD4 VNTR polymorphism moderates craving after alcohol consumption. Health Psychology, 21, 139-146.

Hutchison, K. E., Ray, L., Sandman, E., Rutter, M. C., Peters, A., Davidson, D., \& Swift, R. (2006). The effect of olanzapine on craving and alcohol consumption. Neuropsychopharmacology, 31, 13101317.

Hutchison, K. E., Rutter, M. C., Niaura, R., Swift, R. M., Pickworth, W. B., \& Sobik, L. (2004). Olanzapine attenuates cue-elicited craving for tobacco. Psychopharmacology (Berl), 175, 407-413.

Hutchison, K. E., Swift, R., Rohsenow, D. J., Monti, P. M., Davidson, D., \& Almeida, A. (2001). Olanzapine reduces urge to drink after drinking cues and a priming dose of alcohol. Psychopharmacology (Berl), 155, 27-34.

Hutchison, K. E., Wooden, A., Swift, R. M., Smolen, A., McGeary, J., Adler, L., \& Paris, L. (2003). Olanzapine reduces craving for alcohol: a DRD4 VNTR polymorphism by pharmacotherapy interaction. Neuropsychopharmacology, 28, 1882-1888.

Ingialdsson, J. T., Laberg, J. C., \& Thayer, J. F. (2003). Reduced heart rate variability in chronic alcohol abuse: Relationship with negative mood, chronic thought suppression, and compulsive drinking. Biological Psychiatry, 54, 1427-1436.

Jajodia, A., \& Earleywine, M. (2003). Measuring alcohol expectancies with the implicit association test. Psychology of Addictive Behaviors, 17, 126-133. 
Johnsen, B. H., Laberg, J. C., Cox, W. M., Vaksdal, A., \& Hugdahl, K. (1994). Alcoholic subjects' attentional bias in the processing of alcohol-related words. Psychology of Addictive Behaviors, 8, $111-115$.

Jones, B. T., Corbin, W., \& Fromme, K. (2001). A review of expectancy theory and alcohol consumption. Addiction, 96, 57-72.

Jones, B. T., \& Schulze, D. (2000). Alcohol-related words of positive affect are more accessible in social drinkers' memory than are other words when sip-primed by alcohol. Addiction Research, 8, 221-232.

Kalivas, P. W., \& Volkow, N. D. (2005). The Neural Basis of Addiction: A Pathology of Motivation and Choice. American Journal of Psychiatry, 162, 1403-1413.

Kampov Polevoy, A. B., Garbutt, J. C., \& Janowsky, D. S. (1999). Association between preference for sweets and excessive alcohol intake: a review of animal and human studies. Alcohol and Alcoholism, 34, 386-395.

Kampov Polevoy, A. B., Garbutt, J. C., \& Khalitov, E. (2003). Family history of alcoholism and response to sweets. Alcoholism: Clinical and Experimental Research, 27, 1743-1749.

Kampov Polevoy, A. B., Laaksonen, E., Sinclair, D., Heinala, P., \& Alho, H. (2004). Not liking strong sweet solutions marks poor response to naltrexone treatment. Alcoholism: Clinical and Experimenal Research, 28-Suppl, $114 \mathrm{~A}$ (abstract).

Kampov Polevoy, A. B., Tsoi, M. V., Zvartau, E. E., Neznanov, N. G., \& Khalitov, E. (2001). Sweet liking and family history of alcoholism in hospitalized alcoholic and non-alcoholic patients. Alcohol and Alcoholism, 36, 165-170.

Karpinski, A., \& Steinman, R. B. (2006). The Single-Category Implicit Association Test as a measure of implicit social cognition. Journal of Personality and Social Psychology, 91, 16-32.

Kelly, A. B., Masterman, P. W., \& Marlatt, G. A. (2005). Alcohol-related associative strength and drinking behaviours: Concurrent and prospective relationships. Drug and Alcohol Review, 24, 489-498.

Kiefer, F., Jahn, H., Tarnaske, T., Helwig, H., Briken, P., Holzbach, R., Kaempf, P., Stracke, R., Baehr, M., Naber, D., \& Wiedemann, K. (2003). Comparing and combining naltrexone and acamprosate in relapse prevention of alcoholism: A double-blind, placebo-controlled study. Archives of General Psychiatry, 60, 92-99.

Klinger, E., \& Cox, W. M. (2004). Motivation and the Theory of Current Concerns. In W. M. Cox \& E. Klinger (Eds.), Handbook of motivational counseling: Concepts, approaches, and assessment (pp. 327). New York, NY, US: John Wiley \& Sons Ltd.

Kluger, A. N., Siegfried, Z., \& Ebstein, R. P. (2002). A meta-analysis of the association between DRD4 polymorphism and novelty seeking. Molecular Psychiatry, 7, 712-717.

Kokkevi, A., \& Hartgers, C. (1995). EuropASI: European adaptation of a multidimensional assessment instrument for drug and alcohol dependence. European Addiction Research, 1, 208-210.

Kozlowski, L. T., Pillitteri, J. L., Sweeney, C. T., Whitfield, K. E., \& Graham, J. W. (1996). Asking questions about urges or cravings for cigarettes. Psychology of Addictive Behaviors, 10, 248-260.

Kramer, D. A., \& Goldman, M. S. (2003). Using a modified Stroop task to implicitly discern the cognitive organization of alcohol expectancies. Journal of Abnormal Psychology, 112, 171-175.

Krank, M., Wall, A. M., Stewart, S. H., Wiers, R. W., \& Goldman, M. S. (2005). Context effects on alcohol cognitions. Alcoholism: Clinical and Experimental Research, 29, 196-206.

Krystal, J. H., Cramer, J. A., Krol, W. F., Kirk, G. F., \& Rosenheck, R. A. (2001). Naltrexone in the treatment of alcohol dependence. New England Journal of Medicine, 345, 1734-1739.

Le Foll, B., \& Goldberg, S. R. (2005). Cannabinoid CB-sub-1 Receptor Antagonists as Promising New Medications for Drug Dependence. Journal of Pharmacology and Experimental Therapeutics, 312 , 875-883.

Lerman, C., Caporaso, N., Main, D., Audrain, J., Boyd, N. R., Bowman, E. D., \& Shields, P. G. (1998). Depression and self-medication with nicotine: the modifying influence of the dopamine D4 receptor gene. Health Psychology, 17, 56-62. 


\section{REFERENCES}

Lerman, C., Wileyto, E. P., Patterson, F., Rukstalis, M., Audrain McGovern, J., Restine, S., Shields, P. G., Kaufmann, V., Redden, D., Benowitz, N., \& Berrettini, W.H. (2004). The functional mu opioid receptor (OPRM1) Asn40Asp variant predicts short-term response to nicotine replacement therapy in a clinical trial. The Pharmacogenomics Journal, 4, 184-192.

Lichter, J. B., Barr, C. L., Kenedy, J. L., Van Tol, H. H. M., Kidd, K. K., \& Livak, K. J. (1993). A hypervariable segment in the human dopamine receptor D4 (DRD4). Human Molecular Genetics, 2, 767-773.

Logan, G. D. (2002). An instance theory of attention and memory. Psychological Review, 109, 376-400.

Love, A., James, D., \& Willner, P. (1998). A comparison of two alcohol craving questionnaires. Addiction, 93, 1091-1102.

MacLeod, C. M. (1991). Half a century of research on the Stroop effect: An integrative review. Psychological Bulletin, 109,163-203.

Maldonado, R., Valverde, O., \& Berrendero, F. (2006). Involvement of the endocannabinoid system in drug addiction. Trends in Neurosciences, 29, 225-232.

Mann, R. E., Sobell, L. C., Sobell, M. B., \& Pavan, D. (1985). Reliability of a family tree questionnaire for assessing family history of alcohol problems. Drug and Alcohol Dependence, 15, 61-67.

Marissen, A. E., Franken, I. H. A., Waters, A. J., Blanken, P., Van den Brink, W., \& Hendriks, V. M. (2006). Attentional bias predicts heroin relapse following treatment. Addiction, 101, 1306-1312.

Marlatt, G. A., \& Gordon, J. R. (Eds.) (1985). Relapse prevention: Maintenance strategies in the treatment of addictive behaviors. New York: Guilford Press.

Martin, C. S., Earleywine, M., Musty, R. E., Perrine, M. W., \& Swift, R. M. (1993). Development and validation of the Biphasic Alcohol Effects Scale. Alcoholism: Clinical and Experimental Research, 17, 140146.

McGeary, J. E., Monti, P. M., Rohsenow, D. J., Tidey, J., Swift, R., \& Miranda, J. (2005). Mu-opioid receptor gene polymorphism moderates naltrexone's effects on cue reactivity in non-treatment seeking heavy drinkers. Alcoholism: Clinical and Experimental Research, 29, 133A (Abstract).

McGue, M. (1999). Behavioral Genetic Models of Alcoholism and Drinking. In K. E. Leonard \& H. T. Blane (Eds.), Psychological theories of drinking and alcoholism (pp. 372-421). New York, NY, USA: The Guilford Press.

McLellan, A. T., Lewis, D. C., O'Brien, C. P., \& Kleber, H. D. (2000). Drug dependence, a chronic medical illness: implications for treatment, insurance, and outcomes evaluation. The journal of the American Medical Association, 284, 1689-95.

McNair, D. M., Lorr, M., \& Droppelman, L. F. (1971). Profile of Mood States (Manual). Educational and Industrial Testing Service, San Diego.

Modell, J. G., Mountz, J. M., Glaser, F. B., \& Lee, J. Y. (1993) Effect of haloperidol on measures of craving and impaired control in alcoholic subjects. Alcoholism: Clinical and Experimental Research, 17, 234-240.

Mogg, K., \& Bradley, B. P. (1998). A cognitive-motivational analysis of anxiety. Behaviour Research and Therapy, 36, 809-848.

Mogg, K., Bradley, B. P., Field, M., \& De Houwer, J. (2003). Eye movements to smoking-related pictures in smokers: Relationship between attentional biases and implicit and explicit measures of stimulus valence. Addiction, 98, 825-836.

Mogg, K., Field, M., \& Bradley, B. P. (2005). Attentional and approach biases for smoking cues in smokers: an investigation of competing theoretical views of addiction. Psychopharmacology, 180, 333-341.

Monterosso, J. R., Flannery, B. A., Pettinati, H. M., Oslin, D. W., Rukstalis, M., O'Brien, C. P., \& Volpicelli, J. R. (2001). Predicting treatment response to naltrexone: The influence of craving and family history. American Journal on Addictions, 10, 258-268. 
Monti, P. M., Binkoff, J. A., Abrams, D. B., Zwick, W. R., Nirenberg, T. D., \& Liepman, M. R. (1987). Reactivity of alcoholics and nonalcoholics to drinking cues. Journal of Abnormal Psychology, 96, 122 126.

Morren, M., Kindt, M., van den Hout, M., \& van Kasteren, H. (2003). Anxiety and the processing of threat in children: Further examination of the cognitive inhibition hypothesis. Behaviour Change, 20, 131 142.

Myrick, H., Anton, R. F., Li, X., Henderson, S., Drobes, D., Voronin, K., \& George, M. S. (2004). Differential brain activity in alcoholics and social drinkers to alcohol cues: relationship to craving. Neuropsychopharmacology, 29, 393-402.

Nederkoorn, C., \& Jansen, A. (2002). Cue reactivity and regulation of food intake. Eating Behaviors, 3, $61-72$.

Newlin, D. B., \& Thomson, J. B. (1990). Alcohol challenge with sons of alcoholics: A critical review and analysis. Psychological Bulletin, 108, 383-402.

Newlin, D. B., \& Thomson, J. B. (1991). Chronic tolerance and sensitization to alcohol in sons of alcoholics. Alcoholism: Clinical and Experimental Research, 15, 399-405.

NIAAA (2005). Alcohol and development in youth: A multidisciplinary overview. Alcohol Research and Health, 28.

O'Brien, C. P., Volpicelli, L. A., \& Volpicelli, J. R. (1996). Naltrexone in the treatment of alcoholism: A clinical review. Alcohol, 13, 35-39.

O'Malley, S. S., Jaffe, A. J., Rode, S., \& Rounsaville, B. J. (1996). Experience of a "slip" among alcoholics treated with naltrexone or placebo. American Journal of Psychiatry, 153, 281-283.

O'Malley, S. S., \& Maisto, S. A. (1985). Effects of family drinking history and expectancies on responses to alcohol in men. Journal of Studies on Alcohol, 46, 289-297.

Oslin, D. W., Berrettini, W., Kranzler, H. R., Pettinati, H., Gelernter, J., Volpicelli, J. R., \& O’Brien, C. P. (2003). A functional polymorphism of the mu-opioid receptor gene is associated with naltrexone response in alcohol-dependent patients. Neuropsychopharmacology, 28, 1546-1552.

Ostafin, B. D., \& Palfai, T. P. (2006). Compelled to consume: The Implicit Association Test and automatic alcohol motivation. Psychology of Addictive Behaviors, 20, 322-327.

Palfai, T., Davidson, D., \& Swift, R. (1999). Influence of naltrexone on cue-elicited craving among hazardous drinkers: The moderational role of positive outcome expectancies. Experimental and Clinical Psychopharmacology, 7, 266-273.

Palfai, T. P., \& Ostafin, B. D. (2003). Alcohol-related motivational tendencies in hazardous drinkers: Assessing implicit response tendencies using the modified-IAT. Behavior Research and Therapy, 41, $1149-1162$.

Peciña, S., \& Berridge, K. C. (2000). Opioid site in nucleus accumbens shell mediates eating and hedonic "liking' for food: Map based on microinjection Fos plumes. Brain Research, 863, 71-86.

Penke, L., Eichstaedt, J., \& Asendorpf, J. B. (in press). Single attribute Implicit Association Tests (SA-IAT) for the assessment of unipolar constructs: The case of sociosexuality. Experimental Psychology.

Peterson, J. B., Pihl, R. O., Gianoulakis, C., Conrod, P., Finn, P. R., Stewart, S. H., LeMarquand, D. G., \& Bruce, K. R. (1996). Ethanol-induced change in cardiac and endogenous opiate function and risk for alcoholism. Alcoholism: Clinical and Experimental Research, 20, 1542-1552.

Pettinati, H. M., Volpicelli, J. R., Pierce, J. D., \& O'-Brien, C. P. (2000). Improving naltrexone response: An intervention for medical practitioners to enhance medication compliance in alcohol dependent patients. Journal of Addictive Diseases, 19, 71-83.

Phelps, E. A., O'Connor, K. J., Cunningham, W. A., Funayama, E., Gatenby, J., Gore, J. C., \& Banaji, M. R. (2000). Performance on indirect measures of race evaluation predicts amygdala activation. Journal of Cognitive Neuroscience, 12, 729-738. 


\section{REFERENCES}

Piazza, P. V., Maccari, S., Deminiere, J. M., Le Moal, M., Mormede, P., \& Simon, H. (1991). Corticosterone levels determine individual vulnerability to amphetamine self-administration. Proceedings of the National Academy of Sciences of the United States of America, 88, 2088-2092.

Pierucci-Lagha, A., Covault, J., Herman, A., \& Kranzler, H. R. (2006). The cannabinoid receptor gene (CNR1) moderates the subjective response to alcohol in healthy individuals. Alcoholism: Clinical and Experimental Research, 30-Suppl, 195A (abstract).

Rajan, I., Murthy, P. V., Ramakrishnan, A. G., Gangadhar, B. N., \& Janakiramaiah, N. (1998). Heart rate variability as an index of cue reactivity in alcoholics. Biological Psychiatry, 43, 544-546.

Rather, B. C., \& Goldman, M. S. (1994). Drinking-related differences in the memory organization of alcohol expectancies. Experimental and Clinical Psychopharmacology, 2, 167-183.

Rather, B. C., Goldman, M. S., Roehrich, L., \& Brannick, M. (1992). Empirical modeling of an alcohol expectancy memory network using multidimensional scaling. Journal of Abnormal Psychology, 101, 174-183.

Ray, L. A., \& Hutchison, K. E. (2004). A polymorphism of the mu-opioid receptor gene (OPRMT) and sensitivity to the effects of alcohol in humans. Alcoholism: Clinical and Experimantal Research, 28, 1789-1795.

Robins, L. N., Wing, J., Wittchen, H. U., Helzer, J. E., Babor, T. F., Burke, J., Farmer, A., Jablensky, A., Pickens, R., Regier, D.A., Sartorius, N., \& Towle, L.H. (1989). The Composite International Diagnostic Interview: An epidemiologic instrument suitable for use in conjunction with different diagnostic systems and in different cultures. Archives of General Psychiatry, 45, 1069-1077.

Robinson, M. D., Meier, B. P., Zetocha, K. J., \& McCaul, K. D. (2005). Smoking and the Implicit Association Test: When the Contrast Category Determines the Theoretical Conclusions. Basic and Applied Social Psychology, 27, 201-212.

Robinson, T. E., \& Berridge, K. C. (1993). The neural basis of drug craving: An incentive-sensitization theory of addiction. Brain Research Reviews, 18, 247-291.

Robinson, T. E., \& Berridge, K. C. (2000). The psychology and neurobiology of addiction: An incentivesensitization view. Addiction, 95 (Suppl), $91-$ S117.

Robinson, T. E., \& Berridge, K. C. (2001). Incentive-sensitization and addiction. Addiction, 96, 103-114.

Robinson, T. E., \& Berridge, K. C. (2003). Addiction. Annual Review of Psychology, 54, 25-53.

Rohsenow, D. J., Monti, P. M., Hutchison, K. E., Swift, R. M., Colby, S. M., \& Kaplan, G. B. (2000). Naltrexone's effects on reactivity to alcohol cues among alcoholic men. Journal of Abnormal Psychology, 109, 738-742.

Rohsenow, D. J., Monti, P. M., Rubonis, A. V., Sirota, A. D., Niaura, R. S., Colby, S. M., Munroe Wunschel, S., \& Abrams, D. B. (1994). Cue reactivity as a predictor of drinking among male alcoholics. Journal of Consulting and Clinical Psychology, 62, 620-626.

Roozen, H. G., de Waart, R., van der Windt, D. A., van den Brink, W., de Jong, C. A., \& Kerkhof, A. J. (2006). A systematic review of the effectiveness of naltrexone in the maintenance treatment of opioid and alcohol dependence. European Neuropsychopharmacology, 16, 31 1-23.

Russell, J. A., Weiss, A., \& Mendelsohn, G. A. (1989). Affect Grid: A single-item scale of pleasure and arousal. Journal of Personality and Social Psychology, 57, 493-502.

Ryan, F. (2002). AB and alcohol dependence: A controlled study using the modified Stroop paradigm. Addictive Behaviors, 27, 471-482.

Sander, T., Harms, H., Dufeu, P., Kuhn, S., Rommelspacher, H., Schmidt, L. G. (1997). Dopamine D4 receptor Exon III alleles and variation of novelty seeking in alcoholics. American Journal of Medical Genetics, 74, 483-487.

Saunders, J. B., Aasland, O. G., Babor, T. F., de la Fuente, J. R., \& Grant, M. (1993). Development of the Alcohol Use Disorders Identification Test (AUDIT): WHO collaborative project on early detection of persons with harmful alcohol consumption: II. Addiction, 88, 791-804. 
Schinka, J. A., Busch, R. M., \& Robichaux Keene, N. (2004). A meta-analysis of the association between the serotonin transporter gene polymorphism (5-HTTLPR) and trait anxiety. Molecular Psychiatry, 9, 197-202.

Schippers, G. M., De Jong, C. A. J., Lehert, Ph., Potgieter, A., Deckers, F., Casselman, J., \& Geerlings, P. J. (1997). The Obessive Compulsive Drinking Scale: translation into Dutch and possible modifications. European Addiction Research, 3, 116-122.

Schmidt, L. G., Samochowiec, J., Finckh, U., Fiszer Piosik, E., Horodnicki, J., Wendel, B., Rommelspacher, H., \& Hoehe, M. R. (2002). Association of a CB1 cannabinoid receptor gene (CNR1) polymorphism with severe alcohol dependence. Drug and Alcohol Dependence, 65, 221-224.

Schoots, O. \& Van Tol, H. H. (2003). The human dopamine D4 receptor repeat sequences modulate expression. The Pharmacogenomics Journal, 3, 343-348.

Schott, B. H., Henson, R. N., Richardson Klavehn, A., Becker, C., Thoma, V., Heinze, H. J., \& Duzel, E. (2005). Redefining implicit and explicit memory: the functional neuroanatomy of priming, remembering, and control of retrieval. Proceedings of the National Academy of Sciences of the United States of America, 102, 1257-1262.

Schuckit, M. A. (1985). Ethanol-induced changes in body sway in men at high alcoholism risk. Archives of General Psychiatry, 42, 375-379.

Schuckit, M. A. (1994). Low level of response to alcohol as a predictor of future alcoholism. American Journal of Psychiatry, 151, 184-189.

Schuckit, M. A., \& Smith, T. L. (1996). An 8-year follow-up of 450 sons of alcoholic and control subjects. Archives of General Psychiatry, 53, 202-210.

Schuckit, M. A., Danko, G. P., Smith, T. L., Hesselbrock, V., Kramer, J., \& Bucholz, K. (2003). A 5-year prospective evaluation of DSM-IV alcohol dependence with and without a physiological component. Alcoholism: Clinical and Experimental Research, 27, 818-825.

Schuckit, M. A., Gold, E., \& Risch, C. (1987a). Plasma cortisol levels following ethanol in sons of alcoholics and controls. Archives of General Psychiatry, 44, 942-945.

Schuckit, M. A., Gold, E., \& Risch, C. (1987b). Serum prolactin levels in sons of alcoholics and control subjects. The American Journal of Psychiatry, 144, 854-859.

Self, D. W. (1998). Neural substrates of drug craving and relapse in drug addiction. Annals of Medicine, 30, 379-389.

Shao, C., Li, Y., Jiang, K., Zhang, D., Xu, Y., Lin, L., Wang, Q., Zhao, M., \& Jin, L. (2006) Dopamine D4 receptor polymorphism modulates cue-elicited heroin craving in Chinese. Psychopharmacology, 186, 185-190.

Sharma, D., Albery, I. P., \& Cook, C. (2001). Selective attentional bias to alcohol related stimuli in problem drinkers and non-problem drinkers. Addiction, 96, 285-295.

Sher, K. J. (1991). Children of alcoholics: A critical appraisal of theory and research. University of Chicago Press, Chicago, IL, USA.

Sherman, S. J., Rose, J. S., Koch, K., Presson, C. C., \& Chassin, L. (2003). Implicit and explicit attitudes toward cigarette smoking: The effects of context and motivation. Journal of Social and Clinical Psychology, 22, 13-39.

Smolka, M., Sander, T., Schmidt, L. G., Samochowiec, J., Rommelspacher, H., Gscheidel, N., Wendel, B., \& Hoehe, M. R. (1999). Mu-opioid receptor variants and dopaminergic sensitivity in alcohol withdrawal. Psychoneuroendocrinology, 24, 629-638.

Sobell, L. C., \& Sobell, M. B. (1990). Self-report issues in alcohol abuse: State of the art and future directions. Behavioral Assessment, 12, 77-90.

Sobell, L. C., \& Sobell, M. B. (1992). Timeline follow-back: A technique for assessing self-reported alcohol consumption. In R. Z. Litten \& J. P. Allen (Eds.), Measuring alcohol consumption: Psychosocial and biochemical methods (pp. 41-72). Totowa, NJ: Humana Press Inc. 


\section{REFERENCES}

Squire, L. R., Knowlton, B., \& Musen, G. (1993). The structure and organization of memory. Annual Review of Psychology, 44, 453-495.

Stacy, A. W. (1997). Memory activation and expectancy as prospective predictors of alcohol and marijuana use. Journal of Abnormal Psychology, 106, 61-73.

Stacy, A. W., Newcomb, M. D., \& Bentler, P. M. (1991). Cognitive motivation and drug use: A 9-year longitudinal study. Journal of Abnormal Psychology, 100, 502-515.

Stoof, J. C., \& Kebabian, J. W. (1981). Opposing roles for D-1 and D-2 dopamine receptors in efflux of cyclic AMP from rat neostriatum. Nature, 294, 366-368.

Stormark, K. M., Laberg, J. C., Nordby, H., \& Hugdahl, K. (2000). Alcoholics' selective attention to alcohol stimuli: Automated processing? Journal of Studies on Alcohol, 61, 18-23.

Strack, F., \& Deutsch, R. (2004). Reflective and Impulsive Determinants of Social Behavior. Personality and Social Psychology Review, 8, 220-247.

Swanson, J. E., Rudman, L. A., \& Greenwald, A. G. (2001). Using the implicit association test to investigate attitude-behaviour consistency for stigmatised behaviour. Cognition and Emotion, 15, 207-230.

Thush, C., \& Wiers, R. W. (in press). Explicit and implicit alcohol-related cognitions and the prediction of current and future drinking in adolescents. Addictive Behaviors.

Tiffany, S. T. (1990). A cognitive model of drug urges and drug-use behavior: role of automatic and nonautomatic processes. Psychological Review, 97, 147-168.

Van Breukelen, G. J. P. (2006). ANCOVA versus change from baseline: more power in randomized studies, more bias in nonrandomized studies. Journal of Clinical Epidemiology, 19, 36-52.

Van den Wildenberg, E., Beckers, M., van Lambaart, F., Conrod, P. J. \& Wiers, R. W. (2006). Is the strength of implicit alcohol associations correlated with alcohol-induced heart-rate acceleration? Alcoholism: Clinical and Experimental Research, 30, 1336-1348.

Van den Wildenberg E., Wiers R. W., Dessers J., Janssen R. G. J. H., Lambrichs E. H., Smeets H. J. M., \& van Breukelen G. J. P. (2007). A Functional Polymorphism of the Mu-Opioid Receptor Gene (OPRM1) Influences Cue-Induced Craving for Alcohol in Male Heavy Drinkers. Alcoholism: Clinical and Experimental Research, 31, 1-10.

Van Dijck, D., \& Knibbe, R. A. (2005). De prevalentie van probleemdrinken in Nederland: Een algemeen bevolkingsonderzoek [The prevalence of problem drinking in the Netherlands: A general screening of the population]. Universiteit Maastricht, Maastricht, the Netherlands.

Van Tol, H. H., Bunzow, J. R., Guan, H. C., Sunahara, R. K., Seeman, P., Niznik, H. B., \& Civelli, O. (1991). Cloning of the gene for a human dopamine D4 receptor with high affinity for the antipsychotic clozapine. Nature, 350, 610-614.

Van Tol, H. H., Wu, C. M., Guan, H. C., Ohara, K., Bunzow, J. R., Civelli, O., Kennedy, J., Seeman, P., Niznik, H. B., \& Jovanovic, V. (1992). Multiple dopamine D4 receptor variants in the human population. Nature, 358, 149-152.

Volpicelli, J. R., Alterman, A. I., Hayashida, M., \& O'Brien, C. P. (1992). Naltrexone in the treatment of alcohol dependence. Archives of General Psychiatry, 49, 876-880.

Volpicelli, J. R., Clay, K. L., Watson, N. T., \& Volpicelli, L. A. (1995). Naltrexone and the treatment of alcohol dependence. Alcohol Health and Research World, 18, 272-278.

Volpicelli J. R., Pettinati H. M., McLellan A. T., \& O'Brien C. P. (2001). Combining medication and psychosocial treatments for addictions: The BRENDA approach. New York, NY, US: Guilford Press.

Volpicelli, J. R., Watson, N. T., King, A. C., Sherman, C. E., \& O'Brien, C. P. (1995). Effect of naltrexone on alcohol "high" in alcoholics. American Journal of Psychiatry, 152, 613-615.

Waters, A. J., Sayette, M. A., \& Wertz, J. M. (2003). Carry-over effects can modulate emotional Stroop effects. Cognition \& Emotion, 17, 501-509.

Waters, A. J., Shiffman, S., Sayette, M. A., Paty, J. A., Gwaltney, C. J., \& Balabanis, M. H. (2003). Attentional bias predicts outcome in smoking cessation. Health Psychology, 22, 378-387. 
White, K. D. (1977). Salivation: A review and experimental investigation of major techniques. Psychophysiology, 14, 831-836.

White, H. R., \& Labouvie, E. W. (1989). Towards the assessment of adolescent problem drinking. Journal of Studies on Alcohol, 50, 30-37.

White, H. R., \& Labouvie, E. W. (2000). Longitudinal trends in problem drinking as measured by the Rutgers Alcohol Problem Index. Alcoholism: Clinical and Experimental Research, 24, 76A (Abstract).

Wiers, R. W. (2004). Wat is verslaving? De noodzaak van een conceptuele herdefiniering van het begrip verslaving. Gedrag \& Gezondheid, 32, 164-174.

Wiers, R. W. (in press a). Alcohol and drug expectancies as anticipated changes in affect: Negative reinforcement is not sedation. Substance Use \& Misuse.

Wiers, R. W. (in press b). Automatic and controlled psychological mechanisms in the enhanced risk for addiction in children of alcoholics and implications for interventions. In R. Velleman \& L. Templeton (Eds.), Coping with parental drinking: A European perspective. Oxford, UK: Oxford University Press.

Wiers, R. W., \& Stacy, A. W. (2006). Handbook of Implicit Cognition and Addiction. Thousand Oaks, CA: SAGE.

Wiers, R. W., \& Stacy, A. W. (2006). Implicit Cognition and Addiction: An Introduction. In R. W. Wiers \& A. W. Stacy (Eds.), Handbook of implicit cognition and addiction. Thousand Oaks, CA, US: Sage Publications.

Wiers, R. W., Gunning, W. B., \& Sergeant, J. A. (1998). Do young children of alcoholics hold more positive or negative alcohol-related expectancies then controls? Alcoholism: Clinical and Experimental Research, 22, 1855-1863.

Wiers, R. W., Hartgers, C., van den Brink, W., Gunning, W. B., \& Sergeant, J. A. (2000). A confirmatory analysis of the hierarchical structure of positive and negative dose-related alcohol expectancies in alcoholics and the associations with family history of alcoholism. Journal of Studies on Alcohol, 61, 177 186.

Wiers, R. W., Hoogeveen, K. J., Sergeant, J. A., \& Gunning, W. B. (1997). High- and low-dose alcoholrelated expectancies and the differential associations with drinking in male and female adolescents and young adults. Addiction, 92, 871-888.

Wiers, R. W., Houben, K., \& de Kraker, J. (2006). Implicit cocaine associations in active cocaine users and controls. Addictive Behaviors, 32, 1284-1289.

Wiers, R. W., van de Luitgaarden, J., van den Wildenberg, E., \& Smulders, F. T. Y. (2005). Challenging implicit and explicit alcohol-related cognitions in young heavy drinkers. Addiction, 100, 806-819.

Wiers, R. W., van Woerden, N., Smulders, F. T. Y., \& de Jong, P. J. (2002). Implicit and explicit alcoholrelated cognitions in heavy and light drinkers. Journal of Abnormal Psychology, 1 11 , 648-658.

Wigboldus, D. H. J., Holland, R. W., \& van Knippenberg, A. (2006). Single target implicit associations. Unpublished Manuscript.

Williams, J. G., Mathews, A., \& MacLeod, C. (1996). The emotional Stroop task and psychopathology. Psychological Bulletin, 120, 3-24.

Wilson, T. D., Lindsey, S., \& Schooler, T. Y. (2000). A model of dual attitudes. Psychological Review, 107, $101-126$.

Wise, R. A., \& Bozarth, M. A. (1987). A psychomotor stimulant theory of addiction. Psychological Review, 94, 469-492.

Yoon, H. H., lacono, W. G., Malone, S. M., \& McGue, M. (2006). Using the brain P300 response to identify novel phenotypes reflecting genetic vulnerability for adolescent substance misuse. Addictive Behaviors, 31, 1067-1087.

Zack, M., Stewart, S. H., Klein, R. M., Loba, P., \& Fragopoulos, F. (2005). Contingent gambling-drinking patterns and problem drinking severity moderate implicit gambling-alcohol associations in problem gamblers. Journal of Gambling Studies, 21, 325-354. 


\section{REFERENCES}

Zhang, P. W., Ishiguro, H., Ohtsuki, T., Hess, J., Carillo, F., Walther, D., Onaivi, E. S., Arinami, T., \& Uhl, G. R. (2004). Human cannabinoid receptor 1: 5' exons, candidate regulatory regions, polymorphisms, haplotypes and association with polysubstance abuse. Molecular Psychiatry, 9, 916-931.

Zhang, Y., Wang, D., Johnson, A. D., Papp, A. C., \& Sadee, W. (2005). Allelic expression imbalance of human mu opioid receptor (OPRM1) caused by variant A1 18G. The Journal of Biological Chemistry, 280, 32618-32624. 


\section{SUMMARY}

In this thesis several studies are described that explore cognitive and biological factors that could be related to the vulnerability for developing alcohol-related problems. Not everybody is equally at risk for developing an alcohol-related disorder. In individuals suffering from alcohol dependence, one can say that more or less half or $50 \%$ of the disease is determined or influenced by genetic factors. Alcoholism is a polygenetic disease which means that multiple genes are involved that all add to the development of an alcohol problem. Obviously, alcoholism is also influenced by environmental factors such as parents, peers, the media and the overall environment where one is brought up (e.g. country, culture, religion). Genes (the genotype) interact with environmental factors. This determines the final phenotype (what you see). Thus, carrying 'all the wrong genes' in an environment where alcohol consumption is forbidden or not common will probably not lead to the phenotype alcoholism. However, carrying few genes predisposing to problematic alcohol use, in an environment where consumption of alcohol is very common (e.g. Russia or the aborigines) will increase this risk. The more genes you have that contribute to the liability for alcoholism in an environment where drinking alcohol is very common, the higher the risk of developing an alcohol-related problem.

After continued alcohol use, structural changes occur in the brain, or more specifically, in the reward centre of the brain. The brain reward centre consists of several structures (e.g. the nucleus accumbens and ventral tegmental area) that reside deep in the brain. When a person uses alcohol or drugs, endogenous peptides or chemicals called beta-endorphins are released (amongst others). Beta-endorphins induce the release of a neurotransmitter called dopamine in areas such as the nucleus accumbens. Dopamine release is experienced as rewarding or reinforcing and it is this response that changes after prolonged alcohol (or drug) use. After repeated use, the release of dopamine immediately after administration of the drug (thus, during the rising blood alcohol curve in the case of alcohol) becomes stronger or 'sensitized'. This process is called incentive sensitization. It is the opposite of tolerance (where the effect of the drug becomes smaller), which also occurs, but in a later stage after administration, namely when blood alcohol levels are declining again. Thus, after repeated use one experiences a faster and steeper initial 'peak' of the alcohol or drug high (sensitization) which, however, also disappears faster (tolerance). Not everybody will be equally sensitive to the development of incentive sensitization where the drug cue obtains an incentive salience and can, sometimes without conscious awareness, guide behaviour towards drug use (attentional bias). Sensitization of the reward centre explains why an addicted person experiences a strong "wanting" of the drug, is compulsively looking for it and seems to have no control over his or her usage, while the "liking" of the drug has already disappeared. 
The goal of this thesis was to shed more light on different cognitive and biological variables on which individuals can differ in relation to their level of alcohol use and problems. Several biological individual differences in the response to alcohol use or exposure to alcohol cues were investigated such as (psycho)physiological indices (e.g. salivary reactivity and alcohol-induced heart-rate change) and genetic aspects (such as variations in the OPRM1, DRD4 and CB1 receptor genes). Genetic variations (polymorphisms) can moderate the response to alcohol and drugs, for example by altering receptor function, receptor expression and drug metabolism. The biological response to drug use is expected to be reflected on a cognitive level as well. Therefore, the biological aspects of interest were linked to several cognitive aspects to investigate the possible association between the different measures. Examples of cognitive factors that were investigated are explicit alcohol expectancies (the effect you expect alcohol to have on you, reported by means of a questionnaire), implicit associations with alcohol (reaction time tasks that measure the speed with which certain alcohol-related concepts are categorized. This is a measure of the automatic association someone has between 2 concepts, e.g. 'alcohol' and 'arousal'), cue-elicited craving (the urge to drink after exposure to an alcoholic beverage) and subjective arousal (how active you feel). Below, a summary of the main findings obtained in this thesis is provided.

Chapter 2 is focused on the relationship between several implicit measures and heart-rate acceleration after the administration of a relatively high dose of alcohol in a short period of time (based on body weight, more or less 5 shots of vodka in 5 minutes). More specifically, we first assessed implicit alcohol arousal and approach associations (measured with the Implicit Association Test or IAT) and attentional bias for alcohol-related stimuli (measured with an emotional Stroop task) in 48 male heavy drinkers. After performance of the implicit measures the high dose of alcohol was administered. Next, the heart-rate was assessed during 2 hours. The three types of implicit alcoholrelated cognitions that were assessed have all been proposed to reflect the strength of incentive sensitization that is experienced after repeated alcohol use. Thus, it was expected that heavy drinkers show strong associations (fast reaction times) between alcohol and arousal words and between alcohol and approach words on the IAT and more attentional distraction by alcohol words (attentional bias) on the Stroop. The strength of the arousal and approach associations and attentional bias was expected to be positively correlated with alcohol use and problems. Thus, the stronger the association between alcohol, arousal and approach words and the more a person is distracted by alcohol-related stimuli, the higher alcohol consumption and alcohol-related problems were expected to be. Furthermore, heart-rate (HR) acceleration during the ascending limb of the blood alcohol curve has proven to be a reliable measure of the sensitivity to the activating effects of alcohol. Individuals with a positive family history of alcoholism (FHP) have been found to show a stronger HR increase after alcohol consumption than 
FHNs, who do not have family members with an alcohol- problem. Therefore, we were interested if a positive relationship between the implicit measures and alcohol-induced heart-rate acceleration would exist. If this would be the case, it could suggest that the biological response to alcohol reflected by HR increase is translated into stronger implicit or automatic associations with alcohol and e.g. arousal on the IAT. Contrary to our hypothesis, none of the three implicit measures, nor alcohol-induced HR change was significantly related to alcohol use or problems. Furthermore, a negative association was found between implicit arousal-associations and alcohol-induced HR acceleration. This indicates that strong arousal-associations were correlated with a decrease in alcohol-induced HR. Approach-associations and attentional bias were not correlated with alcohol-induced HR acceleration, but both correlated positively with each other. Finally, a significant correlation was found between explicit alcohol arousal expectancies (measured before alcohol administration with a questionnaire) and the latency with which the peak in blood alcohol concentration (BAC) was reached. The stronger the explicit arousal expectancies, the faster blood alcohol concentrations went up. From these findings it can be concluded that alcohol-arousal associations and other implicit cognitions (attentional bias, approach associations) are not positively related to individual differences in the sensitivity to alcohol's activating effects, at least, not in the present sample consisting primarily of family history negative heavy drinkers. However, other research provides evidence which relates these implicit measures to anticipatory arousal, prior to alcohol intake. Possibly, in FHN drinkers the speed with which the peak $B A C$ is reached is a better measure of the activating effects someone experiences from alcohol than heart-rate acceleration.

Chapter 3 is focused on the relationship between the three implicit measures described above, in alcohol-dependent patients. Sixty-seven alcohol-dependent patients performed the arousal-IAT, the approach-avoidance IAT and the emotional Stroop, in a balanced order. It was hypothesized that the three measures would show a positive association because all have been hypothesized to reflect a sensitized appetitive response. Furthermore, individuals with FHP were expected to show stronger explicit arousal expectancies and implicit associations with arousal and approach than FHNs, due to their genetic predisposition and therefore stronger response to the rewarding effects of alcohol. In addition, a control group, consisting of 21 addiction clinic employees, was tested, to investigate whether the implicit cognitions would be specific for alcoholdependent patients. Results revealed that the three implicit measures were not significantly correlated in the patients. Unexpectedly, the groups did not differ on the unipolar arousal-IAT (contrast category neutral). Only the bipolar approach-avoidance IAT could differentiate between the groups, with patients associating alcohol less with avoidance than controls. A significant attentional bias on the Stroop was found only in the patients with a relatively short-term alcohol problem (not for long-term patients or controls). 
Their blocked Stroop-effect correlated .40 with the arousal-IAT. Explicit measures, such as expectancies and craving, were found to be more strongly associated with alcohol measures than the implicit measures. No differences were found between patients with a FHP or FHN on any of the measures. Taken together, there is no strong evidence that individual differences in implicit arousal associations are related to the severity of the alcohol problem. No correlation was found between implicit arousal associations and alcohol use, problems, age of onset or duration of the alcohol problem. Also, patients and controls did not differ in their alcohol arousal associations. The bipolar approachavoidance IAT could differentiate between patients and controls, suggesting that patients were less avoidant towards alcohol than controls. Furthermore, the approachavoidance IAT correlated positively with alcohol problems. Thus, it seems that the (bipolar) approach-avoidance IAT more reliably reflects individual differences in problematic alcohol use. Finally, the attentional bias finding in the short-term alcoholic patients could be interpreted as support for the incentive habit theory of addiction rather than the incentive sensitization theory. The incentive sensitization theory would predict a stronger attentional bias for alcohol cues as an addiction develops. The incentive habit theory states that as an addiction develops, the role of habit becomes more important, thus attentional bias would be expected to decrease.

Chapter 4 is focused on the prediction of relapse in alcohol-dependent patients receiving the mu-opioid receptor antagonist naltrexone for 3 months. Treatment outcome (relapse or not) of 19 alcohol-dependent patients was predicted with several explicit measures (craving, expectancies, motivation), implicit measures (arousal-IAT, approach-avoidance IAT, emotional Stroop) and several moderating variables (e.g. $\mathrm{FH})$. It was hypothesized that FHPs would be more successful than FHNs at remaining abstinent because there is evidence that naltrexone works especially well in patients with a genetic predisposition. Naltrexone blocks the mu-opioid receptor, to which betaendorphin bind after alcohol use. The beta-endorphinergic response is hereditary and seems to be stronger in FHPs than FHNs. By blocking the mu-opioid receptor, their more 'sensitive' beta-endorphin response is blocked, causing alcohol to loose its rewarding and reinforcing effect. The results did not confirm our hypotheses. It was found that patients who relapsed tended to show stronger implicit alcohol avoidance associations on the IAT, were less distracted by alcohol-related words on the Stroop and were predominantly Family History Positives (FHPs) compared with the patients who remained abstinent. Craving at treatment admission did not predict relapse. Although the study suffered from recruitment difficulties (the reason we were forced to drop the placebo condition), even in this small sample differences were found between patients who relapsed and those who did not. Implicit measures may have an additional value in the prediction of relapse in alcohol-dependent patients. 
Chapter 5 is focused on the relationship between the A1 $18 \mathrm{G}$ polymorphism of the mu-opioid receptor gene (OPRM1) and cue-reactivity after exposure to an alcoholic beverage. The mu-opioid receptor gene (OPRM1) codes for the mu-opioid receptor which binds beta-endorphin. The A118G polymorphism in this gene affects betaendorphin binding such that the Asp40 variant ( $G$ allele) binds beta-endorphin three times more tightly than the more common Asn40 variant (A allele). It was hypothesized that $G$ allele carriers would respond with stronger cue reactivity than the individuals homozygous for the A allele. Based on previous research, it was expected that the $G$ allele participants would more often report a FHP for alcoholism. In addition, the $G$ allele carriers were expected to report a higher alcohol and drug use. A total of 109 male heavy drinkers took part in the cue reactivity paradigm where they were exposed to water and beer in 3-minute trials. Subjective craving, subjective arousal and salivary reactivity were assessed as measures of cue-reactivity. $G$ allele carriers $(n=24)$ reported significantly more craving for alcohol than the A allele participants $(n=84)$ as was indicated by the larger beverage effect (difference score of beer - water trial). No differences were found for subjective arousal and saliva. Unexpectedly, the groups did not differ in family history of alcoholism. Participants with the $G$ allele reported a significantly higher lifetime prevalence of drug use than participants homozygous for the $A$ allele. Taken together, the findings in this chapter suggest that a stronger urge to drink alcohol after exposure to an alcoholic beverage might contribute to a heightened risk for developing alcohol-related problems in individuals with a copy of the $G$ allele. $G$ allele carriers might have more difficulty resisting a drink when offered. Given the higher drug use scores, the $G$ allele might also predispose to drug use in general.

Chapter 6 also focuses on the relationship between genetic variation in individuals and cue-reactivity. A sub sample of 88 heavy drinkers of the cue-reactivity study described in Chapter 5 provided a second informed consent for additional genotyping on the variable number of tandem repeats (VNTR) of the dopamine D4 receptor gene (DRD4) and the rs2023239 polymorphism of the cannabinoid CB1 receptor gene (CNR1). Both the dopamine and the cannabinoid receptor systems play an important role in the experience of reward after alcohol or drug use. It was hypothesized that individuals carrying the long allele of the DRD4 VNTR ( $>7$ repeats) and the individuals carrying a copy of the $\mathrm{C}$ allele of the CNRT polymorphism would report more cue reactivity and more alcohol and drug use than the DRD4 short allele or CNR1 TT allele participants, respectively. The less common alleles (or minor alleles) of both polymorphisms have been associated with a differential response to alcohol after consumption compared with their more common (or major) allele combination. Analyses of the cue-reactivity data revealed no strong evidence for stronger cue reactivity (= beverage effect, beer water trial) in the DRD4 L and CNR1 C allele groups. The DRD4 VNTR polymorphism tended to moderate salivary reactivity such that DRD4 $\mathrm{L}$ participants showed a larger 
beverage effect than the DRD4 S participants. Unexpectedly, the DRD4 L participants reported, on average over both exposure trials, less craving for alcohol and more subjective arousal than the DRD4 S participants. An interaction with alcohol use showed that as weekly alcohol consumption increased, the CNRI C allele group tended to report more craving for alcohol during the alcohol exposure than the $T$ allele group. From these findings it should be concluded that the DRD4 and CNR 1 polymorphisms do not appear to strongly moderate cue reactivity after alcohol cue exposure, in male heavy drinkers. However, both polymorphisms remain interesting candidates for pharmacotherapeutic treatment.

Taken together, the findings in this thesis with regard to several cognitive and biological variables in relation to problematic alcohol use, suggest that implicit cognitions such as arousal and approach associations and attentional bias do not necessarily tap into the (same) mechanism of incentive sensitization. No strong and consistent associations were found between the three implicit measures, or between the implicit measures and alcohol-induced heart-rate increase, or between the implicit measures and alcohol measures such as alcohol use, problems and age of onset. In addition, few differences were found between FHP and FHN alcoholics or between alcoholic patients and healthy controls. Explicit measures such as alcohol expectancies and craving, rather than implicit measures were related to alcohol measures and were able to distinguish patients from controls. Furthermore, explicit arousal expectancies were related to the speed with which the peak in blood alcohol concentration was reached. Genes are of great influence in predisposing individuals to engage in alcohol and drug abuse. Heavy drinking $G$ allele carriers experienced stronger craving for alcohol after alcohol exposure than AA individuals. This finding might explain why naltrexone seems to be more effective in patients carrying a $G$ allele of the mu-opioid receptor gene (OPRM1); possibly craving is reduced especially in this subgroup of patients. In addition, a copy of the $G$ allele of the A1 $18 \mathrm{G}$ polymorphism might render an individual sensitive to experiment more often with drugs in general. This could be inferred from the higher drug use scores that were found in this particular group. It should be noted that genes may interact with other genes as well as with environmental factors, thus presence of this polymorphism does not lead to the phenotype of alcohol or drug dependence through one final common pathway. The identification of genes that are involved in substance use disorders will enhance our understanding of addictive behaviors, such that new medications can be developed and prevention and treatment can be targeted more effectively. 


\section{SAMENVATTING}

In dit proefschrift worden enkele studies beschreven die cognitieve en biologische factoren onderzoeken die gerelateerd zouden kunnen zijn aan de gevoeligheid voor het ontwikkelen van een alcoholprobleem. Niet iedereen loopt namelijk een even groot risico om een alcoholprobleem te ontwikkelen. Je kunt zeggen dat in een individu dat lijdt aan alcohol afhankelijkheid ongeveer $50 \%$ van deze ziekte is bepaald of beïnvloed door genetische factoren. Alcoholisme is een polygenetische stoornis. Dat wil zeggen dat er meerdere genen betrokken zijn die bijdragen aan de ontwikkeling van een alcoholprobleem. Natuurlijk wordt alcoholisme ook door omgevings-factoren beïnvloed, zoals ouders, vrienden, de media en de algehele omgeving waarin iemand opgroeit (zoals land, cultuur, religie). Genen (het genotype) interacteren met omgevingsfactoren. Dit bepaalt het viteindelijke fenotype (wat je ziet). Dus, als je 'alle verkeerde genen' draagt, in een omgeving waar het drinken van alcohol verboden is, of niet gebruikelijk, dan zal dat waarschijnlijk niet leiden tot het fenotype alcoholisme. Als je weinig genen hebt die predisponeren tot alcohol misbruik, in een omgeving waar alcoholconsumptie heel gebruikelijk is (zoals in Rusland of bij de Aboriginals of in een studentenvereniging) dan loop je door je genen minder risico dan iemand anders in dezelfde omgeving, maar ben je nog niet immuun voor alcoholisme. Hoe meer genen je hebt die bijdragen aan gevoeligheid voor alcoholisme in een omgeving waar veel alcohol gedronken wordt, hoe groter je risico wordt.

$\mathrm{Na}$ aanhoudend alcoholgebruik treden er structurele veranderingen op in het brein, of meer specifiek, in het zogenaamde beloningssysteem van het brein. Dit beloningssysteem bestaat uit meerdere structuren, zoals de nucleus accumbens en het ventraal tegmentum, die zich diep in het brein bevinden. Als iemand alcohol of drugs gebruikt, komen er in dit systeem (onder andere) lichaamseigen eiwitten vrij; chemische stofjes die beta-endorfinen worden genoemd. Beta-endorfinen binden aan mu-opioïde receptoren en zorgen ervoor dat de neurotransmitter dopamine vrijkomt in gebieden zoals de nucleus accumbens. De afgifte van dopamine wordt als belonend of bekrachtigend ervaren en het is deze respons die verandert na herhaaldelijk gebruik van alcohol (of andere drugs). Naarmate er vaker gebruikt wordt, zal deze respons steeds sterker worden. Direct na inname van de drug (dus tijdens de stijgende bloed alcohol curve in het geval van alcohol) komt er meer dopamine vrij dan vroeger bij deze persoon het geval was, tijdens de beginfase van het alcoholgebruik. De reactie is 'gesensitiseerd' geraakt, oftewel gevoeliger geworden. Het resultaat van deze aanpassing in de hersenen wordt 'incentive sensitization' genoemd, dus overgevoeligheid voor beloning. Sensitisatie is het tegenovergestelde van tolerantie (waar het effect van de drug juist kleiner wordt). Tolerantie treedt ook op, maar tijdens een later stadium na toediening van de 
drug, namelijk wanneer de bloed alcohol concentratie weer daalt. De processen sensitisatie en tolerantie zijn dus tegengesteld aan elkaar maar treden beide op na herhaald druggebruik. De initiële reactie na alcohol- of druggebruik is een steeds snellere, hogere piek van de drug-high (sensitisatie), maar deze verdwijnt ook steeds sneller (tolerantie), waardoor opnieuw gebruikt moet worden om weer hetzelfde effect te ervaren. Niet iedereen is even gevoelig voor de ontwikkeling van deze beloningsovergevoeligheid waar de drug stimulus een zogenaamde 'incentive salience' verkrijgt, wat wil zeggen dat deze extra opvalt of in het oog springt. Drug-gerelateerde stimuli (oftewel cues) trekken de aandacht (aandachtsbias) en kunnen, soms zonder bewuste gewaarwording, het gedrag sturen. Robinson en Berridge onderscheiden subjectieve "wanting" en "liking" (tussen aanhalingstekens, want subjectief) en de neurale processen erachter, die niet subjectief hoeven te worden. Sensitisatie van het belongingssysteem verklaart waarom een verslaafde een hele sterke drang kan envaren om te gebruiken ("wanting"), er compulsief naar op zoek gaat en weinig controle heeft over zijn of haar gebruik, terwijl het plezierige effect ("liking") ervan op den duur verdwijnt.

Het doel van dit proefschrift is om meer licht te werpen op enkele verschillende cognitieve en biologische variabelen waarop individuen kunnen verschillen, in relatie tot hun niveau van alcoholgebruik en problemen. Verschillende cognitieve en biologische individuele verschillen in de respons op alcohol of na blootstelling aan alcohol stimuli zijn onderzocht, zoals: (psycho)fysiologische indices (speekselproductie en alcoholgeïnduceerde hartslagversnelling) en genetische aspecten (variaties in de OPRM1, DRD4 en CB1 receptor genen). Genetische variaties (polymorfismen) kunnen de respons op alcohol en drugs modereren, bijvoorbeeld door receptor functie, receptorexpressie of drugmetabolisme te veranderen. Het is aannemelijk dat de biologische reactie op drugs ook in cognitieve aanpassingen gereflecteerd wordt, bijvoorbeeld in sterkere positieve verwachtingen van alcohol. Daarom werden in deze thesis de biologische aspecten gerelateerd aan cognitieve variabelen om de mogelijke associatie tussen beide te onderzoeken. Voorbeelden van cognitieve factoren in deze dissertatie zijn expliciete alcohol verwachtingen en impliciete associaties met alcohol. Verwachtingen worden meestal gemeten met een vragenlijst, impliciete associaties bijvoorbeeld met reactietijdtaken. Deze reactietijdtaken meten de snelheid waarmee bepaalde alcoholgerelateerde concepten op een computer worden gecategoriseerd. Dit is een maat van de automatische associatie die iemand in het geheugen heeft tussen twee concepten, zoals 'alcohol' en 'actief'. Reactietijdtaken hebben enkele voordelen op vragenlijsten waar mensen o.a. sociaal wenselijk op kunnen antwoorden. Andere cognitieve variabelen waar naar gekeken is, zijn cue-geïnduceerde craving (de drang om te drinken na blootstelling aan een alcoholisch drankje) en subjectieve arousal (hoe veel 'opwinding' je ervaart na het drinken van alcohol of na blootstelling eraan). Hieronder volgt per hoofdstuk een samenvatting van de hoofdbevindingen uit dit proefschrift. 
Hoofdstuk 2 richt zich op de relatie tussen enkele impliciete maten (reactietijdtaken) en hartslagversnelling na de inname van een hoge dosis alcohol in korte tijd (afhankelijk van lichaamsgewicht ongeveer 5 shots wodka in 5 minuten tijd). Eerst werden bij de 48 mannelijke zware drinkers die deelnamen twee impliciete maten afgenomen. Een van de twee taken was de Impliciete Associatie Test (oftewel IAT). Hiermee werden associaties gemeten die de deelnemers hadden tussen alcohol en opwinding (arousal associaties) en tussen alcohol en benaderen (approach associaties). Dit gebeurt door woorden te classificeren met behulp van twee responsknoppen op het toetsenbord. Eerder onderzoek laat zien dat zwaardere drinkers sterkere impliciete associaties hebben tussen 'alcohol' en 'opwinding' en tussen 'alcohol' en 'benaderen' dan lichte drinkers. Dus werd verwacht dat zware drinkers op de IAT sterke associaties (snelle reactietijden) zouden laten zien tussen alcoholwoorden (bv. 'bier') en opwindingswoorden (bv. 'spraakzaam' of 'energiek') en tussen alcoholwoorden en woorden die een neiging tot benaderen vitdrukken (bv.'griipen' of 'naderen'). De tweede taak die werd afgenomen was de emotionele Stroop taak. Hiermee wordt gemeten in hoeverre de deelnemers worden afgeleid door alcohol-gerelateerde woorden. Tijdens de Stroop taak moeten woorden die op het scherm verschijnen alleen geclassificeerd worden op de kleur waarin ze verschijnen (rood, groen, geel of blauw, dus 4 mogelijke responsknoppen). Het is bekend vit onderzoek dat mensen die meer drinken trager reageren op alcoholwoorden dan op neutrale woorden omdat het lezen van een alcoholwoord afleidt. Dit interfereert met het kleurbenoemen, wat leidt tot een langzamere reactie (bv. het woord 'bier' in de kleur rood, wordt trager geclassificeerd als zijnde 'rood' dan 'brug' in de kleur rood). De mate van afleiding door alcohol-gerelateerde woorden wordt ook wel aandachtsbias voor alcohol-gerelateerde woorden genoemd (reactietijd alcohol woorden minus reactietijd neutrale woorden). Na het uitvoeren van deze reactietijdtaken werd de hoge dosis alcohol toegediend. Vervolgens werd gedurende 2 uur onder meer de hartslag gemeten. Het achterliggende idee is dat de 3 typen impliciete maten die zijn afgenomen de mate van 'incentive sensitization' reflecteren die ontstaat na herhaaldelijk alcoholgebruik. Voorspeld werd dat de sterkte van deze opwindings- en benaderingsassociaties en de mate van aandachtsbias positief zouden samenhangen met alcoholgebruik en -problemen. Dus, hoe sterker de associaties op de IAT tussen alcohol en opwindingswoorden en tussen alcohol en benaderingswoorden en hoe meer iemand wordt afgeleid door alcoholwoorden op de Stroop taak, hoe hoger de consumptie en het aantal alcoholproblemen verwacht werd te zijn. Verder is uit onderzoek naar voren gekomen dat hartslagversnelling na alcoholinname tijdens de stijgende bloed alcohol curve, een betrouwbare maat is voor de gevoeligheid voor de activerende effecten van alcohol. Individuen met een positieve familiegeschiedenis voor alcoholisme (dus mensen met meerdere alcoholisten in de familie) laten een sterkere hartslagversnelling zien na alcoholconsumptie (m.n. na een hoge dosis in korte tijd) vergeleken 
met mensen die geen biologische familieleden hebben met een alcoholprobleem (bij wie genen die predisponeren tot alcoholisme dus een minder sterke rol zullen spelen). Daarom werd onderzocht of er een positief verband gevonden zou worden tussen de impliciete maten en alcohol-geïnduceerde hartslagversnelling. Als dit zo zou zijn, zou dit kunnen betekenen dat de biologische reactie op alcohol, gereflecteerd door een sterkere hartslagversnelling, zich op cognitief vlak vertaalt in sterkere impliciete associaties tussen bijvoorbeeld alcohol en opwindingswoorden in de IAT.

Geen van bovenstaande hypotheses kon worden bevestigd. Geen van de impliciete maten, noch alcohol-geïnduceerde hartslagversnelling was significant gerelateerd aan alcoholgebruik en -problemen. Verder werd zelfs een negatieve correlatie gevonden tussen impliciete opwindingsassociaties en alcohol-geïnduceerde hartslagversnelling. Dit wil zeggen dat sterke opwindingsassociaties samenhingen met een daling in hartslag na alcoholinname. Benaderings-associaties en aandachtsbias correleerden niet met hartslagverandering na alcoholinname, maar hingen wel positief met elkaar samen. Tenslotte werd een sigificante relatie gevonden tussen expliciete alcoholverwachtingen van opwinding (vooraf gemeten met een vragenlijst) en de snelheid waarmee de maximale bloed alcohol concentratie werd bereikt. Hoe sterker de expliciete opwindings-verwachtingen van alcohol waren, hoe sneller de bloed alcohol concentratie omhoog ging na inname. Uit deze resultaten kan worden geconcludeerd dat impliciete cognities zoals opwindings- en benaderings-associaties en aandachtsbias voor alcohol, niet positief samenhangen met individuele verschillen in de gevoeligheid voor de activerende effecten van alcohol (hartslag), tenminste, niet in deze steekproef van zware drinkers met hoofdzakelijk een negatieve familiegeschiedenis voor alcoholisme (dus geen familieleden met een alcoholprobleem). Ander onderzoek laat zien dat impliciete maten wellicht eerder gerelateerd zijn aan anticipatoire opwinding vóór de inname van alcohol (en dus niet erna, zoals hier is gemeten). Wellicht is in drinkers die geen alcoholproblematiek in de familie hebben, de snelheid waarmee de piek in bloed alcohol concentraties wordt bereikt, een betere maat voor de activerende effecten van alcohol die iemand ervaart dan hartslagversnelling.

Hoofdstuk 3 richt zich op de relatie tussen de 3 hierboven besproken impliciete maten bij alcohol-afhankeliike patiënten. Bij 67 alcohol-afhankelijke patiënten werd de opwindings-IAT, de benaderen-vermijden IAT en de emotionele Stroop afgenomen. De hypothese was dat de 3 maten een positieve samenhang zouden vertonen omdat van alle drie werd verwacht dat zij een overgevoelig geworden appetitieve respons zouden reflecteren ('incentive sensitization'). Verder werd verwacht dat patiënten met een positieve familiegeschiedenis voor alcoholisme sterkere expliciete opwindingsverwachtingen zouden hebben van alcohol, evenals sterkere impliciete opwindings- en benaderingsassociaties dan patiënten met een negatieve familiegeschiedenis, vanwege hun genetische predispositie en daardoor sterkere biologische respons (belonend effect) na alco- 
holinname. Tenslotte werd een controlegroep van 21 medewerkers van verslavingsklinieken getest om te onderzoeken of deze impliciete cognities specifiek zouden zijn voor alcohol-afhankelijke patiënten of niet.

De resultaten lieten zien dat de 3 impliciete maten in de patiëntengroep niet met elkaar samenhingen. Als patiënten vergeleken werden met de controles bleek, geheel onverwachts, dat beide groepen niet verschilden in de sterkte van impliciete alcohol opwindingsassociaties (beide IAT-effecten waren $138 \mathrm{~ms}$ ). Alleen de bipolaire benaderen-vermiiden IAT kon de groepen differentiëren. Beide groepen lieten vermijdingsassociaties zien (alcoholwoorden werden sneller geclassificeerd met vermijdingswoorden onder dezelfde responsknop dan met benaderingswoorden). Bii de patiënten waren deze vermijdingsassociaties minder sterk dan bij de controle-proefpersonen. Een significante aandachtsbias voor alcoholwoorden (tragere reactietijden op alcoholwoorden in de Strooptaak) werd alleen gevonden bij alcohol-afhankelijke patiënten met een relatief 'korte duur' van het alcoholprobleem (dus niet bij de controles of bij de patiënten die al veel langer aan alcoholproblemen leden). Het Stroop-effect van de korte duur patiënten correleerde .40 met de opwindings-IAT. Expliciete maten zoals alcoholverwachtingen en drang naar alcohol, die waren gemeten met een vragenlijst, correleerden sterker met de alcoholmaten (gebruik, problemen etc.) dan de impliciete maten. Op geen van de maten werd een significant verschil gevonden tussen patiënten met en zonder familiegeschiedenis voor alcoholisme. Samenvattend kan worden gezegd dat er geen sterk bewijs is gevonden dat individuele verschillen in impliciete opwindingsassociaties gerelateerd zijn aan de ernst van het alcoloholprobleem. Er werd geen correlatie gevonden tussen impliciete opwindingsassociaties en alcoholgebruik, -problemen, leeftiid waarop het alcoholprobleem begon of de duur van het alcoholprobleem. Ook lieten patiënten en controles geen verschil zien in impliciete opwindingsassociaties. De bipolaire benaderen-vermijden IAT kon de groepen wel van elkaar onderscheiden; beide groepen hadden vermijdingsassociaties met alcohol, maar die van de controles waren sterker. Ook correleerde de benaderen-vermijden IAT positief met alcoholproblemen (hoe meer alcoholproblemen, hoe meer benaderingsassociaties met alcohol). Het lijkt er dus op dat de (bipolaire) benaderen-vermijden IAT een betrouwbaardere maat is van de individuele verschillen in alcoholgebruik. Tenslotte zou de bevinding dat alleen 'korte termijn' patiënten een aandachtsbias lieten zien, geïnterpreteerd kunnen worden in het teken van de 'incentive habit' theorie van verslaving in plaats van de hierboven besproken 'incentive sensitization' theorie. De 'incentive sensitization' theorie zou naar mate de verslaving zich ontwikkelt een steeds sterkere aandachtsbias voor alcohol stimuli voorspellen omdat het brein overgevoelig is geraakt voor beloning, waardoor alcohol stimuli de aandacht trekken. Volgens deze theorie zouden de 'lange termijn' patiënten dus het meest moeten worden afgeleid door alcoholwoorden. De 'incentive habit' theorie daarentegen, benadrukt het belang van 'gewoontegedrag' en 
vaste schema's of patronen die ontstaan als een verslaving zich verder ontwikkelt. Binnen deze theorie wordt voorspeld dat de aandachtsbias voor alcoholwoorden eerst toeneemt (zoals bij de korte termijn patiënten zichtbaar is), en na verloop van tijd weer afneemt (door de verschuiving naar gewoontegedrag waardoor minder aandacht nodig is voor alcohol stimuli). De patiënten met een langduriger alcoholprobleem lieten inderdaad geen aandachtsbias voor alcoholwoorden zien. Deze bevinding past beter binnen de 'incentive habit' theorie.

Hoofdstuk 4 richt zich op het voorspellen van terugval bij alcohol-afhankelijke patiënten die 3 maanden lang behandeld werden met de mu-opioïde receptor antagonist naltrexon. De behandeluitkomst (terugval of niet) van 19 alcohol-athankeliike patienten werd voorspeld met behulp van verschillende expliciete maten (drang naar alcohol, alcoholverwachtingen, motivatie om te stoppen met drinken), impliciete maten (opwindings-IAT, benaderen-vermijden IAT, emotionele Stroop) en verschillende modererende variabelen (zoals familiegeschiedenis voor alcoholisme). De hypothese was dat patiënten met een positieve familiegeschiedenis voor alcoholisme succesvoller zouden zijn in abstinent blijven dan patiënten met een negatieve familiegeschiedenis. Achterliggende reden voor deze voorspelling was, dat onderzoek laat zien dat naltrexon met name goed werkt bij individiven met een sterke familiale lading van alcoholproblemen. Naltrexon blokkeert de mu-opioïde receptor, waaraan beta-enforfinen hechten die vrijkomen na alcoholgebruik. Deze beta-endorfinerge respons blijkt sterk erfelijk bepaald te zijn. Er is onderzoek dat laat zien dat deze reactie sterker is in individuen met een sterke familiale lading van alcoholproblemen vergeleken met individuen zonder alcoholisten in de familie. Door het blokkeren van de mu-opioïde receptor wordt het voor hun zo gevoelige systeem dus 'uitgeschakeld' waardoor alcohol zijn belonende effect verliest. Hierdoor zal de behoefte om alcohol te drinken minder worden, waardoor terugval minder snel optreedt of minder diep zal zijn dan wanneer deze receptoren niet geblokkeerd zouden zijn.

De resultaten lieten iets anders zien dan was verwacht. Patiënten die terugvielen waren juist met name individuen met een sterke familiale lading van alcoholproblematiek (6 van de 7 die terugvielen). Verder vertoonden de patiënten met een terugval minder aandachtsbias voor alcohol-gerelateerde woorden en neigden ze naar sterkere impliciete vermijdingsassociaties met alcohol dan de patiënten die wel abstinent waren gebleven. Drang naar alcohol aan het begin van de behandeling kon terugval niet voorspellen. Ondanks het feit dat de studie leed onder wervingsproblemen (waardoor de placebo conditie moest komen te vervallen), werden zelfs in deze kleine steekproef verschillen gevonden tussen patiënten die terugvielen en die abstinent bleven. Impliciete maten kunnen een toegevoegde waarde hebben in het voorspellen van terugval bij alcohol-afhankelijke patiënten. Familiegeschiedenis voor alcoholisme is wellicht een te groffe genetische maat voor het voorspellen van behandelsucces bij naltrexon. Varian- 
ten in het mu-opiö̈de receptor gen, dat voor de mu-opiö̈de receptor codeert, zijn wellicht betere voorspellers van de respons op naltrexon.

Hoofdstuk 5 richt zich op de relatie tussen een variant van het mu-opiö̈de receptor gen en cue-reactiviteit na blootstelling aan een alcoholisch drankie, in mannelijke zware drinkers. Cue-reactiviteit houdt in dat iemand wordt blootgesteld aan een cue (een stimulus) waarop een reactie plaatsvindt. In dit geval was de cue een glas water of een glas bier waaraan de proefpersoon werd blootgesteld. Reactiviteit werd gemeten met vragenlijsten (hoe groot is je drang naar alcohol? , hoeveel opwinding ervaar je nu?) en met een fysiologische maat, namelijk speekselproductie. Het mu-opioïde receptor gen (OPRM1) codeert voor de mu-opioïde receptor waaraan beta-endorfinen zich hechten (b.v. na alcohol- of druggebruik). Het A118G polymorfisme in dit gen is een zogenaamd 'single nucleotide polymorfisme' oftewel een mutatie waarbij een $A$ nucleotide in het DNA vervangen is door een $G$ nucleotide. Hierdoor wordt het aminozuur asparagine op positie 40 in de aminozuurketen vervangen door asparaginezuur, waardoor de mu-opiöde receptor anders is van opbouw. Dit is van invloed op de kracht waarmee beta-endorfinen aan de receptor hechten. Door de veranderde receptoropbouw, kan de Asp40 variant ( $G$ allel) beta-endorfinen 3 keer zo sterk binden als de meer voorkomende Asn40 variant (A allel). Alcohol heeft dus een sterker effect op dragers van het $G$ allel (dus combinatie $A / G$ of $G / G$ ). De hypothese was dat deze personen met een sterkere cue reactiviteit zouden reageren na blootstelling aan alcohol, dan de individuen die homozygoot waren voor het A allel (combinatie A/A). Gebaseerd op eerder onderzoek werd verder voorspeld dat $G$ allel proefpersonen vaker een positieve familiegeschiedenis voor alcoholisme zouden rapporteren evenals meer alcohol en druggebruik. In totaal namen 109 mannelijke zware drinkers deel aan een cue reactiviteits paradigma, waarin zij werden blootgesteld aan water en vervolgens aan bier in perioden van drie minuten. Subjectieve drang naar alcohol (hoe veel zin in alcohol heb je?), subjectieve opwinding (hoe actief voel je je?) en speekselproductie (m.b.v. watjes) werden gemeten als indices van cue-reactiviteit.

Proefpersonen met een $\mathrm{G}$ allel $(n=24)$ lieten een significant sterkere drang naar alcohol zien dan de A allel proefpersonen $(n=84)$, zoals bleek vit een grotere verschilscore tussen de bier minus water aanbieding (sterkere toename in drang naar alcohol in de $G$ allel proefpersonen tijdens de blootstelling aan bier). Er werden geen verschillen gevonden in de mate van ervaren opwinding of speekselproductie. Wel produceerden de zwaardere drinkers meer speeksel dan de lichtere drinkers bij de blootstelling aan bier. Tegen de verwachting in verschilden de groepen niet in familiegeschiedenis voor alcoholisme. Proefpersonen met een $G$ allel rapporteerden meer verschillende soorten drugs te hebben gebruikt ooit in hun leven, dan proefpersonen die homozygoot waren voor het $\mathrm{A}$ allel. Met name hun gebruik van cannabis en amfetaminen lag hoger. Samengevat suggereren de bevindingen uit dit hoofdstuk dat een sterkere drang 
om te drinken na blootstelling aan alcohol, in individuen met een $G$ allel zou kunnen leiden tot een verhoogd risico voor het ontwikkelen van een alcoholprobleem. $G$ allel dragers hebben wellicht meer moeite om een drankje af te slaan als het hen wordt aangeboden. Gezien de hogere druggebruik scores zou het $G$ allel zelfs kunnen predisponeren tot het experimenteren met meer drugs in het algemeen.

Hoofdstuk 6 richt zich ook op de relatie tussen cue-reactiviteit en genetische variatie tussen individuen. Een subgroep van 88 proefpersonen uit de cue-reactiviteitsstudie vit Hoofdstuk 5 heeft een tweede keer toestemming gegeven voor additionele genotypering van hun 'variable number of tandem repeats' (VNTR) van het dopamine D4 receptor gen (DRD4) en het rs2023239 polymorfisme van het cannabinoïde CB1 receptor gen (CNR1). Zowel het dopaminerge als het cannabinoïde receptor systeem speelt een belangrijke rol bij het ervaren van beloningsgevoelens na inname van alcohol of drugs. Voorspeld werd dat individuen met de lange variant van de DRD4 VNTR (> 7 repeats, oftewel DRD4 L ) meer cue-reactiviteit zouden vertonen en meer alcohol en druggebruik zouden rapporteren dan individuen met het korte DRD4 allel $(<7$ repeats, oftewel DRD4 S). En er werd voorspeld dat individuen met een kopie van het $C$ allel van het CNRT polymorfisme (dus $\mathrm{C} / \mathrm{T}$ of $\mathrm{C} / \mathrm{C}$ ) meer cue-reactiviteit zouden vertonen en meer alcohol en druggebruik zouden rapporteren dan individuen, homozygoot voor het CNRT T allel (T/T). De minder vaak voorkomende allelen (oftewel 'minor' allelen) van beide polymorfismen zijn geassocieerd met een andere reactie op alcohol na inname, vergeleken met de meer voorkomende (oftewel 'major') allelcombinatie.

Analyses lieten zien dat er geen sterk bewijs was voor meer cue-reactiviteit (verschilscore, bier minus water aanbieding) in de DRD4 L en CNR1 C allel groepen. Het DRD4 VNTR polymorfisme neigde ernaar (net niet significant) om de mate van speekselproductie te modereren. DRD4 L proefpersonen lieten een grotere verschilscore zien in speekselproductie tussen de bier- en wateraanbieding dan de DRD4 S proefpersonen. Tegen de verwachting in rapporteerden de DRD4 $\mathrm{L}$ proefpersonen gemiddeld genomen over beide blootstellingen (water en bier samen), minder drang naar alcohol en meer subjectieve opwinding dan de DRD4 S proefpersonen. Een borderline significante interactie met alcoholgebruik liet zien dat de drang naar alcohol in de $\mathrm{C}$ allel proefpersonen tijdens blootstelling aan alcohol sterker werd naarmate ze een hoger wekelijks alcoholgebruik hadden, vergeleken met de T allel proefpersonen. Uit deze bevindingen moet worden geconcludeerd dat de DRD4 en CNR1 polymorfismen geen sterke rol lijken te spelen bij de mate van cue-reactiviteit na de blootstelling aan alcohol, in mannelijke zware drinkers.

De bevindingen die beschreven staan in dit proefschrift hebben betrekking op verschillende cognitieve en biologische variabelen in relatie tot problematisch alcoholgebruik. Samengevat kan worden gesteld dat impliciete cognities zoals opwindings- en benaderingsassociaties en aandachtsbias voor alcohol niet noodzakelijkerwijs teruggrij- 
pen op (hetzelfde) onderliggende mechanisme van 'incentive sensitization' oftewel overgevoeligheid voor beloning, als gevolg van structurele hersenveranderingen. Er zijn geen sterke of consistente associaties gevonden tussen de drie impliciete maten onderling, noch tussen de impliciete maten en alcohol-geïnduceerde hartslagversnelling, noch tussen de impliciete maten en alcoholmaten zoals alcoholgebruik, alcoholproblemen en leeftijd waarop het alcoholprobleem ontstond. Verder werden er weinig tot geen verschillen gevonden tussen alcoholisten met en zonder familiale lading van alcoholproblemen of tussen patiënten en controles. Expliciete maten zoals alcoholverwachtingen en drang naar alcohol hingen daarentegen wel samen met alcoholmaten en waren in staat patiënten en controles van elkaar te onderscheiden. Verder hingen expliciete opwindingsverwachtingen van alcohol samen met de snelheid waarop de maximale bloed alcohol concentratie werd bereikt in zware drinkers met een negatieve familiegeschiedenis voor alcoholisme. Genen spelen een belangriike rol bij de gevoeligheid voor alcohol en druggebruik. Zware drinkers met een $G$ allel van het mu-opioïde receptor gen (OPRMI ) kregen meer zin in alcohol na blootstelling eraan dan A/A individuen. Deze bevinding zou kunnen verklaren dat naltrexon extra effectief lijkt te zijn bij alcohol-afhankelijke patiënten met een $G$ allel. Wellicht treedt juist in deze groep een extra sterke daling in drang naar alcohol op door blokkade van de mu-opioïde receptor. Het kan zelfs zo zijn dat een kopie van het $G$ allel iemand kwetsbaarder maakt om in het algemeen met drugs te experimenteren. De hogere druggebruik scores die in deze groep werden gevonden wijzen in die richting. Het moet wel worden opgemerkt dat genen ook met andere genen kunnen interacteren en met omgevingsfactoren. Dus, aanwezigheid van dit polymorfisme hoeft niet via één direct pad te leiden tot het fenotype alcoholisme. De identificatie van genen die betrokken zijn bij middelengebruik vergroot ons begrip van verslavingsgedrag. Op deze wijze kunnen nieuwe medicijnen worden ontwikkeld en kunnen preventie en behandeling effectiever en meer gericht worden ingezet. 


\section{DANKWOORD}

Eindelijk, na vijf jaar letterlijk bloed, zweet en tranen ligt het er dan toch: $m^{\prime} n$ eigen boekje! Het is geen geheim dat mijn project vrij moeizaam is verlopen. Door meerdere tegenslagen heb ik veel momenten gehad waarop ik dacht ermee te stoppen. Gelukkig is dit, onder meer dankzii de steun en hulp van heel veel mensen om me heen, niet gebeurd. Ik wil dus erg graag van de gelegenheid gebruik maken om al deze mensen hiervoor ontzettend te bedanken: zonder jullie was het me nooit gelukt!

Allereerst gaat mijn dank uit naar mijn beide promotoren, Reinout en Anita. Ondanks de tegenslagen bleven jullie in mij geloven en mij steunen in de 'strijd'. Reinout, ook al waren we het misschien 'zakelijk' gezien niet altijd met elkaar eens, ik ben blij dat we het op een constructieve manier samen tot een einde hebben gebracht. Je dacht goed met me mee en droeg altijd nieuwe ideeën aan als een bepaald pad niet leek te werken. Ik noemde het soms 'surrealistisch optimisme' (;), maar het mag gezegd worden dat het viteindelijk gelukkig toch is goedgekomen! Je verbaasde me altijd weer met de snelheid waarmee je stukken nakeek en de goede feedback waarmee ik weer vit de voeten kon. Dankjewel daarvoor! En natuurlijk voor die extra maanden verlenging... Anita, jou wil ik onder meer bedanken voor het doorhakken van de knoop om de klinische studie stop te zetten (-). Je inzichten waren scherp en accuraat en bovenal erg menselijk. Je zei altijd dat het vooral ging om 'leuk onderzoek doen'. Daarin heb je volledig gelijk!

Mijn vele mede AiO's van wie de meeste goede vrienden zijn geworden hebben soms heel wat met me moeten doorstaan op de werkvloer... Ik ben jullie erg dankbaar dat jullie me altijd zijn blijven steunen! En natuurlijk was het naast de moeilijke momenten gelukkig ook heel vaak ontzettend gezellig, zowel op de uni tiidens onze eeuwige lunches, als daarbuiten, met veel borrel- en stapavondjes, chillen in de Zondag en eten van de afhaal-Indonees (-). Speciale woorden gaan vit naar mijn paranimfen Jill en Saskia. Dank jullie wel voor jullie eerlijke vriendschap, optimisme en peptalks. Zonder jullie was het 'leven' op de UM lang niet zo leuk geweest! Mijn kamergenootje Carolien wil ik heel hartelijk bedanken voor de vele uren geduld met mij en de gezellige tijden, onder meer op de RSA alcoholcongressen. Praktijkonderzoek hebben we nu wel onder de knie denk ik... (Joris, ook mede dankzij jou (:)). Dirk, Ellen D., Els, GJ, Hans, Hugo, Jade, Jochen, Joke, Katrijn, Loes, Marijn, Neeltje, Roy en last but zeker not least Tim, jullie zijn geweldige collega's en vrienden. Ik hoop nog vaak van jullie gezelschap te mogen genieten ook al woon ik nu wat verder weg. Ook de overige collega's van EP, hartelijk dank voor de prettige werksfeer, gezellige keuken-bijeenkomsten en Thembi- 
borrels. IT mannen van de $5^{\mathrm{e}}$, bedankt voor jullie technische en overige support en de gezellige BBQs bii Slavante :).

Rob, mijn "Geneticaman", dankjewel voor al je hulp en inzet bij het genetische deel van het onderzoek en de 'mini-colleges' die je me met veel enthousiasme gaf, soms onder het genot van een wijntje of een PX (-). Mede dankzij jou werd onderzoek doen weer leuk! Gerard, jou wil ik bedanken voor al je statistische adviezen, je ongelofelijk snelle, vitgebreide (en soms ook frustrerende...) feedback en je innemende verhalen voorafgaand of na een statistisch consult. Zo zie je maar: zelfs statistici hebben humor $(-)$ !

Another word of thanks goes out to two other co-authors, Kent Hutchison and Patricia Conrod. Kent, thank you for a nice stay in Boulder, Colorado. Your help with part of the genetics data and insights into the paper were valuable to me. Trisha, you know how tough naltrexone studies can be (-). Thank you for your cooperation on the heart-rate paper.

Mijn stagiaires en student/onderzoeksassistenten Margreet Beckers, Marten van de Braak, Wannes Coolen, Joelle Dessers, Femke van Lambaart en Tim Satiin wil ik van harte bedanken voor het verzamelen van een deel van de soms moeizame data. Zonder jullie was het niet gelukt! De verslavingsklinieken van de Mondriaan Zorggroep en het Verslavingszorgcircuit Noord- en Midden Limburg hebben gedurende een paar jaar onderzoek aan hun instellingen mogelijk gemaakt. Hiervoor wil ik Toon Wijdeveld, Peet Geenen, Hetty Verhaegh, Wim Meulders en alle andere medewerkers van harte bedanken. En zonder de zware drinkers en alcoholafhankelijke patiënten die deelnamen had dit onderzoek nooit kunnen plaatsvinden, bedankt voor jullie bijdrage aan de wetenschap!

Een welgemeend woord van 'on-dank' gaat uit naar de heer Batèl vit Parijs die mijn samenwerkingsaversie met Frankrijk heeft gevoed en waarschijnlijk nog steeds de surrealistische hoop koestert dat de klinische studie daar ooit nog van start zal gaan. Parijs zelf wordt er gelukkig niet minder leuk door (-)!

Ook buiten het werk om mag ik mij gelukkig prijzen met veel lieve vriendjes en vriendinnetjes die mij de afgelopen jaren door dik en dun gesteund hebben en voor veel ontspanning, de nodige (schater)lachbuien en bijzondere weekenden :) hebben gezorgd. Vriendschappen maken het leven kleurrijk en waardevol. Mijn "Borreltijd" vriendinnetjes; Anne K., Anne O., Fran, Jeanne, Marije, Marike, Sach en Thes; jullie weten hoe speciaal jullie voor me zijn en hoe zeer ik altijd weer geniet van onze momenten samen; in Nederland, Spanje, Frankrijk of waar dan ook. Kaas en wijn smaken zelden zo lekker als met jullie :)! Mijn "Heugem" vriendje en vriendinnetjes; An, Heidi, Juul, Keetie, Lon en Jeroen; bedankt voor de geweldige tijd samen in één huis. De 'gang-bijeenkomsten' hebben zich inmiddels verplaatst over heel Nederland, maar ik ben blij dat jullie nog steeds deel uit maken van mijn 
leven en hoop dat dat nog lang zo zal zijn! Mijn "Vodafone" vriendje en vriendinnetjes; Barb, Door, Ju, (andere (-)) Lonneke en Dennis; ik vind het heel bijzonder dat we elkaar na onze Vodafone-bijbaantjes zijn blijven zien. Ik denk nog steeds in 'woonplaatscodes' bij het horen van plaatsnamen en ben blij dat er nog medestanders zijn die hetzelfde lot delen (-). Christel, Carla, mijn Tilburgse roots, wat kennen we elkaar al lang! Dank jullie wel voor deze 'eeuwige' vriendschappen. En sommige mensen zijn nu eenmaal niet in hokjes te plaatsen $(;)$ : Lenne, Erik jullie zijn geweldig! leder op zijn eigen manier, maar allebei in ieder geval met heerlijke (groffe) humor. Buiten deze 'kern' van langdurige vriendschappen zijn er nog talloze andere mensen die mijn leven 'leuk maken'. Jullie ook bedankt voor de mooie momenten! Dat er nog maar veel mogen volgen.

Een extra speciale blijk van waardering gaat natuurlijk uit naar mijn fantastische ouders en broertie! Conny en Henk, zonder jullie was ik nooit geworden tot de persoon die ik nu ben. Jullie zijn altijd achter me blijven staan en hebben me alle kansen van de wereld gegeven om iets moois van het leven te maken. Ik ben jullie hiervoor ontzettend dankbaar; jullie zijn schatten! Job, je blijt me aan het lachen maken met je scherpe humor en me verbazen met de snelheid waarmee je altijd weer dingen voor me kunt vinden op het internet die mij niet lukken ("download here" ()). Enschede en Utrecht is eigenlijk niet zo ver van elkaar, toch?

En dit is dan die laatste paragraaf waarin je altijd die extra bijzondere persoon bedankt $;$. Thesje, aan jou wil ik nog een speciaal woord van dank wijden. Onze vriendschap is door de jaren heen heel belangrijk voor me geworden. We hebben zo veel samen beleefd, zo ontzettend hard gelachen, en gehuild (ik dan meestal (:)), en zo veel besproken onder het genot van een rosé'tje en 'een groot glas kraanwater' in de Zondag. Het is logisch dat ik je mis nu je voor 2 jaar in Nigeria zit. De mensen daar mogen in hun handjes klappen dat ze je een tijdje kunnen 'lenen' :-)! Dankjewel voor alles! 


\section{CURRICULUM VITAE}

Esther van den Wildenberg werd geboren op 13 oktober 1977, in Oosterhout. Zii behaalde haar WWO-diploma in 1995 aan het St. Odulphus Lyceum in Tilburg. $\mathrm{Na}$ één jaar buitenlandervaring in Salamanca, Spanje, begon zij haar studie Psychologie aan de Universiteit van Maastricht. In 2002 studeerde zij cum laude af in de biologische psychologie met specialisaties in de neuropsychologie, ontwikkelingspsychologie en psychopathologie. Daarna is zij tot eind 2006 als promovenda werkzaam geweest bij het Departement Experimentele Psychologie van de Universiteit Maastricht. Momenteel werkt zij als marketing onderzoeker en beleidsmedewerker bij de Stichting Alcoholpreventie (STAP) in Utrecht.

Esther van den Wildenberg was born on October 13th 1977, in Oosterhout. In 1995, she graduated from secondary school, St. Odulphus Lyceum, in Tilburg. After one year of experience abroad in Salamanca, Spain, she started studying Psychology at Maastricht University. In 2002 she graduated cum laude in biological psychology with specializations in neuropsychology, developmental psychology and psychopathology. In the same year, she began her PhD research at the Department of Experimental Psychology at Maastricht University. She is currently working as marketing researcher and policy advisor at the National Foundation for Alcohol Prevention (STAP) in Utrecht. 


\section{PUBLICATIONS}

Van den Wildenberg, E., Beckers, M., van Lambaart, F., Conrod, P. J., \& Wiers, R. W. (2006). Is the strength of implicit alcohol associations correlated with alcohol-induced heart-rate acceleration? Alcoholism: Clinical and Experimental Research, 30, 1336-1348.

Van den Wildenberg, E., Coolen, W., Satijn, T., \& Wiers, R. W. (2005). Do implicit alcohol arousal associations correlate with attentional bias in alcoholic patients? Alcoholism: Clinical and Experimental Research, 29, Supplement 26A (Abstract).

Van den Wildenberg, E., Coolen, W., Wiideveld, A. G. M., Meulders, W., \& Wiers, R. W. (2007). Do implicit alcohol arousal and approach associations correlate with attentional bias in alcohol-dependent patients? Manuscript submitted for publication.

Van den Wildenberg, E., Dessers J., Janssen R. G. J. H., Lambrichs E. H., Smeets H. J. M., \& Wiers R. W. (2006). A functional polymorphism of the mu-opioid receptor gene (OPRM1) influences subjective craving for alcohol in a cue-reactivity paradigm. Alcoholism: Clinical and Experimental Research, 30, Supplement 10A (Abstract).

Van den Wildenberg, E., Janssen, R. G. J. H., Hutchison, K. E., van Breukelen, G. J. P., \& Wiers, R. W. (in press). Polymorphisms of the dopamine D4 receptor gene (DRD4 VNTR) and cannabinoid $C B 1$ receptor gene are not strongly related to cue reactivity after alcohol exposure. Addiction Biology.

Van den Wildenberg, E., \& Wiers, R. W. (2003). Conditioned place preference as a measure of implicit alcohol-motivation in humans. Alcoholism: Clinical and Experimental Research, 27, Supplement 102A (Abstract).

Van den Wildenberg, E., Wiers, R. W., Dessers, J., Janssen, R. G. J. H., Lambrichs, E. H., Smeets, H. J. M., \& van Breukelen, G. J. P. (2007). A functional polymorphism of the muopioid receptor gene (OPRM1) influences cue-induced craving for alcohol in male heavy drinkers. Alcoholism: Clinical and Experimental Research, 31, 1-10.

Van den Wildenberg, E., Wiers, R. W., van Lambaart, F., \& Beckers, M. (2004). Is the strength of implicit alcohol associations correlated with alcohol-induced heart-rate acceleration? Alcoholism: Clinical and Experimental Research, 28, Supplement 67A (Abstract).

Van der Zwaluw, C. S., van den Wildenberg, E., Wiers, R. W., Franke, B., Scholte, R. H. J., \& Engels, R. C. M. E. (2007). Functional polymorphisms of the mu-opioid receptor gene (OPRM1) and the implications for alcohol dependence in humans. Manuscript submitted for publication.

Wiers, R. W., Bartholow, B. D., van den Wildenberg, E., Thush, C., Engels, R. C. M. E., Sher, K. J., Grenard, J., Ames, S. L., \& Stacy, A. W. (2007). Automatic and controlled processes and the development of addictive behaviors in adolescents: a review and a model. Pharmacology, Biochemistry and Behavior, 86, 263-283.

Wiers, R. W., Rinck, M., Kordts, R. Dictus, M., Houben, K., van den Wildenberg, E., \& Strack, F. (2007). Train addictive impulses away! Assessing and re-training automatic tendencies to drink. Manuscript submitted for publication.

Wiers, R. W., van de Luitgaarden, J., van den Wildenberg, E., \& Smulders, F. T. Y. (2005). Challenging implicit and explicit alcohol-related cognitions in young heavy drinkers. Addiction, 100, 806-819. 